\title{
TREE-WATER INTERACTIONS AT VARYING SPATIOTEMPORAL SCALES IN A WATER LIMITED ENVIRONMENT
}

\author{
Jeisson Leonardo Reyes-Acosta
}




\section{PhD dissertation committee}

Chair

prof. dr. ir. A. Veldkamp University of Twente

Promotor

prof. dr. Z. Su University of Twente, ITC

Co-promotor

dr. ir. Maciek W. Lubczynski University of Twente, ITC

Members

prof. dr. Andrew K. Skidmore University of Twente, ITC

dr. ir. dr. Y.A. Hussin University of Twente, ITC

prof. dr. Kathy Steppe

Ghent University

prof. dr. L. Adrian Bruijnzeel Vrije Universiteit Amsterdam

dr. Teresa Soares David INIAV, Portugal

dr. Christiaan van der Tol University of Twente, ITC

ITC dissertation number 272

ITC, P.O. Box 217, 7500 AE Enschede, The Netherlands

ISBN: $\quad$ 978-90-365-3896-1

DOI: $\quad$ http://dx.doi.org/10.3990/1.9789036538961

Printed by: ITC printing department, Enschede, The Netherlands

(c) Jeisson Leonardo Reyes-Acosta, Enschede, The Netherlands

(c) Cover design by Oscar Javier Reyes Acosta

All rights reserved. No part of this publication may be reproduced without the prior written permission of the author.

- 1 - FACULTY OF GEO-INFORMATION SCIENCE AND EARTH OBSERVATION 


\title{
TREE-WATER INTERACTIONS AT VARYING SPATIOTEMPORAL SCALES IN A WATER LIMITED ENVIRONMENT
}

\author{
DIS SERTATION
}

\author{
to obtain \\ the degree of doctor at the University of Twente, \\ on the authority of the rector magnificus, \\ prof. dr. H. Brinksma, \\ on account of the decision of the graduation committee, \\ to be publicly defended \\ on Wednesday, June 3, 2015 at 16:45
}

by

Jeisson Leonardo Reyes-Acosta

born on August 03, 1981

in Bogotá, Colombia 
This dissertation is approved by:

prof. dr. Z. Su (promotor)

dr. ir. Maciek W. Lubczynski (co-promotor) 


\section{Acknowledgments}

I want to extend my gratitude to all the people that directly and/or indirectly supported all the stages of my $\mathrm{PhD}$ experience. To my supervisor, Dr. Ir. Maciek Lubczynski, for his patience, involvement, passion, vision and guidance during all these years. To Prof. Bob Su, for his active and timely support in my PhD project. To Dr. J. Martínez Fernández at the Universidad de Salamanca, and all the members of "Grupo de recursos hídricos" (specially Dr. Nilda Sánchez Martín), for hosting me in their lab for three consecutive summers, while providing valuable advice and insights on the Spanish environments. To Lucio and his family for letting me roam free in their land installing weird devices in their trees. To Jean Roy for sharing his knowledge and developments for my project. To Prof. Kathy Steppe and Maurits Vandegehuchte at the University of Gent, for opening the doors of your lab to me, and for engaging on my research ideas. To all my PhD colleages at ITC, especially for those in room 5-116, who became an extension of my family. To Alain Pascal Frances, Enrico Balugani, Guido Baroncini Turrichia, Chandra Prasad Ghimire, Christiaan van der Tol and Tanvir Hassan for all the great memories and experiences shared in our field expeditions and in our scientific discussions. To all the MSc. students that I had the fortune to advise. To all the members of staff at ITC, particularly the Students Affairs staff for their unconditional support.

I am especially grateful to my wife Cornelia Reyes and my families, both in Colombia and in Austria, whose support was essential in moments of doubts and confusion. To my brother Oscar and my sister in law Natalia for helping with the design of the cover and for surviving hours of harsh field conditions in the peak of the Spanish summer time. Finally, to my sister Carolina for her cheerful demeanour and positiveness. Without my families this project would not have been successful.

To the trees, the water, the land, and the Spanish sky, thanks for being my hosts and companions in those long working days in the forest. 


\section{Contents}

Acknowledgments

$\begin{array}{ll}\text { Contents } & \text { ii }\end{array}$

List of Figures vii

$\begin{array}{ll}\text { List of Tables } & \text { XV }\end{array}$

1 Tree-water interactions in water limited environments 1

1.1 Introduction . . . . . . . . . . . . . 2

1.1.1 The role of transpiration in ecohydrological systems 2

1.2 Scientific considerations . . . . . . . . . . . . . 5

1.2.1 Brief overview of sap flow measurements . . . . . 5 5

1.2.2 Methods to scale up sap flow measurements to tree transpiration at different spatial scales . . . . . . 6

1.2.3 Water balance, transpiration and naturally abundant isotopes for water sourcing . . . . . . . . 7

1.2.4 Tree water flow modelling and sourcing of water

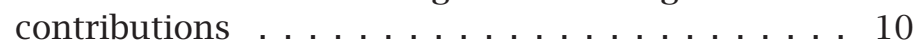

1.3 Problem statement and related novelty . . . . . . . . . 11

1.3.1 Natural temperature gradient bias on sap flow measurements . . . . . . . . . . . . . . 11

1.3.2 Optimisation of TDP sap flow measurements under semi-arid conditions . . . . . . . . . . . 12

1.3.3 Estimation of tree transpiration at different spatiotemporal scales using remotely sensed imagery . . 12

1.3.4 Sourcing tree transpiration by naturally abundant isotope tracing and tree water flow modelling . . . . 13

1.3.5 Unravelling the spatial variability of tree transpiration water sources $\ldots \ldots \ldots \ldots$

1.4 Research objectives . . . . . . . . . . . . . . . . . . . . 14

1.4 .1 Main objectives . . . . . . . . . . . . . . . 14

1.4 .2 Specific objectives . . . . . . . . . . . . . 14

1.5 Thesis outline $\ldots \ldots \ldots \ldots \ldots \ldots \ldots$ 
2 Correction of natural temperature gradients in sap flow measurements

2.1 Introduction . . . . . . . . . . . . . . . . . . 18

2.2 Materials and methods . . . . . . . . . . . . . 21

2.2.1 Collection and processing of plant material . . . . 21

2.2.2 Mariotte-based verification system . . . . . . . . . 22

2.2.3 Artificial thermal gradient enforcement . . . . . . . 23

2.2.4 Thermal dissipation probes for measuring sap flux densities . . . . . . . . . . . . . . . 24

2.2.5 Cyclic power scheme for correcting the influence of ATG in TDP . . . . . . . . . . . . . . 24

2.2 .6 Experimental design . . . . . . . . . . . . . . 26

2.2.7 Test 1: ATG formation monitoring . . . . . . . 26

2.2.8 Test 2: ATG effect upon the standard TDP signal and water flow estimations . . . . . . . . . 27

2.2.9 Test 3: Validation of the CHD method for NTG correction . . . . . . . . . . . . . . . . 29

2.2.10 Comparing CHD and TTD methods . . . . . . . . 30

2.2 .11 Error calculations . . . . . . . . . . . . . 31

2.3 Results and discussion . . . . . . . . . . . . . 32

2.3.1 Characteristics of the plant material . . . . . . . 32

2.3.2 Test 1: confirming ATG formation . . . . . . . 32

2.3.3 Test 2: understanding the biasing dynamics of ATGs 33

2.3.4 Test 3: assessing the accuracy of CHD to correct the ATG bias . . . . . . . . . . . . . . 35

2.3.5 Comparing cyclic power correction methods . . . . 39

2.3.6 Limitations of the CHD method for correcting ATGs 40

2.4 Conclusions . . . . . . . . . . . . . . . . . 43

3 Sap flow measurements optimisation under dry-conditions 45

3.1 Introduction . . . . . . . . . . . . . 46

3.2 Materials and methods . . . . . . . . . . . . . 48

3.2 .1 Study area . . . . . . . . . . . . . . 48

3.2.2 Monitoring climatic and hydrological variables . . . 48

3.2.3 Tree survey and stratified sampling for sap flow measurements . . . . . . . . . . . . . 49

3.2.4 Step-wise optimisation of TDP measurements . . . . 49

3.2.5 Step 1: NTG bias correction of TDP measurements by CHD method . . . . . . . . . . . . . 50

3.2.6 Step 2: Night flow $\Delta T_{\max }$ correction $\ldots \ldots \ldots \ldots 53$

3.2.7 Step 3: Radial and azimuthal TDP correction . . . . . 54

3.2.8 Criteria to assess the optimized TDP measurements 56

3.3 Results and Discussion . . . . . . . . . . . . . . . 56

3.3.1 Dry-season environmental conditions and selected days for comparison . . . . . . . . . . . . . . 56

3.3.2 Tree population structures of Q.i. and Q.p. . . . . 57

3.3.3 Thermal diffusivity of Q.i. and Q.p. . . . . . 57 
3.3.4 First step: Natural temperature gradients bias correction with the CHD method . . . . . . . . . 58

3.3.5 Second step: Night flow determination and correction 64

3.3.6 Third step: Accounting for radial-azimuthal sap flux density profiles in TDP measurements. . . . . . . . 65

3.4 Opportunities and final conclusions . . . . . . . . . 69

4 Dry-season tree transpiration in the Sardón catchment 77

4.1 Introduction . . . . . . . . . . . . 78

4.2 Materials and Methods . . . . . . . . . . . . 80

4.2 .1 Study Area . . . . . . . . . . . . . . . . . . . 81

4.2.2 Measurement of biometric characteristics . . . . . . 82

4.2.3 Sap flow measurements . . . . . . . . . . . . 83

4.2.4 Modelling of temporal sap-flow variability . . . . . 85

4.2.5 Remote sensing tree classification . . . . . . . 87

4.2.6 Accuracy assessment of the tree classification . . . 8 88

4.2.7 Scaling up sap flow measurements for transpiration mapping . . . . . . . . . . . . 90

4.2.8 Assessment of remote sensing scaling-up procedure 94

4.3 Results and Discussion . . . . . . . . . . . . . . 95

4.3.1 Tree biometric characteristics . . . . . . . . . 95

4.3.2 sap flow patterns for Q.i. and Q.p. . . . . . . . 96

4.3.3 Temporal extrapolation of sap flow measurements . 98

4.3.4 Accuracy of the tree classification . . . . . . . . 100

4.3.5 Scalars, scaling-up parameters and BUFs . . . . . . 101

4.3.6 Up-scaled transpiration and spatial patterns . . . . . 103

4.3.7 Comparison of remote-sensing and stand scale scaling up . . . . . . . . . . . . . . 107

4.3.8 Uncertainty analysis of remote-sensing-based scaling up . . . . . . . . . . . . . . . 108

4.4 Conclusions . . . . . . . . . . . . . . . . . . 110

5 Sourcing dry-season tree transpiration 113

5.1 Introduction . . . . . . . . . . . . . . . . . . 114

5.2 Materials and methods . . . . . . . . . . . . . 117

5.2.1 Sourcing of tree transpiration by tracing naturally

abundant isotopes . . . . . . . . . . . 117

5.2.1.1 Sample collection and isotopic content determination . . . . . . . . . . 117

5.2.1.2 Mixing models to calculate proportional contributions . . . . . . . . . . 120

5.2.2 Whole-tree water transport model . . . . . . . . 122

5.2.2.1 Water flow model assumptions . . . . . . . 125

5.2.2.2 Roots-to-canopy water transport . . . . . . 125

5.2.2.3 Stem diameter variation submodel (SDVS) . 125

5.2.2.4 Model implementation . . . . . . . . . . 127

5.2.2.5 Initial conditions and parameter optimisation 127

5.3 Results and discussion . . . . . . . . . . . . 128 
5.3.1 Naturally abundant isotopes in the soil-plant compartments . . . . . . . . . . . . . . . 128

5.3 .2 Mixing-models results . . . . . . . . . . . . . . . . 131

5.3 .3 Sourcing hourly variability . . . . . . . . . . . 136

5.3.4 Explaining water contribution patterns with a tree water flow model . . . . . . . . . . . . . . 137 5.3.4.1 Model calibration and validation for daily sap flow . . . . . . . . . . . . 137

5.3.4.2 Modelling water contribution from the unsaturated and saturated zones of the soil . 139

5.3.4.3 Flow from storage and water potential simulations . . . . . . . . . . . 142

5.4 Conclusions . . . . . . . . . . . . . . . . . . . . . . . 143

5.5 Appendix . . . . . . . . . . . . . . . . . 145

5.5.1 Roots-to-canopy water flow equations . . . . . . . 145

5.5.2 Basic equations for the stem diameter variation submodel (SDVS) . . . . . . . . . . . . . . 152

5.5.3 $\delta^{2} H$ measurements in soil, sap and groundwater . . 155

6 Scaling up water-source contributions to tree-transpiration 157

6.1 Introduction . . . . . . . . . . . . . . . . . 158

6.2 Materials and methods . . . . . . . . . . . . . . . 160

6.2.1 Calculating $T_{g}$ and $T_{u}$ at tree level $\ldots \ldots \ldots 160$

6.2.2 Scaling up $T_{g}$ and $T_{u}$ at the stand and catchment level162

6.3 Results and discussion . . . . . . . . . . . . . . 163

6.3.1 Spatio-temporal patterns of $T_{\text {sta-g }}$ and $T_{\text {sta-u }}$ partitions163

6.3.2 Species-related spatial patterns of $T_{\text {sta-g }}$ and $T_{\text {sta-u }} \quad .165$

6.4 Conclusions . . . . . . . . . . . . . . . . 167

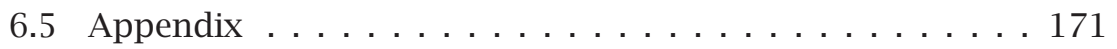

7 Summary 173

7.1 Research setup . . . . . . . . . . . . . . . . . . 174

7.2 Methodological milestones . . . . . . . . . . . 175

7.3 Ecohydrological milestones . . . . . . . . . . 176

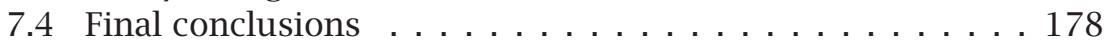

8 Samenvatting 179

8.1 Samenvatting . . . . . . . . . . . . . . . . . 180

$\begin{array}{ll}\text { Bibliography } & 183\end{array}$ 



\section{List of Figures}

1.1 Sunset over the "Dehesa" trees (open oak woodland) in the Sardón study area at the end of summer 2010. . . . . . . . . 1

2.1 Gravimetric water flow tests monitored by infrared imagery.

This Chapter is based on: Novel, cyclic heat dissipation method for the correction of natural temperature gradients in sap flow measurements. Part 2. Laboratory validation. Reyes-Acosta, J.L., M.W. Vandegehuchte, K. Steppe and M.W. Lubczynski. (2012). Published in Tree Physiology. . . . . . . . . . . . . 17

2.2 Diagram of the Mariotte water flow verification system (from Steppe et al. (2010)) used in all experimental set-ups . . . . . 22

2.3 Infrared images monitoring the ATGs produced by the ATG enforcement system on the stem segments during the experiments: (a) shows the stem segments under Mode I and (b) under Mode II. The TDP reference needle is $\approx 10 \mathrm{~cm}$ below the tubing and the heating probe another $10 \mathrm{~cm}$ below. The TDP sensors were intentionally left out of the images (they are in the backside of the stem), to avoid interferences with the observation of the temperature gradients. . . . . . . . . . . 24

2.4 Thermal dissipation probe alternated-power cycle (bold line) with its corresponding extrapolated ad-infinitum transients ON/OFF (dotted lines). Zoomed areas emphasize the position of the variables needed for Eqs. (2.3- (2.7) in the NTG-bias correction. . . . . . . . . . . . . . . . . . . 25

2.5 Test 1: monitoring the ATG with un-powered TDP signal at zero flow. AT, air temperature; $\mathrm{CW}$, circulating water temperature. . . . . . . . . . . . . . . . . 27

2.6 Test 2: impact of ATG upon the Standard-TDP measurements for two stem segments with different sap flux densities (Segment 1 with $J_{p} \approx 17 \mathrm{~cm}^{3} \mathrm{~cm}^{-2}$ hour $^{-1}$ and Segment 2 with $J_{p} \approx 2 \mathrm{~cm}^{3} \mathrm{~cm}^{-2}$ hour-1): (a) raw TDP measurements of $\Delta T$ for both stem segments; (b) comparison of Standard-TDP $J_{p}$ vs. gravimetric control (Grav-Flux). AT, air temperature; CW, circulating water temperature. . . . . . . . . . . 28 
2.7 Test 3: variability of measured raw $\Delta T$ (above) signals and $J_{p}$ (below) for: (i) Standard-TDP (constantly power TDP measurements); (ii) CHD-Flux (ATG corrected), (iii) TTD 2002 (ATG corrected), (iv) TTD 2010 (ATG corrected); vs. (v) Grav-Flux (gravimetric measurements of $J_{p}$ ) as reference. Test 3 was executed under different, gravimetrically enforced sap flux densities: (a) Segment 1 with $\approx 11 \mathrm{~cm}^{3} \mathrm{~cm}^{-2}$ hour ${ }^{-1}$ and (b) Segment 2 with $J_{p} \approx 1 \mathrm{~cm}^{3} \mathrm{~cm}^{-2}$ hour $^{-1}$. The ATG produced during both ATG enforcement modes is plotted as well (Extr.OFF). AT, air temperature; $\mathrm{CW}$, circulating water temperature.

2.8 Boxplot for the corrections obtained during Test 3 vs. the gravimetric reference (Grav-Flux) and uncorrected data (StandardTDP), for (a) Segment 1 and (b) Segment 2. . . . . . . . . . 37

2.9 Accumulated water estimates, calculated for Standard-TDP (constant power) and CHD, TTD-2002 and TTD-2010 for both segments (upper figures $=$ Segment 1 and bottom figures $=$ Segment 2) in ATG Mode I and II, vs. gravimetric reference measurements (Grav-Flux). AT $=$ air temperature and $\mathrm{CW}=$ circulating water temperature. . . . . . . . . . . . 38

2.10 Relative percentage error calculated from gravimetric measurements versus ATG (above) and relative percentage error/residuals in time (below) influencing $J_{p}$ from CHD-Flux, in Segment 1 (a) and Segment 2 (b). The error obtained from the StandardTDP is displayed as an example of a biased signal to highlight the performance of the $\mathrm{CHD}$. $\mathrm{AT}=$ air temperature and $\mathrm{CW}=$ circulating water temperature. . . . . . . . . . . . .

3.1 Sap flow measurements in a Quercus ilex tree during early dryseason (June) at the Sardón catchment in Salamanca, Spain.

This Chapter is based on: Optimization of dry-season sap flow measurements in an oak semi-arid open woodland in Spain. (2014). Published in Ecohydrology. . . . . . . . . . . . 45

3.2 DBH sampling in the Sardón catchment: (a) frequency histogram for DBH size categories; and (b) accumulated frequency curve for DBH size categories obtained from the biometric survey for Q.i. (top) and Q.p. (bottom). . . . . . . . . . 50

3.3 Randomly selected transients (bold lines), modelled (extrapolated dashed lines) to thermal equilibrium in: (a) Q.i. and (b) Q.p. Zoomed areas emphasize the last measured $\Delta T$ at 7.5 min and the extrapolated to thermal equilibrium $\Delta T \ldots \ldots 52$

3.4 Daily variations of NTG acquired by unpowered TDP at different azimuths and different tree heights in Q.i. (a) Northeasterly pattern; (b) South-westerly pattern and (c) BottomUpper patterns at the Northern azimuth. The time axis follows GMT +1 Spanish summer time for all figures. . . . . . . . . 59 
3.5 Daily variations of NTG acquired by unpowered TDP at different azimuths and different tree heights in Q.p. (a) Northeasterly pattern; (b) South-westerly pattern and (c) BottomUpper patterns at the Northern side. The time axis follows GMT+1 Spanish summer time for all figures. . . . . . . . . 60

3.6 $J_{p}$ root mean squared errors (RMSE) between: (1) non-corrected data $\left(\mathrm{TDP}_{s t d}\right)$ and $H F D_{2 \mathrm{~cm}}$; (2) $\mathrm{TDP}_{N T G-\text { free }}$ and $H F D_{2 \mathrm{~cm}}$ (step 1) (3) $\mathrm{TDP}_{\Delta T \text { max -corr }}$ and $H F D_{2 c m}$ (step 2); (4) $\mathrm{TDP}_{\text {final }}$ and $\mathrm{HFD}_{\text {ref }}$ (step 3) for: (a) Q.i. and (b) Q.p. Each bar shows the RMSE for a single tree measured during 2010; the darker gradient of the bars represents smaller DBH and lighter larger DBH. . . . . . . . . . . . . . . . . . . . . 62

3.7 Step-wise optimisation of TDP $J_{p}$ measurements using HFD as reference, for 10 selected $Q . i$. trees $(\mathrm{a}-\mathrm{j})$ : $\mathrm{TDP}_{\text {std }}$ - uncorrected measurement (dotted red line); $\mathrm{TDP}_{N T G-\text { free }^{-} \text {NTG-corrected }}$ $J_{p}$ (dashed violet line); $\mathrm{TDP}_{\Delta T \text { Tax-corr }}$-adjusted for night flow (dashed dark-yellow line); $\mathrm{TDP}_{\text {final }^{-}}$corrected for azimuthal and radial variability $J_{p}$ (light-green line); $H F D_{2 c m}$ - reference for the $2 \mathrm{~cm}$ long TDP sensors (dashed dark-green line); and $\mathrm{HFD}_{r e f^{-}}$(bold blue line) radial-azimuthal $J_{p}$ reference (the time axis follows GMT+1 Spanish summer time). . . . . . . . . . . 63

3.8 Step-wise optimisation of TDP $J_{p}$ measurements using HFD as reference, for 10 selected Q.p. trees $(\mathrm{a}-\mathrm{j})$ : $\mathrm{TDP}_{\text {std }}$ - uncorrected measurement (dotted red line); $\mathrm{TDP}_{N T G-\text { free }^{-} \text {NTG-corrected }}$ $J_{p}$ (dashed violet line); $\mathrm{TDP}_{\Delta T \text { Tmax-corr }}$-adjusted for night flow (dashed dark-yellow line); $\mathrm{TDP}_{\text {final }^{-}}$corrected for azimuthal and radial variability $J_{p}$ (light-green line); $H F D_{2 \mathrm{~cm}}$ - reference for the $2 \mathrm{~cm}$ long TDP sensors (dashed dark-green line); and $\mathrm{HFD}_{r e f}$ - (bold blue line) radial-azimuthal $J_{p}$ reference (the time axis follows GMT +1 Spanish summer time). . . . . . . . . 71

3.9 Average $J_{p-i}$ radial variability of (a) 10 Q.i. trees and (b) 10 Q.p. trees of different DBH sizes measured at the Northern azimuth with the HFD sensor (the time axis follows GMT+1 Spanish summer time). . . . . . . . . . . . . . . . . 72

3.10 Dry season, monthly variability of HFD $J_{p-i}$ measurements (average of 8 measured depths) in: (a) Q.i. and (b) Q.p. for the most representative $\mathrm{DBH}$ size categories, i.e. $\mathrm{DBH}=20-50 \mathrm{~cm}$ (the time axis follows GMT+1 Spanish summer time). . . . . . 72

3.11 HFD azimuthal variability (Northern, Southern, Western and Eastern azimuths) of sap flux density $\left(J_{p-a z}\right)$ as average of 8 measured depths in: (a) 2 Q.i. trees and (b) 2 Q.p. trees, calculated by averaging the azimuthal measurements of four trees per species for the most representative DBH categories ranging from 20 to $50 \mathrm{~cm}$ (the time axis follows GMT+1 Spanish summer). . . . . . . . . . . . . . . . . . 73 
3.12 Optimised $J_{p-i}$ radial variability $\left(\mathrm{TDP}_{\text {final }}\right)$ of 10 Q.i. trees of different DBH sizes measured at the Northern azimuth (a-j). The Figure k shows daily $J_{p-i}$ averages over the 10 trees and the Figure 1 radial depth-wise profiles at 4 different instants (the time axis follows GMT+1 Spanish summer time). . . . . . 74

3.13 Optimised $J_{p-i}$ radial variability ( $\mathrm{TDP}_{\text {final }}$ ) of 10 Q.p. trees of different DBH sizes measured at the Northern azimuth (a-j). The Figure k shows daily $J_{p-i}$ averages over the 10 trees and the Figure 1 radial depth-wise profiles at 4 different instants (the time axis follows GMT+1 Spanish summer time). . . . . . 75

4.1 QuickBird multispectral image highlighting in red the tree canopies of the open-woodland (false-colour near-infrared composite band combination: 4-2-1) at the Sardón catchment in Salamanca, Spain. The location of the Eddy-covariance tower is highlighted in green and the blue lines represent the location of the main water stream.

This Chapter is based on: Mapping dry-season transpiration of an oak wood-land in Salamanca, Spain. Reyes-Acosta, J.L. and M.W. Lubczynski. (2012). Published in Agricultural and Forest Meteorology. . . . . . . . . . . . . . . 77

4.2 "QuickBird" false colour image (Near-infrared composite band combination: 4-2-1) from the Sardón catchment highlighting the infrared portion of the vegetation (vegetation displayed in red) in August $9^{\text {th }}$ of 2009 (The red semi-circles are grasses cultivated for pasture). . . . . . . . . . . . . . . 82

4.3 Variability of daily sap-flux density averaged across xylem depths $\left(J_{p}\right)$ and for $2 \mathrm{~cm}$ depth $\left(J_{p} 2 \mathrm{~cm}\right)$ in function of the the xylem area $\left(A_{x}\right)$ in a.) Q.i. and b.) Q.p. . . . . . . . . . 8

4.4 Summary of the main steps of tree transpiration up-scaling from remotely sensed imagery and GIS techniques. . . . . . . 92

4.5 Cross-correlation matrix of the biometric parameters measured in the survey for (a) Q.i. and (b) Q.p. trees. Diameter at breast height $(D B H)$ is in $\mathrm{m}$, area of the stem $\left(A_{s}\right)$ is in $\mathrm{m}^{2}$, ground-projected canopy area $\left(A_{c}\right)$ is in $\mathrm{m}^{2}$, and xylem area

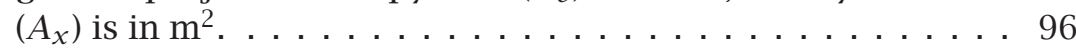

4.6 FITEVAL assessment of the sap-flow simulation results for (a) Cienciala model, Q.i.; (b) Cienciala model, Q.p.; (c) ANN model, Q.i.; (d) ANN model, Q.p. . . . . . . . . . . . . . . . 999

4.7 Dry season temporal sap flow versus climatic temporal variability: (a) daily ANN sap-flow simulations during the dry season of 2009 (15 June - 15 September) for Q.i. and Q.p. trees; (b) incoming solar radiation $(R s)$ and rainfall. . . . . . . 100 
4.8 Biometric-upscaling functions (BUFs) for Q.i. and Q.p., derived from the biometric parameters collected in the tree survey. The BUFs a) and c) follow a $2^{\text {nd }}$ polynomial fitting model for both species respectively, and are useful for ground measurements upscaling. Whereas BUFs b) and d) follow a linear fitting model and are useful for RS upscaling. The respective coefficient of determination $\left(R^{2}\right)$ for each model is provided. . . . . . . . . 104

4.9 Dry-season stand transpiration at 1 ha resolutions ( $\left.T_{\text {sta }}\right)$ upscaled from RS images in the Sardón catchment, compared to the tree density per species in 1 ha per species. . . . . . . . . . . . . . . . . . . . . . . . 105

4.10 Relative frequency distribution of the transpiration at the catchment level $\left(T_{\mathcal{C}}\right)$ obtained from Monte-carlo simulations (1000 iterations) of the RUS technique, for (a) Q.i., (b) Q.p. and (c) for both species (all trees in the catchment). . . . . . . . 109

5.1 Deuterium tracing experiments designed to distinguish water sources of transpiration in a Quercus ilex tree at the end of the dry season 2009 (August).

5.2 Location of isotopic sampling for deuterium measurements. A "QuickBird" false colour image (near-infrared composite band combination: 4-2-1) of the Sardón catchment is used to highlight the infrared portion of the vegetation (trees and grasses displayed in red) on $9^{\text {th }}$ of August 2009 (The red semi-circles are grasses cultivated for pasture) . . . . . . . . 119

5.3 Illustration of the whole-tree water transport model including the unsaturated and saturated zones. This model consists of four compartments, interconnected by three flow paths (i.e. rates of water movement) and controlled by eight inner water potentials. The four compartments were defined as $W_{\text {crown }}$ = crown, $W_{\text {stem }}=$ stem, $W_{\text {root unsat }}=$ roots within unsaturated zone and $W_{\text {root sat }}=$ roots within saturated zone. The four flow rates were defined as $F_{\text {crown }}$ (between $W_{\text {crown }}$ and $W_{\text {stem }}$ ), $F_{\text {stem }}$ (between $W_{\text {stem }}$ and $\left.W_{\text {root unsat }}\right), F_{\text {root unsat }}$ (between $W_{\text {stem }}$ and $W_{\text {root unsat }}$ ) and $F_{\text {root sat }}$ (between $W_{\text {stem }}$ and $W_{\text {root sat }}$ ). The water potentials in the xylem flow compartments were defined as $\Psi_{\text {crown }}^{\mathrm{x}}, \Psi_{\text {stem }}^{\mathrm{x}}, \Psi_{\text {root unsat }}^{\mathrm{x}}$ and $\Psi_{\text {root sat }}^{\mathrm{x}}$. The water potentials in the storage compartments were defined as $\Psi_{\text {crown }}^{\mathrm{s}}, \Psi_{\text {stem }}^{\mathrm{s}}$, $\Psi_{\text {root unsat }}^{\mathrm{s}}$ and $\Psi_{\text {root sat }}^{\mathrm{s}}$. Inner flows into and out of the storage compartment were defined as $f_{\text {crown }}, f_{\text {stem, }} f_{\text {root unsat }}$ and

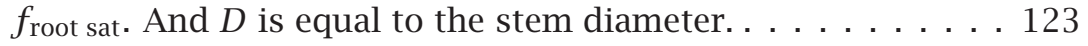


5.4 Electrical representation of the water transport model. Both the xylem and storage main compartments are grouped by dashed-line boxes. The pools are defined as crown, stem, roots in the unsaturated zone (root unsat), and roots in the saturated zone (root sat). The driving variables of the model are as follows: water content in a storage compartment ( $\left.W_{\text {pool }}\right)$; water potential in a storage compartment $\left(\Psi_{\text {pool }}^{\mathrm{s}}\right)$; water potential in a xylem compartment ( $\Psi_{\text {pool }}^{\mathrm{x}}$ ); water flow exchange between the xylem and the storage compartment $\left(f_{\text {pool }}\right)$; water flow through a xylem compartment $\left(F_{\text {pool }}\right)$; flow resistance in between a xylem and a storage compartment $\left(R_{\text {pool }}^{\mathrm{s}}\right)$; flow resistance between xylem compartments $\left(R_{\text {pool-pool }}^{\mathrm{x}}\right)$; and capacitance of the storage compartment $\left(C_{\text {pool }}\right) . \ldots \ldots \ldots \ldots$

5.5 Average isotopic readings from two subsurface sources (soil layers and groundwater) and from the xylem of tree compartments representing an isotopic mixture, measured in the early period of the dry-season referred to as WET (May-June 2010) and in the late period of the dry-season referred to as DRY (August-September 2009) on a Q.i. tree (a. and c.) and on a Q.p. tree (b. and d.). The error bars show the standard deviation. 0 cm in the y axis refers to the ground level. . . . . . . . . 129

5.6 Boxplot of the contribution fraction for a.) Q.i. and b.) Q.p. in the WET period (June 2010); and c.) Q.i., and d.) Q.p. in the DRY period (August-September 2009). . . . . . . . . . . . . 133

5.7 Soil moisture profile at four depths for a Q.p. tree monitored during the fieldwork campaign of 2009. Y axis represents \% of water content in the unsaturated layers. This tree was located further from the river and thus its profile differs slightly from that of the Q.p. tree in which the isotope tracing was made. However, it does show how deeper soil layers (75 to $90 \mathrm{~cm}$ deep) are kept with relatively high moist throughout the dry season, and how only at the very end (of the DRY period) the effect of the shallow groundwater diminishes and drier conditions prevail. . . . . . . . . . . . . . . . 135

5.8 Time series of feasible contributions from each source to the sap water extracted from the tree every three hours (three samples per hour), in (a) Q.i. and (b) Q.p.. The time follows GMT+1 Spanish summer time, which is $2.5 \mathrm{~h}$ ahead as compared with geographical (solar) time. . . . . . . . . . 136

5.9 Figure (a) illustrates the field sap flow data (MeasSap) and the simulation after convergence (SimSap). Figure (b) shows the climatic drivers used for simulation, shortwave incoming radiation (green line), air temperature (red line), vapour pressure deficit (VPD) (cyan line) and soil water potential at $50 \mathrm{~cm}$ (dark blue line). . . . . . . . . . . . . . . . . . 138 
5.10 Saturated zone $\left(T_{g}\right)$ and unsaturated zone $\left(T_{u}\right)$ contribution simulations for a typical Q.i. tree with a $23 \mathrm{~cm}$ diameter at breast height under soil moisture conditions of the (a) DRY and (b) WET periods. . . . . . . . . . . . . . . . 140

5.11 This figure shows the simulations run for Q.i. in the DRY (left) and WET (right) period. a) and b) show the stem flow simulations. c) and d) show three-day zoomed-in view of the stem flow (coloured lines) and VPD (black line). e) and f) show the storage water flows in (positive) and out (negative) of the storage compartments. g) and h) show the diameter variation of the trunk. i) and j) show the hourly variation of the water potential $(\Psi)$ defining the forces driving the water flow and diameter variation dynamics in the considered compartments. 144

6.1 Patches of Quercus ilex and Quercus pyrenaica trees in the Sardón catchment during the dry season in September 2009.

As observed, grasses and herbaceous plants have all wilted away, indicating water deficit in the soil, but the foliage of oak trees displays a lush green colour. . . . . . . . . . . . . . 157

$6.2 T_{\text {sta-g }}$ and $T_{\text {sta-u }}$ distribution in the Sardón catchment during the early (WET) and late (DRY) dry season periods at 1 ha grid resolution upscaled from using RS techniques on highresolution images and naturally-abundant isotopes tracing. . . 169

6.3 Species dependant $T_{\text {sta-g }}$ and $T_{\text {sta-u }}$ distribution in the Sardón catchment during the early (WET) and late (DRY) dry season periods at 1 ha grid resolutions. Due to space constraints the map legends have not been included, but grid colours follow the same values and gradients as maps in Figure 6.2. These maps were calculated by separating Q.i. and Q.p. sap flow measurements and the applying Eq. $4.8-4.10$ as described in Chapter $4 \ldots \ldots \ldots \ldots$. . . . . . . . . . . 170

6.4 Deuterium and sap flow measurements during WET (a. Q.i. and b. Q.p.) and DRY (c. Q.i. and d. Q.p.) periods in trees of the most typical size in Sardón. The reduction in the isotopic concentration of the soil from WET (higher humidity and higher water table) to DRY (lower humidity and lower water table) indicates a shift in soil moisture content and thus of water consumption. Coloured lines represent the different water sources sampled (soil depths, groundwater and sap water), and the black line is sap flow. Time axes follow Spanish summer time, GMT +1 , which is approximately 2.5 hours ahead of the geographical ("solar") time. . . . . . . . . . . . . . . 171 



\section{List of Tables}

2.1 Biometric measurements of the stem segments of European beech (F. sylvatica) used in the tests . . . . . . . . . . . . . 21

2.2 Basic statistics of sap flux density measurements and estimates for Test 2 excluding stabilization period. . . . . . . . 35

2.3 Basic statistics of the sap flux density measurements and estimates for Test 3, using different correction methods: CHDFlux $7.5 \mathrm{~min}$; TTD $7.5 \mathrm{~min}$; and TTD 10-20 min. Segment $1=$ $11 \mathrm{~cm}^{3} \mathrm{~cm}^{-2}$ hour ${ }^{-1}$ and Segment $2=1 \mathrm{~cm}^{3} \mathrm{~cm}^{-2}$ hour $^{-1}$. . 37

3.1 Stratified sampling scheme applied for the dry-season sap flow campaigns of 2009 and $2010 \ldots \ldots \ldots$. . . . . . . . 51

3.2 Biometric characteristics of the 10 sampled trees of Q.i., and 10 sampled trees of Q.p. in the Sardón catchment 2009-2010. 58

3.3 Min-max range of the CHD fitting parameters for Q.i. and Q.p. 61

4.1 Average $J_{p i}$ profile for the first two centimetres $(0.5$ to $1.5 \mathrm{~cm})$ in the tree-size categories for Q.i. and Q.p. . . . . . . . 85

4.2 Main statistics of the biometric parameters obtained in the survey for Q.i. $(n=90)$ and Q.p. $(n=84) \ldots \ldots \ldots \ldots$

4.3 Error matrix of 800 randomly selected ground-control points (canopies) used for the calculation of the error indexes of the tree-species classification map. . . . . . . . . . . . . . . . 101

4.4 Pearson product-moment correlation coefficient $(r)$ matrix for the surveyed biometric parameters in Q.i. and Q.p. . . . . . . 102

4.5 Comparison between upscaling techniques for $T_{\text {sta }}$ at 1 ha selected from the biometric ground measurements. . . . . . . 108

5.1 Average meteorological conditions during isotopic sampling, from an automatic data acquisition station in the proximity of the sampled trees. $\mathrm{RH}=$ relative humidity, $\mathrm{VPD}=$ vapour pressure deficit, iSR $=$ incoming solar radiation and Ws $=$ wind speed. . . . . . . . . . . . . . . . . . . . . 117

5.2 Initial guess for parameter optimisation of resistances and capacitances, together with optimised values for DRY and WET. Capacitances $\left(C_{\text {pool }}\right)$ units MPa g ${ }^{-1}$ and Resistance $\left(R_{\text {pool-pool }}^{X}\right)$ units MPa $\mathrm{s} \mathrm{g}^{-1} \ldots \ldots \ldots \ldots \ldots \ldots \ldots \ldots \ldots \ldots \ldots$ 
5.3 Average source contributions to sap-water calculated from Eq. 5.4, for the 24 hours sampling at 4 soil-water layers and groundwater derived from $\delta^{2} H$ mixing models analysed with Iso-source, in WET period of dry season 2010 (Mixir) and DRY period of dry season (IsoSource) 2009. . . . . . . . . . . . 132

5.4 Symbol, unit and description of the model variables. Pools (pool) are defined as crown, stem, roots in the unsaturated zone and roots in the saturated zone. . . . . . . . . . . . . . 146

5.5 Symbol, unit and description of the model parameters. Pools are defined as crown, stem, roots in the unsaturated zone and roots in the saturated zone. . . . . . . . . . . . . . . . 147

5.6 Deuterium concentrations in the sampled compartments in 2009 and 2010 for Q.i..The results of the sampling are expressed as the hydrogen isotope ratio $\left(\delta^{2} H\right)$ in parts per thousand (\%) relative to the accepted standard (V-SMOW). GW = Groundwater sample and SAP $=$ sapwater sample $\ldots . . . .155$

5.7 Deuterium concentrations in the sampled compartments in 2009 and 2010 for Q.p..The results of the sampling are expressed as the hydrogen isotope ratio $\left(\delta^{2} H\right)$ in parts per thousand (\%) relative to the accepted standard (V-SMOW). GW = Groundwater sample and SAP $=$ sapwater sample $\ldots \ldots \ldots 156$

6.1 Water source contributions used to account for temporal variability in the estimations of $T_{\text {sta }}$ and $T_{c} \ldots \ldots \ldots \ldots 162$

6.2 Temporal variation of $T_{\text {sta-g }}, T_{\text {sta-u }}$, and $T_{\mathcal{C}}$ in the Sardón catchment . . . . . . . . . . . . . . . . . . . 164

6.3 Total volume of water (litres day ${ }^{-1}$ ) uptaken from the groundwater $(g)$ and unsaturated zone $(u)$ of al trees per species for the whole Sardón catchment $(\approx 7,546$ ha), during the WET and DRY periods in the dry-season. Min. is always zero for all cases. Max. is the maximum value recorded in a grid, Sum. is the aggregation of all cells in the grid, Mean is the average value for the grid, and SD. is the standard deviation. . . . . . 166 


\section{Tree-water interactions in water limited environments}

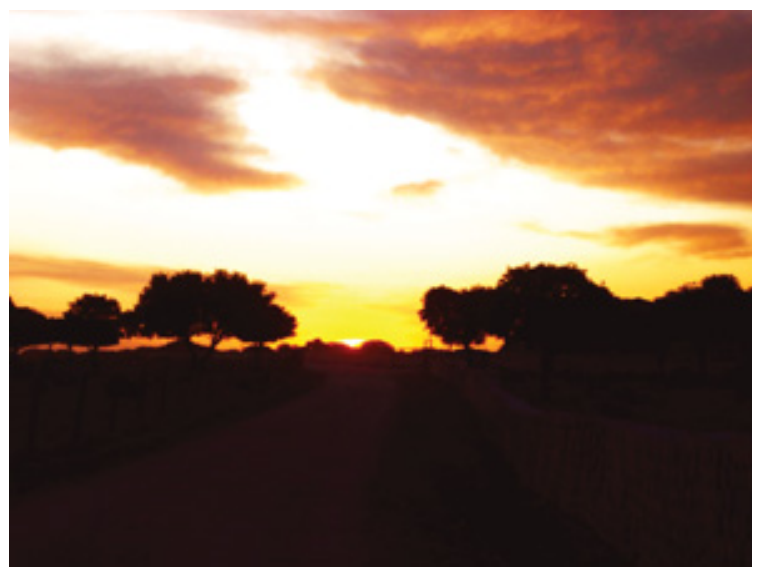

Figure 1.1: Sunset over the "Dehesa" trees (open oak woodland) in the Sardón study area at the end of summer 2010. 


\subsection{Introduction}

\subsubsection{The role of transpiration in ecohydrological systems}

Plant transpiration is a biological process of water vapour loss that is part of the water cycle. During transpiration water is transported through plants from the roots to the stomata on the underside of leaves, where it is converted into vapour and released into the atmosphere (Cummins, 2007; USGS, 2012; Martin et al., 1976; Allen et al., 1998).

This process is possible thanks to the opening of the stomata that are linked to the photosynthetic activity requiring $\mathrm{CO}_{2}$ gas to enter the pores in the leaves (Sadava et al., 2009). During the opening of the stomata moisture is lost from the tissues in the stomata and as a by-product of the photosynthesis itself (Sadava et al., 2009). Transpiration occurs in other parts of the plants as well (e.g. roots and flowers) but it is negligible compared with the water loss from stomata (Cummins, 2007; USGS, 2012).

The loss of mass of water, driven by the diffusion of water from the stomata into the atmosphere, generates a water flow caused by a decrease in the water pressure (water potential) in the upper parts of the plant (Martin et al., 1976; Tyree and Zimmerman, 2002). The decrease in the water pressure creates a water potential gradient through the conductive tissues in the plant (xylem or sapwood), providing the hydrostatic energy to absorb water from the soil through the roots (Tyree and Zimmerman, 2002). This movement of water has been defined as sap flow.

Approximately $90 \%$ of the water absorbed by plants is used in transpiration (USGS, 2012; Cummins, 2007). Furthermore, up to 10\% of the water in the atmosphere comes from plant transpiration (USGS, 2012), thus indicating that this is the world's most important biological water control mechanism.

Vegetated ecosystems are intimately regulated by the plant-water-soil interactions. The vegetation controls the amounts of water that has infiltrated to the deep layers of unsaturated and saturated zones of the subsurface (Brown et al., 2005; Ghimire et al., 2012), the circulation of nutrients in the soil (Allen and Chapman, 2001), the soil moisture (Hernández-Santana et al., 2008a), and even the groundwater dynamics (Lubczynski, 2009, 2000). All these mechanisms are bound to hydrological dynamics but transpiration is the main mechanism of water use by vegetation. The mechanisms of water-use regulation are especially striking in environments where water is the limiting factor, and the vegetation has evolved to adapt to this limitation (Rodriguez-Iturbe et al., 2001). Such environments are called water limited environments (WLEs) (Abrahams and Parsons, 1994; Guswa et al., 2004) because the potential evapotranspiration highly surpasses the rainfall rate. In such environments water availability shapes not only the distribution of the vegetation but also the ecosystem itself.

The vegetation in such areas has developed different adaptations and strategies concerning water use, because water deficit is often the 
most important stress factor for vegetation (Porporato et al., 2001). Such strategies include water redistribution (Brooks et al., 2002), storing water and nutrients in tissues, specialised photosynthetic pathways, "behavioural" stomatal control adaptations, and diverse rooting patterns (Noy-Meir, 1973). These strategies guarantee a quasi-continuous water supply throughout the whole year, and are evident in the year-round "green-state" of certain plants, even in drought periods (Noy-Meir, 1973).

The mentioned adaptations and transpiration strategies importantly influence the hydrological dynamics of WLEs, as they determine the regulation of an important amount of water in the hydrological system (recharge-discharge dynamics), especially for aquifers (i.e. groundwater reservoirs) (Rodriguez-Iturbe et al., 2001). Moreover, as recharge is very low in the majority of WLEs, even small rates of water loss due to groundwater transpiration (Lubczynski, 2009) can be significant.

As water resources are becoming scarcer around the world (Hanasaki et al., 2013), a proper quantification of transpiration is critical to understanding the movement of water in the environment, especially in the WLEs. Moreover, the evidence of accelerated global warming highlights the need for a deep understanding of the WLEs, because an important proportion of the world population lives in such areas, and these areas are also tending to expand quicker than others (Hanasaki et al., 2013; Schewe et al., 2014).

These pressing concerns have triggered an increasing scientific interest in ecohydrological functioning of WLE's and water resource management over the last decades (Newman et al., 2006). Furthermore, several computational tools have been exclusively developed to explore and understand different hydrological processes (see Baird and Maddock, 2005; Batelaan and De Smedt, 2007; Batelaan et al., 2003; Oltchev et al., 2002a; Francés et al., 2011).

As more knowledge is produced, new features - too complex to be taken into consideration in the past - are now being studied thanks to the advances in technology and computational capacity (Dale, 2003). As highlighted by Newman et al. (2006) regarding ecohydrological research in WLE's: "the undertaking of eco-hydrological issues proposes a scientific challenge in which hydrology and ecology must be combined to understand the environmental systems in a more integrated or comprehensive way".

One of the most challenging aspects for future ecohydrological research is to understand the role of the vegetation in WLEs. This is because in such environments the overall quantities of water involved in subsurface water circulation are generally low or very low, due to the low precipitation and high evapotranspiration (Noy-Meir, 1973; RodriguezIturbe et al., 2001).

The amounts and sources of water that the vegetation uptakes at different spatiotemporal scales are essential information that are necessary to properly study the role of the vegetation in WLEs. Such information would offer a deeper understanding of the characteristics and connections of different hydrological compartments (e.g. soil, groundwater), at 
different spatial and temporal scales. In order to provide such information for complex vegetation arrangements and to investigate the main ecohydrological connections, processes, inputs, outputs and properties of WLEs, the application of a series of complementary methodologies with a holistic approach is necessary. In this line of thinking, the first step is to quantify transpiration fluxes for a single tree. This quantification requires the use of a methodology with sufficient accuracy to provide a realistic estimation of water abstraction at the tree scale. The second step is to properly cover the water abstraction variation within the studied population, as trees might have different characteristics and water consumption rates/requirements.

The third step consists of a procedure in which the obtained measurements can be efficiently extrapolated from the sampled trees to forest stands and even catchments. This requires the quantification of trees at the selected spatial scale and the definition of enough characteristics of each tree to extrapolate the transpiration measurements. The fourth step requires the development and use of a methodology to source or trace the origin of the water in different compartments of individual trees and evaluate their contributions to the total transpiration flow, presenting such information at different spatiotemporal scales. The final step requires an in-depth explanation of the control mechanisms generating the observed and quantified patterns, in a way that the obtained information can be of most use for hydrological models and consequently for water resource management.

The exploration and development of the above-described steps required the selection of a WLE study area where the following characteristics were available: presence of phreatophyte trees, easy access for experimentation and equipment installation, low level of human intervention, relatively non-complex ecosystem (e.g. low plant diversity), and limited water availability.

Therefore, the Sardón catchment $\left(\approx 80 \mathrm{~km}^{2}\right)$ located west of Salamanca, Spain, was selected as an ideal study area because of the type of open woodland systems (i.e. "Dehesas") that were found there. Furthermore, this kind of ecosystem is of high interest to communities and governments preserving and managing Mediterranean areas of Europe (David et al., 2004; Moreno et al., 2005; Smit et al., 2008). Moreover, its climatic conditions during the dry season are comparable to those of many other WLE areas around the world, and thus the knowledge obtained from this research can be transferable to such areas.

This $\mathrm{PhD}$ dissertation provides extensive information and detailed guidelines regarding the above-mentioned steps, as well as a comprehensive explanation of the hydrological dynamics and roles of trees in a WLE. 


\subsection{Scientific considerations}

\subsubsection{Brief overview of sap flow measurements}

Sap flow measurement is an in situ methodology to measure the flowing water in the conductive tissues of plants. It is one of the most widely used methodologies because it enables direct field measurements, simultaneous monitoring of several trees and, if used properly, provides an appropriate quantification of transpiration (Lu et al., 2002).

The majority of sap flow methods use thermal principles to relate heat processes with water flow, either empirically (e.g. Granier's probe (Granier, 1985, 1987) and heat balance (Čermák et al., 1973; Kučera et al., 1977)), semi-empirically (e.g. heat field deformation method (Nadezhdina et al., 2002)), by measuring pulses (e.g. heat pulse method and heat ratio method (Burgess et al., 2001)), and more recently by using physicallybased heat principles (e.g. Sapflow+ (Vandegehuchte and Steppe, 2012)).

The diversity of thermal sap flow methods provides flexibility with respect to costs and applications; however, there is no single method combining high accuracy and efficiency.

For example, the thermal dissipation probe (TDP) of Granier (1985, 1987 ) is the most popular and widely used due to its simplicity and low cost. However, in its original implementation the TDP is prone to natural thermal gradient (NTG) biases, is affected by the radial heterogeneity of sap flow, and cannot measure reverse flow.

In comparison, the heat field deformation (HFD) method provides a higher number of measuring points (eight) up to $7.5 \mathrm{~cm}$ deep, thus offering an augmented and sophisticated perspective on the radial water flow variation within the sapwood tissue. HFD is also sensitive to very low backward/forward flows, which is advantageous for measuring nocturnal flows. However, such sensors are costly and difficult to produce, which limits their applicability when several trees need to be measured simultaneously. Moreover, it has been observed that HFD is also sensitive to NTGs (Reyes-Acosta and Lubczynski, 2014), but the biasing effect of this sensitivity has not yet been analysed.

Other methodologies that make use of thermal principles to define sap flow velocities and/or flows have limitations or biasing effects comparable to those of the TDP and HFD. Among the most well-known methodologies are the heat balance method (HBM) (Grime and Sinclair, 1999), the compensation heat pulse method (CHPM) (Edwards et al., 1997), the heat ratio method (HRM) (Burgess et al., 2001), and the tissue heat balance method (THB) or Čermák-type sensors (Köstner et al., 1998).

Other approaches indirectly estimate $\mathrm{T}$ as a difference from actual ET measurements, typically obtained from scintillometry methods. Such ET measurements are acquired using eddy-covariance (Yaseef et al., 2010) or Bowen- ratio (Brunel et al., 1997) methodologies, amongst others. Such approach, is mostly suitable for homogeneous forest stands or larger spatial scales. Thus scintillometry applications on patchy landscapes with large surface-roughness variation like in Sardón, are 
more susceptible to interpretation errors (Finnigan, 2004; Göckede et al., 2004). Therefore the flexibility of tree-scale sap flow measurements is more adequate to determine $\mathrm{T}$ in heterogeneous landscapes.

Thus, together with the mentioned technical limitations, the underlying challenges when using sap flow methodologies to measure water use in trees are to: (1) ensure a high level of accuracy free from biases, (2) account for radial variability of the flow in the xylem, (3) account for circular variability, (4) account for reverse flows or continuous nocturnal flows, and (5) account for storage effects if these are large. Therefore if sap flow methodologies need to be used to quantify tree water use in a WLE, all the previously mentioned aspects must be taken into consideration.

\subsubsection{Methods to scale up sap flow measurements to tree transpiration at different spatial scales}

It is possible to calculate the transpiration of single trees if the sap flow methodologies are appropriately used. Such measurements are valuable in understanding the physiological processes driving the water use in trees and their water-use consumption patterns. However, this information is limited to the number of monitored trees. Hence, if the objective of the study requires quantifications of transpiration at scales larger than the tree scale, the transpiration measurements at the tree level must be scaled up.

A set of methodologies have been proposed to extrapolate sap flow data from single trees to higher spatial scales (Hatton and Wu, 1995; Čermák et al., 1998; Čermák and Nadezhdina, 1998; Köstner et al., 1998; Čermák et al., 2004). These methods are generally known as "scaling-up" methodologies (Lubczynski, 2009) and have the following aspects in common: (1) they require the application of an appropriate sampling methodology to account for the tree size variability when measuring the sap flow, (2) they establish a mathematical relation (called a scaling-up function) of a scaled-up parameter (sapwood area or sap flow) with an easily measurable tree characteristic (called a scalar), and finally (3) they measure scalars at spatial scales larger than the scales of data acquisition to be converted to scaled parameter applying a scaling-up function.

The scaling-up techniques have been developed to deal with specific forest conditions. If the forest has a high density of trees and the canopies are overlapping, the selected scalars can be the basal area (Čermák et al., 2004), the leaf area (Hatton and Wu, 1995) or the diameter at breast height (Granier, 1996), to name the most commonly used.

These techniques have been classically used to calculate the water used in forest stands. However, when higher scales (e.g. catchment scale) are involved in the analysis, they are not logistically efficient because of the high number of trees that have to be measured. This is especially critical in open forests and savannahs where trees can be widely spread, thus increasing sampling effort. 
Another technique that has been recently explored for large scales is to derive a direct relationship between evapotranspiration and the reflectance of the vegetation from remotely sensed images of large areas (Boegh et al., 1999; Nagler et al., 2007; Murray et al., 2009; Cristóbal et al., 2011). But this alternative currently has an error of 20 to 30\% and does not differentiate between the evaporation and transpiration of vegetated areas (i.e. non-bare-soil areas). Thus, a direct scaling up of sap flow is still the most accurate option for estimating transpiration at large spatial scales (e.g. forest stands).

An alternative to combining the direct scaling up of sap flow measurements using remote sensing techniques is to use object attributes derived from the remote sensing images. This method was originally derived by Chavarro-Rincón (2009) and is based on mathematically relating the projected canopy areas of single trees with sap flow to calculate the overall transpiration. This is a very efficient and convenient solution for ecosystems with sparse vegetation.

In this study the scaling-up methodology proposed by ChavarroRincón (2009) was modified (instead of sap flow, sapwood area was scaled) to integrate the sap flow azimuthal/radial variability and the sap flow tree size variability. The aim of this integration was to minimise the effect of the sap flow variability on the transpiration estimations. In order to do this, the projected canopy areas were used to estimate the sapwood areas (by establishing an a-priori mathematical relation), and then a depth-wise set of sap flow values were assigned to each tree depending on the stem cross-sectional areas.

Finally, the uncertainty and error propagation of the remote sensing scaling-up procedure was evaluated in order to determine its accuracy, and thus its potential, for future transpiration estimations in other water limited catchments.

\subsubsection{Water balance, transpiration and naturally abundant isotopes for water sourcing}

Since scientists have clearly shown the importance of vegetation in WLEs, the demand for groundwater transpiration quantification has increased. However, groundwater transpiration $\left(T_{g}\right)$ quantification with high temporal resolution is technically difficult (Lubczynski, 2009).

The general water balance consists of five elements defined by the following equation:

$$
\frac{\delta S}{\delta t}=P-E T-Q_{d}-\Delta Q_{g}
$$

where $(\delta S)$ is the change in storage, $P$ is precipitation, ET is evapotranspiration , $Q_{d}$ is direct run-off consisting of surface and subsurface runoff, and $\Delta Q_{g}$ is net groundwater flow (Freeze and Cherry, 1979). These elements are mathematically related in a conservative system (i.e. linear and energy preserving), where change of $\delta S$ in time depends on the balance of the water input $(P)$ and outputs $\left(E T, Q_{d}\right.$ and $\left.\Delta Q_{g}\right)$. 
As illustrated in Eq. 1.2 ET can be further split into two components: surface evapotranspiration $\left(E T_{S}\right)$ and subsurface evapotranspiration $\left(E T_{s s}\right) . E T_{S}$ consists of elements related with surface processes such as interception, direct evaporation from terrain surface, and evaporation from water bodies. $E T_{s s}$ comprises unsaturated zone evapotranspiration $\left(E T_{u}\right)$ and groundwater evapotranspiration $\left(E T_{g}\right)$, as defined in Eq. 1.3. Likewise, $E T_{u}$ consists of transpiration from the unsaturated zone $\left(T_{u}\right)$ by root water uptake, and evaporation from the unsaturated zone $\left(E_{u}\right)$ by upward flux originating from soil moisture in the unsaturated zone, excluding the capillary zone (see Eq. 1.4). $E T_{g}$ consists of transpiration from groundwater $\left(T_{g}\right)$ by deep root system uptake, and evaporation from groundwater $\left(E_{g}\right)$ by upward flux originating in the saturated zone and/or capillary zone (see Eq. 1.4) (Lubczynski and Gurwin, 2005; Lubczynski, 2009).

$$
\begin{aligned}
& E T=E T_{s}+E T_{s s} \\
& E T=E T_{s}+E T_{u}+E T_{g} \\
& E T=E T_{s}+\left(E_{u}+T_{u}\right)+\left(E_{g}+T_{g}\right)
\end{aligned}
$$

From this rationale, it can be inferred that storage in any aquifer is affected by the described processes. Hence, different aquifers will be influenced by various combinations of unsaturated zone and groundwater evapotranspiration regimes. For example, it has been demonstrated that in WLEs, $E T_{g}$ (Eq. 1.3) constitutes one of the main components of the groundwater balance (Lubczynski, 2000; Lubczynski and Gurwin, 2005). During dry seasons $E T_{s} \approx 0$ and $E T_{u}$ quickly declines due to the large vapour pressure deficit. For example, in the case of shallow water table conditions and an unsaturated zone with very low field capacity, the assumption that $E T_{s} \approx 0$ and $E T_{u}$ is low or negligible (due to low field capacity) is well justified, implying that the dry-season ET is mostly driven by $E T_{g}$.

$E T_{g}$ combines the processes of groundwater transpiration $\left(T_{g}\right)$ and groundwater evaporation $\left(E_{g}\right)$ - both very important in groundwater resources evaluation. The focus of this study, however, is dedicated mainly to total transpiration $(T)$ and $T_{g} . T_{g}$ is part of the $T$, as defined by Equation 1.5 .

$$
T=T_{u}+T_{g}
$$

$T$ is well measurable by sap flow $\left(Q_{S}\right)$, if properly sampled, recorded, and constrained to determine the inner sapwood variabilities and the tree size variabilities. However, the technique to define the contributions (i.e. 
sources) of the two components ( $T_{u}$ and $T_{g}$ ) is currently a scientific and technical challenge (Lubczynski and Gurwin, 2005; Lubczynski, 2009), and is one of the main tasks of this study.

Various studies have focused on defining appropriate means for measuring or calculating $T_{g}$ (Baird and Maddock, 2005; Cook and O'Grady, 2006; David et al., 2007, 2004; Lubczynski and Gurwin, 2005; Lubczynski, 2000; Zencich et al., 2002; Obakeng et al., 2007; Scanlon et al., 2002; Thorburn et al., 1993). However, few results have been quantitatively conclusive in sourcing $T$ into $T_{u}$ and $T_{g}$.

Stable isotope methods have recently emerged as one of the more powerful tools for advancing understanding of relationships between plants and their environment. These methods provide insights into the movement of water and nutrients across a range of spatiotemporal scales and ecohydrological systems, facilitating research into highly complex issues (White, 1989; Nadelhoffer and Fry, 1994; Dawson et al., 2002). In this regard, naturally abundant isotopes are used as integrators (evaluation of net outcomes from processes) and tracers (to follow the fates and transformation of a resource) of an ecological process (Dawson et al., 2002). This technique is being widely used in plant resources acquisition, especially as regards three key biological elements: water, nitrogen and carbon (Dawson et al., 2002).

Stable isotope analysis of water sources is mainly applied to define isotopic ratios of $\delta \mathrm{D}$ (Deuterium) and $\delta^{18} \mathrm{O}$ (Oxygen 18) as tracers (Wershaw et al., 1966). Water sources are specific "pools" or "water-containers" available in the natural systems (e.g. soil layers, groundwater, river beds, etc.), where plants abstract water to be used in physiological processes (Dawson et al., 2002). As regards terrestrial plants, if samples of different water sources can be obtained and the plant's xylem sap extracted, it is possible to assess which sources of water are being used and quantify the amount of water abstracted (Brunel et al., 1995; Ehleringer et al., 2000; Thorburn et al., 1993; Turner et al., 2001).

Recently, isotope analyses have been used for defining groundwater use (Cook and O'Grady, 2006; David et al., 2007). Nevertheless, for tracers to be useful there must be no isotopic fractionation, no mixture of water sources during movement to the plant, and the water sources must have significantly different isotopic compositions (Dawson et al., 2002). Fulfilling these requirements can be difficult if the sampling or analytic technique allows evaporation of the liquid samples (i.e. enrichment due to water loss), and if the isotopic compositions of the water sources have been homogenised by any natural process (e.g. water-soil compartments and shallow groundwater can have an identical isotopic composition after strong rain events or if close to riverbanks) (White, 1989; Zencich et al., 2002; Phillips and Gregg, 2003). Even when there is no fractionation, it has been suggested that the mixed-source results might be misleading, mainly because $\delta \mathrm{D}$ and $\delta^{18} \mathrm{O}$ are highly correlated or the sources were isotopically not sufficiently mutually exclusive. For example, Phillips and Gregg (2003) found various possible solutions to source combinations when numerically analysing a one-isotope mixing model applied to water 
sourcing research (Zencich et al., 2002). Phillips and Gregg (2003) and Moore et al. (2008) have both suggested statistical methods to account for the uncertainty of the isotopic mixed-source solutions, thus providing better informed and more accurate results.

\subsubsection{Tree water flow modelling and sourcing of water contributions}

Several studies have attempted to indicate the water contributions from diverse sources to the overall water uptake in plants, especially in drier conditions (Thorburn et al., 1993; Zencich et al., 2002; Phillips and Gregg, 2003; David et al., 2007). However, such studies have not elaborated on the spatiotemporal variability of these contributions from the analysed soil-water compartments (i.e. unsaturated and saturated zones in the soil profile), nor which mechanisms determine them.

The plant water contributions from subsurface compartments and their variability is driven by diverse tree physiological and non-physiological factors. However, in WLEs, where water availability is the main limiting factor, the most likely driving force is water potential (Tyree and Zimmerman, 2002). The water potential defines the increment and decrement of the resistivity in the conductive compartments of the plants, which ultimately determines the water flow.

This mechanism of water control is very intricate and complex, especially when considering fully grown trees with dense canopies and deep root systems. Thus it is extremely challenging (technically and logistically) to simultaneously measure all the resistances and water potentials affecting the water flow in a single tree to obtain a full understanding of the water flow dynamics.

The most convenient alternative to in-situ measurements is to use a modelling technique capable of integrating biological and hydrological processes operating in the roots, the stem and the canopy, but at the same time simple enough to provide a clear overview of the most important factors controlling the water uptake in the saturated and unsaturated zones of the subsurface.

Several modelling efforts have been made since the early 80 s to efficiently and realistically model the water flow dynamics in plants (Edwards et al., 1986; Tyree, 1988; Tyree et al., 1991; Williams et al., 1996, 2001; Tyree and Zimmerman, 2002; Steppe et al., 2006; Steppe and Lemeur, 2007a). Therefore, there is a comprehensive and reliable number of models that can be combined and used to model the water flow dynamics. The remaining challenges to explain the water contributions of the subsurface compartments when using such modelling techniques are to: (1) define the appropriate mechanism to relate the subsurface root compartment system to the tree compartment, (2) determine and calibrate the parameters governing the water dynamics between and within compartments, and (3) provide a realistic approximation of water potentials and flows. 


\subsection{Problem statement and related novelty}

The vegetation in WLEs is important for the regulation of the hydrological processes and these processes require further studying. Therefore, this research focused on defining, understanding and quantifying the soil water and groundwater uptake dynamics of the phreatophytic vegetation of the oak trees Quercus pyrenaica and Quercus ilex in the Sardón catchment in Salamanca Province, Spain. Furthermore, this research aimed to increase understanding of the relationship between tree water uptake and water-use strategies in a spatially explicit context.

In order to meet these aims, it was first necessary to deal with a series of technical and methodological aspects regarding the measurement of water flowing through the sapwood of trees (i.e. sap flow) (Chapters 2 and 3). Second, to understand the spatial implications of the transpiration dynamics, it was necessary to determine an appropriate technique to efficiently and accurately estimate sap flow at large scales (Chapter 4). Finally, by combining the spatially explicit data of large-scale transpiration with the results of naturally abundant-isotope tracing experiments and plant physiology modelling, a comprehensive explanation of the water abstraction dynamics and strategies was proposed (Chapters 5 and 6). The following subsections describe the specific challenges and the related novelty of each stage of this thesis.

\subsubsection{Natural temperature gradient bias on sap flow measurements}

The Granier-type sap flow sensors (TDP) have low production costs and are reliable. However, the main limitation to their use in WLEs is their high sensitivity to natural temperature gradients biases. As the TDPs use a thermal method to empirically relate the water flow magnitude to temperature difference in the conductive tissue of the plants, even a slight temperature change can generate significant overestimations and underestimations. Therefore, natural thermal gradients - thermal processes that affect all surfaces susceptible to temperature change in any environment - are one of the most common biasing factors for thermal methods of sap flow measurement.

In WLEs during the dry season, NTGs are even more apparent because of the highly contrasting night and day temperatures that enforce the formation of the gradients in the tree trunks. Despite the big issues that this represents for sap flow measurements with TDPs, there is little understanding of the process triggered by NTGs affecting TDP sensors. Moreover, proposed technical solutions such as the transient thermal dissipation (TTD) method of Do and Rocheteau (2002a,b) and the cyclic heat dissipation (CHD) method of Chavarro-Rincón (2009) and Lubczynski et al. (2012) unfortunately lacked cross-validation under homogeneous conditions.

Therefore, the efforts at this stage of the doctoral study were geared to providing the first (to the author's knowledge) laboratory-controlled cross-validation of the TTD and CHD methods, together with a thorough 
explanation of the NTG biasing dynamics on the TDP sensors when continuous heating is used. In order to achieve this, an innovative laboratory setup was designed to replicate the NTGs of WLEs by using temperature-controlled rooms and a heat induction coil wrapped around real tree stems connected to a water flow system. Both thermal systems were controlled by monitoring with an infra-red camera.

\subsubsection{Optimisation of TDP sap flow measurements under semi-arid conditions}

There is no single method that complies with all the requirements needed to obtain accurate, precise, and representative water uptake quantifications. In order to understand the hydrological role of phreatophyte trees in WLEs, such information is of the utmost interest. Therefore, in order to obtain reliable quantifications of water uptake, it is necessary to use a combination of sap flow methodologies to overcome the limitations of each method and to properly cover the natural variability of tree responses assessed by sap flow measurements.

The proposed measuring scheme combining techniques such as the efficient and cost-effective TDP in its CHD implementation and the versatile HFD (Nadezhdina et al., 2002) allowed correction and compensation for the main limitations (e.g. costs, measuring points, number of trees) and biases (e.g. NTG and sap flow variability) of the sap flow methods in one single financially efficient campaign.

This combination of techniques constitutes a crucial step for sap flow measurement techniques under conditions such as those of WLEs (highly contrasting temperatures between night and day, generating biases in the thermal methods used for sap flow measuring). Moreover, it enabled the identification of new traits and characteristics unique to each species, enhancing the understanding of their roles and strategies in WLEs.

\subsubsection{Estimation of tree transpiration at different spatiotemporal scales using remotely sensed imagery}

Tree transpiration functions at different spatiotemporal scales. Small scales are of interest in understanding how the process affects trees and their surroundings (particularly demanded in plant physiology studies). However, in hydrological terms the tree scale is not sufficient to encompass the characteristics necessary to understand the dynamics of transpiration for water management. Thus, quantifications at the level of a forest stand or even a catchment are those most demanded by hydrologists and managers (Lubczynski, 2009).

A reliable alternative to quantifying transpiration at the catchment level is to upscale sap flow measurement using biometric characteristics de- rived from high spatial and spectral resolution remotely sensed imagery (Lubczynski, 2009). Using remotely sensed images for scaling up is advantageous because it enables the high spatial variability of the 
trees to be properly covered, especially when the forest is not composed of many tree species (Lubczynski, 2009), as is common in WLEs.

Besides the initial study of Chavarro-Rincón (2009), there are no studies reported in literature using object attributes of the vegetation to scale up sap flow. Thus this study has pioneered the application of a scaling-up technique based on remote sensing by (1) directly relating remote sensing measurements of projected canopy areas to sapwood areas, (2) better integrating the inner variability of tree sizes, and (3) additionally evaluating its uncertainty.

With such a degree of information, the identified spatiotemporal patterns of the hydrological behaviour of the vegetation provided an advantageous viewpoint to understand how phreatophytic trees survive very dry conditions, how their uptake varies from the beginning to the end of the dry season for both species, and how they might affect the hydrological dynamics in a catchment.

\subsubsection{Sourcing tree transpiration by naturally abundant isotope tracing and tree water flow modelling}

Different studies have provided indications of how sources of water contribute differently to transpiration in trees (Zencich et al., 2002; David et al., 2007), but there is little knowledge about the driving forces causing certain sources to be more intensively used than others. Moreover, such measurements have a limited temporal coverage, due to the technical challenges of measuring naturally abundant isotopes.

This section of the study focused on the hourly provision of isotopes measurements to explain the source contribution based on contrasts in naturally occurring stable hydrogen isotopes in groundwater and unsaturated zone water, thereby addressing objective no. 5. This required very intensive water sampling fieldwork regarding the soil, groundwater, and the sapwood of both Quercus ilex and Quercus pyrenaica.

The gathered information was indispensable for validating the modelling framework and thus understanding the mechanisms controlling the transpiration source contributions at the beginning and at the end of the dry season (points at which the humidity conditions in the subsurface are very different). Furthermore, surprising insights were gained regarding the uptake dynamics of the trees, which followed more the dynamics of a riparian species than those of a woodland species. This was due to the shallow groundwater conditions of the investigated trees in the Sardón catchment.

\subsubsection{Unravelling the spatial variability of tree transpiration water sources}

The discrimination of water sources has become an important focus of hydrological studies of several regions and various hydrological conditions (David et al., 2007; Zencich et al., 2002; Bredemeier et al., 2011). However, to be able to provide practical guidelines, such information is 
most useful when linked to the dynamics of higher organisational scales such as catchments.

This can be achieved provided that an accurate and significantly large data set on water uptake is available, as well as a complementary methodology to extrapolate the source contribution of the subsurface compartments to larger scales. However, such data sets are uncommon and require a lot of resources.

Fortunately, the other sections of this doctoral research provided the necessary breadth and depth of information to combine accurate sap flow measurement, tracing of naturally abundant isotopes, and scalingup methods - hence providing a unique opportunity to understand the spatiotemporal variation of water sources in transpiration ( $T_{g}$ from the groundwater and $T_{u}$ from the unsaturated zone).

The final output provided an intricate description of the spatial dynamics of tree transpiration at the catchment scale, while highlighting the differences and unique properties of Quercus ilex and Quercus pyrenaica.

\subsection{Research objectives}

\subsubsection{Main objectives}

Recognising the active role of the vegetation in WLEs, and its impact upon the hydrological resources, the aims of this research were:

- To define and quantify the tree transpiration and groundwater uptake dynamics of the phreatophytes Quercus ilex Desf. Samp. and Quercus pyrenaica Willd. at the tree, stand and catchment spatial scales.

- To increase the understanding of the water-use dynamics of Quercus ilex and Quercus pyrenaica Willd. at such scales.

\subsubsection{Specific objectives}

To achieve the main objectives of this research, the following specific objectives were addressed to coherently solve the scientific problems stated in Section 1.3:

1. To identify the biasing effect of natural temperature gradients (NTGs) on the performance of the thermal dissipation probe (TDP) method under the conditions prevailing in the semi-arid study area.

2. To test and validate the cyclic heat dissipation (CHD) method (Subsection 1.3.1)

The aim of these objectives was to provide more information about the NTG bias and to test and validate the performance of the CHD method developed at the Department of Water Resources of the Faculty of Geo-information Science and Earth Observation of the University of Twente. 
3. To develop a step-wise optimisation method of TDP sap flux density $\left(J_{p}\right)$ measurements to handle its biases and limitations.

This objective was set to design an accurate and reliable means of quantifying sap flow in semi-arid conditions for Q.i. and Q.p., while minimising the following drawbacks: (a) NTG bias; (b) $\Delta T_{\max }$ bias due to nocturnal flow; (c) radial and azimuthal $J_{p}$ variability. Furthermore, it was expected to obtain new knowledge of the wateruse dynamics of the species at the tree level that could explain their water uptake strategies to survive very dry conditions.

4. To upscale quantifications of transpiration in Q.i. and Q.p. to the whole tree cover within the Sardón catchment and evaluate the robustness and uncertainties of this method (Subsection 1.3.3).

This set of objectives was geared towards describing the methodology to scale up sap flow measurements and calculate the transpiration of forest stands and entire catchments, and to obtain ecohydrological insights on Q.i. and Q.p. at higher organisational scales. Additionally it was aimed at calculating the uncertainty of the scaling-up exercise.

5. To determine the source contributions of the groundwater and the unsaturated zone of the soil to the transpiration of $Q . i$. and Q.p. trees using stable isotope tracing experiments.

6. To explain the obtained source contributions through the singletree stem diameter flow model of Steppe et al. (2006).

With these objectives in mind, it was planned to determine the contribution of diverse water sources to transpiration for the studied phreatophyte species (i.e. Q.i. and Q.p.), as well as to explain how such partitioning is governed by the water potentials that the trees are exposed to. Additional insights on the species' strategies to uptake water are expected to be revealed.

7. To identify the spatial variation in the water contributions to tree transpiration at the catchment scale, as dependent on the Q.i. and $Q . p$. vegetation cover.

The last stage of this research focused on demonstrating how the results and insights of previously described methodologies are put together to illustrate the spatial variability of the tree transpiration water source components across the whole Sardón catchment. Additionally, traits and characteristics of Q.i. and Q.p. were to be highlighted in the context of water management at the catchment level.

\subsection{Thesis outline}

To quantify the groundwater component of the transpiration and to understand its hydro-physiological dynamics in time and space, one laboratory experiment, two dry-season campaigns of sap flow monitoring, 
and four outdoor experiments were conducted to gather the necessary data.

In Chapter 2 the laboratory experimentation of artificial thermal gradients on stem segments and the gravimetric validation of the CHD method are illustrated, addressing specific objectives 1 and 2. This validation was necessary to ensure the accuracy of the sap flow monitoring when using the TDP sensors in follow-up campaigns. The CHD methodology was used as the pillar of the step-wise field measurements.

Chapter 3 presents the field optimisation methodology of sap flow measurements and the sap flow information collected at the tree level, addressing specific objective 3 . Important attention was dedicated to the development of the step-wise optimisation method, because the sap flow data were the main input for the scaling-up method described in the chapter that follows.

Chapter 4 illustrates the scaling up of sap flow measurements to tree transpiration across stand and catchment scales, using object attributes derived from high-resolution satellite imagery, hence addressing specific objective 4 . The method was applied to the Sardón catchment located in Salamanca Province, Spain. Additionally, the chapter describes how to fill in data gaps in sap flow measurements, as well as an uncertainty analysis of the scaling-up procedure from remote sensing data.

Chapter 5 elaborates on the methodological and scientific aspects necessary to obtain the quantification of the tree transpiration water source components, addressing specific objective 5 . In this chapter a whole-tree water flow model was used to explain the contributions found with the naturally abundant isotopes, addressing specific objective 6 .

Chapter 6 contains the integration of the inputs from all the previous chapters by scaling up the source components of the tree transpiration, namely: the water contributions of the (a) saturated and (b) unsaturated zones of the soil, thus addressing specific objective 7 . The scaling-up described in this chapter focused on the beginning of the dry season (i.e. June), when Q.p. had developed leaves, and on the end of the dry season (i.e. August-September), when the lowest values of soil moisture were obtained. The identified spatial patterns are analysed and their ecohydrological implications discussed. 


\section{Correction of natural temperature gradients in sap flow measurements}

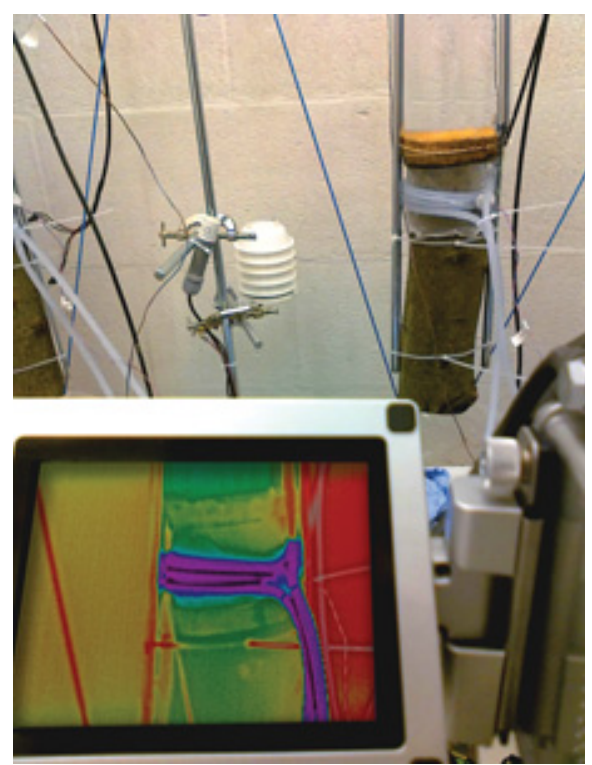

Figure 2.1: Gravimetric water flow tests monitored by infrared imagery.

This Chapter is based on: Novel, cyclic heat dissipation method for the correction of natural temperature gradients in sap flow measurements. Part 2. Laboratory validation. ReyesAcosta, J.L., M.W. Vandegehuchte, K. Steppe and M.W. Lubczynski. (2012). Published in Tree Physiology. 


\begin{abstract}
Sap flow measurements conducted with thermal dissipation probes (TDPs) are vulnerable to natural temperature gradient (NTG) bias. Few studies, however, attempted to explain the dynamics underlying the NTG formation and its influence on the sensors' signal. This study focused on understanding how the TDP signals are affected by negative and positive temperature influences from NTG and tested the novel cyclic heat dissipation (CHD) method to filter out the NTG bias. A series of three experiments were performed in which gravity-driven water flow was enforced on freshly cut stem segments of Fagus sylvatica L., while an artificial temperature gradient (ATG) was induced. The first experiment sought to confirm the incidence of the ATG on sensors. The second experiment established the misestimations caused by the biasing effect of the ATG on standard TDP measurements. The third experiment tested the accuracy of the CHD method to account for the ATG biasing effect, as compared with other cyclic correction methods. During experiments, sap flow measured by TDP was assessed against gravimetric measurements. The results show that negative and positive ATGs were comparable in pattern but substantially larger than field NTGs. Second, the ATG bias caused an overestimation of the standard TDP sap flux density of $\approx 17 \mathrm{~cm}^{3} \mathrm{~cm}^{-2}$ hour $^{-1}$ by $76 \%$, and the sap flux density of $\approx 2 \mathrm{~cm}^{3} \mathrm{~cm}^{-2}$ hour $^{-1}$ by over $800 \%$. Finally, the proposed CHD method successfully reduced the max. ATG bias to $25 \%$ at $\approx 11 \mathrm{~cm}^{3} \mathrm{~cm}^{-2}$ hour ${ }^{-1}$ and to $40 \%$ at $\approx 1 \mathrm{~cm}^{3} \mathrm{~cm}^{-2}$ hour $^{-1}$. We concluded that: (i) the TDP method is susceptible to NTG especially at low flows; (ii) the CHD method successfully corrected the TDP signal and resulted in generally more accurate sap flux density estimates (mean absolute percentage error ranging between 11 and 21\%) than standard constant power TDP method and other cyclic power methods; and (iii) the ATG enforcing system is a suitable way of re-creating NTG for future tests.
\end{abstract}

\title{
2.1 Introduction
}

Thermal methods for measuring sap flow are widely used in hydrology, tree physiology, ecology and other fields to quantify and better understand the water use dynamics in the plant-atmosphere-soil continuum (Marshall, 1958; Swanson, 1994; Lubczynski, 2000; Burgess et al., 2001; Nadezhdina et al., 2002; Lubczynski and Gurwin, 2005; David et al., 2007; Dragoni et al., 2009). Such understanding is often related with achieving an accurate and precise estimation of whole-plant water use and how such water consumption can influence the environment (Williams et al., 2004; Hernández-Santana et al., 2008a; Lubczynski, 2009). In recent years, several studies have analysed the hydrological role of vegetation and its water use based on up-scaled measurements (Granier, 1996; Wilson et al., 2001; Williams et al., 2004; Cavanaugh et al., 2011; Miller et al., 2010; Yaseef et al., 2010). However, many of such studies have not put enough attention to evaluate the accuracy of sap flow measurements 
and errors or biases that influence them. This is also the case when using the thermal dissipation probe (TDP) method of Granier $(1985,1987)$, one of the most widely used sap flow measuring methodologies, thanks to its low cost and ease of use. Nevertheless, the TDP has a series of drawbacks that, if not accounted for, can generate a large error while estimating plant water use (Do and Rocheteau, 2002b; Lu et al., 2004; Steppe et al., 2010). Most of these drawbacks can be solved or minimized (Lu et al., 2004), but when the TDP method is used in semi-arid open forests or orchards, the bias caused by natural temperature gradients (NTGs) can be significant and difficult to deal with (Braun and Schmid, 1999; Do and Rocheteau, 2002a; Lubczynski et al., 2012), sometimes even creating errors larger than 100\% (Do and Rocheteau, 2002b; Reyes-Acosta and Lubczynski, 2014).

The influence of NTG biases have been attributed to uneven temperature in the sapwood that affects one or both probes of the TDP sensor (Čermák and Kučera, 1981; Lundblad et al., 2001; Do and Rocheteau, 2002a,b; Lu et al., 2004; Tatarinov et al., 2005). Several authors Čermák and Kučera (1981); Köstner et al. (1998); Braun and Schmid (1999); Do and Rocheteau (2002a) attributed the NTG to differences in heat storage in the soil, stem and root tissues resulting in the lower temperature of the sap flowing from the roots early in the morning, as compared with the stem. Lu et al. (2004) demonstrated the role of direct solar radiation onto the stem as an additional source of NTG. Besides these explanations, there is no clear understanding of the exact dynamics generating the NTG influence. Field measurements using unpowered TDPs, to measure the temperature difference between probes installed $10 \mathrm{~cm}$ apart, have demonstrated that the NTG can be significant for several species and ranges between the following values: from -2.00 to $+1.46{ }^{\circ} \mathrm{C}$ in Acacia tortilis (Forssk.) Hayne (Do and Rocheteau, 2002a), from -2.45 to +1.38 ${ }^{\circ} \mathrm{C}$ in Quercus ilex ssp. ballota L. (Desf.) Samp, from -0.49 to $+1.00{ }^{\circ} \mathrm{C}$ in Quercus pyrenaica Willd. (Reyes-Acosta and Lubczynski, 2014), from -1.18 to $+0.14{ }^{\circ} \mathrm{C}$ in Boscia albitrunca (Burch.) Gilg \& Ben., from -5.20 to $+0.97{ }^{\circ} \mathrm{C}$ in Dichrostachys Wight et Arn., from -1.65 to $0.98{ }^{\circ} \mathrm{C}$ in Acacia fleckii Schinz and from -1.69 to $0.68{ }^{\circ} \mathrm{C}$ in Acacia luederitzii Engl. (Lubczynski et al., 2012).

Different methods have been proposed to avoid and/or correct the NTG bias, such as: (i) partial-to-full coverage of stem and superficial roots applying reflecting and isolating material (Braun and Schmid, 1999; Lu et al., 2004); (ii) simultaneous monitoring of the variability of the NTG on neighbouring trees of the same species and similar size, to account for the NTG bias in the sap flow estimations (Köstner et al., 1998); (iii) measuring the NTG with another set of probes close to the TDP installation and subtracting its influence (Čermák and Kučera, 1981); (iv) use of a new design of TDPs including an "extra" pair of thermocouples (Goulden and Field, 1994; Nourtier et al., 2011); and (v) use of a cyclic power schema (CPS), i.e., switching repeatedly power ON and OFF, to account for the variability of the NTG patterns on the sap flow measurements (Köstner et al., 1998; Do and Rocheteau, 2002a,b; Isarangkool Na Ayutthaya et al., 
2010; Lubczynski et al., 2012).

The selection of the method to account for the NTG bias depends on the type of species, study objective, environmental conditions and capacity of the project carried out. However, when the NTG influence is large and a good accuracy and precision is needed, Lu et al. (2004) advised to use a CPS. They highlighted the advantages of cyclic power methodologies, in particular the transient thermal dissipation (TTD) method proposed by Do and Rocheteau (2002b). The TTD uses the same type of probes as designed by Granier but data are measured in short cycles (here references as transients), a signal rising part corresponding with the power $\mathrm{ON}$ (ON-transient) and a signal declining part corresponding with power OFF (OFF-transient). These transients are not long enough to reach thermal equilibrium as is required by the Granier method (Granier, 1985), thus another empirical formula was developed and tested (Do and Rocheteau, 2002a,b). The TTD method has been validated on synthetic porous medium as to be insensitive to stable natural temperature gradients under laboratory conditions (Do and Rocheteau, 2002b). Additionally, validation of the impact of an artificial temperature gradient (ATG) created by inserting an extra pair of heating probes upon the TDP signal was carried out (Do and Rocheteau, 2002b). However, such a way of creating an ATG generates a disturbance of the wood matrix that might affect heat dissipation. Later, Isarangkool Na Ayutthaya et al. (2010) developed an improved multispecies calibration for the TTD method. This calibration was derived from tests in three species: Citrus maxima (Burm.) Merr., Hevea brasiliensis (Willd. ex Adr de Juss.) Muell. et Arg. and Mangifera indica L. The tests concluded that the new multispecies calibration covered the variability of sap flux densities better than the calibration obtained in 2002, and thus it was species independent. These findings provided more confidence into the TTD method. However, the multispecies calibration of the TTD method is not as extensively tested as the original Granier calibration (1985) and it is not clear if it was tested for NTG.

The cyclic heat dissipation (CHD) method for correcting the NTG bias of the TDP signal as presented by Lubczynski et al. (2012) in the companion paper constitutes an improved approach as compared with the TTD method because it: (i) provides physically based retrieval of the extrapolated signal to the steady-state required in the Granier method, which means it benefits from the widely tested Granier formula calibrated on many species by many authors; (ii) allows for a more reliable definition of $\Delta T_{\text {max }}$; (iii) allows a better understanding on how the CPS can be used to correct the NTG bias in the signal. Even though this correction has been tested under field conditions (Lubczynski et al., 2012; Reyes-Acosta and Lubczynski, 2014), a laboratory validation under controlled conditions is required to assess its reliability, accuracy, performance and precision.

Thus, the objectives of this study are: (i) to design and evaluate a heating-cooling system to realistically create ATGs comparable to the NTGs observed under field conditions and regulate its strength without affecting the wood tissue; (ii) to understand the influence of variable 
ATG upon the TDP signal under dissimilar sap flux densities and (iii) to validate sap flow estimations using the CHD correction method under varying ATG conditions and contrasting sap flux densities applying the Mariotte-based verification system of Steppe et al. (2010).

\subsection{Materials and methods}

\subsubsection{Collection and processing of plant material}

The plant material was collected from two 10-20-year-old European beech trees, Fagus sylvatica L., with stems of diameter at breast height (DBH) of $\approx 8 \mathrm{~cm}$ located at the experimental forest "Aelmoeseneie" in Gontrode, Belgium. In order to minimize evaporation from the stem tissues, the cut ends of the segments were covered with plastic bags and sealed with duct tape. The segments were transported immediately to the Laboratory of Plant Ecology, Ghent University, where they were stored and re-cut into smaller pieces. A summary of the plant material used during these tests can be seen in Table 1. The stem segments were post-processed at the end of each experiment to calculate sapwood depth and to verify the homogeneity of the sapwood area. All stem segments were $\approx 30 \mathrm{~cm}$ long except for the one used in Test 1 that was $\approx 20 \mathrm{~cm}$ and the sapwood depth was between 2 and $2.6 \mathrm{~cm}$ with the exception of one segment that had $3.6 \mathrm{~cm}$.

Table 2.1: Biometric measurements of the stem segments of European beech (F. sylvatica) used in the tests

\begin{tabular}{lllll}
\hline & $\begin{array}{l}\text { Length } \\
(\mathrm{cm})\end{array}$ & $\begin{array}{l}\text { Stem } \\
\text { diameter } \\
(\mathrm{cm})\end{array}$ & $\begin{array}{l}\text { Sapwood } \\
\text { area at } \\
\text { reference } \\
\text { needle }\left(\mathrm{cm}^{2}\right)\end{array}$ & $\begin{array}{l}\text { Sapwood } \\
\text { depth at } \\
\text { reference } \\
\text { needle }\left(\mathrm{cm}^{2}\right)\end{array}$ \\
\hline Test 1 & 21.40 & 7.69 & N/A & N/A \\
$\begin{array}{l}\text { Test 2 } \\
\text { Segment 1 }\end{array}$ & 28.50 & 8.91 & 50.24 & 2.5 \\
$\begin{array}{l}\text { Test 2 } \\
\text { Segment 2 }\end{array}$ & 37.40 & 9.33 & 56.82 & 2.6 \\
$\begin{array}{l}\text { Test 3 } \\
\text { Segment 1 }\end{array}$ & 27.20 & 8.91 & 46.66 & 2.0 \\
$\begin{array}{l}\text { Test 3 } \\
\text { Segment 2 }\end{array}$ & 33.30 & 7.92 & 48.97 & 3.6 \\
\hline
\end{tabular}




\subsubsection{Mariotte-based verification system}

To provide a continuous, controlled water flow in the CHD validation experiment, the gravity-driven Mariotte-based verification system was used to enforce water flow in the collected stem segments, as proposed by Steppe et al. (2010) (Figure 2.2). This system consists of a Mariotte's bottle suitable to maintain a constant head of water (McCarthy, 1934) during an experiment. The system was built using a $5 \mathrm{l}$ Erlenmeyer flask and two glass tubes placed at the same depth in the flask. One of the glass tubes worked as an air inlet and the second one was connected through plastic tubing to a third one. The third tube acted as a siphon inside a plastic column filled with water and attached to the stem segments. The Erlenmeyer flask was placed on two platform jacks, so the water flow could be controlled by adjusting the height of the flask and, hence the bottom of the air inlet. The augmented pressure then delivered the necessary amount of water to the water column in the stem segment through the siphon tube and maintained equilibrium at the required water head $h$ ( $h=$ distance between the meniscus in the air inlet and the surface of the stem segment). The enforced water flow was continuously measured gravimetrically using an electronic balance (PS 4500/C1, Henk Maas Weegschalen BV, Veen, the Netherlands).

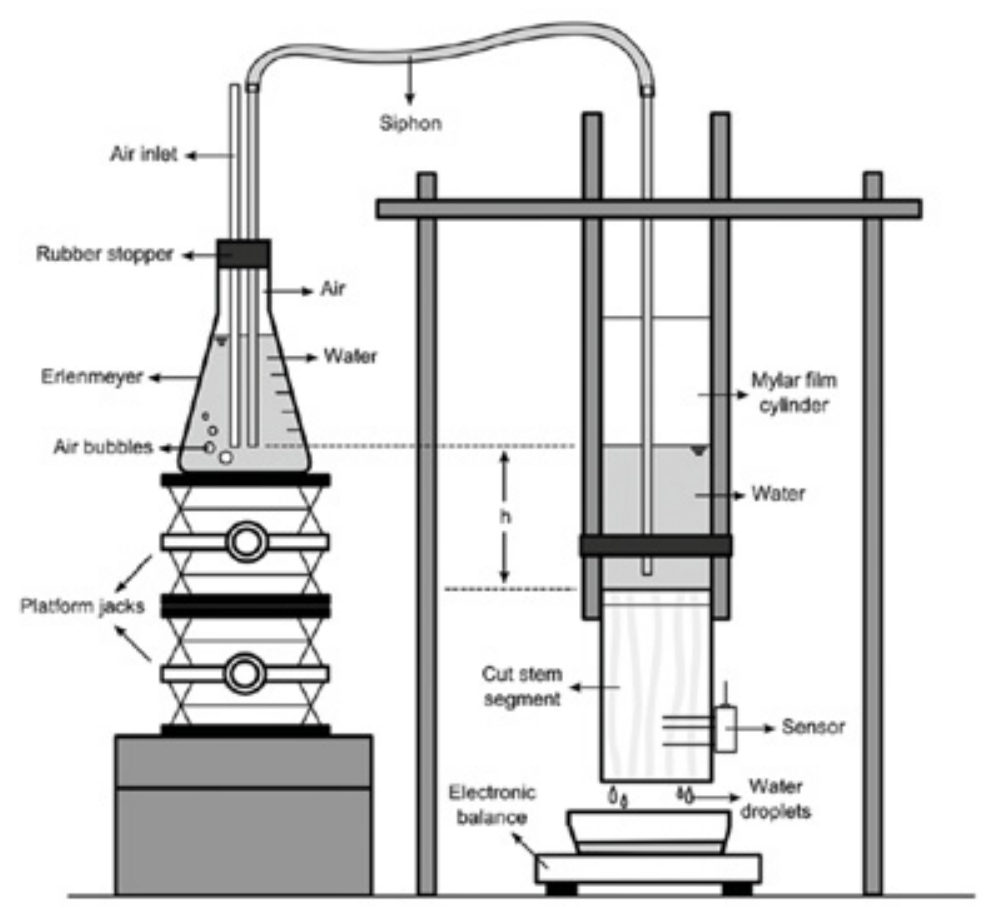

Figure 2.2: Diagram of the Mariotte water flow verification system (from Steppe et al. (2010)) used in all experimental set-ups 
For a proper functioning of the water flow system, the stem segments required some processing in order to assure a continuous unbiased flow. First, the cut surfaces were rewetted and visually examined (using a stereo microscope) to find eventually clogged sapwood vessels. If such vessels were found, the wood surface was trimmed using a scalpel until the vessels were completely opened. Second, a $2 \mathrm{~cm}$ wide bark-strip was removed from the upper side of the stem segments to firmly attach a plastic column directly on the sapwood. This assured that water was only conducted through the xylem. The plastic column was fixed with a double-sided adhesive tape and a thick silicone layer. Two stem segments were prepared $\approx 18 \mathrm{~h}$ before each experimental phase, time enough for the silicone paste to harden properly. These and more details are given in Steppe et al. (2010).

\subsubsection{Artificial thermal gradient enforcement}

In order to induce ATGs in the stem segments, an experimental ATG enforcement system was designed to expose the segments to two contrasting temperatures: (i) air temperature and (ii) temperature enforced in the topside of the stem. The ATG enforcement system consists of three parts: (i) a temperature regulated water circulation system, adjusting stem temperature; (ii) air temperature regulation; and (iii) a thermal infrared camera to monitor and verify the formation of the ATG on the segments (NEC, Thermal Imager R300 PANORAMA, Tokyo, Japan).

The temperature-regulated water circulation system is a flexible plastic tubing of $1 \mathrm{~cm}$ diameter and $\approx 2 \mathrm{~m}$ length connected to a water thermostatic bath (20L GRANT GD120,Cambridge, UK) with a cooling unit (GRANT C1G, Cambridge, UK). The tubing transports water kept by the bath at a constant temperature, at the following temperature range: $0-36{ }^{\circ} \mathrm{C}$. The tubing was wrapped around the upper side of the stem to mimic the heat effect observed during the NTG formation on trees in the field (i.e., differences of storage and thermal resistance of the soil-wood continuum). The air temperature was regulated by an air-conditioning system inside a growth room, where the experiments took place. The room was $\approx 5 \mathrm{~m}^{2}$ and provided the possibility of swiftly changing the air temperature from 15 to $30{ }^{\circ} \mathrm{C}$, a temperature range similar to night and day air temperatures in semi-arid regions.

The ATG enforcement system was operated in two contrasting modes simulating the most critical NTG field conditions, early in the morning and after sunset. In Mode I for simulating NTG after sunset, the circulating water from the top representing stem temperature was set at $36{ }^{\circ} \mathrm{C}$ and the air temperature at $15,{ }^{\circ} \mathrm{C}$ as observed in semi-arid open forests in Salamanca, Spain (Reyes-Acosta and Lubczynski, 2014). In Mode II simulating early morning NTG, the circulating water temperature was set at $10 a^{\circ} \mathrm{C}$ nd the air temperature at $29 .{ }^{\circ} \mathrm{C}$ The formation of the temperature gradients was monitored with the thermal camera by taking images every 10 minutes (Figure 2.3). 


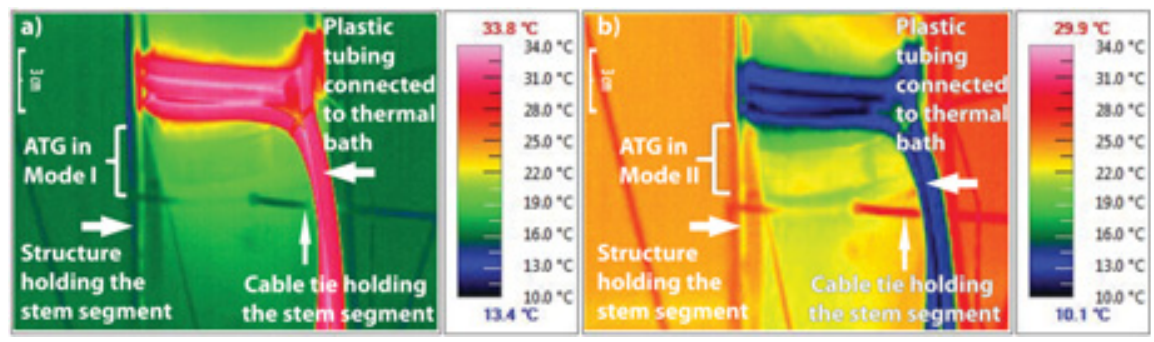

Figure 2.3: Infrared images monitoring the ATGs produced by the ATG enforcement system on the stem segments during the experiments: (a) shows the stem segments under Mode I and (b) under Mode II. The TDP reference needle is $\approx 10 \mathrm{~cm}$ below the tubing and the heating probe another $10 \mathrm{~cm}$ below. The TDP sensors were intentionally left out of the images (they are in the backside of the stem), to avoid interferences with the observation of the temperature gradients.

\subsubsection{Thermal dissipation probes for measuring sap flux densities}

We monitored the sap flux density passing through the stem segments in each of the experiments using $20 \mathrm{~mm}$ long, standard Granier-type (Granier, 1985) TDP sensors (UP Gmbh, Germany). The TDPs were installed radially in the stems, $10 \mathrm{~cm}$ apart and $2 \mathrm{~cm}$ deep, with the heating probe placed at the downstream water flow position, i.e., below the non-heated probe.

The TDP method uses an empirical calibration, which relates the temperature difference between the unheated and heated probe $(\Delta T$ in ${ }^{\circ} \mathrm{C}$ ) to the sap flux density $\left(J_{p}\right.$ in $\mathrm{cm}^{3} \mathrm{~cm}^{-2}$ hour $\left.{ }^{-1}\right)$, as in Eq. (2.1 and 2.2) (Granier, 1985)

$$
\begin{aligned}
& J_{p}=0.119 K_{a}{ }^{1.231} \times 3600 \\
& K_{a}=\frac{\Delta T_{\max }-\Delta T}{\Delta T}
\end{aligned}
$$

where $\Delta T_{\max }$ is a reference temperature difference when $J_{p}$ is equal to zero, i.e., at no flow conditions. In this study the $\Delta T$ measurements for standard TDP (with constant power) were recorded every $30 \mathrm{~s}$ and stored at 10 min intervals in a Skye Datahog2 logger while $\Delta T_{\max }$ was determined at the end of each experiment at no-flow condition, by measuring $\Delta T$ until its stabilization was achieved.

\subsubsection{Cyclic power scheme for correcting the influence of ATG in TDP}

The CHD method was applied using a cyclic power scheme (CPS) of $\Delta T$ data acquisition, as suggested by Lubczynski et al. (2012). The CPS 
consisted of 30 seconds resolution measurements in consecutive cycle transients of $7.5 \mathrm{~min}$ power ON and $7.5 \mathrm{~min}$ power OFF, resulting in four cycles per hour. This time set for the ON-OFF transients was defined by optimization of the minimum number of samples per interval, still allowing an adequate extrapolation of the signal to asymptotic thermal equilibrium (Figure 2.4). The alternated ON-OFF cycles were controlled using a custom-specific, programmable power-relay regulator designed to count switching power intervals controlled by the logger.

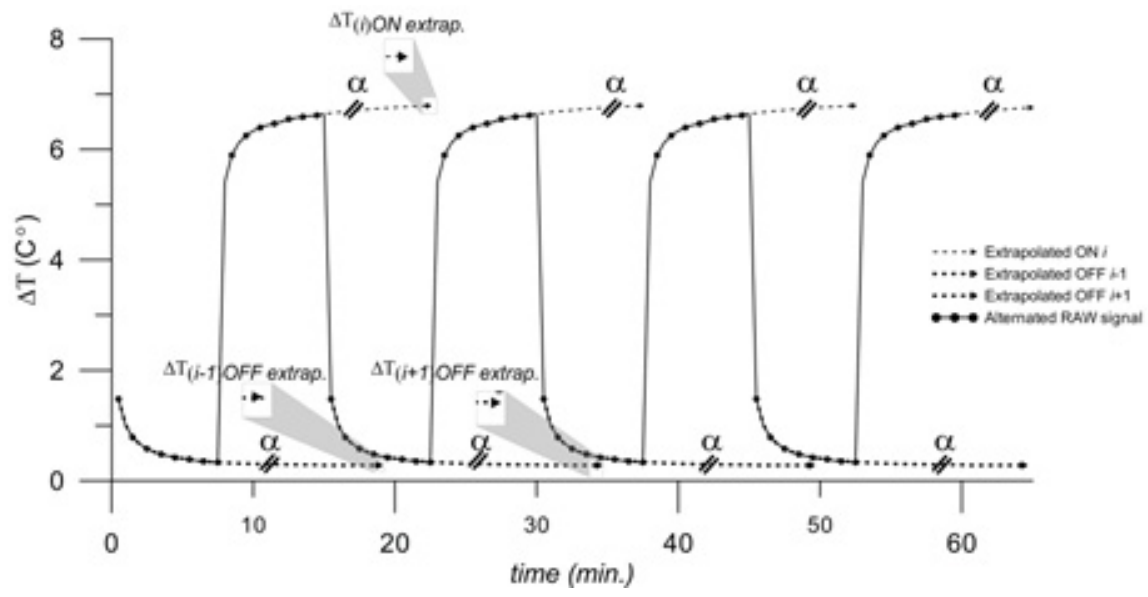

Figure 2.4: Thermal dissipation probe alternated-power cycle (bold line) with its corresponding extrapolated ad-infinitum transients ON/OFF (dotted lines). Zoomed areas emphasize the position of the variables needed for Eqs. (2.3- (2.7) in the NTG-bias correction.

The principle of the CHD method Lubczynski et al. (2012) is to subtract the NTG bias $\left(\Delta T_{N T G_{i}}^{E}\right)$ from the extrapolated, to steady-state, power ON signal $\left(\Delta T_{O N_{i}}^{E}\right)$ (Eq. 2.3).

$$
\begin{aligned}
& \Delta T_{O N_{i}}^{C}=\Delta T_{O N_{i}}^{E}-\Delta T_{N T G_{i}}^{E} \\
& \Delta T_{N T G_{i}}^{E}=0.5\left(\Delta T_{O F F_{i-1}}^{E}+\Delta T_{O F F_{i+1}}^{E}\right)
\end{aligned}
$$

In Eq. (2.3), $\Delta T_{O N_{i}}^{C}$ is an NTG-corrected $\Delta T$ (in this case ATG-corrected) in the $i$-th transient, $\Delta T_{N T G_{i}}^{E}$ is the temperature bias caused by the NTG influence and $\Delta T_{O N_{i}}^{E}$ is $i$-th cycle power ON signal extrapolated to steady state. In Eq. (2.4), $\Delta T_{N T G_{i}}^{E}$ is calculated as as an average of the two extrapolated to steady-state OFF-transients, the preceding $\left(\Delta T_{O F F_{i-1}}^{E}\right)$ and succeeding $\left(\Delta T_{O F F_{i+1}}^{E}\right)$ (Figure 2.4). The extrapolated to steady-state , $\Delta T_{O N_{i}}^{E}, \Delta T_{O F F_{i-1}}^{E}$ and $\Delta T_{O F F_{i+1}}^{E}$ signals are calculated using Eqs. (2.5), (2.6) and (2.7), respectively, following Lubczynski et al. (2012)

$$
\Delta T_{O N_{i}}^{E}=\Delta T_{O F F_{i-1}}^{E}+A_{1}\left(1-e^{-t / T_{1}}\right)+A_{2}\left(1-e^{-t / T_{2}}\right)+A_{3}\left(1-e^{-t / T_{3}}\right)
$$




$$
\begin{gathered}
\Delta T_{O F F_{i-1}}^{E}=\Delta T_{O N_{i-2}}-\left[A_{1}\left(1-e^{-t / T_{1}}\right)+A_{2}\left(1-e^{-t / T_{2}}\right)+A_{3}\left(1-e^{-t / T_{3}}\right)\right] \\
\Delta T_{O F F_{i+1}}^{E}=\Delta T_{O N_{i}}-\left[A_{1}\left(1-e^{-t / T_{1}}\right)+A_{2}\left(1-e^{-t / T_{2}}\right)+A_{3}\left(1-e^{-t / T_{3}}\right)\right]
\end{gathered}
$$

In Eqs. (2.5), (2.6) and (2.7) the time $t=\infty$ and $A_{1}, A_{2}, A_{3}$ are parameters defining amplitudes of the following thermal components: (i) the heater filament and all the probe components; (ii) the aluminium tubes and silicon compound (inserted in the sapwood during installation of the probe); and (iii) the sapwood tissue. $T_{1}, T_{2}$ and $T_{3}$ are time constants defining the operating time of each thermal component. Fitting the amplitude and time parameters for the model to extrapolate the raw signals of each cycle (Eqs. (2.3), (2.5) and (2.6)) is a complex and timeconsuming procedure. Thus, an automated MATLAB routine was used for: (i) fitting the signal by optimizing $A_{i}$ and $T_{i}$; (ii) calculating the NTG correction; and (iii) $J_{p}$ calculation. In the optimization process $T_{1}$ and $T_{2}$ were set as time-independent constants as they refer to non-variable circuits (i.e., heat filament, probes and aluminium tube), as proposed by Lubczynski et al. (2012). However, parameters $T_{3}, A_{1}, A_{2}$ and $A_{3}$ were optimized for each transient, according to Eqs. (2.3) and (2.4), respectively.

\subsubsection{Experimental design}

The experiments were developed under laboratory conditions to: (i) provide a controlled environment in which it was possible to regulate the flowing water in fresh stem samples; and (ii) control external sources of heat affecting air and stem temperature to mimic NTG dynamics resulting from the contrasting and temporally variable day/night temperature variability. The water used for all tests was deionized and stored inside the growth room to maintain the same temperatures of the other elements and hence optimal for its use.

\subsubsection{Test 1: ATG formation monitoring}

The first test was designed to assess whether the ATG enforcement system was suitable to create temperature gradients comparable to those affecting the TDP signal in field conditions. Thus, the objective was to create, regulate and control the ATG simulating NTG. To monitor the ATG, an unpowered TDP sensor was installed in the stem segment exposed to the Mariotte-based verification system (Figure 2.2), set on zero water flow. The test started at 16:30 in Mode I (air temperature at $15{ }^{\circ} \mathrm{C}$ and circulating water temperature at $36{ }^{\circ} \mathrm{C}$ ) and continued until 9:00 the next day when Mode II (air temperature at $29^{\circ} \mathrm{C}$ and circulating water temperature at $10{ }^{\circ} \mathrm{C}$ ) was started and lasted until 18:00 (Figure 2.5). The obtained $\Delta T$ measurements were assessed according to the 
threshold value for nonnegligible temperature gradients proposed by (Do and Rocheteau, 2002a). They proposed, as a first approximation, that if $\Delta T$ values are $>0.2{ }^{\circ} \mathrm{C}$ or $<-0.2{ }^{\circ} \mathrm{C}$, the impact of NTG upon the TDP signal generates a non-negligible $\geq 10 \%$ error in sap flow rate. Thus, $\Delta T$ measurements crossing that threshold were considered as an indication of significant ATG influence upon the TDP signal.

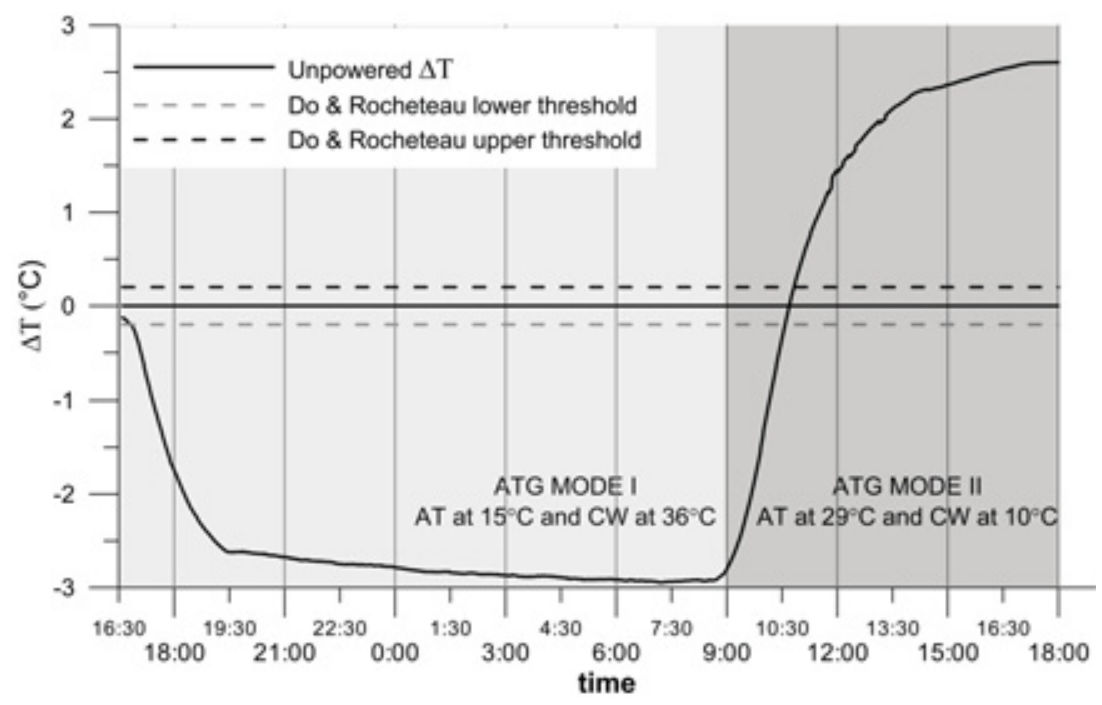

Figure 2.5: Test 1: monitoring the ATG with un-powered TDP signal at zero flow. AT, air temperature; CW, circulating water temperature.

\subsubsection{Test 2: ATG effect upon the standard TDP signal and water flow estimations}

In the second test, the effect of ATG on the standard TDP signal and the sap flux density estimation was monitored and compared with the gravimetric sap flux density. Two stem segments with plastic columns were used and one set of TDP sensors with continuous power supply was installed in each stem segment as described before. Then, each stem column was filled with $\approx 7 \mathrm{~cm}^{3}$ of water to verify that it was flowing through the stem segments and that there were no leaks in the columns. The obtained sap flux densities were different in each segment due to their unique wood-tissue characteristics, even with the same water levels in the column. As a final step, the plastic tubing from the ATG enforcement system was wrapped around the segments. At the beginning of the test, from 11:30 to 13:15 h, the ATG enforcement system was at the same temperature as the room, i.e., without ATG formation, to guarantee that the temperature from the TDP signal and the flowing water achieved thermal equilibrium. This period was called "stabilization". At 13:15 h, the ATG enforcement system was switched to Mode I (air temperature at $15 a^{\circ} \mathrm{C}$ nd circulating water temperature at 36$)^{\circ} \mathrm{C}$ and then after $2 \mathrm{~h}$ and 
$15 \mathrm{~min}$ (at 15:30 h) to Mode II (air temperature at $29 a^{\circ} \mathrm{C}$ nd circulating water temperature at 10$)^{\circ} \mathrm{C}$ and let run for $2 \mathrm{~h}$ and $30 \mathrm{~min}$, until 18:00 h (Figure 2.6).
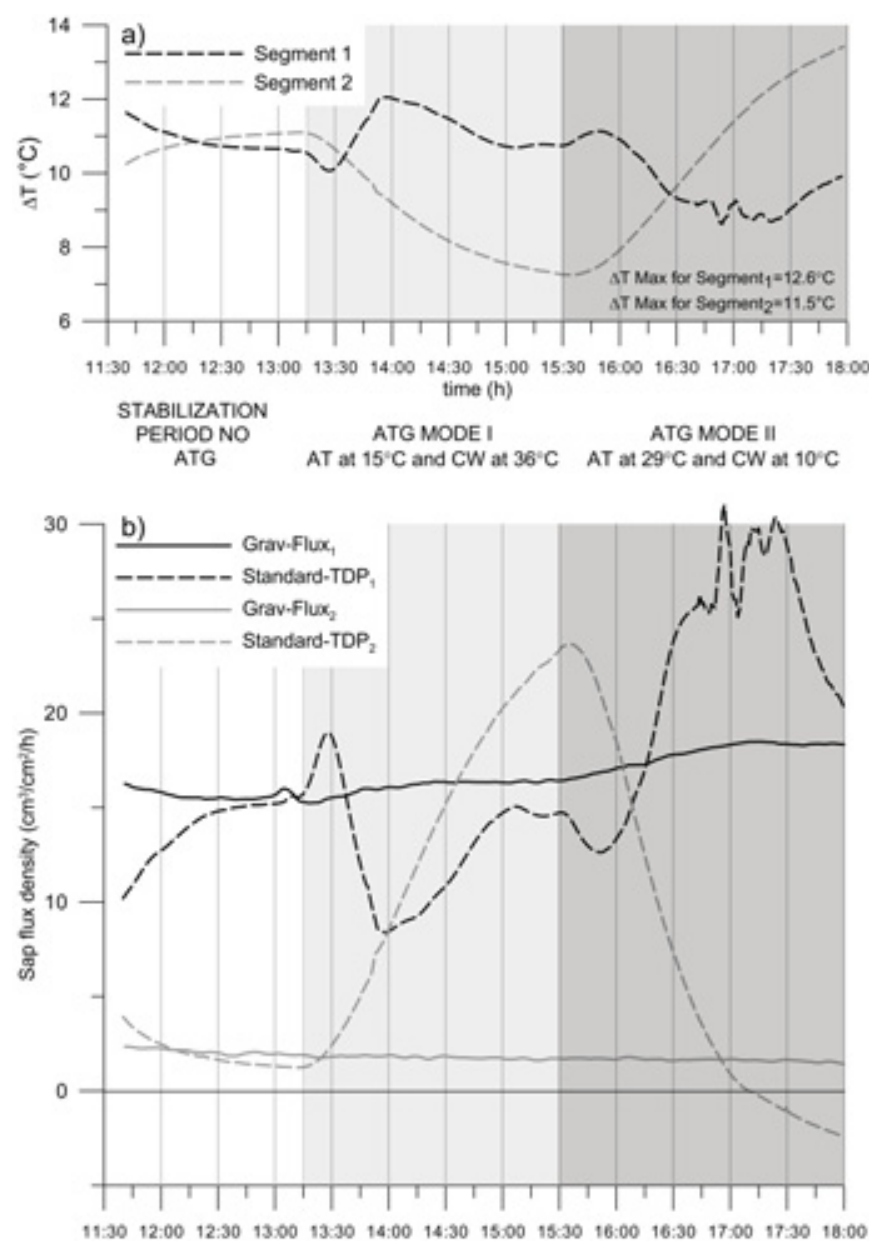

Figure 2.6: Test 2: impact of ATG upon the Standard-TDP measurements for two stem segments with different sap flux densities (Segment 1 with $J_{p} \approx 17 \mathrm{~cm}^{3} \mathrm{~cm}^{-2}$ hour ${ }^{-1}$ and Segment 2 with $J_{p} \approx 2 \mathrm{~cm}^{3} \mathrm{~cm}^{-2}$ hour $^{-1}$ ): (a) raw TDP measurements of $\Delta T$ for both stem segments; (b) comparison of Standard-TDP $J_{p}$ vs. gravimetric control (Grav-Flux). AT, air temperature; $\mathrm{CW}$, circulating water temperature.

The sapwood area of both segments was measured by adding a dye (commercial food colourant) to the water column following Steppe et al. (2010). The chemical was let run for $>7 \mathrm{~h}$ to properly stain the whole sapwood area of the segment. Several discs were cut after staining and verified that the installations of the TDP sensors properly covered the sapwood tissue $(\approx 2 \mathrm{~cm}$ depth$)$ and were not in contact with the 
heartwood. Additionally, to guarantee low circumferential $J_{p}$ variability in the selected segments, microscopic investigations of the uniformity of the colouring dye across the conductive sapwood area were carried out in the cut discs. If a segment presented a non-uniform dying pattern of sapwood area, the results were considered as uncertain and excluded from further analysis. This procedure followed the findings of Čermák et al. (2000) and Saveyn et al. (2008), who pointed out the relation between the uniformity of the conductive sapwood structure and low circumferential $J_{p}$ variability.

The measured sapwood areas were used to convert the gravitational flow into gravitational flux densities (Grav-Flux in Figure 2.6) in order to compare them with $J_{p}$ obtained with standard TDP (presented as Standard-TDP in Figure 2.6). The gravitational flux densities followed low (0.5-2 $\mathrm{cm}^{3} \mathrm{~cm}^{-2}$ hour $\left.{ }^{-1}\right)$ and mean values (11-14 $\mathrm{cm}^{3} \mathrm{~cm}^{-2}$ hour $\left.{ }^{-1}\right)$ of the $J_{p}$ range observed in field conditions for F. sylvatica. This range corresponds to the lower $J_{p}$ value range for the species, ensuring low radial variability (Steppe et al., 2010), and low circumferential variability (Saveyn et al., 2008).

\subsubsection{Test 3: Validation of the CHD method for NTG correction}

The third test was designed to validate the efficiency of the CHD method to correct CPS TDP measurements biased by ATG. This was done by comparing the sap flux density estimations obtained from the CHD method with the gravimetric sap flux densities.

Following the same procedure as in Tests 1 and 2, new stem segments were prepared and one set of TDP sensors installed in each segment. During this test, the two segments produced different sap flux densities $\left(11 \mathrm{~cm}^{3} \mathrm{~cm}^{-2}\right.$ hour ${ }^{-1}$ for segment 1 and $1 \mathrm{~cm}^{3} \mathrm{~cm}^{-2}$ hour $^{-1}$ for segment 2), despite the use of same water levels in the columns (Figure 2.7), as in Test 2. The test started at 12:30, and first the system was stabilized for $30 \mathrm{~min}$. At 13:30 the ATG enforcement system was set in Mode I (air temperature at $15 a^{\circ} \mathrm{C}$ nd circulating water temperature at 36$)^{\circ} \mathrm{C}$, and run for $2 \mathrm{~h}$ and $15 \mathrm{~min}$. After this time period the ATG enforcement system was set in Mode II (air temperature at $29 a^{\circ} \mathrm{C}$ nd circulating water temperature at 10$)^{\circ} \mathrm{C}$, and let run for $2 \mathrm{~h}$ and $15 \mathrm{~min}$. During the whole test, one set of TDP sensors per segment was used to acquire CHD $\Delta T$ data at $7.5 \mathrm{~min}$ powered and unpowered transients (ON/OFF) with the DT sampling and storage resolution of $30 \mathrm{~s}$. Simultaneously the actual gravitational flux density was obtained by dividing the gravimetric flow by the sapwood area of the stems. The sapwood area was measured following the same procedures as for Test 2, and the stem segments were verified for uniformity of the sapwood structure to ensure low circumferential variability.

Additionally, two extra repetitions were performed, to verify the reproducibility of the CHD method in NTG correction following the same procedure as in Test 3. 

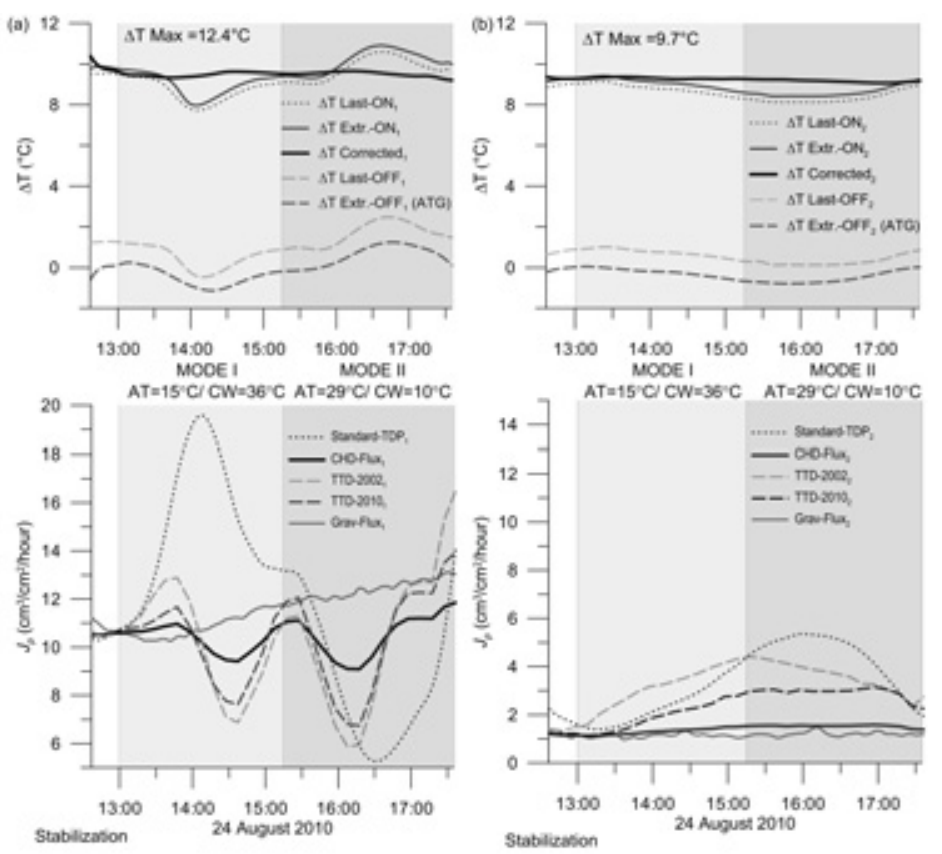

Figure 2.7: Test 3: variability of measured raw $\Delta T$ (above) signals and $J_{p}$ (below) for: (i) Standard-TDP (constantly power TDP measurements); (ii) CHD-Flux (ATG corrected), (iii) TTD 2002 (ATG corrected), (iv) TTD 2010 (ATG corrected); vs. (v) Grav-Flux (gravimetric measurements of $J_{p}$ ) as reference. Test 3 was executed under different, gravimetrically enforced sap flux densities: (a) Segment 1 with $\approx 11 \mathrm{~cm}^{3} \mathrm{~cm}^{-2}$ hour ${ }^{-1}$ and (b) Segment 2 with $J_{p} \approx 1 \mathrm{~cm}^{3} \mathrm{~cm}^{-2}$ hour $^{-1}$. The ATG produced during both ATG enforcement modes is plotted as well (Extr.-OFF). AT, air temperature; $\mathrm{CW}$, circulating water temperature.

\subsubsection{Comparing CHD and TTD methods}

The CHD sap flux densities $\left(J_{p}\right)$ were compared with $J_{p}$ estimates of the TTD method corresponding to different CPS: (i) CPS 10 min ON and 20 min OFF with the calibration of Do and Rocheteau (2002b) -data acquired by CHD extrapolation from 7.5 min raw data; (ii) CPS 10 min $\mathrm{ON}$ and 20 min OFF with the calibration of Isarangkool Na Ayutthaya et al. (2010) -data acquired by CHD extrapolation from $7.5 \mathrm{~min}$ raw data. Additionally, for assessing the fit of the extrapolated ON-OFF cycles, TTD was applied using the raw data (7.5 min ON and $7.5 \mathrm{~min}$ OFF) for both TTD calibrations. For each calibration, the raw and extrapolated TTD $J_{p}$ estimates were consistently similar (10\% difference). Thus, in order to follow the most updated CPS recommendation for both TTD calibrations (Isarangkool Na Ayutthaya et al., 2010), the TTD estimates extrapolated to 10 and 20 min were also used for comparison with the CHD $J_{p}$ and with the reference gravimetric sap flux densities. 
To calculate the TTD calibration of Do and Rocheteau (2002b) (TTD2002) the following equation was used on the extrapolated $\Delta T$ data obtained from Eq. (2.7):

$$
J_{p}=11.3 K_{a} /\left(1-K_{a}\right)^{0.707}
$$

where $J_{p}$ is the sap flux density and $K_{a}$ is a transient flow index. $K_{a}$ was calculated from

$$
K_{a}=\left(\Delta T_{0 a}-\Delta T_{a}\right) / \Delta T_{a}
$$

where $\Delta T_{0 a}$ is the maximum alternated temperature difference under zero flow and $\Delta T_{a}$ is the transient or alternate signal. The alternated power $\Delta T_{0 a}$ value necessary for applying the TTD method was defined from 10 - 15 min extrapolation of an ON cycle under zero flow conditions, using the alternated data collected from both segments. The $\Delta T_{a}$ was defined as

$$
\Delta T_{a}=\Delta T_{\text {on }}-\Delta T_{\text {off }}
$$

where $\Delta T_{o n}$ is the temperature difference reached at the end of a powered cycle and the $\Delta T_{\text {off }}$ is the temperature difference at the end of an unpowered cycle. The $\Delta T_{\text {off }}$ was calculated by averaging the OFF cycles before and after an ON cycle

To calculate the TTD of Isarangkool Na Ayutthaya et al. (2010) (TTD2010), the same set of equations from TTD-2002 was used (Eqs. (2.9)(2.10)), but the multi-species calibration for calculating $J_{p}$ (Eq. 2.11) was used instead.

$$
J_{p}=12.95 K_{a}
$$

\subsubsection{Error calculations}

Three measurements of sap flux density error were used to report the results of the laboratory validation in terms of the disagreement between the gravimetric reference and the estimated sap flux densities. The relative percentage error (\%Error) provided information on the under-overestimations during the experiments in proportion to the magnitude of the gravimetric reference and in time. Such events were indicated as a negative error for overestimations and positive for underestimations. The \%Error is calculated as follows:

$$
\% \text { Error }=\frac{\text { Gravimetric }- \text { Estimated }}{\text { Gravimetric }} \times 100
$$

The second measurement of error, the "residual", is a straightforward measurement of deviation of the estimated data in the same units of measurements (in this study ${ }^{\circ} \mathrm{C}$ ); however, it does not take into consideration the magnitude of the gravimetric flux density. The "residual" is calculated as follows:

$$
\text { Residual }=\text { Gravimentric }- \text { Estimated }
$$


Finally, the mean absolute percentage error (MAPE) was calculated to determine the overall error. The MAPE indicated the level of accuracy for each correction methodology avoiding miscalculations due to selfcancellation of under-overestimations in the whole time series. The MAPE provided a way to objectively compare the assessed methods and was calculated as follows:

$$
\% \text { MAPE }=\frac{100}{n} \sum_{i=1}^{n}\left|\frac{\text { Gravimentric }_{i}-\text { Estimated }_{i}}{\text { Gravimentric }_{i}}\right|
$$

\subsection{Results and discussion}

\subsubsection{Characteristics of the plant material}

Table 2.1 presents the biometric characteristics of the stem segments used during the experiments. From this information it is clear that the TDPs were completely surrounded by xylem tissue and not in touch with the heartwood ( $\geq 2 \mathrm{~cm}$ for all segments).All the stems had regular shape, the stem diameter was on average $8.5 \mathrm{~cm}$, with low variations (std. dev. $=0.6 \mathrm{~cm}$ ), and all the probe installations covered between 50 and $90 \%$ of the cross-sectional sapwood area.

This, together with the low $J_{p}$ radial variability obtained with cut segments under laboratory conditions at $J_{p}<17 \mathrm{~cm}^{3} \mathrm{~cm}^{-2}$ hour ${ }^{-1}$ (Steppe et al., 2010), reduced the probability of $J_{p}$ miss-estimation due to radial water flow variability as pointed out by Clearwater et al. (1999). They concluded that radial variability within $2 \mathrm{~cm}$ of the TDP is more consequential for steep sap flux gradients, which was not the case in our measurements.

\subsubsection{Test 1: confirming ATG formation}

The infrared images confirmed that the ATG enforcement system in Mode I and Mode II was able to generate substantial ATG on stem segments (Figure 2.3a and 2.3b). This can be observed in the colour gradient that stretched from the plastic tubing towards the stems and indicating the direction of conducted heat. In Mode I the ATG enforcement system increased the stem temperature (warmer colours in close contact with plastic tubing in Figure 2.3a), while in Mode II it decreased the stem temperature (cooler colours in close contact with plastic tubing in Figure 2.1b). In both modes, most of the stem surface was primarily affected by air temperature, which was warmer in Figure 2.3b than in 2.3a. The difference between circulating water temperature on the stem and the air temperature forms the ATG.

The enforced temperature conditions of Mode I created an ATG influence revealed by negative $\Delta T$ in Test 1 under no-flow conditions (Figure 2.5). Such influence corresponds to the warm gradient in the infrared image in Figure 2.3a. A negative $\Delta T$ means that the upper probe located 
closest to the tubing (lower probe in the standard TDP installation) had a higher temperature than the lower probe closer to the stem bottom outlet (the one normally heated probe in the standard TDP installation). Such gradient decreases the magnitude of the recorded, biased by ATG, $\Delta T$ values. The Mode I was started at 16:30 with immediate influence of the heat of the ATG enforcement tubing. After 3 h, i.e., at $\approx 19: 30$, the $\Delta T$ reached a point of quasi-stabilization lasting for another $13.5 \mathrm{~h}$ until 09:00 of the next day when $\Delta T$ reached nearly $.3 .{ }^{\circ} \mathrm{C}$ This covered the whole spectrum of negative NTG ( $\Delta T$ biased by temperature gradient) reported so far in field conditions (Do and Rocheteau, 2002a; Lubczynski et al., 2012; Reyes-Acosta and Lubczynski, 2014).

The ATG enforcement of Mode II resulted in an ATG revealed by positive $\Delta T$ in Figure 2.5 and in the cold blue colours in the infrared image in Figure 2.3b. A positive $\Delta T$ means that the upper probe located closest to the tubing was more influenced by the low temperature than the probe closest to the stem bottom outlet (the one normally heated probe in the standard TDP installation). Such a gradient increases the magnitude of the recorded, biased by ATG, $\Delta T$ values. The Mode II started at 09:00 immediately after termination of Mode I (Figure 2.5). Transition to Mode II took place as an abrupt change in ATG due to the reversing of the ATG enforcement conditions. This resulted in a sharp rise of $\Delta T$ from approximately $-3{ }^{\circ} \mathrm{C}$ to $0{ }^{\circ} \mathrm{C}$ in $1.5 \mathrm{~h}$ and further to 1.5 ${ }^{\circ} \mathrm{C}$ within another $1.5 \mathrm{~h}$, i.e., at 12:00, when the semi-stabilization started. The final stabilization at $\Delta T=+2.6 w^{\circ} \mathrm{C}$ as achieved after another $6 \mathrm{~h}$, i.e., at 18:00 when the Test 1 was concluded. This covered the whole spectrum of positive NTG ( $\Delta T$ biased by temperature gradient) reported in field conditions (Do and Rocheteau, 2002a; Lubczynski et al., 2012; Reyes-Acosta and Lubczynski, 2014).

Test 1 showed that the proposed laboratory ATG enforcement system was able to generate large ATG influences on the TDP signal, $\approx 1.5$ times larger than typical field observations of NTGs that approximately vary between -2.5 and $1.5{ }^{\circ} \mathrm{C}$ (Reyes-Acosta and Lubczynski, 2014). Thus, the ATG enforcement system can be considered as a suitable laboratory method to create thermal gradients that bias the $\Delta T$ signals of TDP, and hence it is adequate for validating the CHD method.

\subsubsection{Test 2: understanding the biasing dynamics of ATGs}

Test 2 presented in Figure 2.6 followed three sequential stages: (i) stabilization; (ii) Mode I; and (iii) Mode II. During the initial stage of stabilization the TDP signal reached thermal equilibrium after $\approx 45 \mathrm{~min}$, i.e., at 12:15 (Figure 2.6a) in both segments and continued for $1 \mathrm{~h}$ until 13:15. During this $1 \mathrm{~h}$ period, the $J_{p}$ Standard-TDP1 and Standard-TDP2 in Figure 52.6 reached comparable values to the $J_{p}$ gravimetric measurements Grav-Flux1 and Grav-Flux2, showing a mean underestimation of 3\% for Segment $1\left(16 \mathrm{~cm}^{3} \mathrm{~cm}^{-2}\right.$ hour $\left.^{-1}\right)$ with high flow and $27 \%$ for Segment 2 (2 $\mathrm{cm}^{3} \mathrm{~cm}^{-2}$ hour ${ }^{-1}$ ) with low flow. These results show a better agreement with the gravimetric measurements than the $60 \%$ underestimation found 
by Steppe et al. (2010) for American beech across a range of sap flux densities between 5 and $80 \mathrm{~cm}^{3} \mathrm{~cm}^{-2}$ hour ${ }^{-1}$, using the same validation methodology. This disagreement might be attributed to the type of TDP sensor used by Steppe et al. (2010), a modified version of the original design (30 mm long instead of $20 \mathrm{~mm}$ ) with a probe spacing of $40 \mathrm{~mm}$ (Dynamax Inc., Houston, TX, USA), which does not follow the original design of Granier (1985). In this study, the UP sensors were used for all the tests, which are built following the original Granier design. The good agreement between $J_{p}$ Standard-TDP and $J_{p}$ Grav-Flux without ATG bias proved appropriate TDP performance in optimal conditions.

Mode I was activated at 13:15. In the first $15 \mathrm{~min} \Delta T$ in both segments started to decrease (Figure 2.6a) due to the fast effect of the heat released by the tubing, influencing the upper reference probe, thus continuously decreasing the measured $\Delta T$. A similar fast effect was also observed at the beginning of Test 1 under Mode 1. At about 13:30 $\Delta T$ of Segment 1 (Standard-TDP1 with high $J_{p} \approx 17 \mathrm{~cm}^{3} \mathrm{~cm}^{-2}$ hour ${ }^{-1}$ ) sharply changed its pattern starting to increase. This was likely attributed to the moment when the heat front arrived to the lower heated probe, a process dominated by conduction enhanced by a fast water flow. After $30 \mathrm{~min}$, i.e., at $\approx 14: 00$, the $\Delta T$ pattern changed again sharply, decreasing for another 1 $\mathrm{h}$. This $\Delta T$ reduction was likely attributed to the arrival of the convective heat "dragged" along the stem that gradually homogenized the temperature along the reference and heating probes. This is indicated by $\mathrm{AT}$ gradually reaching a quasi-stabilization at about $11,{ }^{\circ} \mathrm{C}$ observed after 15:00 for $30 \mathrm{~min}$. This stabilization was possible only because: (i) the water flow was fast and constant; and (ii) the temperature conditions for simulating ATG were constant as well (air and stem temperature). Under field conditions the air temperature and $J_{p}$ are highly variable, so NTG is always present but changing.

In contrast, $\Delta T$ of Segment 2 (Standard-TDP2 with low $J_{p} \approx 2 \mathrm{~cm}^{3}$ $\mathrm{cm}^{-2}$ hour $^{-1}$ ) continuously decreased during Mode I due to the effect of the heat released by the tubing, influencing the upper reference probe. A gentle inflection point was observed around 14:00, likely indicating the moment when the moving heat front reached the lower heated probe. From that moment the reduction of the $\Delta T$ was less distinct, although it did not reach the stabilization within the time frame of Mode 1, due to the low flow and therefore low speed of heat redistribution.

At 15:30 the ATG enforcement system was changed to Mode II. A similar pattern was observed as for Mode I but with inverse magnitudes of ÄT for both segments, and likely the same explanation of heat transfer but with cooling instead of heating released from the tubing (Figure 2.6b). During the first $15 \mathrm{~min} \Delta T$ followed an increasing effect for both segments. At 15:45 $\Delta T$ of the Segment 1 (Standard-TDP1 in Figure 2.6) started to decrease until it reached a minimum at 17:25 when it suddenly started to increase towards thermal equilibrium. From 16:30 to 17:30 a series of peaks can be observed, but this was likely due to external disturbances caused by entering and exiting the growth room where the experiments took place. In the case of Segment 2 (Standard-TDP2), after 
a time lag of about $15 \mathrm{~min}, \Delta T$ started to increase continuously. As of 17:00, this increasing effect slightly diminished.

The maximum overestimations of $J_{p}$ as compared with the gravimetric controls (Grav-Flux1 and Grav-Flux2) during Test 2 were (Figure 2.6b) 1.76 times for Standard-TDP1 and $>8$ times for Standard-TDP2. The minimum underestimations of $J_{p}$ were 0.4 times for Standard-TDP1 and 0.6 times for Standard-TDP2 (Table 2.2). Do and Rocheteau (2002a) calculated the overestimating effect of NTG upon TDP as $>100 \%$. Lubczynski et al. (2012) found that for low flows $\left(1-2 \mathrm{~cm}^{3} \mathrm{~cm}^{-2} \mathrm{hour}^{-1}\right)$ the NTG bias created overestimations of 6 to 8 times higher than the control.

Table 2.2: Basic statistics of sap flux density measurements and estimates for Test 2 excluding stabilization period.

\begin{tabular}{llllll}
\hline Statistics & \multicolumn{2}{l}{$\begin{array}{l}\text { Grav-Flux in } \\
\mathrm{cm}^{3} \mathrm{~cm}^{-2} \text { hour }^{-1}\end{array}$} & & \multicolumn{2}{l}{$\begin{array}{l}\text { Standard-TDP flux in } \\
\mathrm{cm}^{3} \mathrm{~cm}^{-2} \text { hour }^{-1}\end{array}$} \\
\cline { 2 - 3 } \cline { 6 - 7 } \cline { 5 - 6 } & Segment & Segment & & Segment & Segment \\
\cline { 2 - 3 } \cline { 5 - 6 } & 1 & 2 & & & \\
\hline Max & 18.30 & 2.35 & & 32.16 & 22.33 \\
Min & 15.23 & 1.60 & & 9.04 & 0.60 \\
Mean & 16.32 & 1.84 & & 15.82 & 8.76 \\
Std. & 0.79 & 0.18 & & 4.61 & 7.75 \\
\hline
\end{tabular}

The results of Test 2 showed that: (i) the ATG enforcement system was able to create ATG biases of $J_{p}$ even higher than those affecting TDP sensors in field conditions; (ii) the TDP sap flux density estimates had good agreement with the gravimetric measurements for both segments in the absence of ATG influence; (iii) the ATG bias upon the TDP signal depends on how strong the temperature gradient is and on the magnitude of $J_{p}$ (stronger bias at low $J_{p}$ than at high $J_{p}$ ); (iv) with higher $J_{p}$ the time to reach thermal stabilization is shorter; and (v) the effect of the created ATG upon the measured $\Delta T$ was detected quite early in the experiment, due to the combined effect of heat convection and conduction.

\subsubsection{Test 3: assessing the accuracy of CHD to correct the ATG bias}

The CPS data needed to calculate the CHD correction were processed with an automatic routine in which each ON/OFF interval was extrapolated to steady-state equilibrium according to a physical model (Lubczynski et al., 2012) optimized with a least-squared method algorithm. The optimization provided a set of calibrated $A$ and $T$ parameters, for Eqs. (2.5)-(2.7). The statistical residuals of the extrapolations (measurement of deviation between the simulated and measured signals) were carefully verified to avoid sub-optimal or ill-posed calibrations. The processed CHD data consist of three outputs: (i) the ATG obtained by extrapolation of the OFF intervals according to Eqs. (2.5)-(2.7) averaged with Eq. (2.4); (ii) ATG biased $\Delta T$ obtained by extrapolation of intervals $\mathrm{ON}$ as per 
Eq. (2.5), corresponding to the signal of constantly powered scheme (i.e., Standard-TDP); and (iii) ATG-corrected $\Delta T$ according to Eq. (2.3). Outputs (ii) and (iii) were further processed using Granier's calibration to obtain the $J_{p}$ estimates of ATG biased (Standard-TDP) and ATG corrected (CHD-Flux), respectively (Figure 2.7).

The ATG-biased $\Delta T$ signal of both stem segments $\left(\Delta T\right.$ Extr.-ON ${ }_{1}$ in Figure 2.7a and $\Delta T$ Extr.-ON $\mathrm{ON}_{2}$ in Figure 2.7b) followed similar patterns as in Test 2 for Mode I and II: (i) Segment 1 (faster water flow) in Test 3 showed a double peak that started in Mode I and reversed in Mode II, and (ii) for Segment 2 (slower water flow) a parabolic shape. These results indicated that the combined effect of (i) the heat conduction due to the ATG enforcement, and (ii) the heat convection due to the flowing water took place during Test 3 as well.

After the stabilization period $\Delta T$ Extr.-ON $\mathrm{N}_{1}$ during Mode I showed an initial decrement for $1 \mathrm{~h}$, between 13:00 and 14:00, similar to $\Delta T$ Standard-TDP 1 in Test 2 but less steep, between 13:00 and 13:30 in Figure 2.6b. It corresponds to the increase of the temperature of the upper reference probe, thus a decrease of $\Delta T$. At 14:00, the $\Delta T$ Extr.-ON ${ }_{1}$ reversed and started to increase as a result of the arrival of the heat dragged from the tubing to the lower probe. After about $1 \mathrm{~h}$ a quasi-stabilization at 9 ${ }^{\circ} \mathrm{C}$ was achieved due to the gradually homogenized temperature along the distance between the reference and heating probes. The stabilization period lasted for another $0.5 \mathrm{~h}$ after which Mode I was terminated. In Mode II of Test 3, the Segment 1 had similar behaviour but opposite pattern, which was explained by similar heat transfer mechanisms but with a cooling effect of the tubing instead of warming and opposite air temperature.

For Segment 2 in Mode I and II, $\Delta T$ Extr.- $\mathrm{ON}_{2}$ followed a similar pattern as $\Delta T$ Standard-TDP 2 in Test 2 but the range of magnitudes of $\Delta T$ was different (Test 2 ranged between 7 and $11{ }^{\circ} \mathrm{C}$ and Test 3 between 8.5 and $9.5^{\circ} \mathrm{C}$ ). This difference could be attributed to differences in $J_{p}$ $\left(\approx 1 \mathrm{~cm}^{3} \mathrm{~cm}^{-2}\right.$ hour ${ }^{-1}$ in Test 1 and $\approx 2 \mathrm{~cm}^{3} \mathrm{~cm}^{-2}$ hour ${ }^{-1}$ in Test 2$)$ and the differences in the length and the sapwood tissue arrangement of both segments. Segment 2 used in Test 3 had a considerably larger sapwood depth (50\% larger) than the segment of Test 2 (see Table 2.1).

The $\Delta T$ OFF measurements extrapolated to steady-state thermal equilibrium reflecting the ATG bias in Mode I and Mode II ( $\Delta T$ Extr.-OFF 1 and $\Delta T$ Extr.-OFF 2 ) in general followed the pattern observed for the $\Delta T$ OFF ATG-biased in Test 1, but the magnitudes were different (Test 1 was between -3 and $2.3{ }^{\circ} \mathrm{C}$ whereas Test 3 was between -1.5 and $\left.1.5{ }^{\circ} \mathrm{C}\right)$. This difference could be explained by three possible reasons: (i) for Test 1 the gravimetric $J_{p}$ was kept at zero thus the heat convection was minimal, limiting heat exchange and therefore enlarging ATG; (ii) the length of the stem segment in Test 1 was $10 \mathrm{~cm}$ shorter than the two segments of Tests 2 and 3 (see Table 2.1), implying that the tubing was $\approx 3 \mathrm{~cm}$ closer to the reference probe; and (iii) as a previous example indicated different thermal properties of the stem segments is another possible reason.

By comparing $\Delta T$ Extr.-ON (with ATG bias) and $\Delta T$ Corrected (without 
ATG bias) versus $\Delta T$ Extr.-OFF (representing the ATG bias), it can be seen that the CHD correction removed the parabolic ATG bias patterns resulting in an unbiased nearly straight line of the corrected signal (Figure 2.7). This $\Delta T$ Corrected signal resulted in $J_{p}$ estimates (CHDFlux) that were in good agreement with the gravimetric measurements (Grav-Flux ${ }_{1}$ and Grav-Flux 2 ) as seen in Figure 2.7, the boxplot in Figure 2.8 and the accumulated water calculations in Figure 2.9. The CHD-Flux 1 and CHD-Flux ${ }_{2}$ covered the maximum and minimum values of the actual sap flux densities satisfactorily (Grav-Flux 1 and Grav-Flux 2 ) as can be observed in Table 2.3 and Figure 2.8. The results for Test 3 showed that the CHD method works better when the heat convection is high, as is the case at high sap flux densities. The repetitions of Test 3 showed the same patterns described for Mode I and Mode II during Test 3.
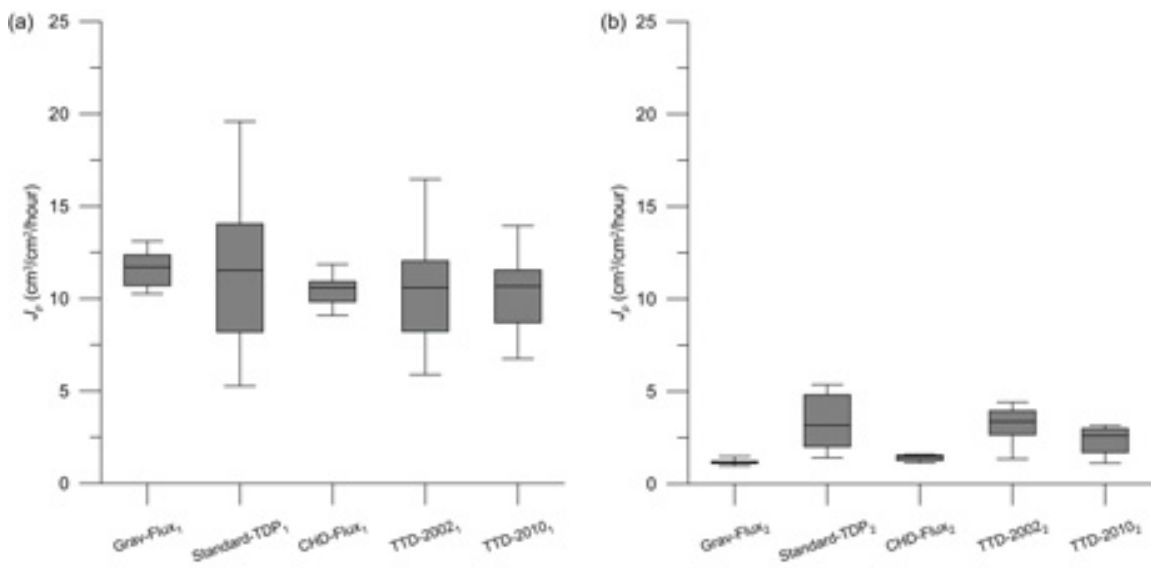

Figure 2.8: Boxplot for the corrections obtained during Test 3 vs. the gravimetric reference (Grav-Flux) and uncorrected data (Standard-TDP), for (a) Segment 1 and (b) Segment 2.

Table 2.3: Basic statistics of the sap flux density measurements and estimates for Test 3, using different correction methods: CHD-Flux 7.5 min; TTD $7.5 \mathrm{~min}$; and TTD 10-20 min. Segment $1=11 \mathrm{~cm}^{3} \mathrm{~cm}^{-2}$ hour $^{-1}$ and Segment $2=1 \mathrm{~cm}^{3} \mathrm{~cm}^{-2}$ hour $^{-1}$.

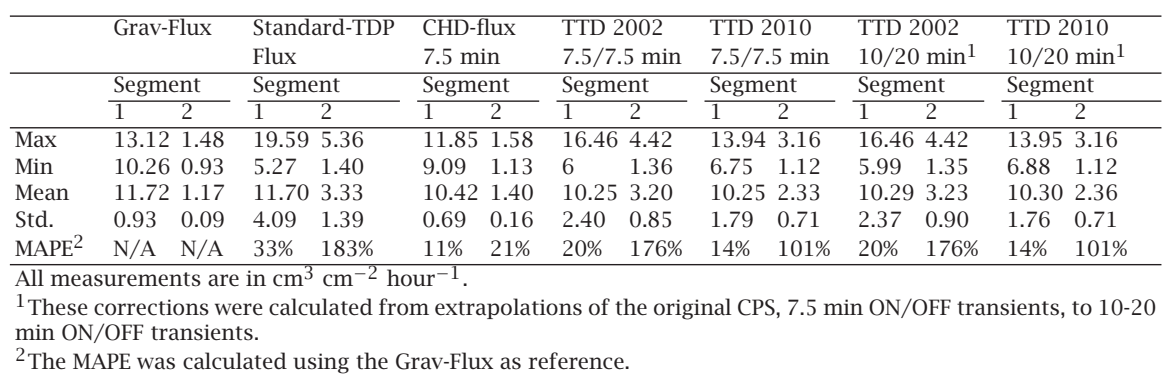



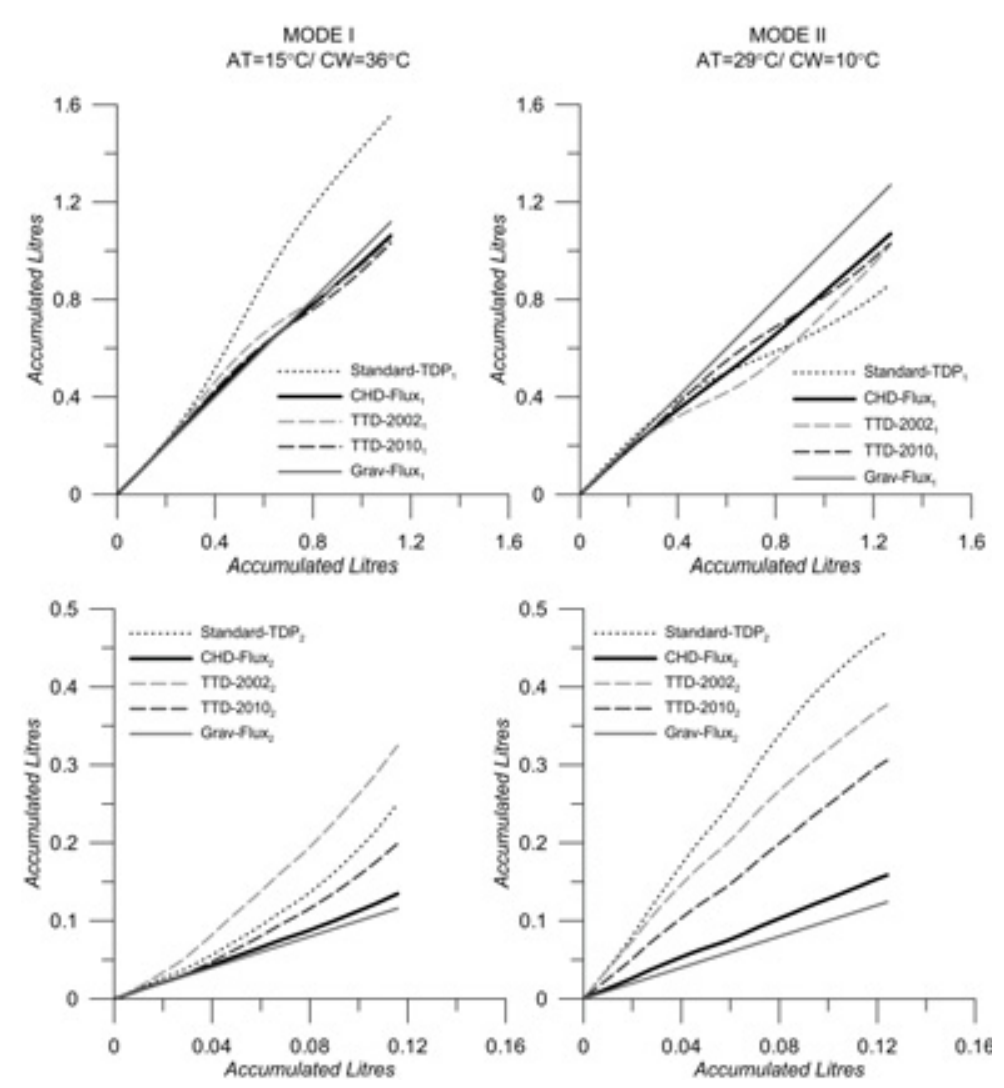

Figure 2.9: Accumulated water estimates, calculated for Standard-TDP (constant power) and CHD, TTD-2002 and TTD-2010 for both segments (upper figures $=$ Segment 1 and bottom figures $=$ Segment 2 ) in ATG Mode I and II, vs. gravimetric reference measurements (Grav-Flux). AT = air temperature and $\mathrm{CW}=$ circulating water temperature.

An important consideration for the results obtained in Test 3 is that a single set of TDP sensors is not sufficient for field measurements in trees with significant circumferential variability.In such trees it is necessary to use at least three sets of TDP sensors but optimally four TDP sets in four cardinal directions, i.e., north, west, south and east. Moreover, each individual measurement must be corrected for NTG, which varies circumferentially as well. Unfortunately, this multi-sensor setup was difficult to implement because there was not enough room to install more TDP sensors. The surface of the stem segments was covered by the following components: (i) the space required for the installation of Mariotte system, (ii) the space for the cable ties holding the stem segment to the Mariotte structure, (iii) the space required for plastic tubes circulating the water, and (iv) a wide-enough "free" area in which we could monitor the formation of the ATG with the infrared camera. Because of these constrains only one set of TDP sensors was 
used. However, to minimize the eventual $J_{p}$ circumferential variability two procedures were used. First, a test was performed without ATG to verify the agreement between the $J_{p}$ measured by the TDP and the $J_{p}$ of the entire segment obtained by the gravimetric method. Second, after the test, the uniformity of the xylem was verified by microscopic inspections of stained segments following Čermák et al. (2000) and Saveyn et al. (2008) who related homogeneous distribution of sapwood with lower circumferential variability. The staining of the sapwood was carried out after the test to avoid the risk of clogging the conducting vessels. The stem segments used in Test 3 yielded a gravimetric $J_{p}$ in good agreement with the $J_{p}$ of TDP and had uniform sapwood structure, thus suggesting low circumferential variability and confidence in the obtained results.

Test 3 concluded that the CHD method satisfactorily corrected the TDP signal biased by ATG, producing a sap flux density estimate in good agreement with the gravimetric measurements. This confirms the adequacy and usefulness of CHD to accurately correct NTG biases of the standard TDP signals acquired in field conditions.

\subsubsection{Comparing cyclic power correction methods}

By observing the results of the cyclic power correction methods in Figures 2.7 and 2.8, it was clear that TTD-2002 (Do and Rocheteau, 2002a,b) and TTD-2010 (Isarangkool Na Ayutthaya et al., 2010) methods reduced the biasing effect of the ATGs upon the sap flux density estimations as compared with non-corrected data (Standard-TDP). During Test 3 the TTD-2010 for both CPS (7.5/7.5 min and 10/20 min) had MAPE of $14 \%$ for Segment $1\left(11 \mathrm{~cm}^{3} \mathrm{~cm}^{-2}\right.$ hour $\left.^{-1}\right)$ and 101\% for Segment $2\left(1 \mathrm{~cm}^{3}\right.$ $\mathrm{cm}^{-2}$ hour $^{-1}$ ). However, the TTD-2002 for both CPS had an error of 20\% for Segment 1 and 176\% for Segment 2 (Table 2.3). These results showed that: (i) both methods handled the ATG bias; (ii) both methods had the highest errors at low flows; and (iii) the TTD-2010 had better accuracy than TTD-2002. This was in agreement with the findings of Isarangkool Na Ayutthaya et al. (2010), and the better performance of TTD-2010 is likely due to better calibration.

The comparison of the CHD with the TTD methods shows that the CHD had the most accurate (Figures 2.8 and 2.9 and lowest MAPE in Table 2.3) and smoothest (Figure 2.7) sap flux density estimates as compared with the gravimetric reference, likely because: (i) the CPS routine is extrapolated to thermal equilibrium while the TTD method uses thermally non-equilibrated cycle endpoints prone to error (Nourtier et al., 2011); and (ii) TTD calibrations (Do and Rocheteau, 2002b; Isarangkool Na Ayutthaya et al., 2010) have not been tested on the species used in this study (F. sylvatica). In terms of accumulated water flow, the TTD methods showed comparable results as the CHD for Segment $1\left(11 \mathrm{~cm}^{3}\right.$ $\mathrm{cm}^{-2}$ hour $^{-1}$ ), but the TTD largely overestimated accumulated water in Segment 2 at low flows $\left(1 \mathrm{~cm}^{3} \mathrm{~cm}^{-2}\right.$ hour $\left.{ }^{-1}\right)$ in both ATG enforcement modes (Figure 2.9). 
The CHD method has the following main advantages over the TTD method: (i) the extrapolation to steady state permitted coping better with the ATG influence and fast temporal variations (Figure 2.7); (ii) the CHD uses the original Granier calibration that applies stable steady state as principle and is widely verified on various species; and (iii) more reliable $J_{p}$ estimates, particularly for low flows. In contrast, the disadvantage of the CHD is the complex and large amount of data to be acquired.

Currently, the choice of using TTD or CHD depends on the objectives and environments where the sap flow measurements are taking place. If the objective is to measure accurately species with highly variable sap flow and located in areas where high NTGs are likely (e.g., arid, semi-arid, very-dry and open forests), CHD is the optimal choice. Conversely, if the measurements are taking place in areas with lower NTGs with species of low sap flow variability and the accuracy of the measurements is not a priority, the TTD method might be easier to apply (e.g., closed forest with considerable water availability). Nevertheless, future improved applications applying powerful automatic parameter optimization techniques used in hydrological modelling (Vrugt et al., 2003a; Vrugt and Robinson, 2007), such as the SCEM-UA (Vrugt et al. 2003b) or DREAM (Laloy et al., 2010), would decrease the processing time substantially making the CHD method more user friendly and simple enough to be used effectively in any areas, including those with lower NTGs as well.

\subsubsection{Limitations of the CHD method for correcting ATGs}

The results showed that the CHD method performed better at correcting ATG biases than other cyclic-power methods. However, the tests also highlighted two situations in which the effectiveness of CHD decreased and the percentage of error was higher than an acceptable range of $15 \%$ as compared with gravimetric measurements (Figure 2.10): (i) when ATG $\Delta T>1.0{ }^{\circ} \mathrm{C}$ and $J_{p}=11 \mathrm{~cm}^{3} \mathrm{~cm}^{-2}$ hour $^{-1}$ (Segment 1) during the moment of change from maximum ATG in Mode I towards maximum ATG of Mode II (16:30 to 18:00 in the error time series of Figure 2.10a); and (ii) when ATG $\Delta T<-0.5{ }^{\circ} \mathrm{C}$ at a low $J_{p}=1-2 \mathrm{~cm}^{3} \mathrm{~cm}^{-2}$ hour ${ }^{-1}$ (Figure 2.10b).

The error measurements during Mode I and Mode 2 followed in general the patterns described for Test 2 and Test 3, increasing and decreasing according to the ATG bias, but were especially sensitive during $\Delta T$ changes. For example, at about 14:15 (Figure 2.10a), when ATG $<.1 .0\left({ }^{\circ} \mathrm{C}\right.$ Mode I) and $J_{p}=11 \mathrm{~cm}^{3} \mathrm{~cm}^{-2}$ hour $^{-1}$ (Segment 1$)$, the CHD correction reduced the bias but still the error was $>10 \%$. This period corresponds to the moment of thermal change, and continued until quasistabilization at 15:15 (Figure 2.10a) when the error decreased to $<10 \%$ during $15 \mathrm{~min}$. At 15:30, when ATG $>1.0\left({ }^{\circ} \mathrm{C}\right.$ Mode II) and $J_{p}=11 \mathrm{~cm}^{3} \mathrm{~cm}^{-2}$ hour $^{-1}$ (Segment 1), the error increased again $>10 \%$ and reached a maximum of $25 \%$ at 16:30. After this point the error started to decrease and at 17:00 it stabilized at $\approx 10 \%$ for the rest of the test. To explain the precise reason for these two peaks of error is 
(a)

a) $+++4 \operatorname{sentand}-10 \mathrm{P}$

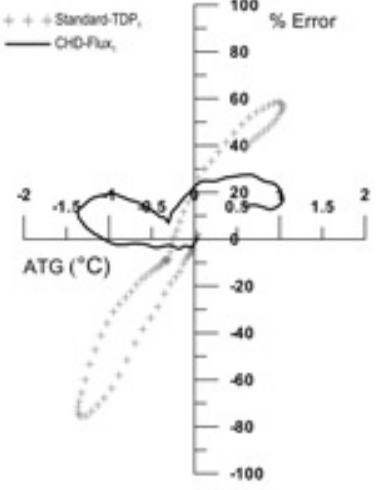

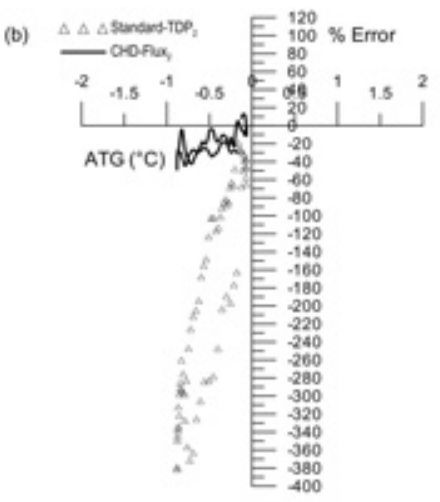
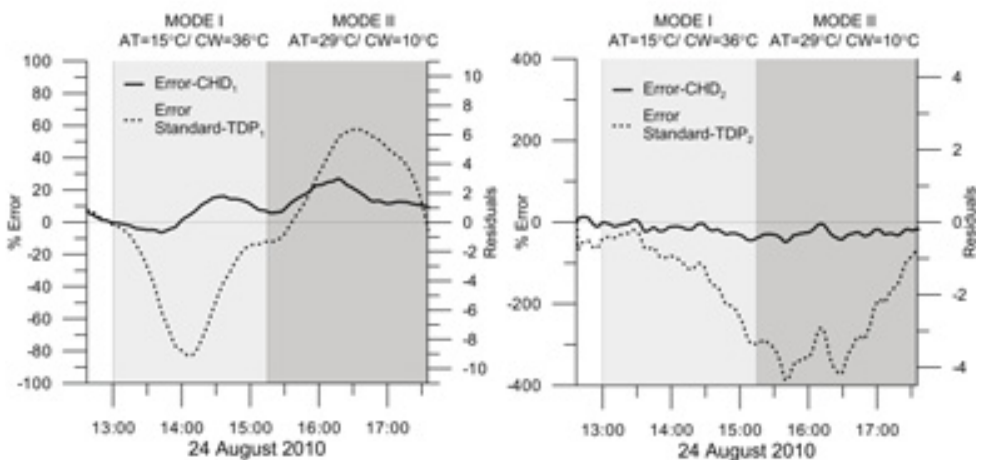

Figure 2.10: Relative percentage error calculated from gravimetric measurements versus ATG (above) and relative percentage error/residuals in time (below) influencing $J_{p}$ from CHD-Flux, in Segment 1 (a) and Segment 2 (b). The error obtained from the Standard-TDP is displayed as an example of a biased signal to highlight the performance of the CHD. AT = air temperature and $\mathrm{CW}=$ circulating water temperature.

difficult, but a likely explanation is that the CHD method could not keep up with the speed of the temperature change in the stem caused by the ATG enforcing system in both modes, because: (i) the time resolution of the cycle power scheme (7.5 min ON-OFF) was not high enough to account for this fast change, and/or (ii) the optimization of the $A$ and $T$ parameters of the CHD produced poorer fits in those specific time periods of fast temperature change. These patterns were observed in the repetitions as well, but they do not constitute a drawback on the method for field implementation. In natural conditions the differences of temperature are more gradual than with the ATG enforcing system, and thus the CPS resolution is more likely to cover the temporal variability of the temperature gradients and produce better fits for the $A$ and $T$ parameters.

For Segment 2, with a sap flux density of $1 \mathrm{~cm}^{3} \mathrm{~cm}^{-2}$ hour ${ }^{-1}$, the CHD correction did clearly decrease the overestimation error, but when 
the ATG was $<-0.5\left({ }^{\circ} \mathrm{C}\right.$ ATG enforcement set in Mode II), the error increased and reached a maximum of $-40 \%$ at 15:30 (Figure 2.10b). After this point the error started to decrease and stabilized towards $-20 \%$ for the rest of the test. This implies that that despite much lower residual error in Segment 2 as compared with Segment 1, its relative percentage error was much larger (Figure 2.10). The described situations when the error was $>15 \%$ and $<-15 \%$ are particularly relevant if the objective of the study requires accurate high temporal resolution sap flux density measurements (e.g., minutes to hours), but for a longer time water flow integration (e.g., daily variation), small under- and overestimation errors can cancel out as mentioned by Braun and Schmid (1999) and Köstner et al. (1998).

The MAPE for Test 3 showed that CHD-Flux 1 had an 11\% error, whereas the constantly powered Standard-TDP 1 had $33 \%$ error. In the case of Segment 2, the CHD-Flux 2 had $21 \%$ error and the constantly powered Standard-Flux 2 183\% error. The higher error for CHD-Flux in Segment 2 as compared with Segment 1 does not indicate that the CHD failed in correcting the ATG influence, but that at high $J_{p}$ values the correction is more accurate. Thus, the difference between the MAPE calculations of both segments might be explained by the difference in sap flux densities. This is the case, because the thermal gradients are likely to stabilize faster at higher sap flux densities than at low densities. Hence, the error at low sap flux densities after CHD correction could be caused by: (i) at lower flow there is lower convection so the the ON/OFF extrapolations require longer time for stabilization and thus fitting of the curves is more difficult and prone to error; (ii) the stem segment for these tests having a deeper sapwood depth $(3.6 \mathrm{~cm})$ than the length of the TDP probe; thus, that section of the sapwood area, albeit smaller than the initial section, was unmeasured; and (iii) inherited error due to the poorer accuracy of TDP probes at low flows as discussed by Braun and Schmid (1999).

At the end of Test 3, the CHD-Flux ${ }_{1}$ showed a poorer performance at correcting the NTG bias in Mode II as compared with Mode I (Figure 2.10a), so as a result the $J_{p}$ was underestimated (Figure $2.7 \mathrm{a}$ ). However, the lower performance in such situations would not importantly affect the overall sap flux density estimation because at night the flow is normally zero or very low (Lu et al., 2004; Regalado and Ritter, 2007). This means that the CHD method corrects the ATG bias the best before sunrise (when the $\Delta T_{\max }$ is normally defined) and during the peak of water flow, when the heat convected by water flow is more important.

In spite of the appointed limitations, the CHD methodology was confirmed to be the most appropriate method to reduce the bias caused by the ATGs. 


\subsection{Conclusions}

Accounting for NTG constitutes one of the most important biasing aspects to consider when applying TDP in semi-arid environments with open vegetation. Thus, the results gathered with this study are an important contribution towards the understanding of NTG biases and appropriate ways to account for it in semi-arid vegetation. It was concluded that (i) the ATG enforcement system is appropriate to generate ATG biases on the TDP signal under controlled laboratory conditions at various sap flux densities (similar to NTGs observed under field conditions); (ii) TDP is highly sensitive to the ATG bias; (iii) the assessed bias is dependent on the sap flux density and the strength of the applied ATG; and (iv) the CHD method constitutes a satisfactory method to remove the influence caused by varying and contrasting temperature gradients at different sap flux densities and resulted in generally more accurate estimates than other cyclic power methods. Finally, this study verified the relation of the magnitude of the sap flux densities to the NTG bias. Such information provided a better understanding on the dynamics governing the NTG influence and susceptibility upon the TDP signal. 



\section{Sap flow measurements optimisation under dry-conditions}

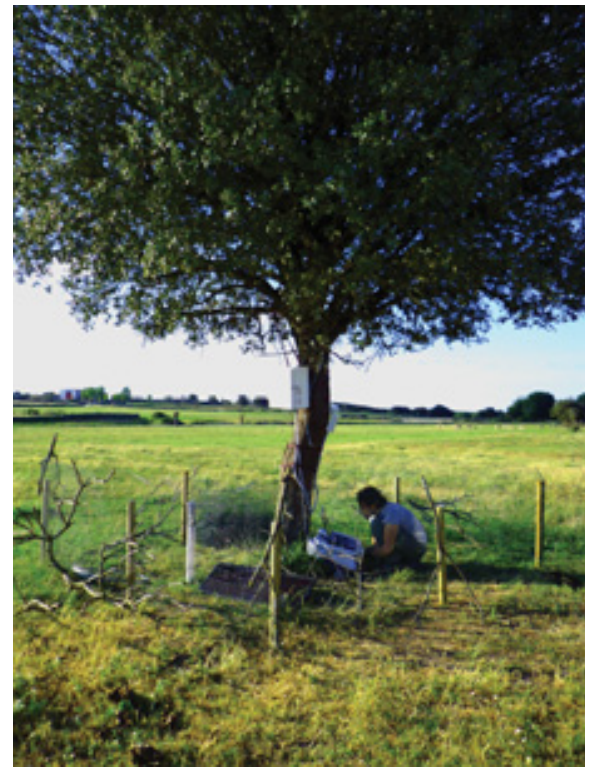

Figure 3.1: Sap flow measurements in a Quercus ilex tree during early dry-season (June) at the Sardón catchment in Salamanca, Spain.

This Chapter is based on: Optimization of dry-season sap flow measurements in an oak semi-arid open woodland in Spain. (2014). Published in Ecohydrology. 


\begin{abstract}
In sap flow studies there is no method complying with high efficiency and versatility of measurements. Thus, to improve the sap flow measurements, we propose combining two methods: (i) thermal dissipation probes (TDP) known to be efficient and cost effective and (ii) heat field deformation (HFD) known to be versatile. For that purpose we used a step-wise TDP sap flux density $\left(J_{p}\right)$ optimisation method consisting of: a) natural temperature gradient (NTG) correction applying the cyclic heat dissipation method; b) $\Delta T_{\max }$ night flow correction using HFD data as reference and c) radial-azimuthal correction using HFD as reference. We applied this optimisation on Quercus ilex (Q.i.) and Quercus pyrenaica (Q.p.) trees in a Spanish semi-arid open-woodland during dry-season. The NTG correction resulted in substantial reduction of $J_{p}$ as compared to standard TDP The subsequent $\Delta T_{\max }$ night flow correction resulted in increase of NTG-corrected $J_{p}$ and good agreement with the outermost $2 \mathrm{~cm}$ of HFD for both species. The final radial-azimuthal adjustment of TDP $J_{p}$ resulted in good agreement with HFD $J_{p}$ but also provided novel Q.i. and Q.p. ecohydrological insights such as: (1) continuous sap night-flow in all Q.p. trees and few Q.i. trees; (2) exponentially reduction of $J_{p}$ with sapwood depth and significantly larger $J_{p}$ in the outermost $1 \mathrm{~cm}$ of sapwood for Q.p. than for Q.i.; and 3) high azimuthal $J_{p}$ variability for Q.i. and Q.p. This study confirms practicality and appropriateness of the step-wise optimisation method and contributes to better understanding of water-related dynamics of Q.i. and Q.p. under water-stress conditions.
\end{abstract}

\title{
3.1 Introduction
}

Thermal methods are the most common and accepted techniques to measure sap flow, i.e. flowing water in conductive tissues of plants The sap flow methods are typically applied to understand the wateruse of a diversity of plants, but specifically to estimate transpiration fluxes from trees (Granier, 1987; Köstner et al., 1998; Oltchev et al., 2002b; Lubczynski, 2009) in various fields such as: plant physiology (Nadezhdina, 1999; Čermák et al., 2000, 2008; Nadezhdina et al., 2009), forest management (Čermák et al., 1998; Köstner et al., 1998; Wilson et al., 2001; Hernández-Santana et al., 2008a), hydrology (Lubczynski, 2000; David et al., 2004; Lubczynski and Gurwin, 2005), ecology (Wullschleger et al., 2001; Burgess and Dawson, 2004), etc. However, there is a general concern about the accuracy and adequacy of these methods when used to quantify the actual amount of water used by plants or in higher levels of organization such as tree stands or catchments. The thermal methods are prone to inaccuracies if certain aspects of tree dynamics are not accounted for, such as: natural temperature gradients (Do and Rocheteau, 2002a; Lu et al., 2004), night flow (Grime and Sinclair, 1999; Regalado and Ritter, 2007), azimuthal variability of the sap flow, radial variability of the sap flow (Čermák et al., 2004; Lu et al., 2004), reverse-flow (Burgess et al., 2000). Each inaccuracy can be accounted for with the appropriate method 
but there is not a single universal thermal method that can handle all the inaccuracies. Therefore various measuring schemes combining different methods and/or involving mathematical modelling have been developed (Čermák et al., 2004; Steppe et al., 2006; Čermák et al., 2007; Steppe and Lemeur, 2007b; De Pauw et al., 2008). This study is inline with such developments. We propose combining cost effective and robust thermal dissipation probe (TDP) (Granier, 1985) sap flux density $\left(J_{p}\right)$ measurements with the versatile heat field deformation (HFD) $J_{p}$ measurements.

The TDP method has five potential inaccuracies that should be taken into account while quantifying $J_{p}$ : (1) in standard implementation the TDP method is vulnerable to natural temperature gradient (NTG) bias resulting in data that reflects changes not only related to the target heatconvection of flowing water, but also to the measurement of NTG-related heat-conduction bias (Čermák and Kučera, 1981; Do and Rocheteau, 2002a,b; Lu et al., 2004); (2) when using TDP it is necessary to define zero flow condition, corresponding with the maximum measured temperature difference $\left(\Delta T_{\max }\right)$ usually observed at night or pre-dawn; however in trees presenting night flow assigning $\Delta T_{\max }$ without correction leads to an incorrect estimation of sap flow (Lu et al., 2004; Regalado and Ritter, 2007); (3) TDP measurements cannot provide radial and azimuthal variability of $J_{p}$ (Lu et al., 2004) in trees with sapwood depth $>2 \mathrm{~cm}$; (4) standard TDP does not measure bi-directional flows, e.g. hydraulic redistribution (Burgess et al., 2000); (5) the TDP method uses an empirical calibration (Granier, 1985) relating measured $\Delta T$ to $J_{p}$ that is appropriate for diffuse-porous sapwood tree species, but according to Bush et al. (2010) ring-porous tree species might not follow such calibration (Reis et al., 2006; Steppe et al., 2010). The first three listed TDP inaccuracies are handled in this study; the fourth and fifth are not because we did not experience any important bi-directional flow as defined by HFD method and because the European oaks have a good and reliable TDP verification record (Granier et al., 1994; David et al., 2004; Hernández-Santana et al., 2008a,b).

The HFD method has three advantages as compared to TDP, it can better detect night flow, it provides radial variability of $J_{p}$, in current implementation up to $8 \mathrm{~cm}$ of xylem depth and it measures bi-directional flows (Nadezhdina et al., 1998; Cermák et al., 2004). The HFD method has already been used in studies involving species from the Quercus genera (David et al., 2007; Poyatos et al., 2007; Nadezhdina et al., 2008), showing good results. The HFD measurement is species-independent (Nadezhdina et al., 2002) and due to its technical principle, it is not affected by sudden changes of air temperature (Nadezhdina et al., 2004; Tributsch et al., 2006). However, the HFD sensors are difficult to construct, so costly, which limits their use at studies that require parallel sap flow measurements at several trees.

The two main objectives of this study are as follows: (1) to develop step-wise optimisation method of TDP sap flux density measurements to handle the following drawbacks: (a) NTG bias; (b) $\Delta T_{\max }$ bias due to the 
night flow; (c) radial and azimuthal $J_{p}$ variability; and (2) to enhance the available knowledge on the dry season water-use dynamics of Quercus ilex ssp. ballota (Desf.) Samp (Q.i.) and Quercus pyrenaica Willd (Q.p.) tree species in water limited environments.

\subsection{Materials and methods}

\subsubsection{Study area}

We carried out two sap flow field campaigns in the dry seasons of 2009 and 2010, each from June to September, in the Sardón catchment $(\approx 80$ $\mathrm{km}^{2}$ ) near Salamanca (Spain). The sap flow measurements were taken in the northern part of the Sardón catchment described by Lubczynski and Gurwin (2005) in the surrounding of the Trabadillo meteorological station $\left(6^{\circ} 8^{\prime} 50.302\right.$ ” W $41^{\circ} 7^{\prime} 5.593$ ”N). The climate of the Sardón study area is semi-arid, with a mean rainfall of $\approx 500 \mathrm{~mm} \mathrm{yr}^{-1}$ (Lubczynski and Gurwin, 2005). The warmest and the driest months are July and August with an average temperature of $22,{ }^{\circ} \mathrm{C}$ potential evapotranspiration (PET) of $5 \mathrm{~mm} \mathrm{day}^{-1}$ and rainfall $<3 \mathrm{~mm}$ month $^{-1}$. The Sardón catchment is composed of massive, fractured and weathered granites. The superficial, weathered, unconsolidated material is only a few meters thick and is widely intercalated by solid granite outcrops. In valleys the groundwater table is low and hydraulically connected with intermittent streams, whereas at topographic elevations it is higher but rests only few meters below the ground surface. The study area is covered by woody-shrub vegetation (Cytisus scoparius also known as Scotch broom) and semi-natural open forest with two dominant tree species: evergreen oak Quercus ilex ssp. ballota (Desf.) Samp (Q.i.), and broad-leafed deciduous oak Quercus pyrenaica Willd (Q.p.). Q.i. has small scleromorphic leaves and is well adapted to drought stress conditions (Manes et al., 2006), whereas Q.p. has broad lobulated leaves and grows rather in sub-humid/humid environments, but it also tolerates dry conditions Hernández-Santana et al. (2008b). The study area is used mainly for pasture because the soils are generally unsuitable for agriculture.

\subsubsection{Monitoring climatic and hydrological variables}

We monitored the following climatic variables using Automatic Data Acquisition Systems (ADAS) based on Campbell CR5000 logger: shortwave incoming and net radiation by CNR radiometer (Kipp \& Zonen, Netherlands), rainfall by Wallingford type of tipping bucket (Eijkelkamp, The Netherlands), and relative humidity, air temperature, wind speed and rainfall by weather transmitter WXT 520 (Vaisala, Finland). To compare sap flow of different days in dry season, we filtered out cloudy days from the climatic data and set a standard to select days under comparable climatic conditions. This standard was defined as follows: clear sky day (no rainfall, no cloud), potential evapotranspiration (PET) $>3 \mathrm{~mm}$ 
day $^{-1}$, vapour pressure deficit $(V P D)>3 \mathrm{kPa}$, soil moisture at 25 and $50 \mathrm{~cm}$ deep $<10 \%$ and smooth parabolic shape of incoming shortwave solar radiation with peak $>700 \mathrm{~W} \mathrm{~m}^{-2}$. Water potential (MPS-1 Decagon, USA) and soil moisture (Stevens-Water probes III, USA) were measured in profiles at 15 min intervals under the canopies of a Q.i. and and a Q.p. tree, each consisting of 4 probes installed at $25 \mathrm{~cm}$ intervals (25- 100 $\mathrm{cm})$.

\subsubsection{Tree survey and stratified sampling for sap flow measurements}

The biometric survey was done by dividing the catchment into 4 macroplots of approximately the same size of $20 \mathrm{~km}^{2}$. In each of the macro-plot, 20 to 25 trees were randomly selected, identified and measured, in order to properly represent the spatial variability of the species. The survey focused on measuring biometric characteristics necessary to define the attributes of the tree population, such as: tree height, diameter at the breast height (DBH), first active branch height, canopy projected area $\left(A_{\mathcal{c}}\right)$, and sapwood area $\left(A_{x}\right)$. These measurements were further processed to define the composition of the population and the DBH was selected as the best parameter to define the population structure. The whole catchment survey was stopped after sampling 90 Q.i. and 84 Q.p. trees, i.e. when the DBH cumulative variability curve stabilized, indicating that the trees variability was properly covered (Figure $1 \mathrm{~b}$ ).

Sap flow measurements, particularly those carried out with HFD, demand a significant amount of extra hardware supplies (e.g. solar panels, batteries, cable-extensions, etc.) and are time consuming. Considering these limitations, 10 trees per species were the maximum that we could monitor for $J_{p}$, (maximum sampling effort). In order to adequately represent the population structure within the chosen set, we applied stratified random sampling (Cochran, 1977) based on the population structure determined for both species (size categories). The resulting schema distributed the 10-trees set, across the DBH size categories, to represent the variability of the whole population (Table 3.1). In the field, the trees were carefully selected by: 1) determining their DBH size category 2) making sure they had symmetrical stems; and 3) verifying that they did not present any apparent sign of decay (hollow trunks) and/or illness (e.g. presence of infected black tissue in core samples).

\subsubsection{Step-wise optimisation of TDP measurements}

The three-step TDP optimisation proposed in this study was applied in a form of step-wise corrections. It followed a strict order: (1) NTG bias correction; (2) night flow $\Delta T_{\max }$ correction and (3) radial-azimuthal correction. In step 1 , the standard TDP measurements $\left(\mathrm{TDP}_{\text {std }}\right)$ were first obtained from non-corrected extrapolations using the Cyclic Heat Dissipation method (CHD) and then corrected for NTG (Lubczynski et al., 2012; Reyes-Acosta et al., 2012) $\left(\mathrm{TDP}_{N T G-\text { free }}\right)$. In step 2 the $\mathrm{TDP}_{N T G-\text { free }}$ 

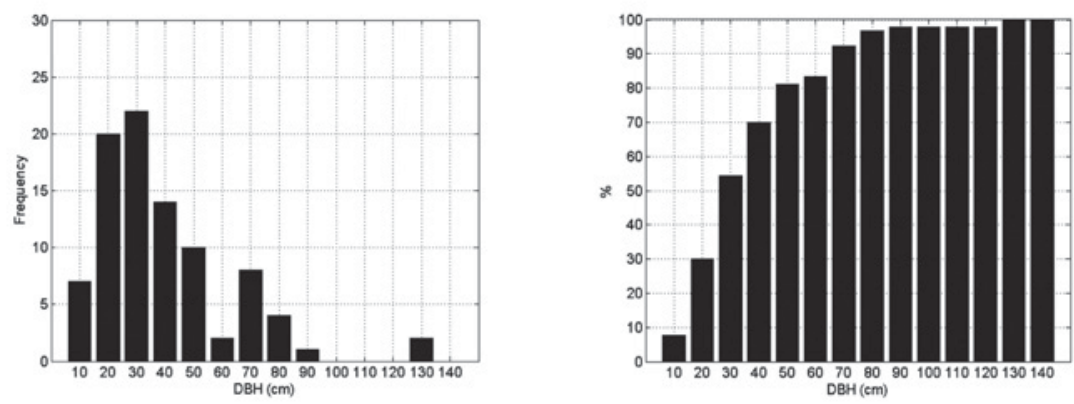

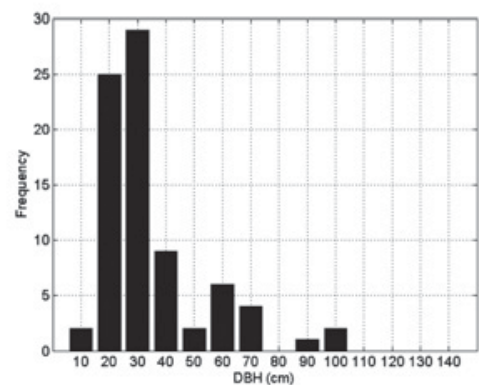

a.)

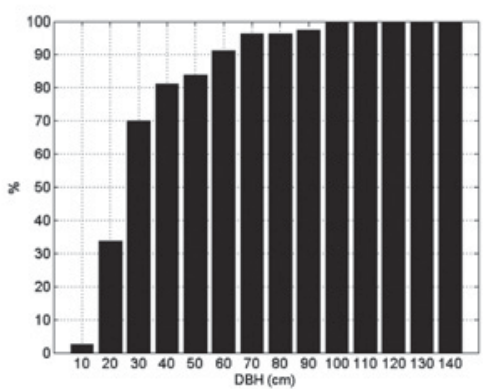

b.)

Figure 3.2: DBH sampling in the Sardón catchment: (a) frequency histogram for DBH size categories; and (b) accumulated frequency curve for DBH size categories obtained from the biometric survey for Q.i. (top) and Q.p. (bottom).

data was corrected for night flow $\Delta T_{\max }\left(\mathrm{TDP}_{\Delta T \max \text {-corr }}\right)$ with help of the HFD reference $\left(\mathrm{HFD}_{\text {ref }}\right)$. Finally, in the step 3 the $\mathrm{TDP}_{\Delta \text { Tmax-corr }}$ data was radially and azimuthally corrected. The obtained TDP final represented the average of the radially and azimuthally corrected $J_{p}$ corresponding with the HFD measurements.

\subsubsection{Step 1: NTG bias correction of TDP measurements by CHD method}

The $\Delta T$ signal of the TDP sensors is sensitive to natural temperature gradient (NTG) which if present, can constitute a large source of error when calculating $J_{p}$ (Čermák and Kučera, 1981; Lundblad et al., 2001; Do and Rocheteau, 2002a; Lu et al., 2004; Tatarinov et al., 2005). Čermák and Kučera (1981) stated that the NTG is caused by several factors, such as: the influence of the temperature variation in the surroundings, the different temperatures of the tree trunk and soil during the sap flow rate increase in the morning, the height of the measuring point above ground, the thickness of the bark, the distance of the tree from the stand margin, the growth irregularities of the tree trunk, and more recently 
Table 3.1: Stratified sampling scheme applied for the dry-season sap flow campaigns of 2009 and 2010.

\begin{tabular}{lll}
\hline $\begin{array}{l}\text { Size category } \\
\text { (DBHcm) }\end{array}$ & $\begin{array}{l}\text { Number of } \\
\text { stratified Q.i. } \\
\text { tree samples }\end{array}$ & $\begin{array}{l}\text { Number of } \\
\text { stratified } \text {.p. } \\
\text { tree samples }\end{array}$ \\
\hline $0-10$ & 0 & 0 \\
$10-20$ & 3 & 3 \\
$20-30$ & 2 & 3 \\
$30-40$ & 1 & 3 \\
$40-50$ & 1 & 0 \\
$50-60$ & 1 & 1 \\
$60-70$ & 1 & 0 \\
$70-140$ & 1 & 0 \\
\hline Total No. & 10 & 10 \\
samples & & \\
\hline
\end{tabular}

Steppe et al. (2005) and Čermák et al. (2007) have proposed heat storage as another factor. Hence, the TDP method is particularly sensitive to NTG if applied in sparsely vegetated woodlands or savannahs and when exposed to highly contrasting day/night temperatures (Lubczynski et al., 2012), a common situation in our Sardón study area in dry seasons.

For the NTG monitoring we used a standard, unpowered TDP systems (UP Gmbh, Germany) on two trees per species $(\mathrm{DBH}=20-50 \mathrm{~cm}$ ) at four cardinal azimuths (North, South, West and East) and three heights (Bottom $=0.50 \mathrm{~m}$, Middle $1.30 \mathrm{~m}$ and Upper $=1.70$ Q.i.- $2.0 \mathrm{~m}$ Q.p.) during eight days (June 2009 and 2010), to define the temporal variability of NTG.

Solutions to minimize or avoid the NTG interference have been proposed. Köstner et al. (1998) suggested to use NTG measurements acquired during previous days to correct TDP measurements acquired in subsequent days. Granier as cited by Köstner et al. (1998) proposed to switch the power supply ON and OFF (cyclic measurements) for 30 minutes, to measure sap-flow (powered measurement) and NTG (upowered measurement) with one hour resolution. Do and Rocheteau (2002a,b) proposed a modification of the original TDP calibration for shorter cyclic measurements of 7 to 10 minutes. Nourtier et al. (2011) proposed to extrapolate 7 to 10 minutes cyclic measurements to thermal equilibrium from non-cyclic measurement of an extra installation in the same tree as reference. Lubczynski et al. (2012) and Reyes-Acosta et al. (2012) proposed a Cyclic Heat Dissipation (CHD) method to extrapolate cyclic in-situ signal measurements to thermal steady-state equilibrium without using any extra installations. The CHD method was also used in this study because it: (a) uses the original Granier TDP calibration, thus it can be applied to majority of tree species; (b) was developed in a semi-arid area on savannah-type of trees exposed to NTG; (c) yielded 
good validation results both in the field (Lubczynski et al., 2012) and in laboratory gravimetric-verifications (Reyes-Acosta et al., 2012); and (d) substantially reduces power consumption.

Following the CHD method, the TDPs were always inserted radially into the stems $10 \mathrm{~cm}$ apart and with the heating probe at the upper position. All the probes were adequately protected from rainfall and sun radiation by covering installations with aluminium shields. The sensors were checked weekly and reinstalled every 4 weeks or earlier if malfunctioned. Once the NTG bias was confirmed as significant, we acquired cyclic power schema (CPS) data in 10 trees per species from June to September in 2009 and 2010, all at the Northern azimuth TDP installations and all at the $1.30 \mathrm{~m}$ height above the ground surface. The CPS measurements consisted of 7.5 min powered transients (ON) and 7.5 min unpowered transients (OFF). In each transient, the data was sampled and stored with $30 \mathrm{~s}$ interval (Campbell CR1000 for Q.i. and Skye DataHog2 for Q.p.). This scheme provided 4 measurement cycles (i.e. 4 measurement points) per hour (15 min each). The lengths of the ON/OFF transients $(7.5 \mathrm{~min})$ were defined by finding a minimum number of samples per transient required to adequately extrapolate the signal to thermal steady-state equilibrium (Figure 3.3). The ON-OFF transients were implemented by using a custom-specific power-relay regulator (UP Gmbh, Germany) designed to count pre-programmed switching power intervals controlled by the logger.
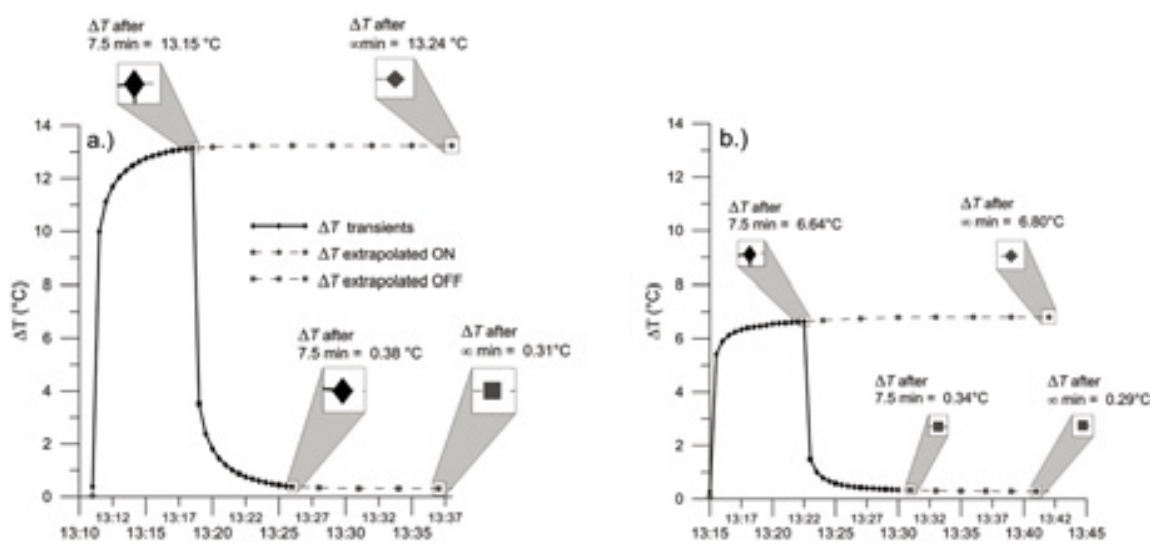

Figure 3.3: Randomly selected transients (bold lines), modelled (extrapolated dashed lines) to thermal equilibrium in: (a) Q.i. and (b) Q.p. Zoomed areas emphasize the last measured $\Delta T$ at $7.5 \mathrm{~min}$ and the extrapolated to thermal equilibrium $\Delta T$.

The objective of the CHD correction is to obtain an un-biased by NTG $\Delta T$ signal $\left(\Delta T_{O N_{i}}^{C}\right)$. This is done for each cycle as described in section 2.2.5 of the dissertation. Finally, after calculating the data, the standard TDP calibration was used to calculate sap flux density $\left(J_{p}\right.$ in $\mathrm{cm}^{3} \mathrm{~cm}^{-2}$ hour $^{-1}$ ) according to the measured temperature difference ( in deg.) 
between the two TDP probes, as in the Equation (2.1) and (2.2) (Granier, 1985). In these equations $\Delta T_{\max }$ is used as a reference $\Delta T$ corresponding with no-flow conditions.

\subsubsection{Step 2: Night flow $\Delta T_{\max }$ correction}

The accuracy of the TDP method is highly dependent on the presence of no-flow conditions expected to occur in the night time but in practice not always present. Additionally the TDP method cannot uniquely discriminate whether such no-flow conditions were present or not. Lu et al. (2004) proposed to assign $\Delta T_{\max }$ for TDP sensors by either calculating a 7-10 days moving average from daily $\Delta T_{\max }$ measurements, or by using a double regression of continuous $\Delta T_{\max }$ measurements in case of drift (due to water stress or other factors). These procedures are useful to find episodes of zero night flow (Lu et al., 2004) but unfortunately were not applicable in our case, because in all Q.p. trees and some Q.i. trees, the night flow did not show any zero-flow as revealed by the HFD measurements (see below).

Regalado and Ritter (2007) introduced another method to account for non-zero $\Delta T_{\max }$ based on a re-arrangement of the Granier's equation, assuming that $J_{p}$ from standard TDP is an actual estimation of transpiration and depends on potential evapotranspiration (PET). However, we decided not to use that method because: (a) $J_{p}$ from standard TDP cannot be assumed to be equal to transpiration when the trees have a sapwood $>2 \mathrm{~cm}$, without accounting for radial-azimuthal variability; (b) the stomata-closure dynamics can reduce $J_{p}$ when the PET is high in semi-arid conditions (Mediavilla and Escudero, 2003), and it is unknown how this affects the linear relation between PET and $\Delta T$ used for the $\Delta T_{\max }$ determination.

Alternatively, $\Delta T_{\max }$ corresponding with zero flow can be recorded by cutting a large enough branch or by carving out a wide enough portion of a stem, and continuously monitoring the $T$ data until it stabilizes (Williams et al., 2004). The same procedure has been applied to root-flow measurements (Nadezhdina et al., 2006b). However, cutting a branch provides wood material that may have different thermal properties than sapwood at the TDP probe insertion, and carving out a portion of the stem is cumbersome and destructive, thus not applicable for protected trees like $Q . i$.

In this study, we used the HFD as a reference for night-flow assessment and optimized Equations (2.1) and (2.2) to fit that reference using a parameter-optimisation algorithm called Shuffled Complex Evolution Metropolis or SCEM-UA (Vrugt et al., 2003b). The SCEM-UA applies controlled random search, competitive evolution, and complex shuffling in order to find the best posterior target distribution of assigned parameters (i.e. best set of values for considered parameters to fit a specific target). In this study $\Delta T_{\max }$ was set as the parameter to be optimized in Granier's equation (Equation (2.2)). A random set of $\Delta T_{\max }$ values (within a logic likely range) was assigned to obtain $J_{p}$ estimates comparable to the HFD 
reference measurements. After a short number of iterations, the $\Delta T_{\max }$ value that yielded the best fit was selected to calculate the corrected $J_{p}$.

\subsubsection{Step 3: Radial and azimuthal TDP correction}

The HFD method considered in this study as reference, determines $J_{p}$ based on the spatial deformation of a heat-field around a linear heater (placed tangentially in the sapwood of the studied tree), represented by the ratio of the measured temperature differences symmetrically $\left(\delta T_{s y m}\right)$ and asymmetrically ( $\delta T_{\text {asym }}$ ) (Nadezhdina et al., 1998, 2002, 2006a, 2012).

In our measurements we used the HFD sensor customized for the SL5 SMART digital logger (ICT International, Armidale, NSW, Australia). This sensor uses one heating needle of $11 \mathrm{~cm}$ and three $10 \mathrm{~cm}$ probes to measure sapwood temperature data. Each probe is equipped with 8 equidistant thermistors, starting at $5 \mathrm{~mm}$ from the base of the probes and spaced every $10 \mathrm{~mm}$. The measurements of radial variability of sap flux density $\left(J_{p-i}\right)$ were carried out on 10 trees per tree species, during 4 to 5 days between August and September in 2009 and between June and September in 2010, always at the Northern azimuth installations.

The dry season, long term temporal variability of the sap-flux density $\left(J_{p-m t h}\right)$ was additionally monitored on two trees of Q.i. and two trees of Q.p., from June to September 2010 for at least 10 days each month also at the Northern azimuth. The measurements of azimuthal variability of sap flux density $\left(J_{p-a z}\right)$ were carried out at 4 azimuth installations (North, West, East and South), for 2-3 days in the same 4 trees (2 Q.i. and 2 Q.p.) as the long term measurements.

Due to the limited availability of HFD sensors, the long term and the $J_{p-a z}$ measurements were performed only on the trees with DBH size category $20-50 \mathrm{~cm}$, which contributed the most to the population variability of both species (Figure 3.2a). All the HFD measurements were sampled every 30 seconds and stored as averages in 10 min intervals using a SL5 Smart logger (ICT International, Armidale, NSW, Australia). For HFD data processing we used the following equation proposed by Nadezhdina et al. (2012) to calculate sap flux density in each radial position:

$$
J_{p-i}=3600 D \times\left(K_{i}+\delta T_{s a-i}\right) \delta T_{\text {asym }-i}^{-1} Z_{a x} Z_{t g}^{-1}
$$

where $\mathrm{D}$ is the thermal diffusivity of fresh wood $\left(\mathrm{cm}^{2} \mathrm{~s}^{-1}\right) ; K_{i}$ is the dimensionless value representing zero flow conditions for the temperature gradients $\delta T_{s a-i}$ and $\delta T_{a s y m-i} ; \delta T_{s a-i}$ is the temperature difference between the symmetrical and asymmetrical needle at position $i ; \delta T_{\text {asym-i }}$ is the asymmetric temperature difference at position ${ }_{i} ; Z_{a x}$ is the axial distance between the upper end of the asymmetrical thermocouple and the heater; $Z_{t g}$ is the distance between the tangential needle and the heater (standard distances for the ICT HFD sensors are $1.5 \mathrm{~cm}$ and 0.5 
$\mathrm{cm}) ; K_{i}$ was determined empirically from the difference between $\delta T_{s y m-i}$ and $\delta T_{\text {asym-i }}$, and the relation with their ratio $\left(\delta T_{s y m-i} / \delta T_{s y m-i}\right)$ (Nadezhdina et al., 2006a, 2012).

The thermal diffusivity $(D)$ is a key parameter for calculating $J_{p}$ using the HFD method as per Equation (3.1). As a rule of thumb, $D$ is defined as $0.0025 \mathrm{~cm}^{2} \mathrm{~s}^{-1}$ based on previous conifer tree studies (Marshall, 1958). In order to improve the HFD $J_{p}$ accuracy and to account for differences between $Q . i$. and $Q . p$. we experimentally defined $D$ for both species from green and dry volume/weight measurements following the procedures proposed by Vandegehuchte and Steppe (2012). We took three fresh core samples per tree: in the morning, at the midday and in the afternoon. Immediately after, samples were stored in pre-weighted sealed glass vials to avoid evaporation. Back in the laboratory, samples were weighed and green-volume calculated using the length of the sample and the standardized diameter of the Pressler increment corer (5 $\mathrm{mm}$ diameter). Afterwards, the wood samples were oven-dried at $60^{\circ} \mathrm{C}$ until constant weight was obtained.

A final issue regarding the use of the HFD technique as reference method in the proposed step-wise optimisation is the eventual sensitivity of the HFD technique to NTG bias. To our knowledge, impact of NTG on the HFD signal has not been proved in field measurements yet. This concern is relevant because the HFD sensor relies on the thermal dissipation principle, that in the TDP method proved to be highly sensitive to the NTG bias (Do and Rocheteau, 2002a; Lu et al., 2004; Lubczynski et al., 2012; Reyes-Acosta et al., 2012). However, the HFD $J_{p-i}$ depends on a ratio of temperature measurements taken by the 3 HFD probes in close distance from each other (axial probes $1.5 \mathrm{~cm}$ and tangential robe $0.5 \mathrm{~cm}$ ), thus theoretically $J_{p}$ is not affected by NTG (Nadezhdina et al., 2008). Preliminary tests performed in collaboration with the Laboratory of Plant Ecology of the University of Ghent, (unpublished) demonstrated that the HFD measurements could be NTG-biased under: i) very strong NTG impact; ii) fast-changing NTGs; and iii) very low $J_{p}$. However, the HFD bias was much lower than the TDP bias in comparable conditions and besides the laboratory enforced NTG was significantly stronger and changed faster than the NTG measured in the field. Nevertheless, anticipating that the NTG could affect the HFD, we minimized this potential effect by: (1) insulating the HFD sensors during the field campaigns with a kneading-paste used for water-isolation and aluminium shielding; (2) making sure that the heat field was well established by using the highest advisable voltage and by assuring that $\delta T_{\text {asym-i }}$ ratio was not $<1{ }^{\circ} \mathrm{C}$; and finally (3) monitoring the data to filter out over-shoots resembling NTG bias patterns observed in the laboratory.

In order to correct radially and azimuthally the TDP measurements, first we had to define a standard HFD profile $\left(J_{p-s t d}\right)$ representing radial and azimuthal $J_{p}$ variability across different tree sizes. To account for radial $J_{p}$ variability across different tree sizes $\left(J_{p-i-t r e e s}\right)$ we averaged $J_{p}$ over the 10 tree profiles per species (all northern azimuth) weighted by the number of sampled trees in each DBH size category, as shown in 
Table 3.1. To account for the azimuthal variability $\left(J_{p-a z}\right)$ we averaged the $J_{p}$ over the 4 azimuthal measurements per tree and between the selected trees of the same size category. The final reference $J_{p-s t d}$ applied for the corrections of Step 2 and Step 3, was derived as an average of $J_{p-i \text {-trees }}$ and $J_{p-a z}$.

As the standard TDP has only $2 \mathrm{~cm}$ length, various approaches have been proposed to adjust TDP data for $J_{p-i}$ radial variability based on: (1) proportionality corrections (Tang et al., 2006; Ewers et al., 2007; Poyatos et al., 2007; Steppe et al., 2010); (2) fitting polynomial functions (Bernier et al., 2002; Ford et al., 2004); (3) fitting a Gaussian distribution (Ford et al., 2004); and more recently (4) using Bayesian distributions (Bernier et al., 2002; Caylor and Dragoni, 2009; Dragoni et al., 2009). In our study we decided to use the proportionality correction method of Steppe et al. (2010) because (1) it is simple to process; (2) it can handle complex patterns of $J_{p}$ radial profiles such as non- uniform $J_{p}$ decrease towards the hardwood or large $J_{p}$ in deep sapwood layers; and (3) it accounts for the temporal variability of the radial profiles. This method scales the $2 \mathrm{~cm}$ deep $J_{p}$ TDP measurements to the $J_{p-s t d}$ profile, by establishing ratios between the two outer HFD $J_{p-s t d}$ measurements, corresponding with the external $2 \mathrm{~cm}$ radial profile, and the $J_{p-s t d}$ at each of the other 6 sapwood depths. The obtained proportionally ratios were then used to extrapolate the TDP $J_{p}$ towards other depths corresponding with the HFD $J_{p-s t d}$.

\subsubsection{Criteria to assess the optimized TDP measurements}

We calculated the Root Mean Squared Error (RMSE) in steps 1 and 2 as the main accuracy criterion of the agreement between the corrected TDP $J_{p}$ and the reference HFD $J_{p}$, the latter taken as the average of the two outermost measuring points in the sapwood $\left(H F D_{2 \mathrm{~cm}}\right)$. In step 3, the RMSE was defined by comparing the TDP final $_{\text {with }}$ the $J_{p}$ average of the $\mathrm{HFD}_{\text {ref }}$ for the considered sapwood depths.

\subsection{Results and Discussion}

\subsubsection{Dry-season environmental conditions and selected days for comparison}

Meteorological data of the Trabadillo station showed that the study area can be classified as water limited environment (WLE) following Abrahams and Parsons (1994) and Guswa et al. (2004). In the dry seasons of June to September 2009 and 2010, mean PET largely exceeded mean rainfall $(\approx 4$ mm day ${ }^{-1}$ PET $>>\approx 0.04 \mathrm{~mm} \mathrm{day}^{-1}$ of rain). In addition, mid-day dry season VPD (3.5-4.8 kPa), soil-moisture (3- 9\%) and soil matric potential (-2 MPa at 25-50 cm depth) suggested that trees were exposed to low water availability during the monitored period. Besides, the mean daily maximum of incoming short wave solar-radiation for clear-sky days 
was between 700 and $1000 \mathrm{~W} \mathrm{~m}^{-2}$, which was at the upper level of the possible yearly maxima of incoming radiation for this area. The daily maxima of air temperatures were $>30^{\circ} \mathrm{C}$ while the relative humidity minima were $<30 \%$. These climatic conditions implied plant droughtstress during the monitored period.

\subsubsection{Tree population structures of $Q . i$. and $Q . p$.}

Out of the measured biometric characteristics the DBH provided the best information to define the population structure for both species (Table 3.2). The population structure for Q.i. (Figure 3.2a) was predominately constituted by trees with DBH between 20 and $50 \mathrm{~cm}$, and a few trees of high DBH $(70-130 \mathrm{~cm})$. In contrast, the population of Q.p. (Figure $3.2 b)$ was uniformly represented by trees of small to medium DBH (20$40 \mathrm{~cm}$ ), with some large trees with DBH up to $100 \mathrm{~cm}$. The different population structures of $Q . i$. and Q.p. are mainly due to their specific stem wood anatomy: Q.i. is an evergreen oak that grows slowly but can reach considerable size due to the hardness of its wood and is considered diffuse semi-ring-porous (Hernández-Santana et al., 2008b); Q.p., is a deciduous oak (Hernández-Santana et al., 2008b) that grows mainly during summer and is characterized by ring-porous wood of low density that frequently causes the collapse of large trees under their own weight.

The statistical significance of the population structure defined by the survey results for both species was demonstrated by the cumulative frequency curves (Figure 3.2b). The curves stabilized from $80 \mathrm{~cm}$ DBH towards the largest DBH sizes which explained $>90 \%$ of the DBH variability, thus indicating that the DBH size variability was significantly covered for the populations. This significance was maintained for the chosen samples of sap flow measurement (10 trees per species), by using stratified sampling. This procedure guaranteed that the acquired sap flow data were representative and conclusive for the tree population variability. Similar methodologies have been successfully used in other open woodlands (Poyatos et al., 2007) and in closed forests (Čermák et al., 2004).

\subsubsection{Thermal diffusivity of Q.i. and Q.p.}

The average thermal diffusivity $(D)$ was $0.00259 \mathrm{~cm}^{2} \mathrm{~s}^{-1}$ for $Q . i$. and $0.00256 \mathrm{~cm}^{2} \mathrm{~s}^{-1}$ for $Q . p$. (Table 3.2). The $D$ values were used as inputs for Equation (3.1) in the sap flow tool software used for calculating $J_{p}$ in the HFD method. Both values were slightly higher than the commonly used $D$ values for pine trees $\left(0.0025 \mathrm{~cm}^{2} \mathrm{~s}^{-1}\right)$ provided by Marshall (1958), thus increasing the $J_{p}$ magnitudes obtained from the HFD. To our knowledge, these are the first reported $D$ values for Q.i. and Q.p. 
Table 3.2: Biometric characteristics of the 10 sampled trees of Q.i., and 10 sampled trees of Q.p. in the Sardón catchment 2009-2010.

\begin{tabular}{|c|c|c|c|c|c|c|c|}
\hline $\begin{array}{l}\text { Statistics } \\
\text { Q.i. }\end{array}$ & $\begin{array}{l}\text { Stem } \\
\text { DBH at } \\
1.30 \mathrm{~m}\end{array}$ & $\begin{array}{l}\text { Tree } \\
\text { Height }\end{array}$ & $\begin{array}{l}\text { Xylem } \\
\text { depth } \\
\text { dyed } \\
\text { core }\end{array}$ & $\begin{array}{l}\text { Xylem } \\
\text { depth } \\
\text { HFD } \\
\left(l_{s}\right) \\
\end{array}$ & $\begin{array}{l}\text { Sapwood } \\
\text { area } \\
\text { corer }\left(A_{x}\right)\end{array}$ & $\begin{array}{l}\text { Sapwood } \\
\text { area } \\
\text { HFD }\left(A_{x}\right)\end{array}$ & Diffusivity \\
\hline & $\mathrm{cm}$ & $\mathrm{m}$ & $\mathrm{cm}$ & $\mathrm{cm}$ & $\mathrm{cm}^{2}$ & $\mathrm{~cm}^{2}$ & $\mathrm{~cm}^{2} \mathrm{~s}^{-1}$ \\
\hline AVERAGE & 38.19 & 7.68 & 7.28 & 6.89 & 536.12 & 590.1 & 0.00259 \\
\hline MIN & 17.00 & 5.46 & 5.00 & 5.00 & 150.72 & 153.08 & 0.00253 \\
\hline MAX & 97.08 & 9.45 & 12.00 & 7.50 & 1398.56 & 1787.51 & 0.00265 \\
\hline MEDIAN & 28.25 & 8.02 & 7.15 & 7.50 & 474.14 & 365.03 & 0.00259 \\
\hline STDEV & 25.57 & 1.60 & 2.10 & 0.86 & 387.75 & 526.12 & 0.00006 \\
\hline $\begin{array}{l}\text { Statistics } \\
\text { Q.i. }\end{array}$ & $\begin{array}{l}\text { Stem } \\
\text { DBH at } \\
1.30 \mathrm{~m}\end{array}$ & $\begin{array}{l}\text { Tree } \\
\text { Height }\end{array}$ & $\begin{array}{l}\text { Xylem } \\
\text { depth } \\
\text { dyed } \\
\text { core }\end{array}$ & $\begin{array}{l}\text { Xylem } \\
\text { depth } \\
\text { HFD } \\
\left(l_{s}\right)\end{array}$ & $\begin{array}{l}\text { Sapwood } \\
\text { area } \\
\operatorname{corer}\left(A_{x}\right)\end{array}$ & $\begin{array}{l}\text { Sapwood } \\
\text { area } \\
\operatorname{HFD}\left(A_{x}\right)\end{array}$ & Diffusivity \\
\hline & $\mathrm{cm}$ & $\mathrm{m}$ & $\mathrm{cm}$ & $\mathrm{cm}$ & $\mathrm{cm}^{2}$ & $\mathrm{~cm}^{2}$ & $\mathrm{~cm}^{2} \mathrm{~s}^{-1}$ \\
\hline AVERAGE & 31.50 & 8.58 & 3.11 & 4.13 & 267.87 & 294.26 & 0.00256 \\
\hline MIN & 15.70 & 6.10 & 2.00 & 3.50 & 84.78 & 115.87 & 0.00251 \\
\hline MAX & 59.36 & 10.10 & 5.50 & 4.50 & 878.35 & 580.93 & 0.00261 \\
\hline MEDIAN & 32.30 & 9.13 & 2.70 & 4.50 & 172.79 & 284.64 & 0.00260 \\
\hline STDEV & 13.83 & 1.63 & 1.13 & 0.52 & 258.72 & 151.63 & 0.00005 \\
\hline
\end{tabular}

\subsubsection{First step: Natural temperature gradients bias correction with the CHD method}

The NTG measurements presented in Figure 3.4 and 3.5 are referenced to the Spanish summer time, GMT+1, which is approximately 2.5 hours ahead of the geographical ("solar") time. Following that setup, sunrise and sunset in June 2009 were scheduled at 6:52 and 22:01 in the study area. The positive NTG in Figure 3 and 4 indicates that the upper probe (normally heated except during NTG measurements) had a higher temperature than the bottom reference probe, while the negative that the upper probe had a lower temperature than the bottom probe. As a first approximation Do and Rocheteau (2002a) considered an NTG outside the range $-0.20 \mathrm{C}$ to $+0.20{ }^{\circ} \mathrm{C}$ as non-negligible, hence one significant bias to be corrected. In the majority of our measurements the recorded NTG was outside that range.

The measured NTG signal presented in Figure 3.4 and 3.5 varied between: (a) the 2 species; (b) the 4 different azimuthal installations (North, East, West and South); and (c) the 3 heights of the installations in the stem (Bottom, Middle and Upper). The NTG of Q.i. was consistently 


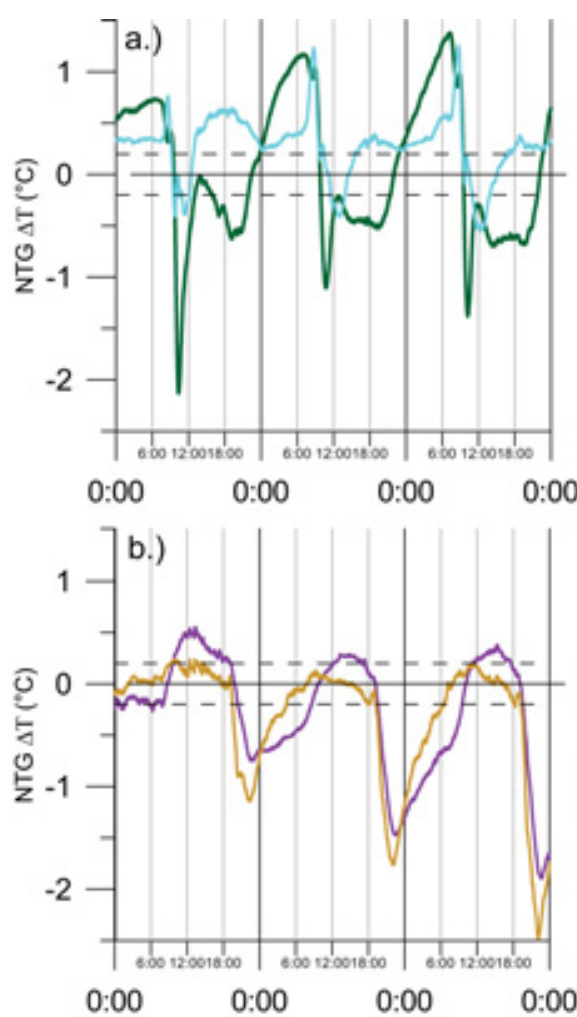

Quercus ilex

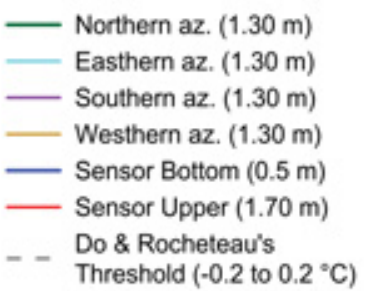

Figure 3.4: Daily variations of NTG acquired by unpowered TDP at different azimuths and different tree heights in Q.i. (a) North-easterly pattern; (b) South-westerly pattern and (c) Bottom-Upper patterns at the Northern azimuth. The time axis follows GMT+1 Spanish summer time for all figures.

higher and with larger variations (e.g. $-2.47 t^{\circ} \mathrm{C}$ o $1.38 a^{\circ} \mathrm{C}$ t Northern azimuth) than of Q.p. (e.g. $-0.14 t^{\circ} \mathrm{C}$ o $0.19 a^{\circ} \mathrm{C}$ t Northern azimuth) in all TDP installations (Figure 3.4 and 3.5), despite the same climatic conditions of the experiment. The NTG differences between the two species are therefore likely constrained by different thermal properties of xylem.

The azimuthal measurements of NTG in Q.i. show two opposite patterns (Figure 3.4): the North-Easterly pattern and the South-Westerly pattern. The North-Easterly pattern, indicated positive NTG at the end of each day and through the entire night, rising towards the maximum $(>+1$ ${ }^{\circ} \mathrm{C}$ ) before sunrise. After sunrise, we observed a sharp decrease towards negative daytime NTG. The South-Westerly pattern indicated NTG sharply decreasing at the end of the day towards a negative minimum $\left(<-1{ }^{\circ} \mathrm{C}\right)$ reached before sunset. From this point the NTG steadily increased over night towards a positive maximum in the following day. The azimuthal NTG pattern of Q.p. (Figure 3.5) was not as distinct as the one of Q.i., 


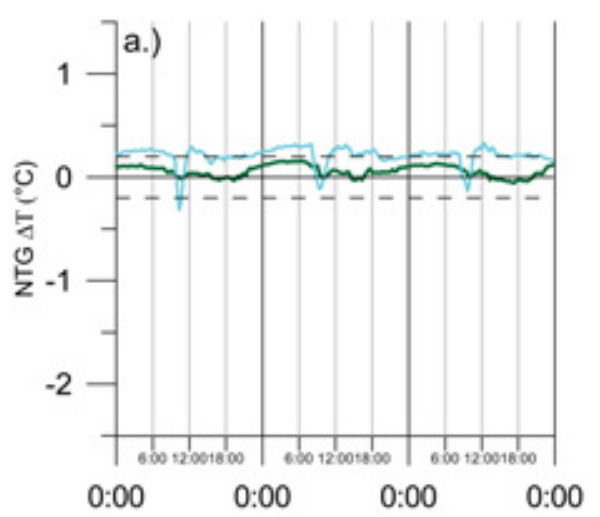

\section{Quercus pyrenaica}

Northern az. $(1.30 \mathrm{~m})$

Eastern az. (1.30 m)

Southern az. $(1.30 \mathrm{~m})$

Western az. (1.30 m)

Sensor Bottom $(0.5 \mathrm{~m})$

Sensor Upper (2.00 m)

Do \& Rocheteau's

Threshold $\left(-0.2\right.$ to $\left.0.2{ }^{\circ} \mathrm{C}\right)$
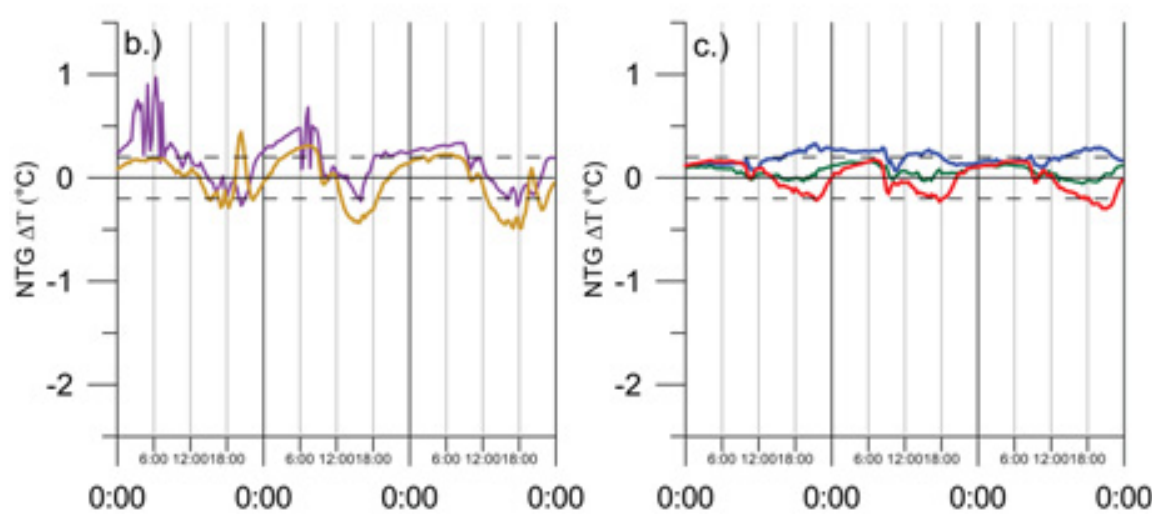

Figure 3.5: Daily variations of NTG acquired by unpowered TDP at different azimuths and different tree heights in Q.p. (a) North-easterly pattern; (b) South-westerly pattern and (c) Bottom-Upper patterns at the Northern side. The time axis follows GMT+1 Spanish summer time for all figures.

but followed a similar Northern-Easterly and South-Westerly tendency. The NTG at the Northern and Eastern azimuth was generally positive around $0.3{ }^{\circ} \mathrm{C}$ with an evident negative morning peak of $-0.3{ }^{\circ} \mathrm{C}$, whereas at the Southern and Western azimuth the NTG was generally positive after sunrise and negative in the afternoon. Interestingly, most of the observed NTG patterns at the Northern azimuth for both species well resembled the typical NTG patterns (positive during night and negative during days) described in previous studies that were carried out under semi-arid conditions (Do and Rocheteau, 2002a; Lubczynski et al., 2012), whereas the patterns of the other azimuths were different either in shape or in shape and phase. Unfortunately, our NTG measurements did not provide enough evidence to generalize azimuthal NTG observations, but proved large azimuthal NTG variability. Thus, we advise to be cautious with the generally suggested installations at the Northern azimuth (Lu et al., 2004) if NTG is not reliably corrected.

The NTG measurements also indicated dependence on the height of 
the sensor installation. For Q.i. (Figure 3.4c), the Bottom $(0.5 \mathrm{~m})$, the Middle $(1.3 \mathrm{~m})$ and the Upper $(1.7 \mathrm{~m})$ NTG measurements at the Northern azimuth, showed significant NTG that ranged between $-2.1^{\circ} \mathrm{C}$ and $1.4^{\circ} \mathrm{C}$. The Bottom installation had an opposite phase as compared to the Middle and the Upper installations and showed a negative or close to zero ${ }^{\circ} \mathrm{C}$ NTG in the morning and a positive during afternoon. The negative morning peak of the Bottom installation was reached $\approx 3$ hours earlier than the negative peaks of the Middle and the Upper installations. For Q.p. (Figure 3.5c), the NTG patterns (measured at the Northern azimuth) at the Bottom $(0.5 \mathrm{~m})$, Middle $(1.3 \mathrm{~m})$ and Upper $(2.0 \mathrm{~m})$ positions were similar to those of Q.i., though less distinct and with NTG ranging between $-0.3^{\circ} \mathrm{C}$ and $0.33^{\circ} \mathrm{C}$ (Figure 3.5).

NTG patterns and magnitudes are very complex, because of their spatio-temporal variability and many factors influencing them (e.g. solar inclination with respect to installation position, heterogeneity of the wood, etc.). Therefore, attempting to explain the NTG patterns according to the species, azimuthal direction, installation height and even sapwood depth is very difficult. However, considering the availability of the in-situ NTG signal correction methods that allow to remove the NTG "noise" from the biased signal, such as CHD (Lubczynski et al., 2012; ReyesAcosta et al., 2012) and TTD (Do and Rocheteau, 2002a,b; Isarangkool Na Ayutthaya et al., 2010), explaining the observed NTG patterns is of less relevance with respect to quantifying sap flow.

As the significant NTG bias was detected while using TDP sensors, we applied the CHD method on both species. From preliminary tests we found that $7.5 \mathrm{~min}$ transient OFF and $7.5 \mathrm{~min}$ transient ON were long enough to fit well the model to the data and short enough to avoid undesired signal drift (Lubczynski et al., 2012). Next, through the leastsquared optimisation of the modelled ON-OFF cycles, we found that the obtained $A_{1} A_{2} A_{3}$ and $T_{3}$ parameters were species-specific, i.e. different for $Q . i$. and Q.p., even though the environmental conditions were the same (Table 3.3).

Table 3.3: Min-max range of the CHD fitting parameters for Q.i. and Q.p.

\begin{tabular}{|c|c|c|c|c|c|c|c|c|c|c|}
\hline & \multicolumn{2}{|c|}{$\begin{array}{c}A_{1} \\
{\left[{ }^{\circ} \mathrm{C}\right]}\end{array}$} & \multicolumn{2}{|c|}{$\begin{array}{c}A_{2} \\
{\left[{ }^{\circ} \mathrm{C}\right]}\end{array}$} & \multicolumn{2}{|c|}{$\begin{array}{c}A_{3} \\
{\left[{ }^{\circ} \mathrm{C}\right]}\end{array}$} & \multirow{2}{*}{$\begin{array}{l}T_{1} \\
{[\mathrm{sec}]} \\
\text { Const. }\end{array}$} & \multirow{2}{*}{$\begin{array}{l}T_{2} \\
{[\mathrm{sec}]} \\
\text { Const. }\end{array}$} & \multicolumn{2}{|c|}{$\begin{array}{c}T_{3} \\
{[\mathrm{sec}]}\end{array}$} \\
\hline & ON & OFF & ON & OFF & ON & OFF & & & ON & OFF \\
\hline Q.i. & $8.7-10.5$ & 8.9-11.1 & $0.5-2.5$ & $0.5-1.5$ & $1.8-4.3$ & $2.0-3.4$ & 10.5 & 45.5 & $100-300$ & $84-204$ \\
\hline Q.p. & $5.2-6.5$ & $5.2-6.0$ & $0.9-1.9$ & $1.3-1.7$ & $0.6-1.9$ & $0.6-1.7$ & 7.5 & 45 & $127.5-331.5$ & $133.8-416.9$ \\
\hline
\end{tabular}

Figure 3.6 illustrates the overall change in the TDP $J_{p}$ after applying CHD method in Step 1 by comparing "Non corrected" and "After Step 1 " TDP measurements with $H F D_{2 c m}$ measurements. The RMSE (in $\mathrm{cm}^{3}$ $\mathrm{cm}^{-2}$ hour $^{-1}$ ) between standard non-corrected TDP (TDP $\left.{ }_{s t d}\right)$ and $J_{p}$ from $H F D_{2 \mathrm{~cm}}$ across DBH sizes (Figure 3.6a and fig:RMSEBarsb), ranged from 1.1 to 7.9 for Q.i. (average $=3.7$ and $\mathrm{SD}=2.2$ ) and from 1.9 to 5.1 for Q.p. (average $=2.8$ and $\mathrm{SD}=1.9$ ). After removing the NTG bias (Step 1 in 
Figure 3.6a and 53.6b), the RMSE between $J_{p}$ from TDP measurements corrected for NTG $\left(\mathrm{TDP}_{N T G-\text { free }}\right)$ and $J_{p} H F D_{2 \mathrm{~cm}}$ across DBH sizes was between $0.9-2.4$ (average $=1.0$ and $\mathrm{SD}=0.5$ ) for $Q . i$. and $1.9-6.1$ for Q.p. (average $=4.0$ and $\mathrm{SD}=1.3$ ). In general the RMSE for Q.i. was reduced but we noted a slight increment of the RMSE for three $Q . i$. trees with $\mathrm{DBH}=37,50$ and $97.1 \mathrm{~cm}$ after NTG correction (Figure 3.7). This increment was caused by a significant effect of the night flow on the $\Delta T_{\text {max }}$ that generated an underestimation of $J_{p}$. In the case of Q.p. the RMSE increased after NTG correction in all trees, with exception of two trees with $\mathrm{DBH}=14.7$ and $36.6 \mathrm{~cm}$ (Figure 3.8). The reason for an opposite effect on the RMSE in Q.p. as compared to the RMSE of Q.i., was the influence of the night flow on the $\Delta T_{\max }$ that was much more significant in Q.p. than in Q.i. trees (see next section for more details on night flow). Despite the effect of the night flow, the NTG correction not only adjusted the magnitude of $J_{p} \mathrm{TDP}_{N T G-f r e e}$ but also smoothed its patterns for all Q.i. and Q.p. trees (Figure 3.7 and 3.8).
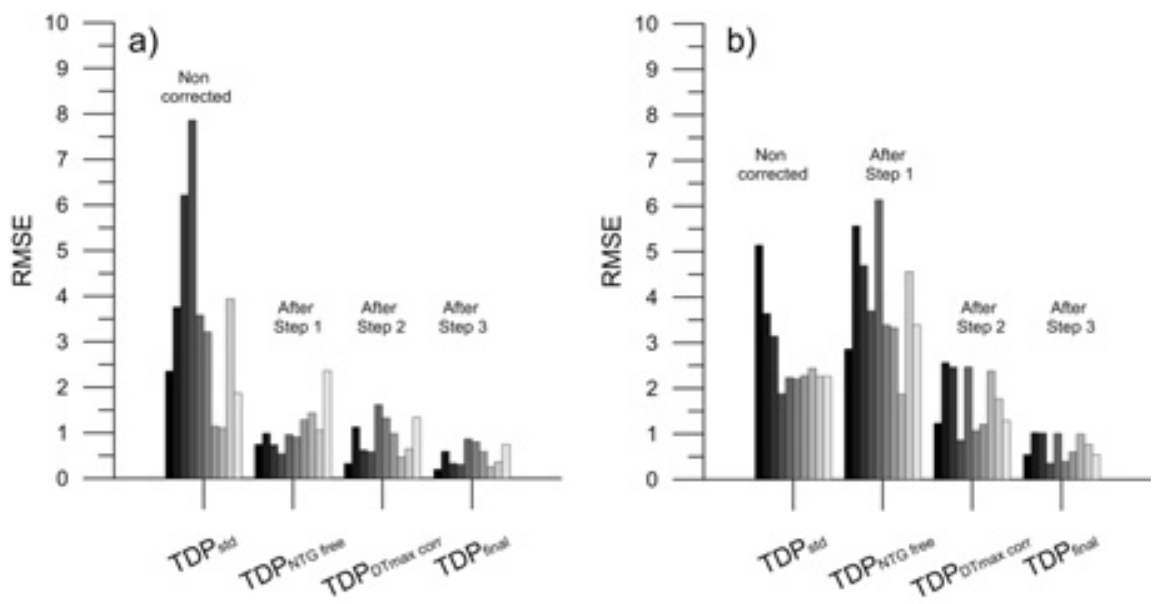

Figure 3.6: $J_{p}$ root mean squared errors (RMSE) between: (1) noncorrected data $\left(\mathrm{TDP}_{s t d}\right)$ and $H F D_{2 c m}$; (2) $\mathrm{TDP}_{N T G-\text { free }}$ and $H F D_{2 c m}$ (step 1) (3) $\mathrm{TDP}_{\Delta T \text { max }- \text { corr }}$ and $H F D_{2 c m}$ (step 2); (4) $\mathrm{TDP}_{\text {final }}$ and $\mathrm{HFD}_{\text {ref }}$ (step 3) for: (a) Q.i. and (b) Q.p. Each bar shows the RMSE for a single tree measured during 2010; the darker gradient of the bars represents smaller DBH and lighter larger DBH.

By comparing $J_{p} \mathrm{TDP}_{N T G-f r e e}$ with $J_{p} \mathrm{TDP}_{s t d}$ (Figures 3.7 and 3.8), from the first step of the optimization, we could observe that despite the NTG of Q.i. (Figure 3.4) was significantly larger than that of Q.p. (Figure 3.5), the CHD correction of both Q.i. and Q.p. signal resulted in a comparable reduction of $J_{p}$, on average $64 \%$ for Q.i. (Figure 3.7) and $55 \%$ for Q.p. (Figure 3.8). This was likely the case because the raw $\Delta T$ signal of Q.p. (Figure 3.3) was lower $\left(\approx 7-10^{\circ} \mathrm{C}\right)$ than that of $Q . i$. $\left(\approx 11-13^{\circ} \mathrm{C}\right)$, hence the NTG bias in the case of Q.p. (characterized by significantly lower denominator in the Equation (2.2)) had proportionally 
larger impact on the calculated $J_{p}$ than in the case of Q.i., for an in-depth discussion, the reader is referred to (Lubczynski et al., 2012).
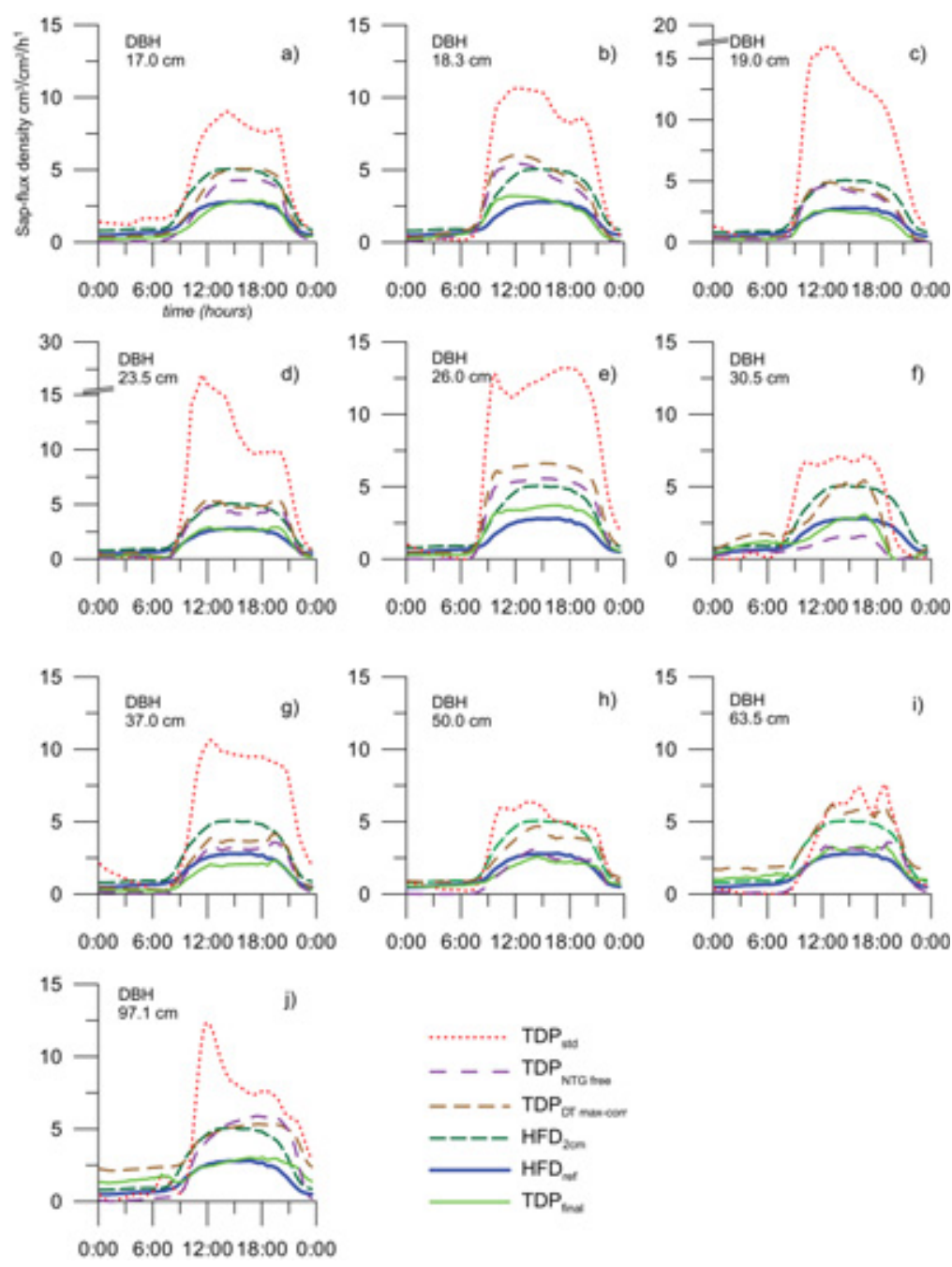

Figure 3.7: Step-wise optimisation of TDP $J_{p}$ measurements using HFD as reference, for 10 selected Q.i. trees $(\mathrm{a}-\mathrm{j})$ : $\mathrm{TDP}_{s t} \mathrm{~d}^{-}$uncorrected meas-

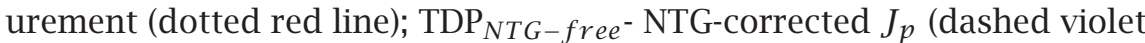

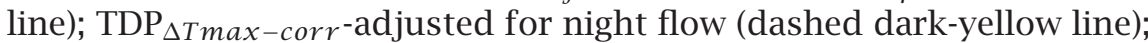
$\mathrm{TDP}_{\text {final }}$ - corrected for azimuthal and radial variability $J_{p}$ (light-green line); $H F D_{2 c m}$-reference for the $2 \mathrm{~cm}$ long TDP sensors (dashed darkgreen line); and $\mathrm{HFD}_{r e f^{-}}$(bold blue line) radial-azimuthal $J_{p}$ reference (the time axis follows GMT+1 Spanish summer time). 


\subsubsection{Second step: Night flow determination and correction}

The second step required an external reference method to define $\Delta T_{\max }$ under continuous night flow for the TDP method, which itself cannot handle this problem. In this study, HFD method was used as such reference method.

Continuous upward night flow in Q.i. as measured by HFD was generally negligible, except for 3 of the 10 investigated Q.i. trees with DBH sizes 30.5, 37.0 and $97.1 \mathrm{~cm}$ that had an average night flow of 1.05 $\mathrm{cm}^{3} \mathrm{~cm}^{-2}$ hour $^{-1}(\mathrm{SD}=0.13$ ) (Figures 3.7a). In contrast to Q.i., all 10 Q.p. trees experienced continuous, upward night flow with an average night flow of $3.26 \mathrm{~cm}^{3} \mathrm{~cm}^{-2}$ hour $^{-1}$ ( $\mathrm{SD}=0.09$ ) (Figure 3.8 ). The significantly larger night flow of Q.p. than in Q.i. points at different water-use control mechanisms (i.e. strategies) of the two species. A higher continuous night flow implies that the Q.p. trees have poorer stomatal control and/or are facing higher water stress, whereas $Q . i$. trees have a more efficient mechanism but some are facing water-stress as well. The night flow observed in both species did not show significant correlation with the climatic conditions such as night VPD and night air temperature, indicating that the driving forces behind night flow are not related with night time climatic conditions.

To our knowledge, our results are the first reporting night flow for Q.p. and Q.i. However, Mediavilla and Escudero (2003) pointed out that Q.p. has high stomatal-conductance, low sensitivity to soil-moisture availability and low sensitivity to VPD. Hernández-Santana et al. (2008b), through their observations of sapwood water storage depletion in Q.p., stated that the day-time depletion was related with the high stomatal conductance and the lack of sensibility to water availability as described by Mediavilla and Escudero (2003). Therefore, night flow can be explained as a nocturnal movement of water from the roots to the canopy, driven by the remnant low water potential after day-time water depletion. The Q.i., is an evergreen species with different leaf types and wood structure as compared to Q.p., thus it is difficult to apply the same explanation to the observed night flow of the Q.i. trees indicating night flow. Further research for investigating this phenomenon is required. The verification of the night flow in Q.i. and Q.p. trees have provided further understanding on the hydrological behaviour of the species and should be considered in future studies.

The continuous presence of night flow in the investigated species contradicts the no-flow assumption of $\Delta T_{\max }$ (Granier, 1985), implying failure of the $J_{p}$ assessment according to Equation (2.1) (Lu et al., 2004). Thus, we defined $\Delta T_{\max }$ by optimising Equations (2.1) and (2.2) to follow the night-flow HFD measurements with the SCEM-UA algorithm. The $J_{p}$ with optimised $\Delta T_{\max }\left(\mathrm{TDP}_{\Delta T \max -c o r r}\right)$, when compared to $H F D_{2 \mathrm{~cm}}$, had RMSE ranging from 0.3 to 1.6 for Q.i. (average $=0.9$ and $\mathrm{SD}=0.4$ ) and from 0.9 to 2.6 for Q.p. (average $=1.8$ and $\mathrm{SD}=0.9$ ). In contrast to the NTG correction (step 1), the $\Delta T_{\max }$ correction resulted in an increase of the $J_{p}$ for both species (Figure 3.7 and 3.8). Our results 
confirmed that detecting night flow conditions was crucial to properly define $\Delta T_{\max }$ and more importantly that the step 1 of the optimization (NTG correction) together with step 2 ( $\Delta T_{\max }$ correction) accounted for most of the disagreement between un-corrected $J_{p} \mathrm{TDP}_{s t d}$ and the $J_{p}$ of $H F D_{2 \mathrm{~cm}}$. Therefore, the suggested use of the HFD method as a night-flow reference for the TDP method allows for an appropriate definition of $\Delta T_{\max }$ and quantification of $J_{p}$ using TDP sensors even when the night flow is present.

\subsubsection{Third step: Accounting for radial-azimuthal sap flux density profiles in TDP measurements.}

The third step, like the second, required an external, reference method to quantify radial and azimuthal sap flow in trees with sapwood thicker than $2 \mathrm{~cm}$. This was because the TDP in the standard implementations have $2 \mathrm{~cm}$ long sensors which cannot handle radial and circumferential $J_{p}$ variability of trees with sapwood depth larger than $2 \mathrm{~cm}$. In this study, HFD method is used as such reference method.

The dry season daily averages of the HFD $J_{p-i}$ measurements of Q.i. and Q.p. (averages of 10 trees per species) followed typical "Gaussian" patterns, i.e. with $J_{p-i}$ decreasing with sapwood-depth (Figure 3.9). The comparison of $J_{p-i}$ of Q.i. and Q.p. show that Q.p. experienced significant continuous night flow, not present or negligible in case of $Q . i$. and had significantly higher dry-season water discharge potential than Q.i. mainly thanks to the large $J_{p}$ in the outer section of the sapwood. The larger $J_{p-i}$ in the outermost sections of sapwood in Q.p. than in Q.i., was not in agreement with the measurements of Lubczynski and Gurwin (2005) carried out in the same study area but with standard TDP measurements ( $2 \mathrm{~cm}$ depth). They found that the $J_{p}$ in Q.i. (from 10 randomly selected trees) was two times higher than in Q.p. (from 22 randomly selected trees). The disagreement was likely because Lubczynski and Gurwin (2005) applied standard TDP measurements without any correction as proposed in this study. The comparison to the data of Lubczynski and Gurwin (2005) points at the importance of the proposed TDP optimisation, for accurate $J_{p}$ measurements. Figure 3.10 shows the monthly $J_{p-m t h}$ averages integrated over the 8 HFD sensors of two Q.i. and two Q.p. trees, for cloud-free days during dry season between June and September. The $J_{p-m t h}$ of the dry season 2010 ranged from $\approx 0.5$ to $3.5 \mathrm{~cm}^{3} \mathrm{~cm}^{-2}$ hour ${ }^{-1}$ in Q.i. and from 0.38 to $0.8 \mathrm{~cm}^{3} \mathrm{~cm}^{-2}$ hour ${ }^{-1}$ in Q.p. for the. The exceptionally low $J_{p-m t h}$ values of the two Q.p. trees were the result of unfortunate pruning activities by the owner of the land at our absence. The same two trees indicated much larger HFD $J_{p-m t h}$ rates the year before pruning (September 2009), comparable with typical rates for Q.p. species as presented in Figure 3.9b, i.e. with daily maximum of $5.96 \mathrm{~cm}^{3} \mathrm{~cm}^{-2}$ hour ${ }^{-1}$ and daily mean of $1.78 \mathrm{~cm}^{3} \mathrm{~cm}^{-2}$ hour-1. Despite this data acquisition obstacle, we still consider the presented in Figure $3.10 \mathrm{~b}$ measurements as valuable because they give an indication of the monthly $J_{p-m t h}$ pattern. The $J_{p-m t h}$ of Q.i. did not show any significant 
difference between different months (Figure 3.10a) while in the case of $Q . p$. the $J_{p-m t h}$ of June was significantly lower $(\approx 75 \%)$ than in the following months (Figure $3.10 \mathrm{~b})$. The low temporal $J_{p-m t h}$ variability for Q.i. is justified because $Q . i$. is an evergreen phreatophyte maintaining daily transpiration patterns during the whole season thanks to the access to a continuous water supply by tapping deep water sources when the soil gets too dry, in addition to its water-conservative strategy (Mediavilla and Escudero, 2003; David et al., 2007). These observations are in agreement with stomata conductance measurements, which have demonstrated that Q.i. has a higher sensitivity to VPD (thus lower stomatal conductance) than Q.p. (Mediavilla and Escudero, 2003). Therefore Q.i. has a more conservative water-use behaviour, which is in full agreement with: (a) the obtained $J_{p}$ measurements of this study and (b) the finding of higher night flow for Q.p. as compared to Q.i. In contrast, Q.p. is a deciduous species which during the spring month of June is still in the process of developing new leaves, therefore has less water demand so less $J_{p}$ despite larger unsaturated zone water availability in June than in the following months (when soil moisture is lower). From July until September, when shoots are well developed and the solar radiation is the highest, Q.p. can maintain a quasi-constant water flow because it grows next to main streams of the catchment. In such locations the water table is shallow so the groundwater is easy to tap and also soil-moisture more available than in the off-stream locations where groundwater table is deeper.

Dry season azimuthal profiles of Q.i. and Q.p., obtained by averaging of eight $J_{p}$ measuring points along the HFD probes in two trees per species, showed that there are significant $J_{p}$ differences between the Northern, Southern, Western and Eastern azimuths (Figure 3.11a and 3.11b). The large $J_{p}$ magnitude difference between the two species are because of the exceptionally low value of the Q.p. $J_{p}$ due to the mentioned above pruning of the Q.p. trees (the same trees as for $J_{p-m t h}$ monitoring presented in Figure 3.10). It is likely however that the temporal and azimuthal patterns are kept after pruning which is assumed in the follow up discussion. In both species the highest $J_{p}$ (averaged across all the HFD depths) was measured at the Northern azimuth, i.e. 2.86 $\mathrm{cm}^{3} \mathrm{~cm}^{-2}$ hour ${ }^{-1}$ (mean $=1.69$ and SD $=0.7$ ) for Q.i. and $0.75 \mathrm{~cm}^{3} \mathrm{~cm}^{-2}$ hour $^{-1}$ (mean $=0.53$ and SD $=0.13$ ) for Q.p. The lowest averaged $J_{p}$ was measured at the Western azimuth for both species, i.e. $0.7 \mathrm{~cm}^{3} \mathrm{~cm}^{-2}$ hour $^{-1}\left(\mathrm{SD}=0.31\right.$ ) for Q.i. and $0.43 \mathrm{~cm}^{3} \mathrm{~cm}^{-2}$ hour $^{-1}$ (mean $=0.38$ and $\mathrm{SD}=0.04)$ for Q.p. respectively. Episodes of back-flow were detected in the Western azimuth of Q.i. at 21:00 and in the Western azimuth of Q.p. at 15:30 and at 20:00 (Figure 3.5b). This was probably due to horizontal stem water transfer (Burgess and Bleby, 2006). However, such back-flow episodes were low (max $=\approx 1 \mathrm{~cm}^{3} \mathrm{~cm}^{-2}$ hour ${ }^{-1}$ for $Q . i$. and $\approx 0.15 \mathrm{~cm}^{3}$ $\mathrm{cm}^{-2}$ hour $^{-1}$ for Q.p.) and not detected in any other azimuths. At the Southern azimuth, the $J_{p}$ was lower than at Northern azimuth and very close to the average for the whole tree $\left(1.09 \mathrm{~cm}^{3} \mathrm{~cm}^{-2}\right.$ hour ${ }^{-1}$ for $Q . i$. and $0.44 \mathrm{~cm}^{3} \mathrm{~cm}^{-2}$ hour $^{-1}$ for Q.p.) and followed the typical Gaussian 
pattern. The maximum $J_{p}$ at the Eastern azimuth of $Q . i$. was $0.9 \mathrm{~cm}^{3}$ $\mathrm{cm}^{-2}$ hour ${ }^{-1}$ (mean $=0.7$ and SD $=0.08$ ) and $0.5 \mathrm{~cm}^{3} \mathrm{~cm}^{-2}$ hour ${ }^{-1}$ for Q.p. (mean $=0.4$ and $\mathrm{SD}=0.03$ ), thus remaining nearly constant during night and day for both species. Continuous night flow was also detected at all measured azimuths in Q.i. and Q.p. and was in average $\approx 0.8 \mathrm{~cm}^{3}$ $\mathrm{cm}^{-2}$ hour ${ }^{-1}$ and $\approx 0.3 \mathrm{~cm}^{3} \mathrm{~cm}^{-2}$ hour ${ }^{-1}$ respectively, with exception of the Northern azimuth in Q.i. that presented larger $J_{p}\left(1.1 \mathrm{~cm}^{3} \mathrm{~cm}^{-2}\right.$ hour $^{-1}$ ). Based on our azimuthal night-flow observations, we advise in future studies to determine $\Delta T_{\max }$ carefully in the generally recommended TDP Northern measurements (Lu et al., 2004) to avoid underestimations of $J_{p}$.

Our azimuthal measurements showed that the Northern, Southern and the Eastern azimuths contributed to $\approx 90 \%$ of the daily sap flow in both species. This observation is comparable to the HFD patterns of Pinus sylvestris L. described (Čermák et al., 2004), in which measured sap flows at three specific azimuths $\left(360^{\circ}, 270^{\circ}\right.$ and $\left.180^{\circ}\right)$ contributed to $\approx 88 \%$ of the transported water. Significantly different azimuthal observations were made by Delzon et al. (2004) who measured $J_{p-i}$ at different depths using a modification of the TDP sensor on Pinus pinaster. They did not find any significant differences between the $J_{p-i}$ at the two measured azimuths (i.e. Northern and Eastern) and concluded that the variability in sap flow radial profiles with respect to the azimuthal directions might be neglected when estimating whole-tree sap flow under the live canopy. Future radial-azimuthal measurements are recommended but only after assuring no NTG impact.

The TDP radial and azimuthal $J_{p}$ optimization in Q.i. and Q.p. was carried out following Steppe et al. (2010). The results of that optimisation for Q.i. is presented in Figure 3.12 and for Q.p. in Figure 3.13. The RMSE of the radially and azimuthally corrected $J_{p}\left(\mathrm{TDP}_{\text {final }}\right)$ versus the $J_{p}$ $\mathrm{HFD}_{\text {ref }}$ on daily basis as presented in Figure 3.6 ranged from 0.2 to 0.9 $\mathrm{cm}^{3} \mathrm{~cm}^{-2}$ hour $^{-1}$ (with average $=0.5$ and SD $=0.2$ ) for Q.i. and from 0.4 to $1.0 \mathrm{~cm}^{3} \mathrm{~cm}^{-2}$ hour-1 (with average $=0.7$ and $\mathrm{SD}=0.3$ ) for Q.p. The close match between $J_{p}\left(\mathrm{TDP}_{\text {final }}\right)$ and $J_{p} \mathrm{HFD}_{\text {ref }}$ can also be observed on the hourly patterns between the $\mathrm{HFD}_{\text {ref }}$ and $\mathrm{TDP}_{\text {final }}$ in Figure 3.7 and 3.8 and when comparing the TDP-optimized $J_{p}$-i averages in Figures $3.12 \mathrm{k}$ and $3.13 \mathrm{k}$ with corresponding HFD measurements in Figure 3.9a and $3.9 \mathrm{~b}$.

All the individual Q.i. TDP-optimised radial $J_{p-i}$ profiles (Figure 3.12) followed typical pattern of $J_{p-i}$ decreasing with sapwood-depth and had comparable magnitudes. In all 10 measured trees, the outermost $J_{p}(0.5$ $\mathrm{cm}$ ) was in range between 5 and $7 \mathrm{~cm}^{3} \mathrm{~cm}^{-2}$ hour ${ }^{-1}$. The deeper sections of the sapwood presented significant activity until $4.5 \mathrm{~cm}$ depth, with the average of the $J_{p-i}$ maxima fluctuating between 2.0 and $4.2 \mathrm{~cm}^{3}$ $\mathrm{cm}^{-2}$ hour $^{-1}$. The average $J_{p-i}$ maxima of the deepest sections of the sapwood, i.e. between 5.5 and $7.5 \mathrm{~cm}$, were less distinct $\approx 1.6 \mathrm{~cm}^{3} \mathrm{~cm}^{-2}$ hour $^{-1}$ (Figure 3.13-l) but significant as can be observed in the gradual decrease of depth-wise $J_{p}$ at the instants of maximum $J_{p}$ at 13:15 and 15:00 (Figure 3.12-l). The non-negligible night flow detected for Q.i. can 
also be observed in the optimised measurements of the trees with DBH 30.5, 37.0 and 97.1 .

We also observed that $J_{p-i}$ for Q.i. varied with DBH tree size (Figure 3.12). For example, the daily $J_{p-i}$ maxima at $0.5 \mathrm{~cm}$ depth were larger for thinner trees $\left(6-7.9 \mathrm{~cm}^{3} \mathrm{~cm}^{-2}\right.$ hour $^{-1}$ for DBH 17 to $\left.37 \mathrm{~cm}\right)$ and smaller for thicker trees $\left(5-6.4 \mathrm{~cm}^{3} \mathrm{~cm}^{-2}\right.$ hour $^{-1}$ for DBH 50 to 97 $\mathrm{cm})$. The $J_{p-i}$ of the other HFD measuring depths $(1.5-7.5 \mathrm{~cm})$ followed the same daily "Gaussian" pattern across DBH sizes but with lower $J_{p-i}$ for each consecutive depth. However, the observed Q.i. $J_{p-i}$ variability was not distinct enough to define its clear relationship with DBH. This Q.i. relationship was also investigated in Portugal by David et al. (2007) who used radial $J_{p-i}$ profiles to estimate sapwood depth by using 6 measuring points version of the HFD designed according to (Nadezhdina et al., 1998). They found that in trees with a DBH ranging from 30 to $43 \mathrm{~cm}$, only $30 \%$ of the outer stem radius was conductive, whereas in our measurements with comparable DBH (30.5 and $37 \mathrm{~cm}$ ), we found that $84 \%$ of the outer stem radius was conductive. These differences in the sizes of conductive sapwood areas suggest that the $A_{x}$ and the radial $J_{p-i}$ profiles vary significantly among Q.i. trees depending on the environmental conditions of growth, i.e. depending on climate (it is drier in Portugal than in Sardón), soils (sandy Cambisol in Portugal Vs. eutric Cambisol in Sardón), depth to water table (average during the dry season $\approx 13$ meters in Portugal and $\approx 5$ meters in Sardón) and likely some other environmental factors.

In the case of Q.p. (Figure 3.13) the TDP-optimisation of the 10 measured trees also resulted in the $J_{p-i}$ radial profiles decreasing with sapwood depth. However in contrast to Q.i., the $J_{p-i}$ of the outermost section of the sapwood, indicated substantially larger $J_{p}$ at $0.5 \mathrm{~cm}$ sapwood depth, expressed by significantly larger maxima ranging from 8 $\mathrm{cm}^{3} \mathrm{~cm}^{-2}$ hour ${ }^{-1}$ to $12 \mathrm{~cm}^{3} \mathrm{~cm}^{-2}$ hour ${ }^{-1}$ The deeper sections of the sapwood presented significant activity until $3.5 \mathrm{~cm}$ depth, with the $J_{p-i}$ maxima ranging between 3.0 and $5.0 \mathrm{~cm}^{3} \mathrm{~cm}^{-2}$ hour $^{-1}$. The deepest sections of the sapwood, i.e. between 4.5 and $7.5 \mathrm{~cm}$, had less significant $J_{p-i} \approx 1.8 \mathrm{~cm}^{3} \mathrm{~cm}^{-2}$ hour $^{-1}$ (Figure 3.13). In general the $J_{p}$ radial profile of Q.p. indicated less distinct activity in the deep sections of a the sapwood which can be observed through steep decrease of the depth-wise $J_{p}$ at the instants of maximum activity at 14:15 and 15:00 (Figure 3.13-l). The non-negligible night flow detected for Q.p can also be observed for all the optimised measurements.

We did not find any clear relationship between the $J_{p-i}$ of $Q . p$. and the DBH sizes of the investigated trees (Figure 3.13). The Q.p. species was earlier studied by Hernández-Santana et al. (2008a) in a humid Rinconada catchment in Spain with yearly rainfall of $939 \mathrm{~mm}$. They measured the $J_{p}$ using 1 and $2 \mathrm{~cm}$ long TDP probes to have an approximation of the $J_{p}$ radial variability in trees with a mean $\mathrm{DBH}$ of $11.7 \mathrm{~cm}$ and $\mathrm{SD}=3.9 \mathrm{~cm}$. They found a proportion of 65:35 between the maximum $J_{p}$ of the first and the second centimetre of the sapwood. Using HFD we found this ratio to be very close to ours (64:36) despite much drier conditions during 
our measurements. We also found that the ratio for $1 \mathrm{~cm}$ thick sapwood layers calculated from the average of the $J_{p-i}$ maxima of the 10 trees for each sapwood depth from outside to inside were: 44:25:10:7:5:4:3:2. These calculations confirm that by using standard TDP sensors without correction, the measured $J_{p}$ can be highly overestimated in Q.p.

Accounting for radial and azimuthal variability is one of the most important factors to consider for proper water accounting using the TDP method. Sap flow $\left(Q_{s}\right)$ is a product of $J_{p}$ and sapwood depth $\left(l_{s}\right)$ therefore if the sapwood depth is larger than the length of the probe (in TDP $2 \mathrm{~cm}$ ), the uncertainty in the $Q_{s}$ estimate is introduced. This uncertainty is because $J_{p}$ is typically non-uniform along the radial axis of sapwood depth as confirmed by our HFD measurements. This study, but also other sap flow studies (Čermák et al., 1998; Ford et al., 2004; Nadezhdina et al., 2007; Saveyn et al., 2008) confirmed large azimuthal $J_{p}$ variability which also significantly influences sap flow estimates. Therefore, azimuthal variability of $J_{p}$ needs larger scientific attention. By applying the proposed optimization method, the TDP method in combination with reliable reference method, can contribute to sap flow estimates accounting for radial and azimuthal $J_{p}$ sapwood variability of trees with various DBH, also those with $l_{s}>2 \mathrm{~cm}$.

\subsection{Opportunities and final conclusions}

The dry season measurements carried out in the sparse woodland in Spain, confirmed significant impact of NTG on TDP $J_{p}$ measurements. The applied CHD method as a first step of the TDP optimization, removed that impact confirming to be appropriate and indispensable in the processing of TDP data acquired in the environmental conditions similar to the presented in this study. The application of CHD method on two thermally different tree species characterized by different NTG, showed that $J_{p}$ of Q.p. trees characterized by relatively low NTG in order of +/- 0.2 deg, could be comparably affected by NTG as the $J_{p}$ of $Q . i$. trees characterized by large NTG in order of +/- 1.0 deg. The second and the third steps of the proposed TDP optimization, require reference, calibration method. In this study the HFD method was used as such reference method although other methods providing reliable input for the second and third steps could also be used provided they are: (a) accurate and portable, e.g. optimal in this regard would be portable NMR method, unfortunately it has no commercial field implementation yet; (b) sensitive enough to low flows as to discriminate zero flow and the change of flow direction and (c) are able to provide radial-azimuthal sap flow measurements.

Our results have highlighted the importance of applying the proposed corrections to standard TDP measurements particularly when carried out under dry-conditions over the sparse vegetation. For example, $J_{p}$ acquired according to standard TDP procedures without optimisation $\left(\mathrm{TDP}_{s t d}\right)$ as presented in Figure 3.4 and 3.5, was on average $>100 \%$ 
higher than the optimized $J_{p}\left(\mathrm{TDP}_{\text {final }}\right)$ for $Q . i$. and $70 \%$ higher than $\mathrm{TDP}_{\text {final }}$ for Q.p. If the TDP $\mathrm{T}_{\text {std }}$ was used to calculate water-budgets for the Sardón catchment, the derived transpiration would be almost twice overestimated. For that reason, we advise caution in the use of TDP sapflow data without appropriate correction. The proposed in this study, step-wise optimisation method, offers a solution that combines the TDP efficiency and cost effectiveness with the accuracy and versatility of the HFD, thus greatly improving the capability of sap flow measurements. This is particularly important for large data collection studies such as catchment tree transpiration mapping that require accurate investigation of significant numbers of trees of various species and sizes in a costeffective manner. The step-wise optimisation method was developed to support such transpiration mapping of the Sardón catchment carried out by remote sensing upscaling of sap flow measurements 4 .

Besides the methodological issues related to the proposed TDP optimisation method, this study presents also valuable dry season sap flow measurements of Q.i. and Q.p. highlighting individualities of these species while exposed to water stress. Particularly valuable in that respect is the novel information on radial and azimuthal variability of sap flux density $\left(J_{p}\right)$ of Q.i. and Q.p. highlighting traits not described before, namely: (1) continuous and significant night time sap flow in all investigated Q.p. trees and exceptionally also in Q.i.; (2) exponentially decaying $J_{p}$ with sapwood depth with significantly larger $J_{p}$ in the outermost $1 \mathrm{~cm}$ of Q.p. sapwood than in in the outermost $1 \mathrm{~cm}$ of Q.i.; (3) high azimuthal $J_{p}$ variability for Q.i. and Q.p. These findings can contribute to a better understanding of the dynamics governing the plant-soil-water interactions in water limited environments this way also contributing to the improvement of landscape and water resources management. 

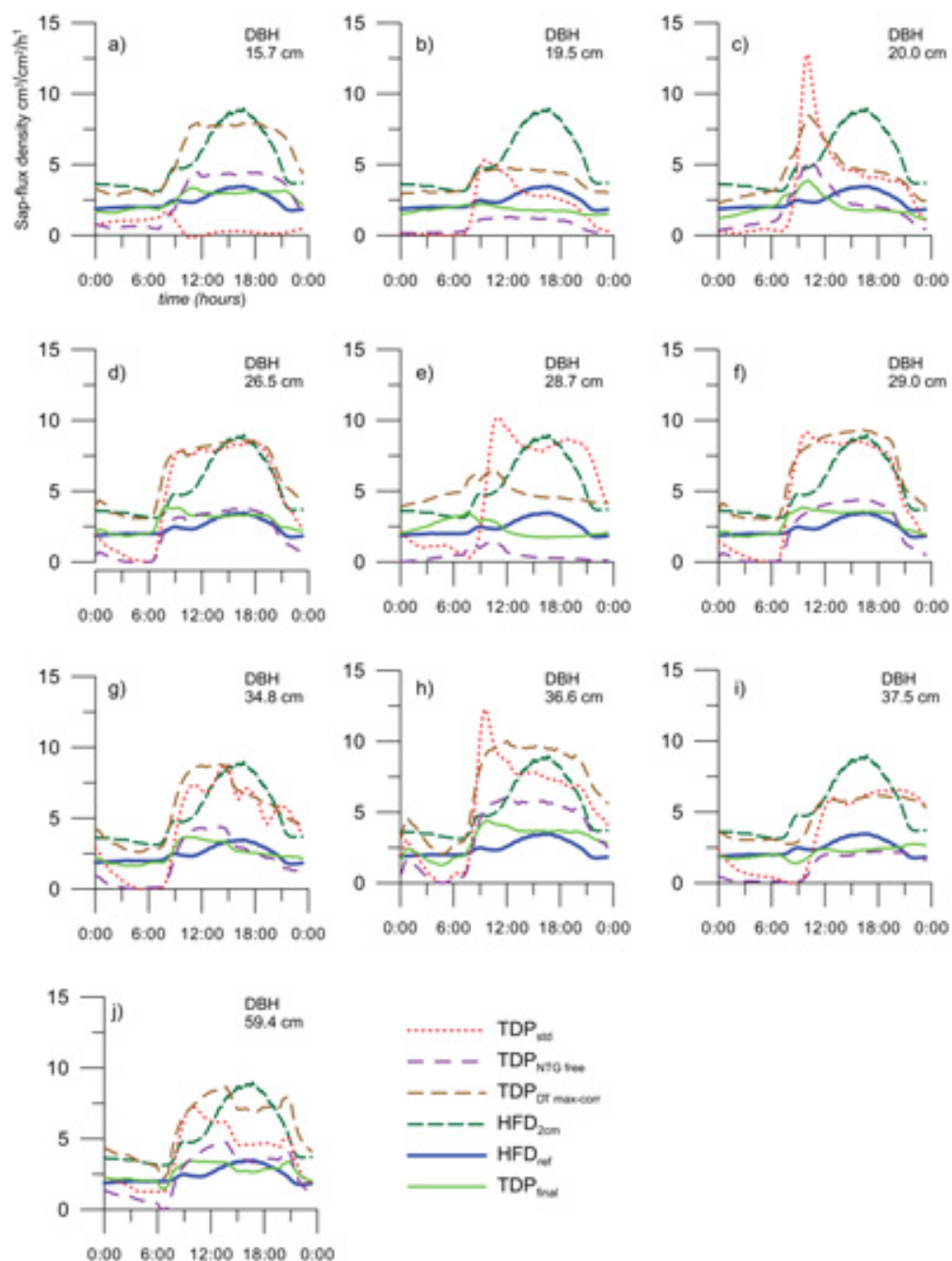

Figure 3.8: Step-wise optimisation of TDP $J_{p}$ measurements using HFD as reference, for 10 selected Q.p. trees (a-j): $\mathrm{TDP}_{s t d^{-}}$uncorrected measurement (dotted red line); TDP $_{N T G-\text { free }}$ NTG-corrected $J_{p}$ (dashed violet line); $\mathrm{TDP}_{\Delta \mathrm{Tmax} \text {-corr }}$-adjusted for night flow (dashed dark-yellow line);

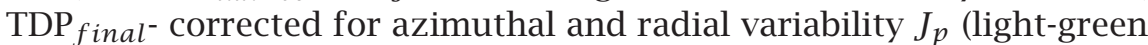
line); $H F D_{2 \mathrm{~cm}}$ - reference for the $2 \mathrm{~cm}$ long TDP sensors (dashed darkgreen line); and $\mathrm{HFD}_{r e f}$ - (bold blue line) radial-azimuthal $J_{p}$ reference (the time axis follows GMT+1 Spanish summer time). 

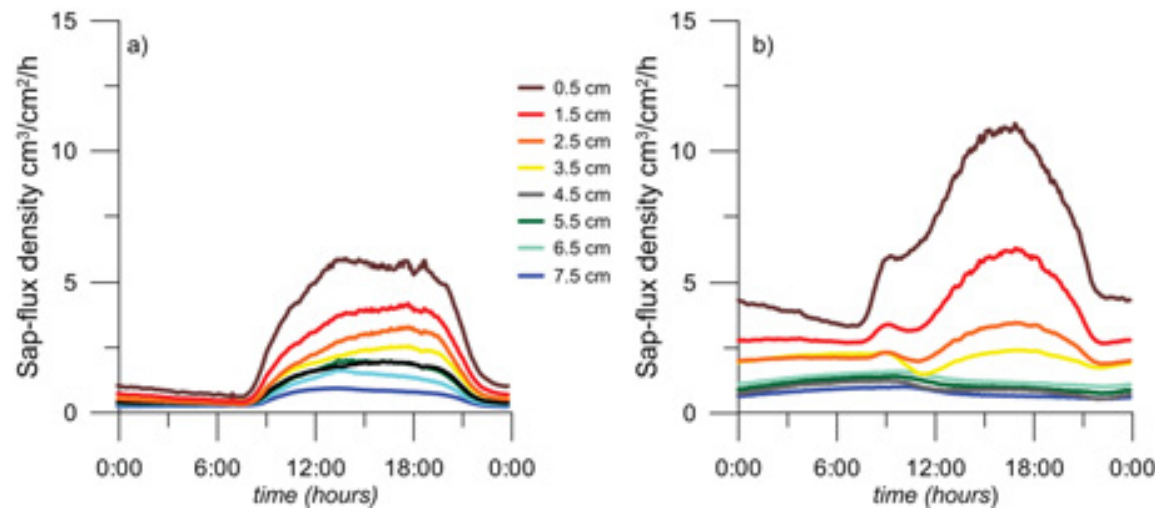

Figure 3.9: Average $J_{p-i}$ radial variability of (a) 10 Q.i. trees and (b) 10 Q.p. trees of different DBH sizes measured at the Northern azimuth with the HFD sensor (the time axis follows GMT+1 Spanish summer time).
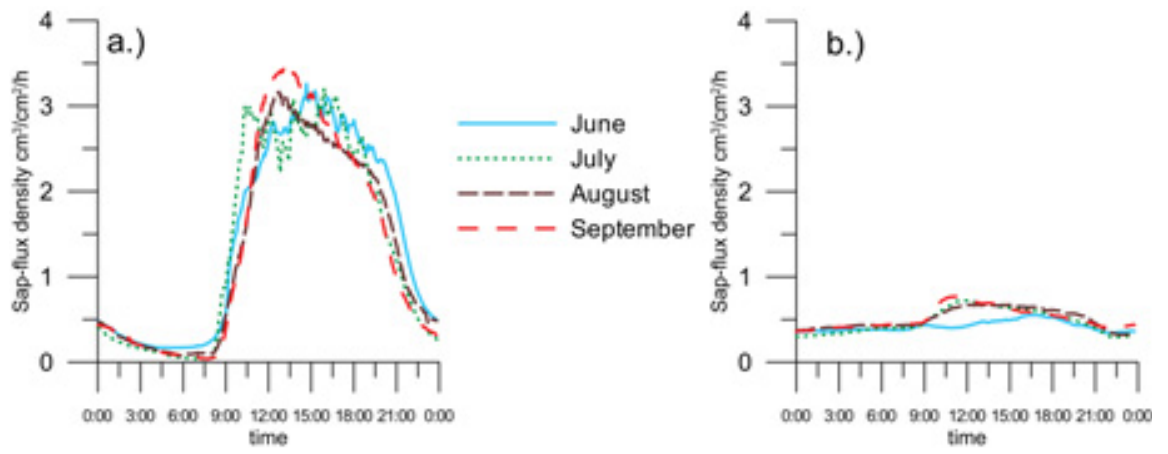

Figure 3.10: Dry season, monthly variability of HFD $J_{p-i}$ measurements (average of 8 measured depths) in: (a) Q.i. and (b) Q.p. for the most representative $\mathrm{DBH}$ size categories, i.e. $\mathrm{DBH}=20-50 \mathrm{~cm}$ (the time axis follows GMT +1 Spanish summer time). 

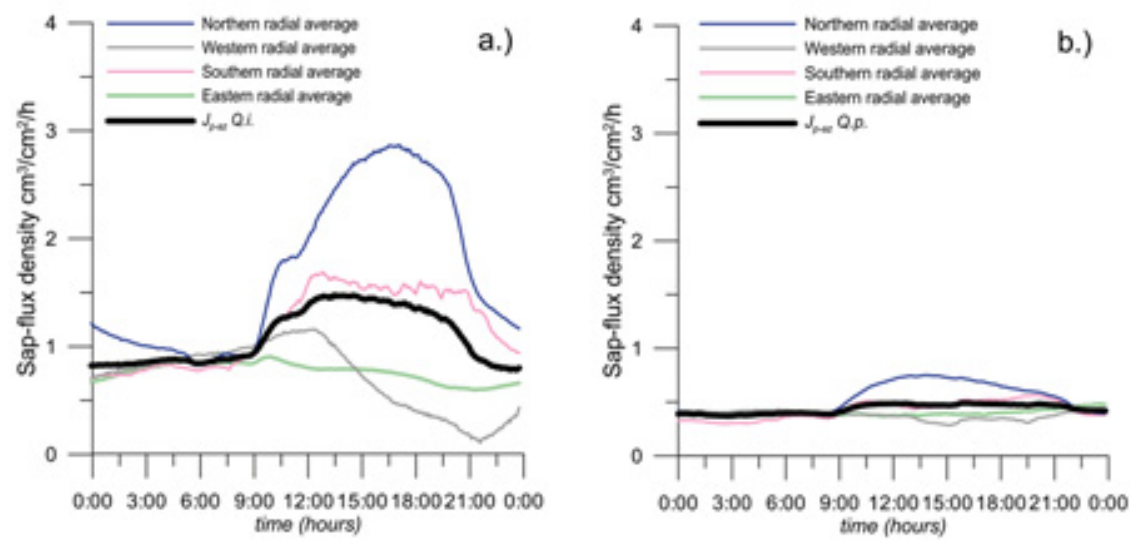

Figure 3.11: HFD azimuthal variability (Northern, Southern, Western and Eastern azimuths) of sap flux density $\left(J_{p-a z}\right)$ as average of 8 measured depths in: (a) 2 Q.i. trees and (b) 2 Q.p. trees, calculated by averaging the azimuthal measurements of four trees per species for the most representative DBH categories ranging from 20 to $50 \mathrm{~cm}$ (the time axis follows GMT+1 Spanish summer). 

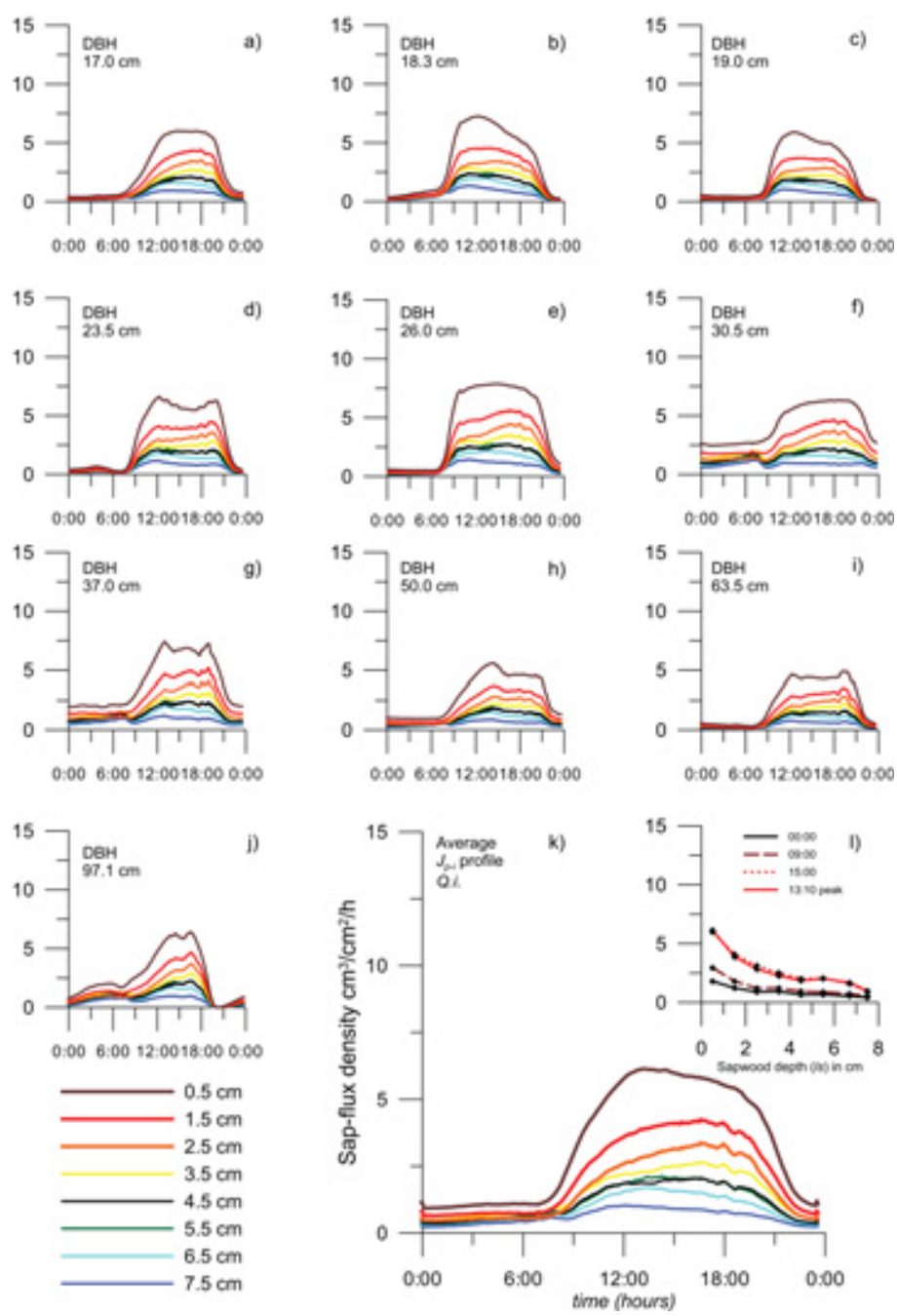

Figure 3.12: Optimised $J_{p-i}$ radial variability ( $\mathrm{TDP}_{\text {final }}$ ) of 10 Q.i. trees of different DBH sizes measured at the Northern azimuth $(a-j)$. The Figure $\mathrm{k}$ shows daily $J_{p-i}$ averages over the 10 trees and the Figure 1 radial depth-wise profiles at 4 different instants (the time axis follows GMT+1 Spanish summer time). 

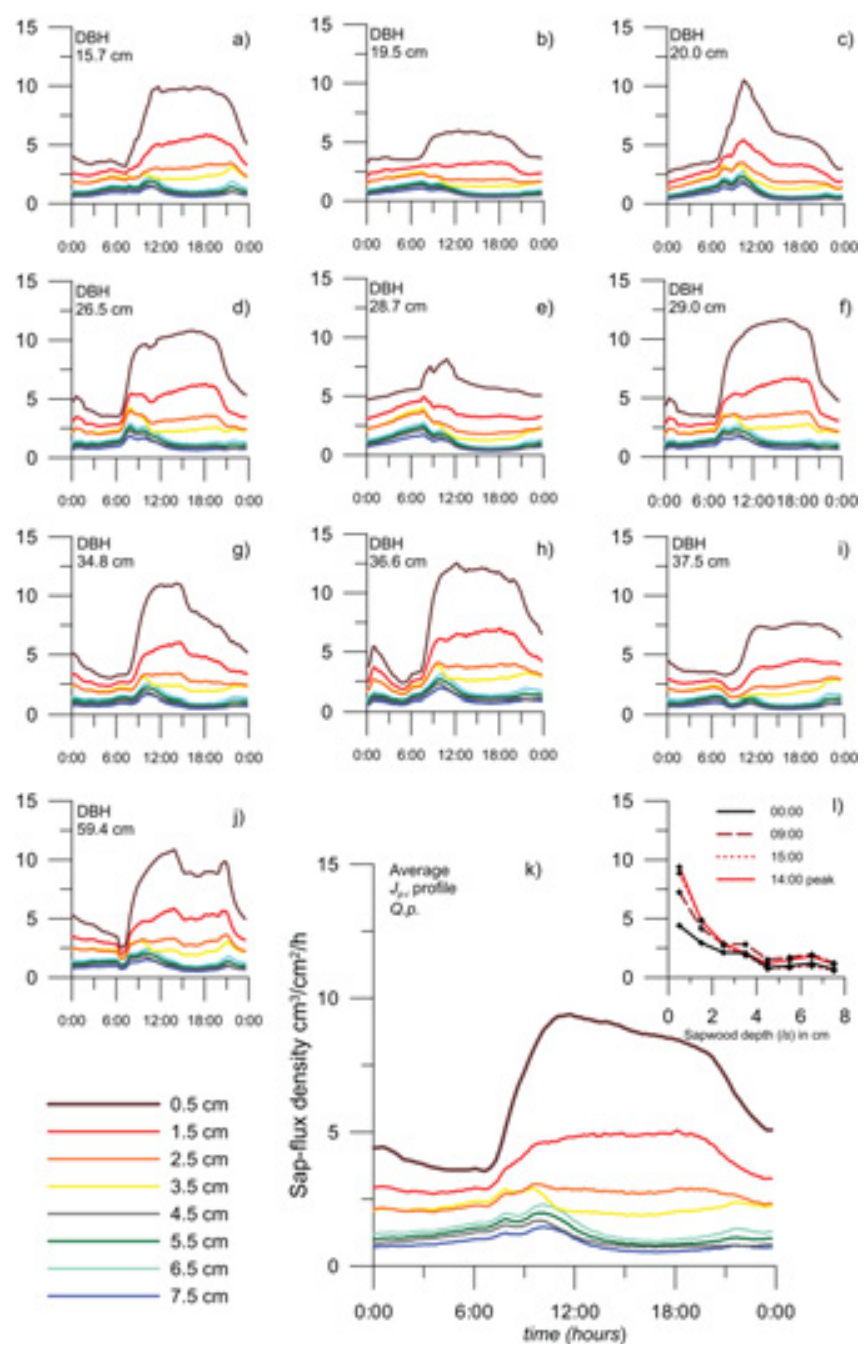

Figure 3.13: Optimised $J_{p-i}$ radial variability ( $\mathrm{TDP}_{\text {final }}$ ) of 10 Q.p. trees of different DBH sizes measured at the Northern azimuth $(a-j)$. The Figure k shows daily $J_{p-i}$ averages over the 10 trees and the Figure $\mathrm{l}$ radial depth-wise profiles at 4 different instants (the time axis follows GMT+1 Spanish summer time). 



\section{Dry-season tree transpiration in the Sardón catchment, scaled up from satellite imagery at different spatial scales}

Figure 4.1: QuickBird multispectral image highlighting in red the tree canopies of the open-woodland (false-colour near-infrared compos-

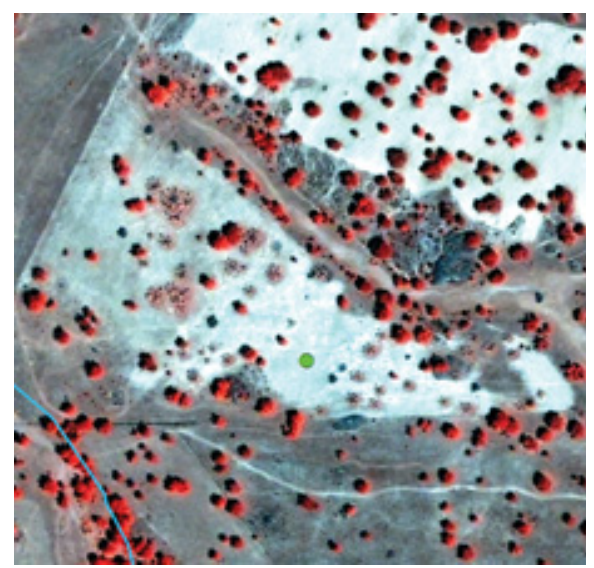
ite band combination: 4-2-1) at the Sardón catchment in Salamanca, Spain. The location of the Eddycovariance tower is highlighted in green and the blue lines represent the location of the main water stream.

This Chapter is based on: Mapping dry-season transpiration of an oak wood-land in Salamanca, Spain. Reyes-Acosta, J.L. and M.W. Lubczynski. (2012). Published in Agricultural and Forest Meteorology. 


\begin{abstract}
Tree transpiration is an important plant-physiological process that influences the water cycle, thereby influencing ecosystems and even the quantity of available water resources. However, direct tree-transpiration measurements, particularly at large spatial scales, are still rare, due to the complexities associated with natural ecosystems. This study describes a scaling-up method for quantifying dry-season tree transpiration $\left(T_{c}\right)$ of the Sardón catchment, located in Central-Western Spain, $50 \mathrm{~km}$ west of Salamanca. The method is applied to an oak woodland in that catchment which is dominated by two tree species: evergreen Quercus ilex and deciduous Quercus pyrenaica (Q.i. and Q.p., respectively). The method comprises five complementary steps: (a) sap-flux density $\left(J_{p}\right)$ measurement; (b) definition of biometric-upscaling functions; (c) spatial scaling-up of tree transpiration using high-resolution remote-sensing-derived object attributes (50 - $60 \mathrm{~cm}$ per pixel); (d) modelling of dry-season temporal sap-flow variability; and (e) an assessment of the robustness/uncertainty of the method. The proposed method was applied to assess dry-season tree transpiration, however if there is appropriate sap flow data available, it can also be applied during any season of the year. The application of the proposed method to map tree transpiration in the Sardón catchment resulted in a mean $J_{p}=58.4 \mathrm{~cm}^{3} \mathrm{~cm}^{-2}$ day $^{-1}$ for $Q . p$., characterised by tree density of 19 trees ha ${ }^{-1}$, and a mean $J_{p}=37.1 \mathrm{~cm}^{3} \mathrm{~cm}^{-2}$ day $^{-1}$ for Q.i., characterised by tree density of 4 trees $\mathrm{ha}^{-1}$. The $J_{p}$ data varied as dependent on the weather conditions and not on the change of soil moisture. The mean normalised tree transpiration $\left(T_{t}\right)$ estimated per species, was also higher for Q.p. $\left(1.19 \mathrm{~mm} \mathrm{day}^{-1} \pm 8 \%\right)$ than for Q.i. (0.83 $\left.\mathrm{mm} \mathrm{day}^{-1} \pm 25 \%\right)$ trees. The remote-sensing scaling up of tree transpiration for the Sardón catchment resulted in a low mean dry-season $T_{c}=0.045 \mathrm{~mm} \mathrm{day}^{-1}( \pm 1 \%)$ typical for water limited environments with sparse tree coverage (average $\approx 7 \%$ ). In that $T_{C}$ Q.p. represented $79 \%$, whereas Q.i. $21 \%$. The dry-season $T_{\mathcal{C}}$ varied spatially at 1 ha resolution but barely temporally throughout the dry season. The obtained $T_{c}$ maps can be readily used as input for hydrological models in water resources and land management decision-making.
\end{abstract}

\title{
4.1 Introduction
}

Assessment of tree transpiration between and within different biophysical scales in the soil - plant - atmosphere continuum is an appropriate approach for understanding the role vegetation plays in hydrology (Lubczynski, 2000; David et al., 2004; Hernández-Santana et al., 2009; Lubczynski, 2009). Accurate quantification of transpiration dynamics is crucial in fields such as water resources assessment (Lubczynski, 2000, 2009), forest management (Brown et al., 2005), agricultural practices (Lu et al., 2002), and for acquiring a better understanding of climate change (Martínez-Vilalta et al., 2002), to mention just a few. Diverse approaches 
to measure transpiration in-situ (Čermák et al., 1982; David et al., 2007; Devitt et al., 1993; Hernández-Santana et al., 2008a) and/or indirectly (Calder, 1978; Caspari et al., 1993; Moore et al., 2008; Verbeeck et al., 2007) have been reported in the literature. The challenge still remains, however, to represent transpiration at higher, for example, catchment scales.

Typically, the tree transpiration studies have attempted to quantify transpiration of stands of pristine, semi-natural or cultivated forests (Čermák and Kučera, 1990; Hatton and Wu, 1995; Granier, 1996; Vertessy et al., 1997; Köstner et al., 1998; Wullschleger et al., 1998; Lundblad and Lindroth, 2002; Ford et al., 2007; Nagler et al., 2007), the vegetation cover of which was closed and dominated by tree canopies. In such stands, the classical procedure is to establish species-specific, mathematical functions, relating sap flow with the stem size based on some few measurements of the selected trees. Such functions allow the inference of transpiration for the trees not measured (Čermák et al., 2004). Such approach can be technically complicated and thus of limited use for large scales studies of sparse forests in water-limited environments (WLE), such as Mediterranean dehesas in Spain (Lubczynski and Gurwin, 2005; Reyes-Acosta and Lubczynski, 2014) or the savannahs of the Kalahari (Kimani et al., 2007; Chavarro-Rincón, 2009). Such forests are characterised by highly variable tree sizes and sparsely located trees (distance between trees can be $>10$ metres), which makes direct data collection and the establishment of a robust mathematical relations between biometric characteristics and sap flow difficult, and the acquisition of all the stem characteristics necessary for scaling up large areas, practically impossible. Therefore a reliable alternative is to use remote sensing for scaling tree sap flow measurements.

The remote sensing based scaling up of tree sap flow measurements has been demonstrated already by Čermák and Kučera (1990) who argued that such scaling up procedure is a good alternative for estimating tree transpiration at high spatial scales. They described an approach focused on determining a mathematical relation between sap flow and reflectance of the foliage of canopies in the near-infrared region of the spectrum (band 7 from multispectral images acquired with a double lens reflex camera).

More recently, Boegh et al. (1999), Nagler et al. (2007), Murray et al. (2009) and Cristóbal et al. (2011) have further explored the possibilities of using remotely-sensed images to quantify evapotranspiration at large scales $(E T)$ by using vegetation indices, for example the Normalized Difference Vegetation index (NDVI) and the Enhanced Vegetation Index (EVI). Boegh et al. (1999) calculated these indices using Landsat-TM and Spot images (20 m spatial resolution). Nagler et al. (2007) and Murray et al. (2009) used images from the Moderate Resolution Imaging Spectrometer (MODIS) (250 m spatial resolution), whereas Cristóbal et al. (2011) used a combination of both. Boegh et al. (1999) proposed that the status of such indices can be mathematically related to transpiration $(T)$, while Nagler et al. (2007); Murray et al. (2009); Cristóbal et al. (2011) have argued that 
they can be related to $E T$. Each of these five studies, however, is based on the electromagnetic properties of the vegetation and not on the attributes of the objects in the images: size, shape, colour, and compactness, for example. Furthermore, the spatial resolution of these Landsat-TM, Spot and MODIS images do not allow the definition of individual canopies. In addition, the approach using MODIS cannot discriminate yet between evaporation and transpiration; and the associated errors are 20\% to 30\% (Nagler et al., 2007; Murray et al., 2009; Cristóbal et al., 2011). All these factors reduce the applicability of vegetation indexes derived from electromagnetic properties to quantify $T$ in sparse vegetation areas of WLEs.

As the spatial resolution of satellite-based products has significantly increased during the last decade, high-resolution remotely-sensed images are now readily available for detecting object attributes of single canopies, facilitating the mapping of transpiration in large catchment scales. The remote-sensing method of scaling-up sap flow measurements for transpiration mapping is especially suitable for quantifying transpiration of sparse vegetation composed of a small amount of tree species (Lubczynski, 2009), so that the probability of misclassification of tree species is minimized (Kimani et al., 2007; Lubczynski, 2009; ChavarroRincón, 2009). The first attempt to use object attributes from high-spatial resolution remotely-sensed images to scale up sap flow measurements in the Kalahari Desert was reported by Chavarro-Rincón (2009). They demonstrated that the main advantage of scaling-up single-tree sap flow measurements to large scale estimates using high-resolution images, was the higher accuracy and resolution of the tree transpiration estimates (tree level). In this study, this approach has been further developed and evaluated by: (i) correlating canopy areas defined from satellite imageries with sapwood areas and not with sap flow measurements following Lubczynski (2009); (ii) including radial and azimuthal sap flux density measurements $\left(J_{p-i}\right)$ in the scaling-up process after Reyes-Acosta and Lubczynski (2014), and by (iii) evaluating its uncertainty.

The aims of this study were: (a) to scale-up tree transpiration estimates in $\mathrm{mm} \mathrm{day}^{-1}$, to a grid of 1 ha resolution, based on in-situ sap flow measurements of individual evergreen (Quercus ilex subsp. ballota) and deciduous (Quercus pyrenaica Willd) oak trees (Q.i. and Q.p., respectively) to represent spatial variability of tree transpiration at the stand and catchment scales; (b) to simulate the temporal variability of sap flow $\left(Q_{s}\right)$ during the dry-season and describe its implications for spatial transpiration estimates; and, finally, (c) to evaluate the robustness and uncertainties of scaling up transpiration by using attributes derived from identified objects in remotely-sensed images.

\subsection{Materials and Methods}

The tree transpiration of the Sardón catchment was quantified spatially by combining two complementary steps: (a) sap flow measurement, and 
(b) the spatial scaling up of the sapwood area of individual trees using object attributes identified in high-resolution remotely-sensed images. These steps are described in the following sections.

This study was focused on the dry season because sap flow data collected during this period is typically the most relevant for (and demanded by) hydrologists (Lubczynski, 2009). Therefore, the dry season conditions were analysed and standardised as follows: absence of rainfall, continuous periods of clear-sky days, daily peaks of solar radiation $>700 \mathrm{~W} \mathrm{~m}^{-2}$, and maximum daily vapour pressure deficits $>1.5 \mathrm{kPa}$. The sap flow measurements and tree transpiration maps obtained in this study are assumed to be valid under such standardised conditions.

\subsubsection{Study Area}

This study was developed in the Sardón catchment $\approx 80 \mathrm{~km}^{2}$ (Figure 4.2), which is characterized by low population density and, with that, equally low levels of human impact (Lubczynski and Gurwin, 2005). The climate of the Sardón study area is semi-arid, with a mean rainfall of $\approx 500 \mathrm{~mm} \mathrm{yr}^{-1}$ (Lubczynski and Gurwin, 2005). The warmest and the driest months are July and August, with an average temperature of $22^{\circ} \mathrm{C}$, potential evapotranspiration (PET) of $5 \mathrm{~mm} \mathrm{day}^{-1}$ calculated from the Penman-Monteith equation (Allen et al., 1998) and rainfall $<3$ mm month ${ }^{-1}$ (Lubczynski and Gurwin, 2005). The bare soil evaporation during dry season is $\approx 0.55 \mathrm{~mm}$ day $^{-1}$, as estimated by a liquid and vapour water flow model calibrated against soil moisture measurements (Balugani et al., 2011).

The Sardón catchment is composed of massive, fractured and weathered granites with inclusions of schists and gneisses. The superficial, weathered, unconsolidated material is only a few metres thick and is widely intercalated by solid granite outcrops. The water-table is hydraulically connected to intermittent streams in valleys and at topographic elevations rests some few metres below the ground surface. The study area is a woodland with scattered patches of woody-shrub vegetation (Cytisus scoparius, also known as Scotch Broom) and a semi-natural open forest containing two dominant tree species: the evergreen oak Quercus ilex subsp. ballota Desf. (Samp.) (Q.i.) and the broad-leafed deciduous oak Quercus pyrenaica Wild (Q.p.). Q.i. trees have small sclerified leaves and are well adapted to drought stress (Manes et al., 2006), whereas Q.p. trees have broad lobulated leaves and grow better in sub-humid/humid environments, although they also tolerate dry conditions (Hernández-Santana et al., 2008c). The understory vegetation in the Sardón catchment is mainly composed of summer-annual grasses that wilt during the dry season, therefore their dry season water uptake was considered negligible in this study.

The climatic conditions of the Sardón catchment during summer are characteristic of a WLE. sap flow measurement under such WLE conditions is challenging due to the sensitivity of the measurement techniques 
to a number of potential inaccuracies, summarised in Subsection 4.2.3 below.

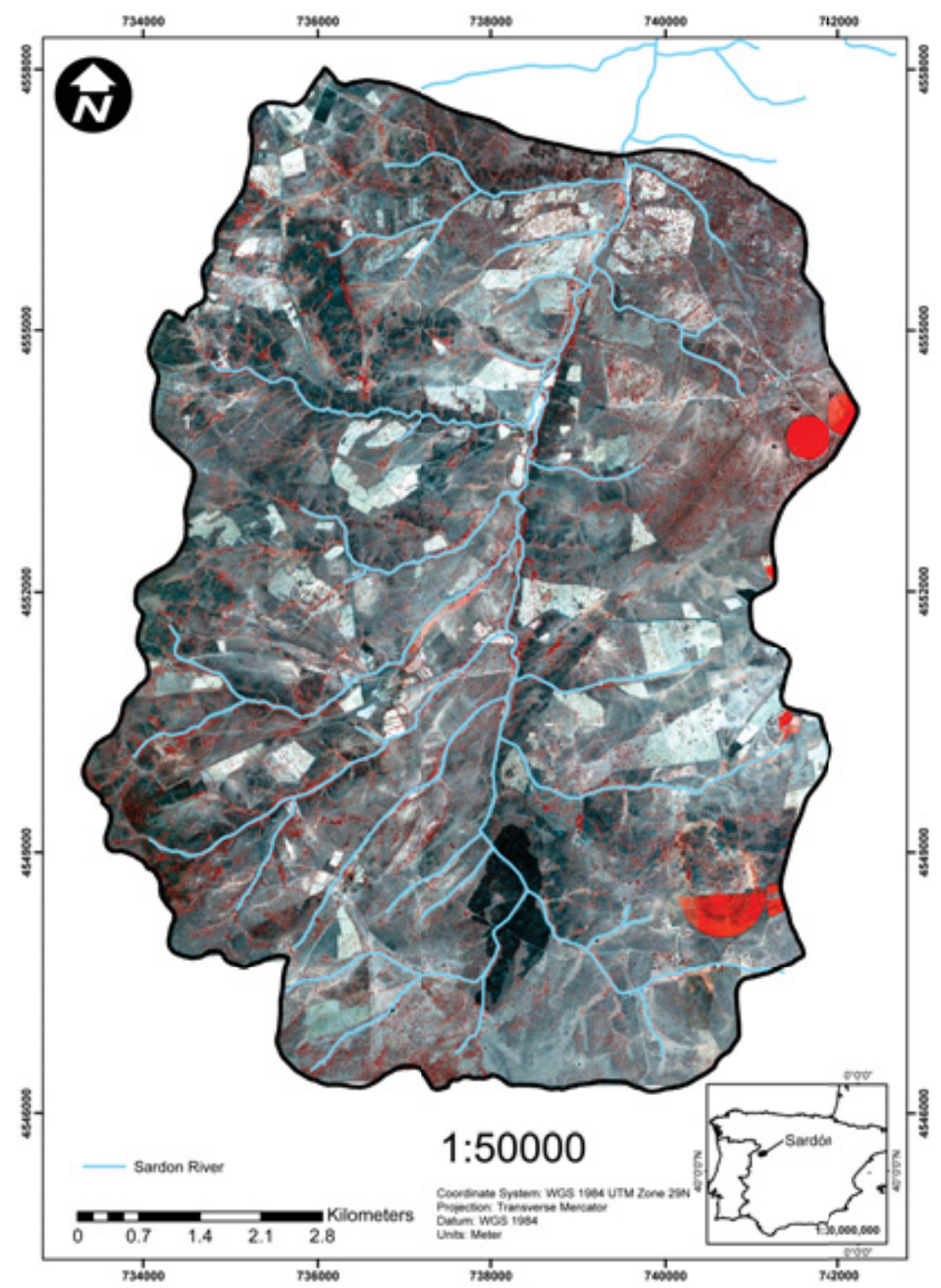

Figure 4.2: "QuickBird" false colour image (Near-infrared composite band combination: 4-2-1) from the Sardón catchment highlighting the infrared portion of the vegetation (vegetation displayed in red) in August $9^{\text {th }}$ of 2009 (The red semi-circles are grasses cultivated for pasture).

\subsubsection{Measurement of biometric characteristics}

The biometric characteristics of oak trees in the Sardón area were surveyed for use in the scaling-up process. A full description of the survey 
design can be found in Reyes-Acosta and Lubczynski (2014). The survey focused on obtaining the biometric characteristics necessary to define the attributes of the tree population, for example: diameter at breast height $(\mathrm{DBH})$, cross-sectional area of the tree stem, $\left(A_{s}\right)$, ground projected canopy area $\left(A_{c}\right)$ and sapwood area $\left(A_{x}\right)$. These measurements were obtained from 90 Q.i. and 84 Q.p. trees (see Figure 4.2 for their location) and further processed to statistically define the tree-size categories (i.e. structure of the population). In this study the $A_{x}$ was selected as an appropriate parameter to define the population structure for scaling up transpiration because it has been related to the state of growth of the trees (Gea-Izquierdo et al., 2009) and has a low temporal variability. The $A_{x}$ scaled parameter was calculated by determining the depth of the sapwood in the stems using two techniques, namely: visual inspection of dyed wood-cores and by measuring depth-wise sap flux densities using the heat field deformation (HFD) method. The corresponding $A_{c}$ scalar was defined by estimating the projected area of the canopy with a minimum of two transects across the ground demarcated by $90^{\circ}$ angles with a clinometer. For further details on the $A_{x}$ and $A_{c}$ measuring field-campaign see Chapter 3.

\subsubsection{Sap flow measurements}

Sap flow measurement $\left(Q_{s}\right)$ is a widely used method for quantifying whole-plant water use (Lu et al., 2004) and can express transpiration fluxes, provided stem storage can be neglected. $Q_{s}$ measurement consists of separate measurements of sap flux density $\left(J_{p}\right)$ and sapwood (xylem) area $A_{x}$ (Čermák et al., 2004; Granier, 1985; Lu et al., 2004; Lubczynski, 2009), following Equation 4.1:

$$
Q_{s}=J_{p} \times A_{x}
$$

where $J_{p}$ is typically expressed in $\mathrm{cm}^{3} \mathrm{~cm}^{-2}$ hour ${ }^{-1}$ and xylem area $\left(A_{x}\right)$ in $\mathrm{cm}^{2}$. In this study, $Q_{s}$ was measured in Q.i. and Q.p. trees during monitoring campaigns conducted in the summer of 2009 and 2010 using thermal dissipation probes (TDP) and heat field dissipation sensors (HFD), as presented in Reyes-Acosta and Lubczynski (2014). Depending on the DBH of each tree, one to three TDPs were used.

The TDP method has been widely used in many studies (Chu et al., 2009; Granier, 1987; Köstner et al., 1998; Lu et al., 2004; Paço et al., 2009; Regalado et al., 2009) and features an empirical but widely-tested calibration to relate measured temperature differences between two thermocouples (one heated and the other not) inserted in the sapwood $\left(\Delta T\right.$ in ${ }^{\circ} \mathrm{C}$ ) at $10 \mathrm{~cm}$ distance, with sap flux density $J_{p}$ (in $\mathrm{cm}^{3} \mathrm{~cm}^{-2}$ hour $^{-1}$ ) (Granier, 1985). The TDP method has a good validation record for oak species (Granier et al., 1994). However, considering that the $2 \mathrm{~cm}$ sensing length of the standard TDP probes was not sufficient to cover the entire depth of the investigated oaks (sapwood depth $>2 \mathrm{~cm}$ ), additional 
radial measurements were necessary to properly quantify sap flow (check Reyes-Acosta and Lubczynski (2014) for the reported sapwood depths larger than $2 \mathrm{~cm}$ ).

Such measurements were made with HFD sensors (ICT International, Armidale, NSW, Australia). The HFD sensors have 8 measuring points each spaced $1 \mathrm{~cm}$ apart, starting at $0.5 \mathrm{~cm}$ through $7.5 \mathrm{~cm}$ sensing depth. The HFD method determines $J_{p}$ based on the spatial deformation of a heat field around a linear heater (placed tangentially in the sapwood of the tree being studied), represented by the ratio of the measured temperature differences symmetrically $\left(\delta T_{\text {sym }}\right)$ and asymmetrically $\left(\delta T_{\text {asym }}\right)$ (Nadezhdina et al., 1998, 2002, 2012). To determine the radial and azimuthal variations in the sampled trees, two HFD sensors were used at the same time for two days at opposite azimuths, and then rotated to measure the other opposite azimuths for additional two days. Both species, Q.i. and Q.p., presented significant radial variations deeper than $2 \mathrm{~cm}$ and also at different azimuths. More information on the fieldwork setup and on the assessment of the radial and azimuthal variabilities can be found in Reyes-Acosta and Lubczynski (2014).

The objective behind combining the TDP and the HFD methods was to optimise sap flow measurement by taking advantage of the robustness and cost-effectiveness of TDP sensors, and the enhanced xylem-depths resolution of HFD sensors, which unfortunately are of limited use because of their high cost. The applied optimisation allowed handling the three types of common TDP inaccuracies, related to: (1) biases from the influence of natural thermal gradients (NTG) (Do and Rocheteau, 2002a,b; Lu et al., 2004; Lubczynski et al., 2012; Reyes-Acosta et al., 2012); (2) radial and azimuthal variability of $J_{p}$ (Nadezhdina et al., 2002; Poyatos et al., 2007); and (3) night-flow in trees and related uncertainty in the assumption of a $\Delta T_{\max }$ value (Reyes-Acosta and Lubczynski, 2014).

The trees assigned for sap flow measurements were selected by: (1) determining their size category; (2) making sure they had symmetrical stems; and (3) verifying that they did not present any apparent sign of decay (hollow trunks) and/or illness (e.g. presence of infected black tissue in core samples). To properly account for tree-size variability in the population, but with minimum amount of measurements, the TDP and HFD sap flow measurements followed a stratified sampling scheme. The stratified sampling comprised distributing a 10-trees sample between the tree-size categories established from the biometric measurements of $A_{x}$ and measuring the corresponding $J_{p}$ for each of those categories. Next the $J_{p}$ and the sap flux density for the outermost $2 \mathrm{~cm}$ of the xylem $\left(J_{p 2 \mathrm{~cm}}\right)$ were plotted against corresponding $A_{x}$ as in Figure 4.3. In this figure, based on $J_{p 2 c m}$, an arbitrary categorization of the sap flux density as dependent on $A_{x}$ has been made. The assigned $J_{p 2 c m}$ categories were as follow: (1) "Small", for Q.i. trees trees with $A_{x}<300 \mathrm{~cm}^{2}$ and for Q.p. with $A_{x}<200 \mathrm{~cm}^{2}$; (2) "Medium" for Q.i. trees with $300 \mathrm{~cm}^{2} \leq A_{x}<400$ $\mathrm{cm}^{2}$ and for Q.p. with $200 \mathrm{~cm}^{2} \leq A_{x}<400 \mathrm{~cm}^{2}$; and (3) "Large" for both tree species with $A_{x} \geq 400 \mathrm{~cm}^{2}$. 
Table 4.1: Average $J_{p i}$ profile for the first two centimetres $(0.5$ to $1.5 \mathrm{~cm})$ in the tree-size categories for Q.i. and Q.p.

\begin{tabular}{|c|c|c|}
\hline $\begin{array}{c}\text { Tree-size } \\
\text { category } \\
\left(A_{x} \text { in } \mathrm{cm}^{2}\right) \\
\end{array}$ & $\begin{array}{c}\text { Average } J_{p i} \\
\text { for Q.i. } \\
\left(\mathrm{cm}^{3} \mathrm{~cm}^{-2} \mathrm{day}^{-1}\right)\end{array}$ & $\begin{array}{c}\text { Average } J_{p i} \\
\text { for Q.p. } \\
\left(\mathrm{cm}^{3} \mathrm{~cm}^{-2} \mathrm{day}^{-1}\right)\end{array}$ \\
\hline $\begin{array}{l}\text { “Small” } \\
(<200)\end{array}$ & 153.44 & 221.76 \\
\hline $\begin{array}{c}\text { "Medium" } \\
(\geq 200 \&<400)\end{array}$ & 173.72 & 275.04 \\
\hline $\begin{array}{c}\text { “Big” } \\
(\geq 400)\end{array}$ & 147.14 & 222.33 \\
\hline
\end{tabular}
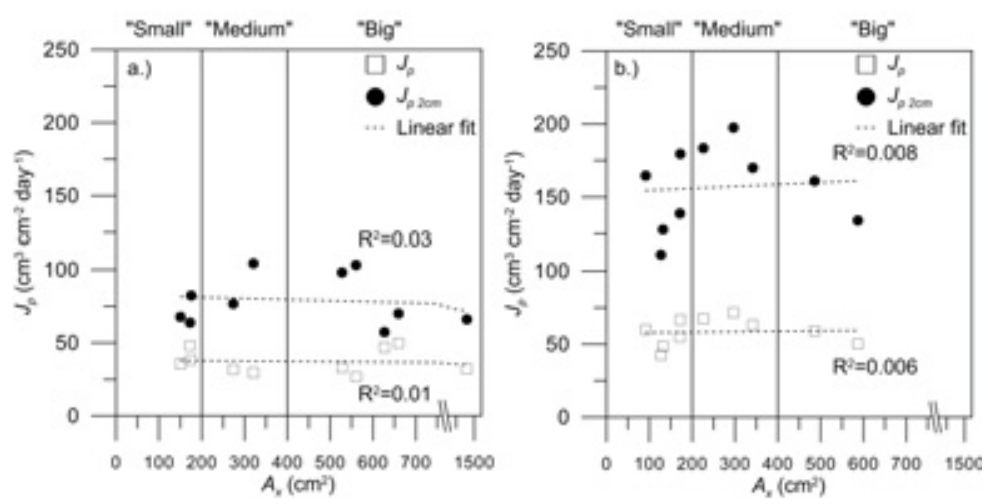

Figure 4.3: Variability of daily sap-flux density averaged across xylem depths $\left(J_{p}\right)$ and for $2 \mathrm{~cm}$ depth $\left(J_{p} 2 \mathrm{~cm}\right)$ in function of the the xylem area $\left(A_{x}\right)$ in a.) Q.i. and b.) Q.p.

\subsubsection{Modelling of temporal sap-flow variability}

The sap flow data obtained was limited to short campaigns of continuous daily measurements at 10 min intervals during different time periods of the dry season, due to technical and logistics limitations. To be able to provide transpiration maps throughout the dry season, it was therefore necessary to extrapolate temporally the available data. Several modelling techniques to simulate sap flow measurements have been reviewed, each 
with different levels of accuracy and complexity (Liu et al., 2009; O'Brien et al., 2004; Stöhr and Lösch, 2004; Verbeeck et al., 2007; Wheeler and Stroock, 2008; Whitley et al., 2009; Williams et al., 2001). We selected two modelling approaches that use typically measured climatic variables, namely: (1) a simple empirical micro-climatic model as described by Cienciala et al. (2000); and (2) Artificial Neural Networks (ANN) (Liu et al., 2009; Whitley et al., 2009). These two approaches were tested using customized scripts in MATLAB to choose the one that best simulated the available time-series data.

The empirical approach proposed by Cienciala et al. (2000) is based on combining the non-linear response of $Q_{s}$ to short-wave radiation and air relative-humidity, using the following equation:

$$
Q_{s}=\frac{a R_{s}}{\left[\left(c+R_{s}\right) \times(100-b \times R H)\right]}
$$

where $a, b, c$ are fitted parameters, $R_{s}$ is the short wave incoming solar radiation $\left(\mathrm{W} \mathrm{m}^{-2}\right)$ and $R H(\%)$ is the air relative-humidity. The parameters $a, b, c$ were first optimized to follow seven continuous days of $Q_{s}$ measurements of Q.i. and seven days of Q.p. in each tree sizecategory. Afterwards, the model was used to calculate $Q_{s}$ throughout the dry season to assess its accuracy as compared to the ANN.

The ANN is a purely statistically-based response of $Q_{s}$ to the meteorological forcing on a time-step basis (Abramowitz, 2005). The ANN was built and optimized as described by Liu et al. (2009), using seven non-continuous days of $Q_{s}$ measurements for training of the network. In our study, the ANN model included a vapour pressure deficit (VPD) of 2 $\mathrm{m}$ height, VPD of $6 \mathrm{~m}$ height, $R_{s}, R H$ and wind speed $\left(W_{s}\right)$ of $10 \mathrm{~m}$ height as the input variables (all normalized); $Q_{s}$ was the output variable. The soil moisture component was not used as it was the case in the study of Liu et al. (2009), because the $Q_{s}$ did not respond to the soil moisture dynamics during the dry season. The shallow water-table and the significant length of the roots of Q.i. and Q.p. trees, likely tapping groundwater, also explain the negligible response of $Q_{s}$ to soil moisture variability. Therefore, it was safe to assume that the dry season tree-transpiration in the Sardón catchment was driven mainly by the variation in climatic conditions.

The structure of the ANN models for simulating $Q_{s}$ in Q.i. and Q.p. consisted of three neural layers: an input layer, a hidden layer, and an output layer. The transfer functions used for the hidden and output layers are, hyperbolic-tangent-sigmoid transfer function (tan-sig) and logarithmic-sigmoid transfer function (log-sig), respectively, as described by Liu et al. (2009). After deriving Q.i. and Q.p. ANN models, they were used to calculate $Q_{s}$ throughout the entire dry season.

For validating both modelling techniques, the $Q_{s}$ outputs were compared to $Q_{s}$ measurements from 6 additional dry-season days (fulfilling dry-season standard) using the assessment procedure described by Ritter et al. (2011). They proposed to use the Nash Sutcliffe Efficiency (Ceff) 
index (McCuen et al., 2006) in a goodness-of-fit evaluation protocol referred as FITEVAL. The FITEVAL procedure is programmed in MATLAB and combines the calculation of various error indices to evaluate the performance of the model versus real $Q_{s}$ measurements, taking into consideration the effect of outliers and providing a probability density function to better discriminate the agreement between modelled and real $Q_{s}$. The final result is a probability distribution of the Ceff index typically between 0 and 1 ( 1 = best fit), ranked by four categories as follows: "Very Good" (Ceff $=0.9-1)$, "Good" (Ceff $=0.8-0.899)$, "Acceptable" $($ Ceff $=0.65-0.799)$ and "Unsatisfactory" $($ Ceff $<0.650)$.

\subsubsection{Remote sensing tree classification}

In the Sardón catchment there are two different oak tree species, Q.i. and Q.p., that had to be classified applying remote sensing technique. This was done by combining: (1) a supervised classification; (2) an objectoriented classification; and (3) a comparison of seasonal variation of the two tree species. The classification was applied on two satellite images of high spatial resolution (60 - $40 \mathrm{~cm}$ per pixel), acquired in two different seasons, namely: a QuickBird image during the 2009 (August) dry season and a WorldView-II image during the 2010 (December) wet season. Both satellite images were 4-bands multispectral, orthorectified, pan-sharpened and obtained with $0 \%$ cloud cover and a $<10^{\circ}$ nadir.

The first step of supervised classification using the 2009 QuickBird image of dry season did not yield sufficient accuracy. The second step of object-oriented classification improved the accuracy but still there was partial overlap of the electromagnetic spectra of Q.p. and Q.i. trees, in addition to their confusingly similar canopy shapes. Therefore, as Q.i. is an evergreen species and Q.p. is a deciduous species, an additional winter-season image was used to discriminate between leafless $Q . p$. and Q.i. The map obtained after the tree-species classification is referred below as the "classification map".

In the first step, the images were processed by a supervised classification using the ERDAS Imagine software to delineate the areas covered by tree canopies, as described by Ehlers et al. (2003). The supervised classification used the electromagnetic spectral values of the green, red and near infrared bands, which are sensitive to chlorophyll in vegetation (Janssen et al., 2001). The classification algorithms were trained by using the trees identified in the field survey (90 for Q.i. and 84 for Q.p.). The results of the supervised classification contained the tree canopy area coverage, but without distinction of species type at this stage.

In the second step of an object-oriented classification, spectral information and canopy shapes (object attributes) were used to discriminate between Q.i. or Q.p. species and to filter out other plants such as grasses and shrubs (Baatz et al., 2001; Dorren et al., 2003; Kimani et al., 2007). A multi-resolution segmentation algorithm and fine-tuning of the classification attributes (shape and compactness) was sufficient to define and delineate the tree canopies, thanks to the sharp contrast with the back- 
ground (granitic soil in open woodland). The classified canopies were further smoothed using a morphology algorithm to reshape odd canopy shapes. To apply the classification algorithm, the image was divided into tiles of 1 ha area, batch processed with the classification algorithm and finally merged back into one single image using eCognition developer 8.7 (Trimble Germany GmbH). Dividing the image into tiles for batchprocessing was necessary to reduce computing load and processing time. The object-oriented tree classification of the 2009 image, including both Q.i. and Q.p. species, resulted in an overall accuracy of $70 \%$. This level of accuracy was considered insufficient for the transpiration scaling-up exercise, and thus the second image during winter time (WorldView-II) was used to define the canopies of Q.i. only, as Q.p. had no leaves during this period. The object-oriented tree classification of the 2010 image was obtained using the same image processing as the 2009 image with overall accuracy of $\sim 95 \%$.

In the final step to achieve the highest possible classification accuracy, the results of the object-based classifications of the 2009 Quickbird dry-season image and of the 2010 WorldView-II wet-season image were cross-referenced using the spatial-selection tool of ArcMap 10 (ESRI, Inc.). In this way, the information about Q.i. in the 2010 map was transferred into the target classification map of the 2009. To execute the spatialselection tool, the 2009 classification map was used as the "target" to assign the Q.i. trees present in the 2010 classification map, which was defined as the "source". The re-classification of misclassified Q.i. tree canopies was carried out directly in the "Attribute Table" of the map. Likewise, Q.p. canopies misclassified as Q.i., and identified by their absence from the 2010 classification map, were re-assigned as well. The resulting vector layer was a tree-species classification map of $Q . i$. and Q.p. containing canopy areas classified per species for all the trees in the Sardón catchment. Such a classification map of Mediterranean Quercus sp. species can be considered as valid for several years because the change of the vegetation coverage in Mediterranean forests is naturally slow, a result of the dry inter-annual conditions that limit the growth rate (Corcuera et al., 2004; Hernández-Santana et al., 2009) and limited human intervention in the Sardón catchment (Lubczynski and Gurwin, 2005).

\subsubsection{Accuracy assessment of the tree classification}

To validate the tree-classification of Q.i. and Q.p., an accuracy assessment was applied as described by Congalton (1991). This accuracy assessment is based on the elaboration of an error matrix and the calculation of four accuracy indexes: (1) Overall accuracy, (2) User's accuracy, (3) Producer's accuracy, and (4) the Kappa coefficient. This assessment procedure has been used extensively with diverse types of land cover thematic maps (Giles M, 2002), and for tree-species classifications derived from remotely-sensed data (Carreiras et al., 2006; Kimani et al., 2007). 
To populate the error matrix, a large data set of ground-control points is needed (see Figure 4.2). Dividing the area into sample plots (Stehman and Czaplewski, 1998) and elaborating error matrices with ground-control points of those plots (Hay, 1979) is a common practice for the evaluation of land-cover maps (Giles M, 2002). We used a simple random sampling for the whole catchment (Stehman and Czaplewski, 1998), since conditions were quite uniform across the relatively small catchment $\left(\approx 80 \mathrm{~km}^{2}\right)$. 800 tree ground control points (128 for Q.i. and 672 for Q.p.) were selected randomly from trees located in 4 macro-plots established for the biometric survey (Reyes-Acosta and Lubczynski, 2014). Additional to those 800 trees, 208 trees were used in the training of the classification algorithms for both species. The 800 ground-control points were compared with the classification map using the spatial-join tool, and evaluated with a Boolean expression to quantify the matching records as follows: if the ground-control points species was the same as in the map classification, then the expression was $=1$, if false $=0$. The results of this procedure were used to fill the error matrix by totalizing the correctly classified and misclassified trees. Once the error matrix was filled (Table 4.3), the "Overall accuracy", "User's accuracy", "Producer's accuracy", and Kappa coefficient indexes were calculated using 800 ground-control points following the equations of Giles M (2002) as bellow:

$$
\text { Overall accuracy }=\frac{\sum_{i=1}^{q} n_{s s}}{n} \times 100 \%
$$

where "Overall accuracy" is a straightforward index of the overall accuracy in the analysed map layer, $q$ is the number of species categories, $s$ is a species-specific category index, $\sum_{i=1}^{q} n_{s s}$ is the sum of all correctly classified tree canopies, $n$ is the total number of ground-control points used in the assessment.

$$
\text { User's accuracy }=\frac{n_{s s}}{n_{s+}}
$$

where "User's accuracy" is a measure of commission error of the classified categories indicative of the probability that a classified canopy actually represents that species category on the ground (Story and Congalton, 1986); $s$ is a species-specific category index, $n_{s s}$ is the total number of correctly classified canopies in a species category; and $n_{s+}$ is the total number of classified canopies that were classified in that species-specific category (Congalton, 1991; Giles M, 2002).

$$
\text { Producer's accuracy }=\frac{n_{s s}}{n_{+s}}
$$

where "Producer's accuracy" is a measure of omission error of the classified categories, indicating the probability of a ground point to be correctly classified in a species-specific category $(s) ; n_{s s}$ is the total number of correctly classified canopies in a species category; and $n_{+s}$ is the 
total number of ground-recognised canopies that were classified in that species-specific category (Congalton, 1991; Giles M, 2002).

Additionally, the Kappa Index of Agreement (KIA) was calculated to account for the possibility of correctly classifying the tree canopies by random guessing (Cohen, 1960; Congalton, 1991; Giles M, 2002; Kimani et al., 2007). This is particularly relevant for the obtained tree classification in this study because in the Sardón catchment there are two tree species and one of them (Q.p.) is more common than the other (see Subsection 4.3.6), hence there is an inherited probability of properly classifying the tree canopies if all canopies were to be classified as the dominant one. The KIA is calculated as follows:

$$
K I A=\frac{n \times \sum_{i=1}^{q} n_{\mathrm{ss}}-\sum_{i=1}^{q}\left(n_{\mathrm{s}+} \times n_{+\mathrm{s}}\right)}{n^{2}-\sum_{i=1}^{q}\left(n_{\mathrm{s}+} \times n_{+\mathrm{s}}\right)}
$$

where, $q$ is the number of species-specific categories; $s$ is a speciesspecific category index; $n_{\mathrm{ss}}$ is the number of correctly classified canopies in a species category; $n_{\mathrm{s}+}$ and $n_{+\mathrm{s}}$ are the marginal totals of correctly classified canopies and ground-recognized canopies respectively, for each species-specific category; and $n$ is the total number of observations (Congalton and Green, 1999). When the obtained agreement is within the probability of a random classification the KIA value is zero, while 1 indicates $100 \%$ of agreement (Cohen, 1960). KIA values $>0.80(80 \%)$ indicate strong agreement, between 0.40 and $0.8(40-80 \%)$ moderate agreement and $<0.40$ (40\%) poor agreement (Congalton, 1996).

\subsubsection{Scaling up sap flow measurements for transpiration mapping}

In this study, the individual tree sap flow measurements $\left(Q_{s}\right)$ were considered to be equal to the amount of water transpired by a tree (Wullschleger et al., 1998; Lubczynski, 2009). Based on that assumption, $Q_{s}$ measurements were scaled up as described by Lubczynski (2009) using the following four elements: (1) a parameter to be scaled-up, (2) a scalar used for scaling-up, (3) a "Biometric Upscaling Function" (BUF), and (4) a scaling-up technique.

A scaling-up parameter (e.g. sapwood area $\left(A_{x}\right)$ or sap flow $\left(Q_{s}\right)$ ) is a parameter that is directly needed for quantifying transpiration but is difficult to define spatially. Its spatial variation can be defined (scaled up) by an easily measurable scaling-up scalar extrapolated using a BUF and an appropriate scaling-up technique. In this study we decided to use $A_{x}$ as the scaled-up parameter because, in contrast to $Q_{s}, A_{x}$ is nearly temporally independent so its scaling up is more reliable.

The selection of scalar depends on the scale of the transpiration assessment. In general, two different spatial scales of transpiration mapping can be considered: (i) plot or stand scale transpiration $\left(T_{s t a}\right)$; and (ii) catchment transpiration $\left(T_{c}\right)$. For $T_{s t a}$, stem areas $\left(A_{s}\right)$ are typically 
used as scaling-up scalars, because $A_{s}$ is known to be well-correlated with $A_{x}$ (Kumagai et al., 2005; Čermák et al., 2004) and can be measured from single trees at the plot or stand scale. At the catchment scale, such as the whole Sardón catchment $\left(\approx 80 \mathrm{~km}^{2}\right)$, it would not be practical and feasible to measure each single tree for $A_{s}$. Therefore, we used the canopy areas $\left(A_{c}\right)$ as the scalar instead, as the $A_{c}$ is retrievable from the high resolution images.

The third element necessary for scaling up sap flow measurements are the "Biometric Upscaling Functions" (BUF). The BUFs are field-defined, paired, species-specific relations between scalars and scaled parameters (Lubczynski, 2009). To properly select the BUF for Q.i. and Q.p., pairs of selected scalars $\left(D B H, A_{s}\right.$ and $\left.A_{c}\right)$ and scaled parameters $\left(A_{x}\right)$ were evaluated with a Pearson product-moment correlation-coefficient $(r)$ matrix (Quinn and Keough, 2002) and then regressions were calculated and described by the coefficient of determination $\left(R^{2}\right)$. These coefficients evaluate the potential of the parameters (in this case a scaling-up scalar) to predict the others (in this case scaling-up parameters) using the proposed regressions (Quinn and Keough, 2002). The result of the analysis was used to determine the highest $R^{2}$ from the selected pairs of parameters (i.e. $D B H$ vs. $A_{x}$ and $A_{c}$ vs. $A_{x}$ ), thus highlighting the best linear or polynomial models from the pairs of parameters to be used as BUFs. To properly cover the natural variability of the scalars and scaled-up parameters 90 Q.i. and 84 Q.p. tree samples were used to derive the BUFs. This sample size guaranteed that the natural variability of the biometric characteristics was properly accounted for as discussed in Reyes-Acosta and Lubczynski (2014).

The fourth element necessary for the scaling-up process was the scaling-up technique. For this study, we selected remote sensing as the scaling up technique because: $(1)$ the size of the Sardón catchment $(\approx 80$ $\mathrm{km}^{2}$ ) did not permit an in-situ assessment of tree transpiration; (2) the remote-sensing technique allowed for an automated classification of the tree species and an efficient measurement of the canopy areas for the whole catchment (the scalar in this study); (3) the Sardón catchment is covered by open woodland, which facilitates the definition of individual tree canopies, particularly when using high-resolution remotely-sensed images; (4) tree-species classification was relatively simple due to the twospecies composition (one deciduous, one evergreen) of the vegetation in the study area, which resulted in a highly accurate classification; and finally (5) the high $R^{2}$ for the BUFs indicated a good prediction potential.

The methodology of transpiration mapping at the catchment scale $\left(T_{\mathcal{C}}\right)$ followed the series of steps depicted in Figure 4.4. First, after classifying the tree canopies per species, the projected areas of the canopies $\left(A_{c}\right)$ were automatically calculated using GIS tools from the classification map. For those cases in which several trees were naturally arranged in one cluster, they were considered as a single larger tree with biometric characteristics equal to the sum of the biometric parameters of the individual trees. Second, species-specific BUFs were established. Third, using the BUFs of Q.i. and Q.p., the scaled up $A_{x}$ was calculated for all 


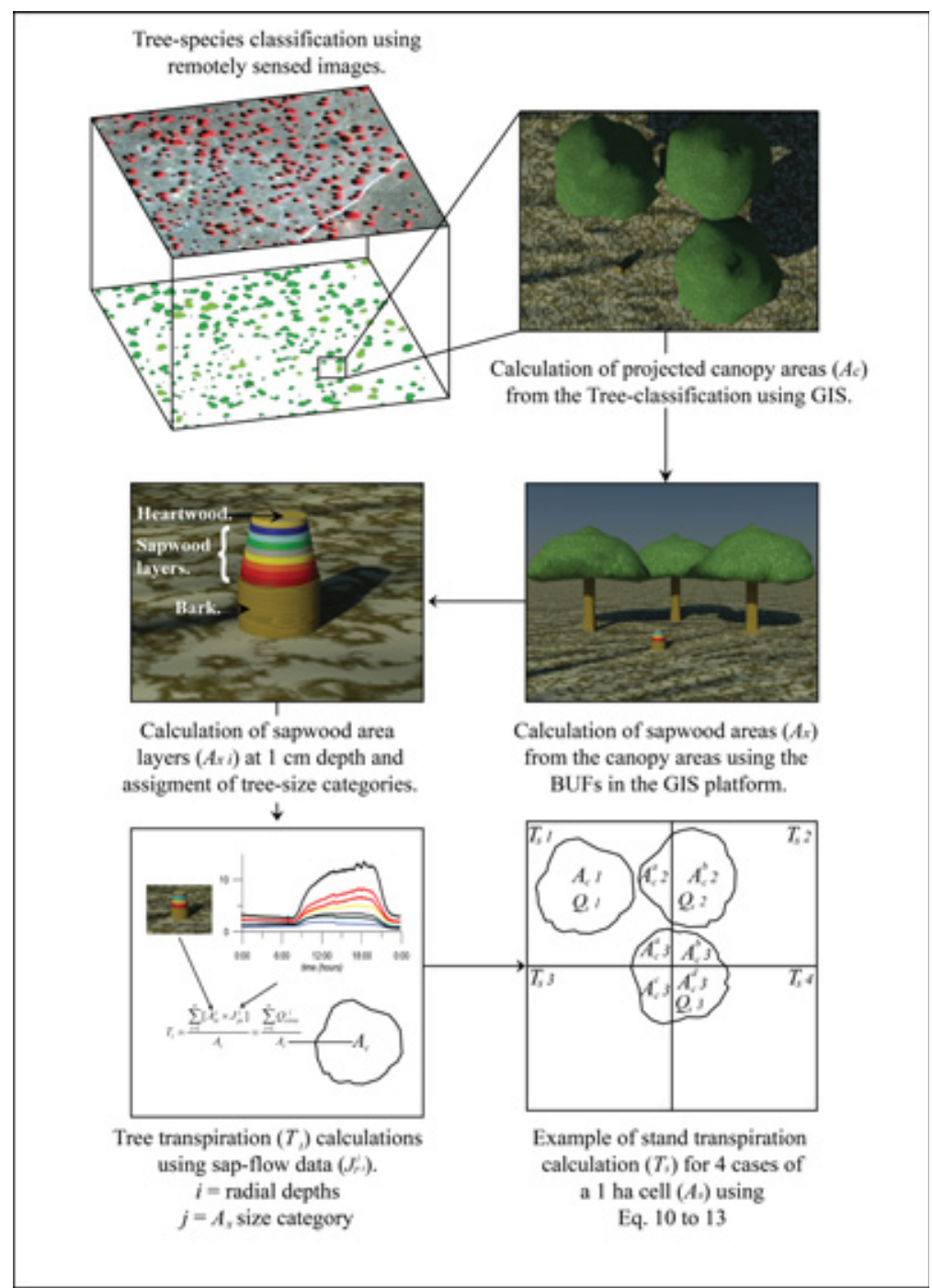

Figure 4.4: Summary of the main steps of tree transpiration up-scaling from remotely sensed imagery and GIS techniques.

the trees in the entire catchment. Fourth, using geometric equations, the $A_{x}$ areas of all the trees were divided into annuli areas (ring-like) representing concentric sapwood areas $\left(A_{x i}\right)$ of $1 \mathrm{~cm}$ thickness (Figure 4.4). This was done to take into consideration the radial variability of the sap flux density $\left(J_{p-i}\right)$ obtained by optimised $J_{p-i}$ measurements as presented by Reyes-Acosta and Lubczynski (2014). Fifth, $Q_{s}$ of individual trees was calculated as a sum of $Q_{s i}^{s c}$ of each individual annulus per 
tree-size category, as follows:

$$
Q_{s}=\sum_{i=1}^{n} Q_{s i}^{s c}=\sum_{i=1}^{n}\left[A_{x i} \times J_{p i}^{s c}\right] \quad\left[l d a y^{-1}\right]
$$

where each $Q_{s i}^{s c}$ is defined as a product of sapwood area $\left(A_{x i}\right)$ and $i$ - th sap-flux density $\left(J_{p i}^{s c}\right)$ of the corresponding sapwood annulus for each tree-size category $(s c)$. The subscript $n$ is the total number of annuli. In the case of trees with a sapwood depth $>7.5 \mathrm{~cm}$ (max. depth of HFD), it was assumed that the rest of the sapwood transported a linearly decreasing with depth amount of sap flow until it became zero in the heartwood. Sixth, to obtain the canopy-normalised sap flow, also referred as canopy transpiration $\left(T_{t}\right)$, each $Q_{s}$ was divided by the area of its own canopy to obtain $T_{t-Q i}$ and $T_{t-Q p}$ as follows:

$$
T_{t}=\frac{Q_{s}}{A_{c}}=J_{p} \times \frac{A_{x}}{A_{c}} \quad\left[m m d a y^{-1}\right]
$$

Seventh, to calculate the transpiration of a stand, the sum of all tree water-uptakes in a stand $\left(\sum_{j=1}^{n} Q_{s j}\right)$ was divided by the corresponding stand area $\left(A_{\text {sta }}\right)$, as in Equation 4.9. The sub-index $j$ refers to each single tree in the stand and $m$ to the number of trees in the stand.

$$
T_{\text {sta }}=\frac{\sum_{j=1}^{m} Q_{s j}}{A_{\text {sta }}}\left[\mathrm{mmday}^{-1}\right]
$$

For this study, we have chosen an area of 1 ha for the analysis of the stands, thus the catchment area was discretised using a 1 ha grid. To calculate $T_{\text {sta }}$ for each grid in the catchment, the tree-classification map was first segmented by the grid using the spatial-join tool of ArcMap 10. Then the $Q_{s j}$ of each segmented canopy was summed for each cell in the grid and divided by the area of a single cell of the grid $\left(A_{\text {sta }}=1 \mathrm{ha}\right)$ to calculate $T_{\text {sta }}$. For the relative-contribution of canopies that crossed cell boundaries, a canopy fractions approach was applied to calculate $T_{\text {sta. }}$. An example of three different cases of cells' boundaries overlap can be seen in Figure 4.4, namely: (1) the contribution of single canopy segments $\left(A_{c 1}^{c}\right.$ and $\left.A_{c 1}^{d}\right)$, each one in a different cell (defined as $T_{s t a 1}$ and $T_{\text {sta } 2}$, respectively); (2) the contribution of two canopy segments $\left(A_{c 2}^{b}\right.$ and $A_{c 1}^{b}$ ) from two separate trees in one cell (defined as $T_{\text {sta } 3}$ ); and (3) the contribution of a full canopy $\left(A_{c 4}\right)$ together with canopy segments of two separate trees $\left(A_{c 2}^{a}\right.$ and $\left.A_{c 1}^{a}\right)$ inside one cell (defined as $\left.T_{\text {sta }}\right)$. For each case, Equation 4.9 was transformed to fit the arrangement of the segments in each cell, thus obtaining Equations 4.10 - 4.12. For $T_{\text {sta } 1}$ and $T_{\text {sta } 2}$, Equation 4.10 was used, for $T_{\text {sta } 3}$, Equation 4.11 was used, and 
for $T_{\text {sta } 4}$ Equation 4.12 was used. $A_{c 1}, A_{c 2}$, and $A_{c 3}$ define the entire canopy area of each tree.

$$
\begin{aligned}
& T_{s t a 1}=\frac{Q_{s 1} \frac{A_{c 1}^{c}}{A_{c 1}}}{A_{s t a}} \quad \text { and } T_{s t a 2}=\frac{Q_{s 1} \frac{A_{c 1}^{d}}{A_{c 1}}}{A_{s t a}} \\
& T_{\text {sta } 3}=\frac{Q_{s 2} \frac{A_{c 2}^{b}}{A_{c 2}}}{A_{s t a}}+\frac{Q_{s 1} \frac{A_{c 1}^{b}}{A_{c 1}}}{A_{s t a}} \\
& T_{s t a 4}=\frac{Q_{s 1} \frac{A_{c 1}^{a}}{A_{c 1}}}{A_{s t a}}+\frac{Q_{s} 2 \frac{A_{c 2}^{a}}{A_{c 2}}}{A_{s t a}}+\frac{Q_{s 3} 3 \frac{A_{c 3}}{A_{c 3}}}{A_{s t a}}
\end{aligned}
$$

After processing, all cells in the map using Equations 4.10 - 4.12, the resultant map contained hectare-specific values of transpiration in 1 ha quadratic grid expressed in $\mathrm{mm} \mathrm{day}^{-1}\left(T_{\text {sta }}\right)$. To calculate transpiration at the catchment level as a total $\left(T_{c}\right)$, an average $T_{\text {sta }}$ of all grid cells was calculated.

\subsubsection{Assessment of remote sensing scaling-up procedure}

In order to evaluate the performance of scaling up using remote sensing, we compared it to in-situ stand-scale (i.e. plot) scaling up followed by an uncertainty analysis. For that purpose, we selected a 1 ha stand $(100 \mathrm{~m} \times$ $100 \mathrm{~m}$ ) in which all trees in that stand (7 Q.i. and 6 Q.p.) were measured and the scaling-up procedure performed using a BUF relating $D B H$ with $A_{x}$. For the remote-sensing scaling up of the same plot, the BUF relating $A_{c}$ (as obtained by the attributes of the classified canopies) with $A_{x}$ was used.

For the uncertainty analysis we applied a Monte-Carlo simulation using the probability distributions of the error associated with two of the most important steps of the remote sensing scaling up, namely: (1) the uncertainty from misclassified classes (Q.i. or Q.p., respectively) in the tree classification map, and (2) the uncertainty in the confidence interval of the derived BUFs.

Additional factors such as installation errors, equipment malfunctioning and/or limitations, natural variations in the sapwood tissue, etc. also influence the accuracy of sap flow measurements. However, to account for all those factors is very complex, which was outside the scope of this research. Nevertheless, the optimisation technique used to acquire the sap flow measurements was designed to enhance the accuracy and reduce the most important biasing factors to a minimum, thus it was assumed that the sap flow measurements in this study have the highest accuracy possible and that the error is negligible.

To properly define the error for the species classification we used a random binomial distribution defined by the probability calculated in the 
"User" accuracy assessment. Using a binomial distribution is justified because the error probability for the classification maps is limited to just two species. To account for the error of the BUFs, we used a random normal distribution derived from a 99\% confidence interval, because the distribution of the norm of residuals of a least-squares linear fit is assumed to follow a normal distribution (Rice, 2006).

The Monte-Carlo simulations were run for 1000 iterations to reach a satisfactory convergence of the tree transpiration calculations. For each iteration, the random binomial distribution was first simulated and then followed by the random normal distribution simulation. At the end of each iteration, $T_{c}$ was calculated according to the equations given in the Subsection 4.2.7, using as input the random species classification and the species-specific random $A_{x}$ values of each BUF (for Q.i. and Q.p.). After 1000 iterations, the distributions of the outputs were examined.

\subsection{Results and Discussion}

\subsubsection{Tree biometric characteristics}

The frequency histograms of the biometric characteristics of 90 Q.i. trees and 84 Q.p. trees (diagonals in Figure 4.5) followed a quasi-normal distribution skewed to the left. The distribution of the biometric parameters indicated that the tree population for both species was mostly composed of small - to medium - tree-stem sizes $\left(D B H<0.5 \mathrm{~m}\right.$ and $\left.A_{s}<0.25 \mathrm{~m}^{2}\right)$, with small - to medium canopy areas $\left(A_{c}<50 \mathrm{~m}^{2}\right)$ (Table 4.2) and small xylem areas $\left(A_{x}<0.05 \mathrm{~m}^{2}\right)$. The trees with small - to medium-size $D B H$, held more than $90 \%$ of the total sapwood tissue for both tree populations. Big trees with large canopies $\left(>100 \mathrm{~m}^{2}\right)$ and big trunk sizes $(>0.70 \mathrm{~m})$ were rare and distinct but due to their scarcity they were less significant as contributors to the catchment's tree transpiration.

Table 4.2: Main statistics of the biometric parameters obtained in the survey for Q.i. $(n=90)$ and Q.p. $(n=84)$

\begin{tabular}{llllllllllll}
\hline & \multicolumn{3}{c}{$D B H(\mathrm{~m})$} & \multicolumn{2}{c}{$A_{t}\left(\mathrm{~m}^{2}\right)$} & \multicolumn{2}{c}{$A_{c}\left(\mathrm{~m}^{2}\right)$} & \multicolumn{2}{c}{$A_{s}\left(\mathrm{~m}^{2}\right)$} & \multicolumn{3}{c}{$A_{x}\left(\mathrm{~m}^{2}\right)$} \\
\cline { 2 - 11 } & Q.i. & Q.p. & Q.i. & Q.p. & Q.i. & Q.p. & Q.i. & Q.p. & Q.i. & Q.p. \\
\hline Min. & 0.11 & 0.14 & 0.01 & 0.02 & 9.88 & 10.02 & 0.01 & 0.02 & 0.01 & 0.01 \\
Max. & 0.97 & 1.05 & 0.74 & 0.87 & 146.3 & 141.0 & 0.74 & 0.87 & 0.14 & 0.11 \\
Mean & 0.34 & 0.32 & 0.12 & 0.10 & 43.46 & 43.67 & 0.12 & 0.10 & 0.05 & 0.03 \\
SD & 0.20 & 0.17 & 0.15 & 0.13 & 30.73 & 25.66 & 0.15 & 0.13 & 0.03 & 0.02 \\
Median & 0.28 & 0.28 & 0.06 & 0.06 & 35.26 & 38.88 & 0.06 & 0.06 & 0.04 & 0.03 \\
\hline
\end{tabular}




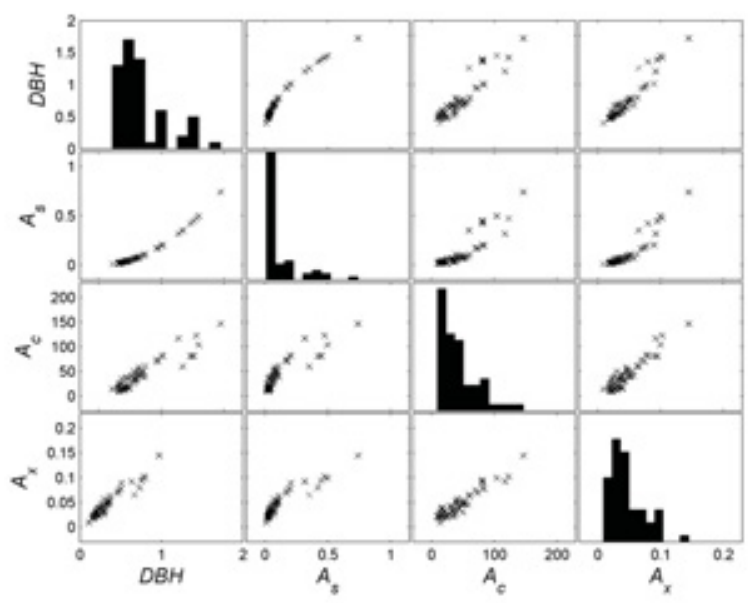

a

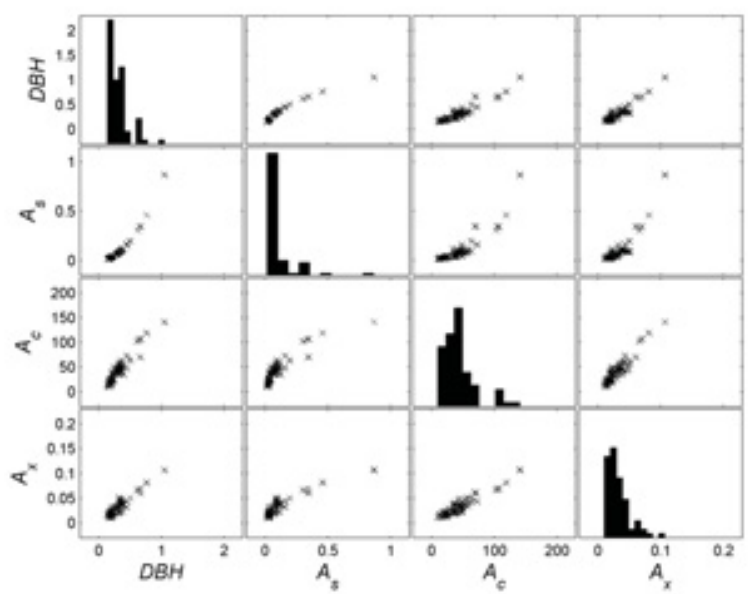

b

Figure 4.5: Cross-correlation matrix of the biometric parameters measured in the survey for (a) Q.i. and (b) Q.p. trees. Diameter at breast height $(D B H)$ is in $\mathrm{m}$, area of the stem $\left(A_{s}\right)$ is in $\mathrm{m}^{2}$, ground-projected canopy area $\left(A_{\mathcal{C}}\right)$ is in $\mathrm{m}^{2}$, and xylem area $\left(A_{x}\right)$ is in $\mathrm{m}^{2}$.

\subsection{2 sap flow patterns for Q.i. and Q.p.}

$J_{p}$ measurements used in this study are described in Reyes-Acosta and Lubczynski (2014). For the proposed scaling up procedure, the relation between $A_{x}$ and $J_{p}$ is critical. Linearity tests of average $J_{p-i}$ at 0.5 $\mathrm{cm}$ and $1.5 \mathrm{~cm}$ depths in the sapwood $\left(J_{p 2 \mathrm{~cm}}\right)$ and average $J_{p-i}$ for all sapwood annuli $\left(J_{p}\right)$, from 10 Q.i. and 10 Q.p. trees, indicated a very low 
correlation with $A_{x}$ for Q.i. $\left(R^{2}=0.03\right.$ for $J_{p 2 c m}$ and $R^{2}=0.01$ for $\left.J_{p}\right)$ and Q.p. $\left(R^{2}=0.008\right.$ for $J_{p 2 c m}$ and $R^{2}=0.006$ for $\left.J_{p}\right)$, indicating no significant dependence between $A_{x}$ and $J_{p}$. Instead, as seen in Figure 4.3 for Q.i. and Q.p., $J_{p 2 \mathrm{~cm}}$ depicted an increment towards the "medium" $A_{x}$ values and then decreased again towards the "large" $A_{x}$ values (Figure 4.3). Such pattern was not present in the relation of $J_{p}$ (average sap-flux density across the sapwood profile) with $A_{x}$. The described $J_{p 2 \mathrm{~cm}}-A_{x}$ pattern implied that the largest $J_{p 2 \mathrm{~cm}}$ occurred in the trees of the medium size.

Considering that the outermost $2 \mathrm{~cm}$ of the Q.i. and Q.p. sapwood typically conducted most of $Q_{s}$ (Reyes-Acosta and Lubczynski, 2014), not only due to the largest $J_{p}$, but also due to the largest corresponding annuli sapwood areas (external sapwood rings are the largest), and that the $J_{p 2 c m}$ significantly varied with $A_{x}$, a categorization of the sap flux density measurements as dependent on $A_{x}$ was necessary. Even though the differences between $J_{p 2 c m}$ as presented in Figure 4.3 were low, they were significant because of the multiplicative effect of the outermost sapwood layer, implying that small differences of $J_{p 2 c m}$, resulted in large differences of $Q_{s}$.

It is important to clarify that boundaries for the size-categories were intentionally set at different thresholds for both species, Q.i. and Q.p., in order to better reflect the variability of the sap-flux density as dependent on the tree population structures. As a result, the derived $A_{x}$ categories of sap flux density (Figure 4.3) also listed in Table 4.1, have species-specific $A_{x}$ ranges, except for the category "large" with common threshold $\geq 400 \mathrm{~cm}^{2}$. Considering $J_{p 2 \mathrm{~cm}}$ as reference for categorization, the highest average values in both species belonged to the "medium" category and were $101.9 \mathrm{~cm}^{3} \mathrm{~cm}^{-2}$ day $^{-1}$ for Q.i. and $179.3 \mathrm{~cm}^{3} \mathrm{~cm}^{-2}$ $\mathrm{day}^{-1}$ for Q.p. trees. For the categories "small" and "large", the $J_{p 2 \mathrm{~cm}}$ was significantly lower (Table 4.1).

It seems that such $A_{x}-J_{p 2 c m}$ distribution is not uncommon. For example Chavarro-Rincón (2009) in savannah trees of the Kalahari and Köstner et al. (1998) in a Scots pine plantation also found that the largest $J_{p 2 c m}$ was attributed to medium size trees $A_{x}-J_{p 2 c m}$ distribution. The presented $J_{p 2 c m}$ categorization substantially improves the accuracy of scaling of sap flow measurements. After confirming variable $A_{x}$ $J_{p 2 \mathrm{~cm}}$ distribution (for example with HFD) that can be categorised, we recommend to use the categorisation of $J_{p 2 c m}$ as proposed in this study.

The results from the sap flow field measurements showed that both $J_{p 2 c m}$ and $J_{p}$ were higher in Q.p. than in Q.i. trees for all categories (Table 4.1), while $Q . i$. trees tended to be more active in deeper sapwood layers (5-7 cm deep) than Q.p. trees (active until $4 \mathrm{~cm}$ deep) (Reyes-Acosta and Lubczynski, 2014). These results imply that individual Q.p. trees of the same $A_{x}$ as $Q . i$. contributed more to the water uptake. An interesting observation was made that the Q.i. and Q.p. species had relatively low variability of $J_{p}$ around their means (Figure 4.3), confirmed by low standard deviation (Table 4.1). This low variability suggests that growing Q.i. and Q.p. trees increase their water uptake $\left(Q_{s}\right)$ proportionally to $A_{x}$ lso to $A_{s}$ that increases with tree growth. 


\subsubsection{Temporal extrapolation of sap flow measurements}

The dry-season temporal extrapolation using the "Cienciala Model" (CM) and Artificial Neural Networks (ANN) was carried out in 3 months period of 2009 (from 15 June to 15 September), to assess the dry-season temporal variability of sap flow and tree transpiration in that period.

Evaluation of the performance of the CM and ANN showed that the goodness-of-fit of the $Q_{s}$ simulations using the ANN method was "very good" for Q.i. (Ceff between 0.92 and 0.96) and Q.p. (Ceff between 0.97 and 0.99 ) (Figure 4.6). Significantly lower accuracy was obtained by applying CM. For Q.i. the Ceff was between 0.75 and 0.85 , indicating a goodness-of-fit between "acceptable" and "good", while for Q.p. the Ceff was between -0.60 and -0.11 , indicating an "unsatisfactory" goodness-offit (Figure 4.6). This was mainly because the night flow of Q.p. trees was not taken in consideration by the CM model. The better performance of ANN as compared to CM was related to the use of more climatic drivers as inputs and the greater flexibility of the model. For the ANN method we used the vapour-pressure deficit at two heights $\left(V P D_{2 m}\right.$ and $\left.V P D_{6 m}\right)$ and wind speed $\left(W_{s}\right)$ at $10 \mathrm{~m}$ height, while in CM only incoming solar radiation and relative humidity at $2 \mathrm{~m}$ height were used. The additional climatic drivers used for the ANN are closely linked to the transpiration dynamics (Chu et al., 2009; Zeppel et al., 2004) and constrained the ANN sap flow calculations. Consequently, this is why the ANN approach was selected to simulate dry-season sap flow dynamics for both Q.i. and Q.p. species (Figure 4.7a).

The daily means and maxima $Q_{s}$ obtained from the ANN-simulations showed a low temporal variability for both $Q . i$. and Q.p. trees (Figure 4.7a). This was indicated by the low standard deviation (SD) between daily $Q_{s}$ means (for $Q . i$. the mean of daily means was $825 \mathrm{~cm}^{3} \mathrm{~h}^{-1}$ with $\mathrm{SD}=151 \mathrm{~cm}^{3} \mathrm{~h}^{-1}$ and for Q.p. $1611 \mathrm{~cm}^{3} \mathrm{~h}^{-1}$ with $\left.\mathrm{SD}=151 \mathrm{~cm}^{3} \mathrm{~h}^{-1}\right)$. The overall good match between the modelled and measured transpiration including good agreement with respect to the low temporal variability of daily $Q_{s}$ means and maxima (Reyes-Acosta and Lubczynski, 2014), imply that the dry-season therefore transpiration values scaled up from the dry-season standardised $J_{p}$ data, can be assumed to be representative for most of days during the dry season (July to September), i.e. for those days fulfilling the criteria of the "standard" dry-season day (see Subsection 4.2.3 for details on $J_{p}$ standardisation).

The only considerable differences in $Q_{s}$ temporal variability were noticed in some few days during dry season when some clouds and rain were present (Figure $4.7 \mathrm{a}-\mathrm{b})$. These days were indicated by a considerably higher SD of $Q_{s}$ (e.g. $400 \mathrm{~cm}^{3} \mathrm{~h}^{-1}$ for Q.i. and $300 \mathrm{~cm}^{3} \mathrm{~h}^{-1}$ for Q.p.). For such days the simulated $Q_{s}$ was lower than measured. However these episodes were rare during the dry season, thus the scaling up estimations from standardized non-cloudy measurements represent the dominant dry-season transpiration in the Sardón catchment. In case of missing records in time series of sap flow measurements, the ANN modelling framework can be used to simulate the missing measurements 

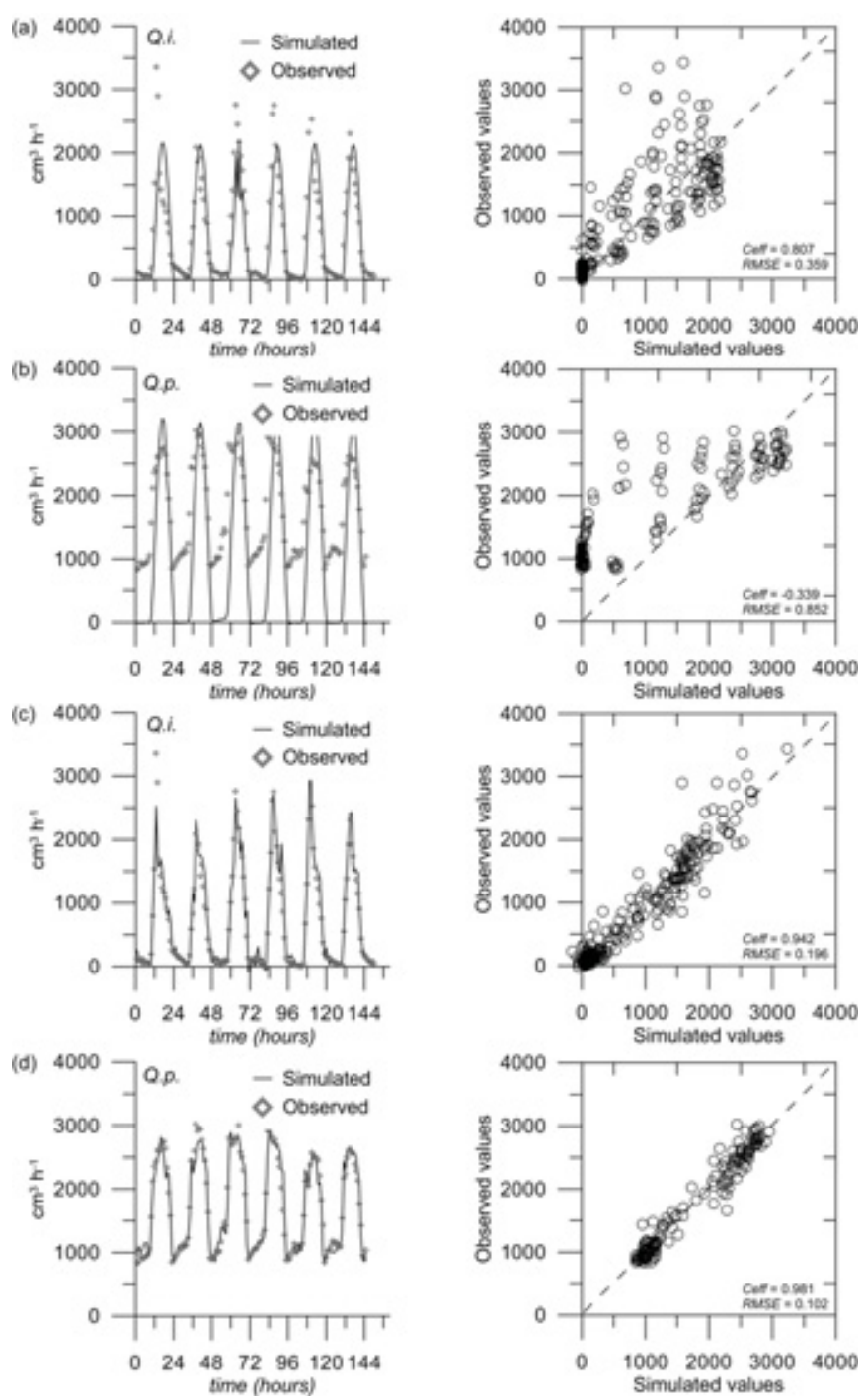

Figure 4.6: FITEVAL assessment of the sap-flow simulation results for (a) Cienciala model, Q.i.; (b) Cienciala model, Q.p.; (c) ANN model, Q.i.; (d) ANN model, Q.p.

and consequently calculate catchment transpiration in any conditions, assuming that the ANN framework can reflect the effect of cloudy-rainy conditions in the sap flow. Unfortunately, in this study was not possible to validate this assumption because there was no opportunity to monitor sap flow on those very few cloudy-rainy days in dry season. 


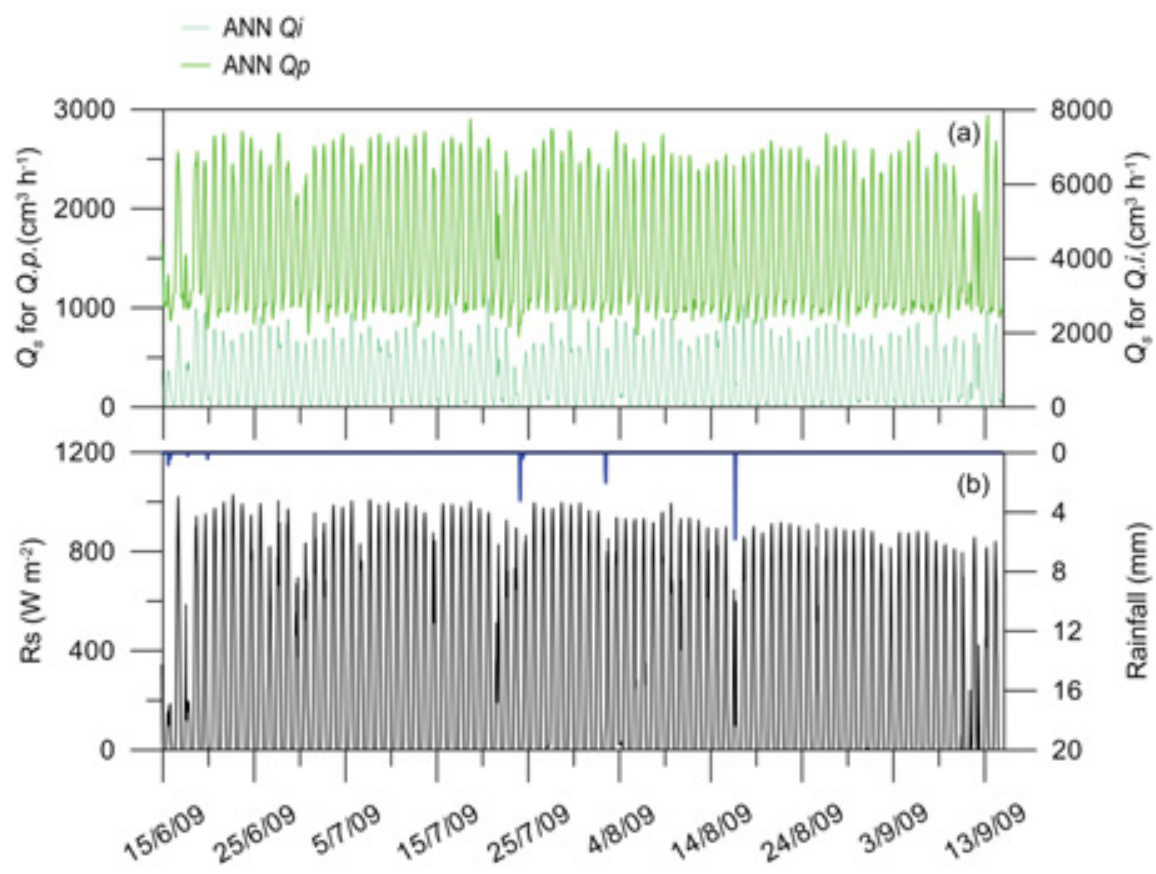

Figure 4.7: Dry season temporal sap flow versus climatic temporal variability: (a) daily ANN sap-flow simulations during the dry season of 2009 (15 June - 15 September) for Q.i. and Q.p. trees; (b) incoming solar radiation $(R s)$ and rainfall.

\subsubsection{Accuracy of the tree classification}

Accurate tree classification is an important component of the sap-flow scaling-up method, particularly when trees of different species but the same $A_{c}$ have significantly different $Q_{s}$. In our study the two tree species, Q.i. and Q.p., differed in dry-season sap flow.

The "Overall accuracy" (Equation 4.3) of the tree classification map was $90 \%$, which is the probability that any randomly-chosen tree canopy in the classification map was correctly assigned. Regarding the other accuracy indexes (as calculated by using the error matrix of misclassified trees in Table 4.3), it was found that the "User's accuracy" (Equation 4.4) for a randomly chosen $Q . i$. canopy in the map had a higher probability (96\%) of being properly classified than the Q.p. canopy (92\%). Conversely, "Producer's accuracy" (Equation 4.5) indicated that a Q.p. canopy randomly chosen in the field had a higher probability (90\%) of being properly classified than a Q.i. canopy (68\%). These indexes indicated that the accuracy of the classification map for each species was sufficient and that it was dependent on the number of trees of each species. The average, catchment scale tree density for Q.p. was higher (19 trees $\left.h a^{-1}\right)$ than for $Q . i$. (4 trees $h a^{-1}$ ), thus increasing the probability of matching Q.p. 
ground control points with the classification map, but also reducing the probability of successfully classifying all the Q.i. trees in the field.

Table 4.3: Error matrix of 800 randomly selected ground-control points (canopies) used for the calculation of the error indexes of the tree-species classification map.

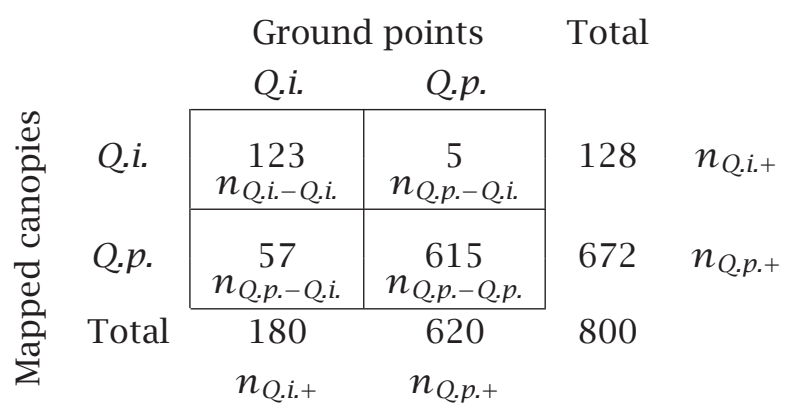

The final accuracy index, the KIA (Equation 4.6), was 0.8. According to the qualitative scale for KIA values proposed by Congalton (1996), the obtained KIA value indicated strong agreement between the ground control points and the classification map, with a low probability of correctly classifying tree canopies by random guess alone. This further supported the validity of the classification map and its robustness for the scaling-up process.

Despite the difference found between the accuracies for each species, the accuracy assessment indexes indicated that the tree classification map was accurate, thus any misclassification of canopies in the map would have a minimal effect on the scaled-up transpiration calculations. This effect of misclassification is discussed further in Subsection 4.3.8.

\subsubsection{Scalars, scaling-up parameters and BUFs}

A preliminary assessment of the biometric characteristics using the Pearson product-moment correlation-coefficient matrix $(r)$ showed a significant interdependence for various combinations of characteristics of both species (Figure 4.5 and Table 4.4). $A_{x}, A_{c}, A_{s}$ and $D B H$ gave high $r$ values for both species, indicating a strong interdependency and good potential for building a robust and precise BUF.

To establish the BUFs for Q.i. and Q.p. trees, $A_{x}$ was selected as the scaled-up parameter because of its low temporal variability. $A_{c}$ was selected as a remote-sensing-based scalar because it can be accurately defined on remotely-sensed images of very high spatial resolution, and, finally, $D B H$ as the stand scaling-up scalar because it is easy to measure in the field. The $D B H-A_{x}$ of both species fitted a second grade polynomial equation, which resulted in $R^{2}=0.88$ for $Q$.i. trees (Figure $4.8 \mathrm{a}$ ) and $R^{2}=0.91$ for Q.p. trees (Figure 4.8c). The $A_{c}-A_{x}$ BUFs of both species fitted a linear equation, with the coefficients of determination $R^{2}=0.84$ for Q.i. trees (Figure 4.8b) and $R^{2}=0.86$ for Q.p. trees (Figure 4.8d). 
It is remarkable that although the differences between the BUFs of $Q . i$. and $Q . p$. were not large (Figure 4.8), both BUF types, i.e. DBH- $A_{x}$ and $A_{c}-A_{x}$ of $Q . i$. were steeper, having larger $A_{x} / A_{c}$ than Q.p. indicating faster increase of $A_{x}$ with the increase of $D B H$ and $A_{c}$. This means that a canopy area recognized for example by remote sensing as $Q . i$. would have larger $A_{\mathcal{x}}$ than the same size canopy area of Q.p.

Table 4.4: Pearson product-moment correlation coefficient $(r)$ matrix for the surveyed biometric parameters in Q.i. and Q.p.

\begin{tabular}{ccccccc}
\hline \multicolumn{7}{c}{ Q.i. } \\
\hline & $D B H$ & $A_{t}$ & $T_{h}$ & $A_{c}$ & $l_{s}$ & $A_{x}$ \\
\hline$D B H$ & 1 & 0.98 & 0.66 & 0.87 & -0.06 & 0.86 \\
$A_{t}$ & 0.98 & 1 & 0.59 & 0.84 & -0.09 & 0.83 \\
$T_{h}$ & 0.66 & 0.59 & 1 & 0.63 & 0.02 & 0.61 \\
$A_{c}$ & 0.87 & 0.84 & 0.63 & 1 & 0.16 & 0.86 \\
$l_{s}$ & -0.06 & -0.09 & 0.02 & 0.16 & 1 & 0.43 \\
$A_{x}$ & 0.86 & 0.83 & 0.61 & 0.86 & 0.43 & 1 \\
\hline
\end{tabular}

Min. $r \quad-0.09$

Max. $r \quad 0.98$

Mean $r \quad 0.55$

Stdv. $r \quad 0.37$

\begin{tabular}{ccccccc}
\hline \multicolumn{7}{c}{ Q.p. } \\
\hline & $D B H$ & $A_{t}$ & $T_{h}$ & $A_{c}$ & $l_{s}$ & $A_{x}$ \\
\hline$D B H$ & 1 & 0.97 & 0.67 & 0.95 & -0.48 & 0.97 \\
$A_{t}$ & 0.97 & 1 & 0.56 & 0.89 & -0.41 & 0.93 \\
$T_{h}$ & 0.67 & 0.56 & 1 & 0.76 & -0.53 & 0.65 \\
$A_{c}$ & 0.95 & 0.89 & 0.76 & 1 & -0.50 & 0.95 \\
$l_{s}$ & -0.48 & -0.41 & -0.53 & -0.50 & 1 & -0.35 \\
$A_{x}$ & 0.97 & 0.93 & 0.65 & 0.95 & -0.35 & 1 \\
\hline
\end{tabular}

Min. $r \quad-0.53$

Max. $r \quad 0.97$

Mean $r \quad 0.40$

Stdv. $r \quad 0.64$

The $R^{2}$ coefficients showed that using $D B H$ as the scalar, yielded the best fits for both species, although using $A_{c}$ as the scalar also provided high $R^{2}$, indicating a reliable BUF. The performance of both scaling-up techniques are compared and discussed in Subsection 4.3.7. Lubczynski and Gurwin (2005) also used $A_{c}$ as a scalar and $A_{x}$ as a scaling-up parameter in the same Sardón-catchment study area, but they obtained a much lower $R^{2}$ for both species (0.62), and their linear equations showed a higher proportion of $A_{x}$ per unit of $A_{c}$ (0.0022 for Q.i. trees and 0.0026 for Q.p. trees), than ours (0.0008 for Q.i. trees and 0.0007 for 
Q.p. trees). This discrepancy is likely to be related to the sample size, because Lubczynski and Gurwin (2005) sampled 22 Q.i. and 25 O.p. trees, whereas we sampled 90 Q.i. and 84 Q.p. trees. Besides, Reyes-Acosta and Lubczynski (2014) have demonstrated that the $D B H$ size variability for Q.i. and Q.p. trees stabilizes after random sampling of more than 60 trees. A larger sample size gives better estimates of the characteristics of Q.i. and Q.p. tree populations, particularly for the characteristics of big trees $(D B H>0.5 \mathrm{~m})$, which greatly influence the BUFs' linear relations (Figure 4.8). For future scaling-up studies on other species in open woodlands, we advise using a sampling design that effectively covers the natural variability of the trees (in our case $>60$ trees), such as stratified sampling (Reyes-Acosta and Lubczynski, 2014) or quartiles of the total (Čermák et al., 2004).

The analysis in this study confirmed the potential of using $A_{c}$ as scalar for the remote sensing scaling up of sap flow measurements in large, sparse vegetation areas of WLE such as Sardón catchment. In contrast, $D B H$ and $A_{s}$ are rather to be used for scaling up at stand scale, because they cannot be retrieved from remotely-sensed images.

\subsubsection{Up-scaled transpiration and spatial patterns}

The scaled-up, canopy-normalised transpiration in the Sardón catchment for Q.i. ( $\left.T_{t-Q . i .}\right)$ was between 0.38 and $3.27 \mathrm{~mm} \mathrm{day}^{-1}$, with a mean of $0.83 \mathrm{~mm} \mathrm{day}^{-1}$ and a standard deviation (SD) of $0.77 \mathrm{~mm} \mathrm{day}^{-1}$ (for 30,671 Q.i. trees in the catchment). In the case of Q.p., the scaled-up canopy-normalised transpiration $\left(T_{t-Q . p .}\right)$ was between 0.59 and 3.60 $\mathrm{mm}$ day $^{-1}$, with a mean of $1.19 \mathrm{~mm}^{-1 a y^{-1}}$ and an SD of $0.85 \mathrm{~mm}$ day $^{-1}$ (for 153,757 Q.p. trees in the catchment). The larger $T_{t}$ of Q.p. was due to generally more significant influence in the Equation 4.8 of the higher $J_{p}$ in Q.p. as compared to the higher $A_{x} / A_{\mathcal{C}}$ ratio in Q.i..

Considering Q.p., our $T_{t-Q . p .}$ measurement-range $(0.59-3.60 \mathrm{~mm}$ day $^{-1}$ ) was similar but with a higher minimum and maximum than the ones of the study of Hernández-Santana et al. (2008a) $(0.2-3.2 \mathrm{~mm}$ day $^{-1}$ ), in a Mediterranean mountain forest approximately $200 \mathrm{~km}$ south of the Sardón catchment. The difference in $T_{t-Q . p .}$. could probably be related to different environmental conditions characterized by more humid and cooler climatic conditions with rainfall of $\sim 1000 \mathrm{~mm}_{\text {year }}{ }^{-1}$ and mean yearly temperature of $10 a^{\circ} \mathrm{C}$ s compared to $\sim 500 \mathrm{~mm}_{\text {year }}{ }^{-1}$ and $13.5 i^{\circ} \mathrm{C} \mathrm{n}$ the Sardón study area. However, it is also likely that Hernández-Santana et al. (2008a) underestimated their sap flow measurements using TDP method because the TDP does not allow to detect night flow whenever it occurs regularly at night, as it was the case of all measurements in Q.p. trees described by Reyes-Acosta and Lubczynski (2014). 

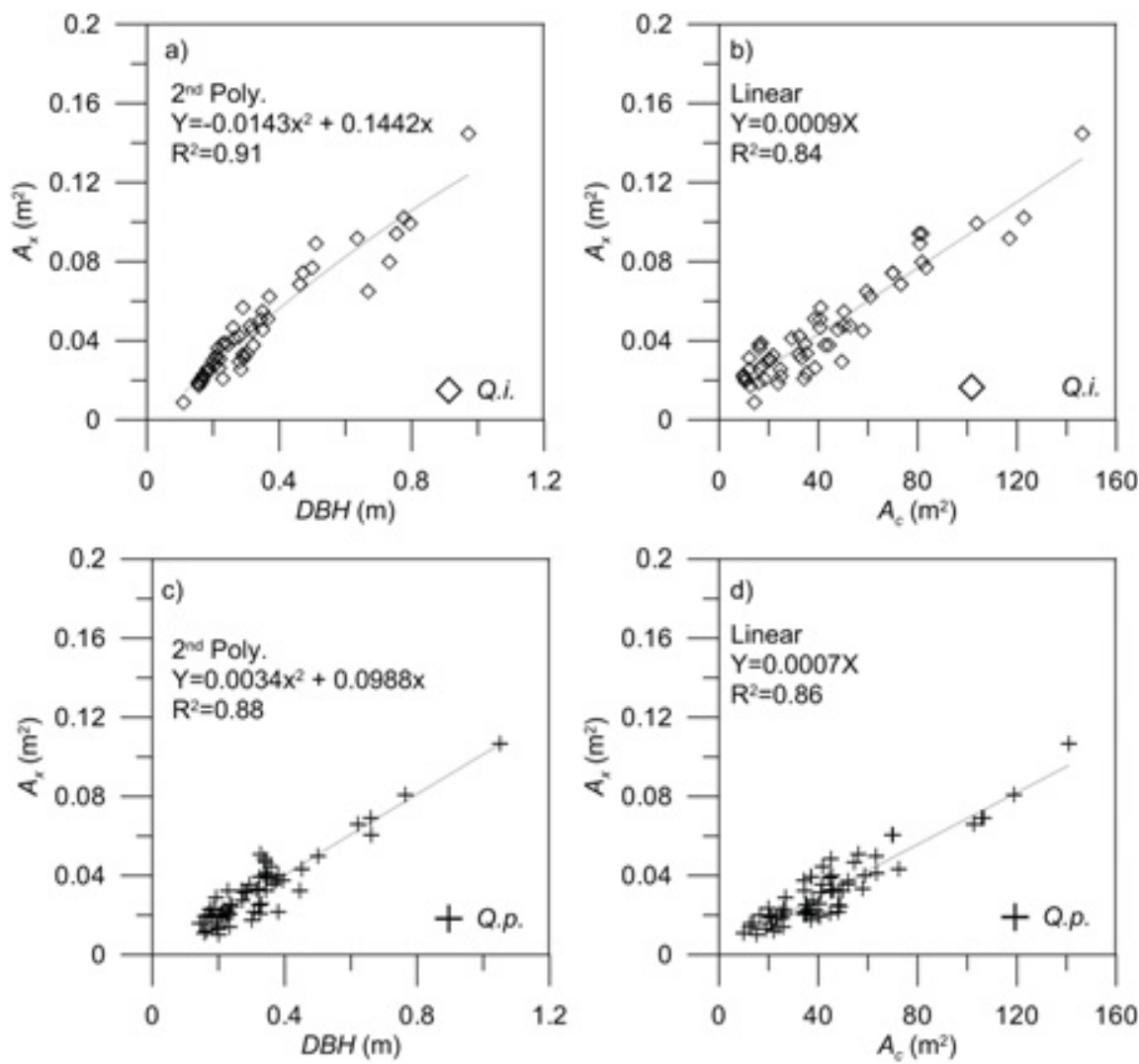

Figure 4.8: Biometric-upscaling functions (BUFs) for Q.i. and Q.p., derived from the biometric parameters collected in the tree survey. The BUFs a) and c) follow a $2^{\text {nd }}$ polynomial fitting model for both species respectively, and are useful for ground measurements upscaling. Whereas BUFs b) and d) follow a linear fitting model and are useful for RS upscaling. The respective coefficient of determination $\left(R^{2}\right)$ for each model is provided. 


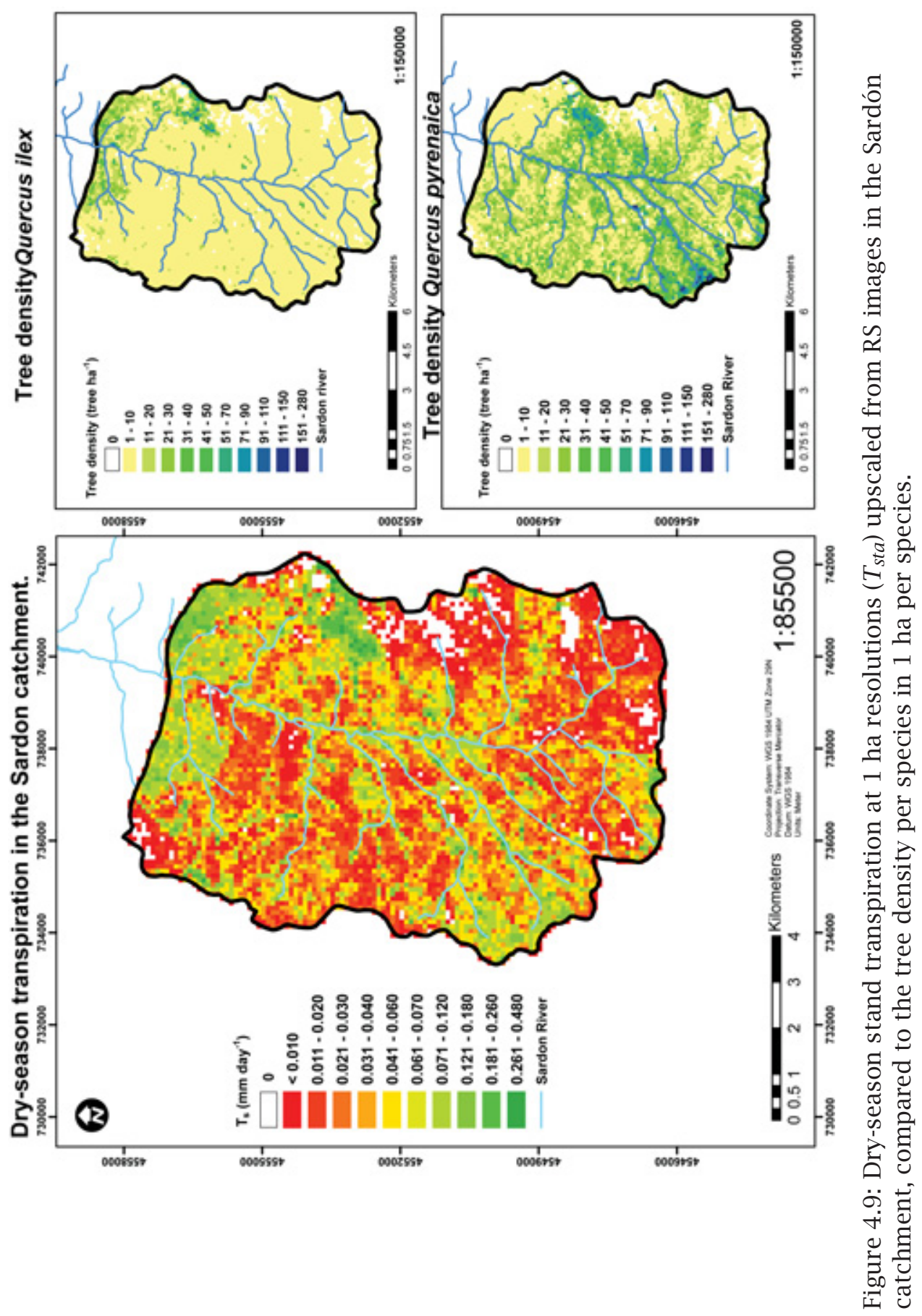


The remote-sensing-based, scaled-up dry-season tree transpiration at the 1 ha stand scale $\left(T_{\text {sta }}\right)$ ranged from 0 to $0.471 \mathrm{~mm} \mathrm{day}^{-1}$ across the catchment (Figure 4.9a); the catchment scale dry-season tree transpiration $\left(T_{c}\right)$ was $0.045 \mathrm{~mm} \mathrm{day}{ }^{-1}$ (average of $T_{\text {sta }}$ for the whole catchment) with a standard deviation of $0.041 \mathrm{~mm} \mathrm{day}{ }^{-1}$. From the obtained $T_{c}$, Q.i. and Q.p. trees contributed $21 \%$ and $79 \%$, respectively. The higher contribution of Q.p. trees to dry-season $T_{C}$ is driven mainly by the greater number of Q.p. trees per hectare (average 19 trees ha $^{-1}$ ) than Q.i. trees (average 4 trees $\mathrm{ha}^{-1}$ ). The $T_{\text {sta }}$ varied widely across the catchment as indicated in Figure 4.9 and by the SD of $T_{\mathcal{C}}\left(T_{\mathcal{C}} \approx S D\right)$. That variability was obviously proportional to tree density. The highest transpiration fluxes were primarily located closer to the stream valleys where tree density was the highest resulting in $T_{\text {sta }}$ ranging between 0.181 and $0.480 \mathrm{~mm}$ day $^{-1}$. The areas outside valleys had typically $T_{\text {sta }}$ between 0.011 and $0.040 \mathrm{~mm} \mathrm{day}^{-1}$.

Figures 4.9b and 4.9c show respectively Q.i. and Q.p. spatial variations of tree densities. Q.i. trees dominated only at the north-eastern side of the catchment, far from the streams and river valleys (Figure $4.9 \mathrm{~b}$ ). The spatial distribution of Q.i. trees suggests that this species is more abundant in locations with relatively deep water table (i.e. far from streams and valleys), because it can overcome water-stress during the dry-season due to its deep root system that is hydraulically connected to deep water sources. In such locations, Q.i. trees can maintain a conservative water-use strategy with a quasi-constant water supply from deep sources when the top soil is dry (David et al., 2007), a strategy typical for this species (Mediavilla and Escudero, 2003). In contrast, Q.p. trees were abundant in the river valleys and in the south-western part of the catchment (Figure 4.9c), i.e. in locations where soil moisture availability and/or water table was close to the surface throughout the dry season (confirmed by soil moisture profiles measured during 2009), allowing them to use a non-conservative water-use strategy and cope with the typically high transpiration demands as previously described by Hernández-Santana et al. (2008a) and Moreno et al. (2011).

Interestingly, $T_{\text {sta }}$ was the highest in the north-eastern areas, where Q.i. trees dominated (Figure 4.9a and 4.9b), even though the mean measured sap flux density $\left(J_{p}\right)$ for Q.i. trees was lower than that of Q.p. trees (Figure 4.3 and Table 4.1). This was because: the BUF used for remote-sensing-based scaling up was steeper for Q.i. trees (Figure $4.8 \mathrm{~b}$ ) than for Q.p. trees (Figure 4.8d) resulting in larger $A_{x}$ for given $A_{c}$, and also because $Q . i$. trees in that area are numerous, old and large, so their individual water uptakes are also large due to their higher $A_{x}$ areas.

A cross-reference of this study with the research of Balugani et al. (2011) on the dry season actual evapotranspiration $\left(E T_{a}\right)$ measured from an eddy-covariance flux tower carried out in the same Sardón study area was made. It showed that the dry-season tree transpiration within the footprint of the tower $\left(T_{f}=0.036 \mathrm{~mm} \mathrm{day}^{-1}\right)$, scaled up with the same BUF as in this study, was comparable with the scaled-up catchment transpiration $\left(T_{c}=0.045 \mathrm{~mm} \mathrm{day}^{-1}\right)$ but represented only $6 \%$ of $E T_{a}(0.6$ 
mm day $\left.{ }^{-1}\right)$. Such low $T_{f}$ and $T_{c}$ as compared to $E T_{a}$ was likely related to: (a) the low tree-density and vegetation cover of Q.i. and Q.p. trees (the average canopy coverage under the eddy-covariance foot print and in the catchment was $\approx 7 \%$, implying low $T_{\text {sta }}$; (b) the location of the eddy tower which was in an area where $T_{\text {sta }}$ was low due to the small size of the canopies $\left(A_{c}\right)$; and $(c)$ a shallow water-table $(5 \mathrm{~m})$ in the foot-print area, so that direct bare-soil evaporation of groundwater likely made a larger contribution to $E T_{a}$ than $T_{c}$ (Balugani et al., 2011).

The tree-population structure of the Sardón catchment, with an average tree density of $\approx 19$ trees ha ${ }^{-1}$, is lower than typical Mediterranean woodlands (30-60 trees ha ${ }^{-1}$ ) (Carreiras et al., 2006; David et al., 2004; Moreno et al., 2005), but not uncommon (Carreiras et al., 2006; Smit et al., 2008). The observed spatial patterns suggest a division of ecological niches (e.g. distribution of resources and potential competitors) between Q.i. and Q.p. species, which is likely to decrease inter-specific competition (i.e. between species) for limiting resources, in this case soil water availability. This conclusion is further supported by the fact that in the catchment both species are not found in mixed clusters and rarely close to each other. Human intervention may be another factor shaping the current structure and distribution of vegetation in the Sardón catchment. Human activities can, indeed, be considered to have an impact on the trees, since farming practices in the area are focused on protecting and facilitating conditions favourable for Q.i. trees, due to their socioeconomic role for pig-grazing under the trees (such pigs are used for the production of "Bellota iberico" ham). In contrast, Q.p. trees are relatively less-managed and have no particular economic use in the region, they are sometimes pruned for ornamental reasons or used as fuel. One last, indirect factor that has been reported to affect populations of Q.i. species is grazing intensity, which may not allow normal recruitment of seedlings (Smit et al., 2008). However, this factor has a higher effect at local scales than that of the catchment level, where water availability in dry seasons, is the most important factor explaining species distribution in semi-arid areas (Porporato et al., 2001).

\subsubsection{Comparison of remote-sensing and stand scale scaling up}

The analysed 1 ha stand contained 8 Q.i. and 5 Q.p. trees of varying sizes (See Table 4.5); all trees had been biometrically surveyed. The stand-scale scaling up of the selected area resulted in a $T_{\text {sta }}$ value of $0.047 \mathrm{~mm} \mathrm{day}^{-1}$, or $469.4 \mathrm{l} \mathrm{day}^{-1}$, while remote-sensing-based scaling up of the same stand $0.046 \mathrm{~mm} \mathrm{day}^{-1}$ or $461.4 \mathrm{l} \mathrm{day}^{-1}$ (See Table 4.5). Despite lower amount of trees in the analysed stand (13) than the catchment mean (17), the estimated with both methods $T_{\text {sta }}$ were slightly larger than the catchment mean $T_{\text {sta }}(0.04+/-0.04 \mathrm{~mm}$ day- 1$)$. This was because the trees in the selected stand were generally larger than the mean size trees in the catchment. When using the mean absolute error (MAE) and root mean squared error (RMSE) to compare both techniques, it was found that MAE was $0.83 \mathrm{l} \mathrm{day}^{-1}$ while RMSE was $1.71 \mathrm{l} \mathrm{day}^{-1}$. These results 
indicate that differences between upscaling techniques were very low $(<$ $2 \%$ ). The small differences between the two scaling up techniques can be explained by generally higher $R^{2}$ found for the $D B H-A_{x}$ BUF (applied to the stand-scale scaling up) than the $R^{2}$ for the $A_{\mathcal{c}}$ vs. $A_{x}$ BUF (applied to the remote-sensing-based scaling up), and due to the eventual errors in the delineation of the canopies from the remotely sensed images. Nevertheless, the comparison between scaling up methods indicated a strong agreement in the $T_{\text {sta }}$ calculations at the stand scale, underlining the potential of using spatial imagery of high resolution for scaling-up studies in the future.

Table 4.5: Comparison between upscaling techniques for $T_{\text {sta }}$ at 1 ha selected from the biometric ground measurements.

\begin{tabular}{ccccc}
\hline $\begin{array}{c}\text { Trees species } \\
\text { in the stand }\end{array}$ & RSUPS & FMUPS & $\begin{array}{c}\text { Absolute } \\
\text { Error } \\
(\mathrm{AE})\end{array}$ & $\begin{array}{c}\text { Squared } \\
\text { Error } \\
(\mathrm{SE})\end{array}$ \\
\hline & $\left(\mathrm{L} \mathrm{day}^{-1}\right)$ & $\left(\mathrm{L} \mathrm{day}^{-1}\right)$ & $\left(\mathrm{L} \mathrm{day}^{-1}\right)$ & $\left(\mathrm{L} \mathrm{day}^{-1}\right)$ \\
\hline Q.i. & 197.53 & 211.29 & 13.76 & 189.34 \\
Q.i. & 131.52 & 140.71 & 9.19 & 84.46 \\
Q.i. & 121.81 & 30.32 & 8.51 & 72.42 \\
Q.i. & 80.26 & 85.90 & 5.64 & 31.81 \\
Q.i. & 62.59 & 65.92 & 3.33 & 11.09 \\
Q.p. & 60.23 & 60.36 & 0.13 & 0.02 \\
Q.p. & 41.95 & 44.41 & 2.46 & 6.05 \\
Q.p. & 40.00 & 42.40 & 2.40 & 5.76 \\
Q.i. & 34.01 & 34.09 & 0.08 & 0.01 \\
Q.p. & 31.27 & 31.35 & 0.08 & 0.01 \\
Q.p. & 23.06 & 23.12 & 0.06 & 0.00 \\
Q.p. & 17.19 & 18.03 & 0.84 & 0.71 \\
Q.i. & 5.075 & 5.08 & 0.01 & 0.00 \\
\hline $\begin{array}{c}\text { Total stand } \\
\left.\text { (L day }{ }^{-1}\right)\end{array}$ & 846.49 & 892.98 & & \\
\hline $\begin{array}{c}\text { Total Stand } \\
\text { (mm day }\end{array}$ ) & 0.085 & 0.0089 & & \\
\hline $\begin{array}{c}\text { Mean AE } \\
\text { Root Mean SE }\end{array}$ & & & & \\
\hline & & & \\
\hline
\end{tabular}

\subsubsection{Uncertainty analysis of remote-sensing-based scaling up}

It is important to first clarify that species-specific $T_{c}$ referred to in this section $\left(T_{\mathcal{C S}}\right)$ is an abstract term that does not have hydrological meaning, 
but it is used to represent transpiration contributions of each species spatially. The species-specific $T_{c S}$ was calculated by dividing the sum of all tree sap flows of the particular tree species by the stand area, and then by averaging the values across the 1 ha-resolution grid for the whole catchment.

The assessment of the uncertainty after 1000 Monte Carlo iterations showed that the combined effect of the species classification errors and the errors in the BUFs resulted in an average $T_{c s}$ of $0.012 \mathrm{~mm} \mathrm{day}^{-1}(S D$ $<0.001 \mathrm{~mm} \mathrm{day}^{-1}$ ) for Q.i. trees and an average $T_{C S}$ of $0.032 \mathrm{~mm} \mathrm{day}^{-1}$ $\left(S D<0.001 \mathrm{~mm} \mathrm{day}^{-1}\right)$ for Q.p. trees (Figures 4.10a and 4.10b). In the case of $T_{C}$ for all trees in the catchment, it was in average $0.044 \mathrm{~mm}$ day $^{-1}\left(S D<0.001 \mathrm{~mm} \mathrm{day}^{-1}\right)$ (Figure 4.10c). The average $T_{C S}$ for $Q . i$. and for $Q . p$. trees were not significantly different as compared with the $T_{\mathcal{C}}$, for which the error was considered negligible due to the optimisation routine used for reducing $J_{p}$ measuring errors in the field.
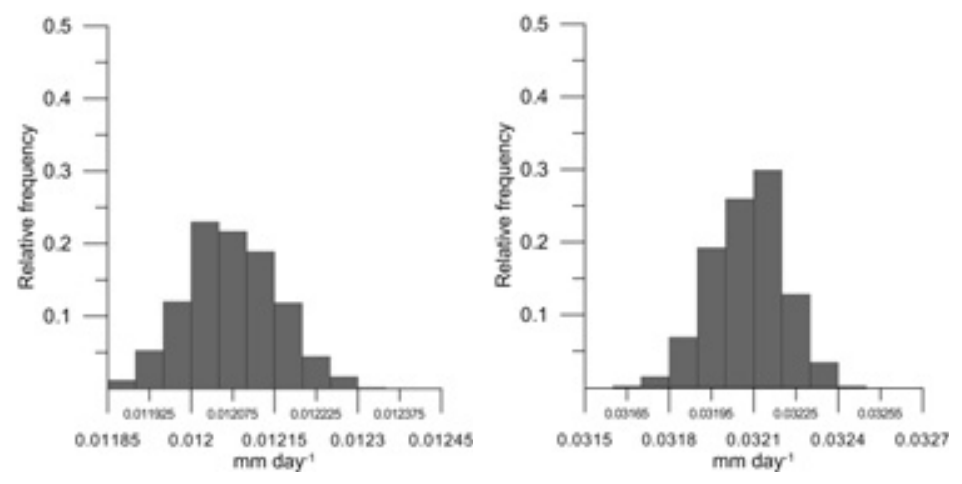

a

b

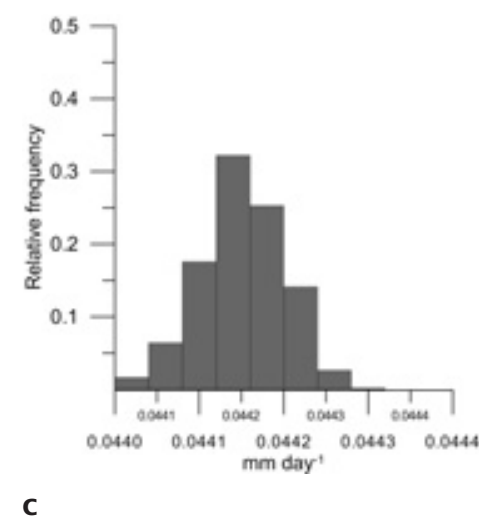

Figure 4.10: Relative frequency distribution of the transpiration at the catchment level $\left(T_{C}\right)$ obtained from Monte-carlo simulations (1000 iterations) of the RUS technique, for (a) Q.i., (b) Q.p. and (c) for both species (all trees in the catchment). 
Two factors explain the non-significant difference between the $T_{C S}$ values of the uncertainty analysis and the initial estimations of $T_{c}$. First, the "User's" accuracy of the classification map indicated that at least $12 \%$ of Q.p. trees were in fact Q.i. trees, thus, during the Monte-Carlo simulations, $\approx 18000$ trees (out of 153757 ) were added to the $T_{c S}$ calculation for Q.i. trees. Second, at least $4 \%$ of the Q.i. trees were in fact Q.p. trees, thus $\approx 1000$ trees (out of 30671 ) were added to the $T_{\mathcal{C S}}$ calculation for Q.i. trees.

The combination of both factors created a compensation effect that was observed during the simulations. When the number of $Q . p$. trees was reduced, the number of $Q . i$. trees increased, thus increasing $T_{\mathcal{C}}$, and vice versa. Therefore, as the error in the $Q . p$. classification was higher than for the Q.i. classification, and because $Q . p$. had more trees in the catchment, the average $T_{c}$ obtained from the uncertainty analysis (for 1000 iteration) was slightly, but not significantly, lower than the $T_{c}$ without any accounting for uncertainty (fewer Q.p. trees lead to a lower $T_{\mathcal{C}}$.

The $T_{C}$ simulations converged to specific values (Figure 4.10), which is also confirmed by their low $S D$, thus indicating that the combination of errors in the classification map and the BUFs had only a slight effect on the estimation of transpiration $(<1 \%)$. This demonstrates that the assessed uncertainty for the remote-sensing-based scaling up was not significant, confirming the robustness of the estimations for sparse oak-woodlands of the the Sardón catchment.

It is important to note that the the proposed method was applied to dry season-tree transpiration assessment, but if there is appropriate sap flow data available, it can be used for wet conditions with comparable uncertainties. As the accuracy of the proposed scaling-up procedure does not depend on the season assessed, but on the accuracy of sap flow measurements together with the accuracy of the scaling-up procedure, no difference of uncertainty across seasons is expected.

\subsection{Conclusions}

In this study we proposed a method for mapping dry-season tree transpiration by scaling up sap flow measurements using object attributes identified on high-resolution remotely-sensed satellite images. The method here described is novel and more advanced than previous applications because of the following: (1) we introduced in the scaling-up protocol radial and azimuthal sap flow measurements; (2) we assigned sap flow magnitudes of individual trees by categorizing sap-flux densities with respect to the sapwood area, thus enhancing the accuracy of the scaled up estimation; (3) in the scaling up procedure, instead of considering whole sapwood areas for sap-flow calculations, we considered flows of individual sapwood annuli to enhance the precision of the estimations; (4) for defining the attributes of the vegetation spatially in the scaling up procedure, we used a combination of two high resolution multis- 
pectral images (QuickBirs and World View II) acquired in two different growing seasons; and (5) finally we provided a Monte Carlo uncertainty estimations of the procedure.

Through the scaling up of transpiration and the artificial neuralnetworks (ANN) simulations we discovered that the tree transpiration in the studied oak woodland ("dehesa") was low $\sim 0.04 \mathrm{~mm}$ day ${ }^{-1}(+/-0.04)$ and changed minimally throughout the dry season. Furthermore, we found large spatial variability of tree transpiration ( $T_{\text {sta }}$ spatial patterns), mainly determined by the density of the Q.i. and Q.p. tree species constrained by the water availability either as soil moisture or shallow groundwater. This spatial variability information bears an important potential to understand the eco-hydrological role of the Q.i. and Q.p. species and to promote adequate water management practices in semiarid areas, such as as the Sardón catchment.

Transpiration mapping by remote sensing scaling up of sap flow measurements is a convenient tool for understanding the hydrological dynamics of the plant-soil-water interactions but also for quantitative estimates of plant water uptake in future studies. 



\section{Sourcing dry-season tree transpiration}

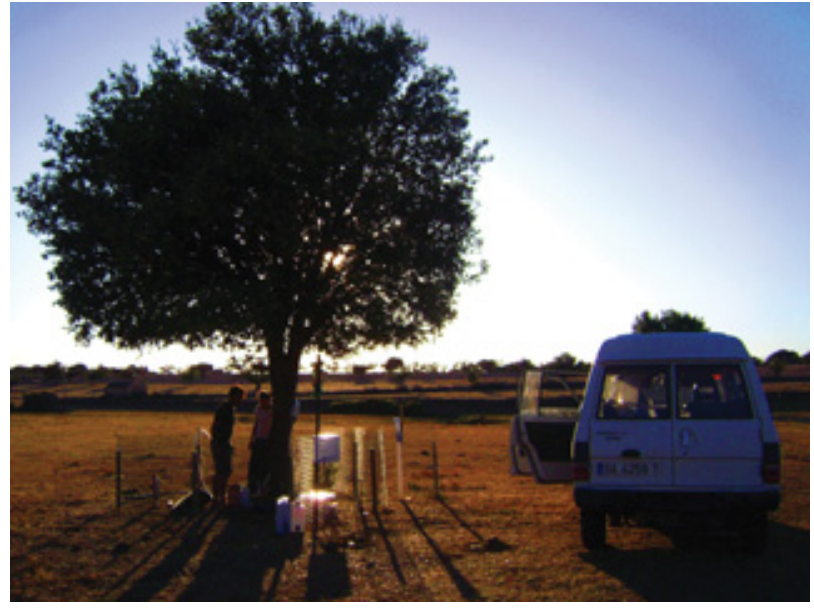

Figure 5.1: Deuterium tracing experiments designed to distinguish water sources of transpiration in a Quercus ilex tree at the end of the dry season 2009 (August). 


\begin{abstract}
Studies have attempted to disaggregate the sources of water that contribute to the transpiration in trees, but there is little knowledge about the driving forces causing certain sources to be more intensively used than others. To understand the dynamics of water abstraction from Quercus ilex (Q.i.) and Quercus pyrenaica (Q.p.) during the dry season, this study combined the information obtained from tracing deuterium (a naturally abundant isotope denoted as $\delta^{2} H$ ) to obtain water contributions, with modelling whole-tree (WT) water flows to explain these contributions. The isotopic measurements indicated that Q.i. and Q.p. trees abstracted $>90 \%$ of the water used for transpiration from the saturated zone in the beginning of the dry season, whereas at the end of the dry season, this contribution was reduced to $\approx 40 \%$. The calibrated WT model provided temporally variable water uptakes from the saturated and unsaturated zones and the distribution of water potentials throughout the tree. By combining the $\delta^{2} H$ tracing results with the WT model, it was possible to replicate and better understand the physiological mechanisms controlling the water potential forces to abstract water from different soil compartments. It was found that water availability in the subsurface in the beginning and more humid period of the dry season (WET) facilitated abstraction from subsurface layers (75 to $100 \mathrm{~cm}$ deep) hydrologically connected to the water table. In contrast in the end and driest period of the dry season (DRY), the water availability in the soil decreased dramatically and the groundwater was deeper and thus less easy to to abstract (>150 to $300 \mathrm{~cm}$ ). Such dry conditions triggered higher dependency on the water available in shallow soil layers (25 to 75 cm deep); a decrease in groundwater abstraction; and therefore a higher use of stored water indicated by larger variations in the expansion and contractions of the stem diameter. Combining isotopic tracing measurements and a modelling framework revealed that (a) the water contributions of groundwater and unsaturated layers to Q.i. and Q.p. transpiration are determined by water table depth during the WET period; whereas (b) moisture conditions in the soil during the DRY period determined the water contributions from the unsaturated layer and the groundwater; and that (c) water storage plays a crucial role in maintaining transpiration rates during the peak of the dry season, and a significant amount of that stored water originates from groundwater.
\end{abstract}

\title{
5.1 Introduction
}

Trees are important components for the regulation of the hydrological dynamics of ecosystems. They uptake water from different hydrological compartments, such as moisture from the soil, groundwater or even from mist, depending on climatic conditions (Burgess and Dawson, 2004; Lubczynski, 2009; Nadezhdina et al., 2010). Understanding and quantifying the dynamics behind such water uptakes is key knowledge for a proper environmental management, especially for those environments 
more sensitive to climate change, such as water limited environments (WLE) (Newman et al., 2006). Several studies have attempted to establish the contribution of different soil compartments to the transpired water using stable isotopes (Thorburn et al., 1993; Dawson et al., 2002; Zencich et al., 2002; Phillips and Gregg, 2003; David et al., 2007). These studies have been successful in separating the volumetric water contributions between groundwater and soil moisture of the unsaturated zone, especially during drier conditions (Zencich et al., 2002; Phillips and Gregg, 2003). However, there is little knowledge on the mechanisms controlling the spatio-temporal variability of such contributions (i.e. soil moisture, air humidity, plant architecture, water potentials, etc.).

The mechanisms determining the sources of transpiration, referred to as sourcing, can be of a different nature, namely: biological, ecological, hydrological, etc. However, when following Liebig's law of the minimum (Reilly and Fuglie, 1998) the plant growth is controlled not by the total amount of resources available, but by the scarcest resource. It is most probable that when water availability is the limiting factor, the abstracted water originates from the highest water potentials (i.e. potential energy) in the subsurface at a specific time and place. The differences in water potential determine the flow of water in the soil-plant-atmosphere continuum (Edwards et al., 1986; Tyree, 1988; Tyree et al., 1991; Tyree and Zimmerman, 2002; Steppe et al., 2006), and thus would determine the movement of water in roots from and throughout different compartments. Soil in this study is understood as subsurface material (not as a specific zone) and may be present in the unsaturated and saturated zones.

The movement of water driven by water potential difference is an intricate phenomenon because it depends on the in-situ characteristics of trees, generally: structure of live canopy, amount of xylem in the trunk and its density, root density, root depth, soil moisture profile, soil salinity, solar radiation and vapour pressure deficit (Tyree et al., 1991; Steppe et al., 2006; Wheeler and Stroock, 2008). Thus, to explain and quantify the specific source contributions of different subsurface water compartments for a tree, it is ideal to: (1) simultaneously monitor the water potential in all the compartments, (2) trace water flow in different tree compartments and subsurface water compartments, and (3) measure transpiration rates (e.g. using sap flow measurements).

Such a measuring design is feasible but very complex and equipment demanding, therefore it is of limited applicability especially at high spatial/temporal scales. An alternative is to use a modelling technique that integrates the principles of the water movement in plants with sufficient level of detail to determine the changes in water uptake as a function of plant resistances and water availability. Such a modelling approach can provide a hydrological explanation (e.g. linkage between water potentials, resistances, storage, etc.) of the proportion of abstracted water sources from different water-compartments, and thus further explain the volumetric water-proportions estimated from the isotope tracing sourcing. 
Early modelling efforts during the 80s approached the hydrological behaviour of a tree in response to a water potential gradient using fundamental hydraulic theories and laws of physics (Edwards et al., 1986), tied to Tyree's cohesion-tension theory (see Tyree, 1988). Later in that decade, throughout the 90s and early in the 2000s, it was proposed to take into consideration the hydraulic architecture of trees (Tyree et al., 1991; Tyree and Zimmerman, 2002) to add accuracy to the water dynamic estimates. However, considering this complex architecture inevitably made the mathematical discretisation of the models too complicated to have wider applications.

New findings in the early 2000s concerning tree physiological processes such as hydraulic redistribution (Zweifel et al., 2000; Brooks et al., 2002) and water storage provided more evidence of the sophistication of water flows in trees. These findings sparkled interest in producing new modelling approaches to understand further the movement of water in trees. Moreover, significant improvements in computing power and portability facilitated the automation of complex modelling tasks. For example, Steppe et al. (2006) proposed a stem diameter model coupled to a single-tree flow model to simulate stem diameter variation resulting from changes in water storage and growth, while Amenu and Kumar (2007) proposed a stochastic approach to simulate the hydraulic redistribution. These approaches can explain the source contributions to transpiration temporarily. The approach of Steppe et al. (2006) is of particular interest due to its simplicity and consideration of water storage, a critical mechanism to include for an accurate quantification of water use in trees.

Despite such advantages, there is not (to the author's knowledge) a unified model that can simulate temporal dynamics of water sourcing from all hydrological compartments of the atmosphere-tree-soil continuum, including the saturated zone, while considering plant physiological mechanisms such as stem diameter fluctuations (Steppe et al., 2006) and/or hydraulic redistribution (Burgess and Bleby, 2006). This, together with the scarcity of experiments to validate initial findings of water abstractions, highlights the need for an explanation of the factors determining the way trees abstract water and the impact on higher organisational scales (i.e. catchments, forests, etc.).

Consequently, the objectives of this study were: (a) to determine contributions of the groundwater and the unsaturated zone water sources to the transpiration of Q.i. and Q.p. trees at different soil moisture levels, and (b) to adapt the single-tree sap flow model of Steppe et al. (2006) in order to quantify, validate and further understand the dynamics of these contributions. 


\subsection{Materials and methods}

\subsubsection{Sourcing of tree transpiration by tracing naturally abundant isotopes}

In this study we used stable isotope tracers for sourcing water contributions to tree transpiration $(T)$, originating either from the saturated zone $\left(T_{g}\right)$ and/or from the unsaturated zone $\left(T_{u}\right)$. This method relies on measuring the composition of naturally abundant isotopes in water to determine isotopic water contributions of different hydrological sources (Dawson et al., 2002). The isotope tracing was performed on one Q.i. and one Q.p. tree from $23^{r d}$ August until $7^{\text {th }}$ September in 2009; and the same trees again from $30^{\text {th }}$ May until $5^{\text {th }}$ June in 2010. Trees were selected according to the following criteria: no apparent sign of decay or illness; close to the automatic data acquisition stations; far from outcrops, in order to have a well-developed subsurface (deeper than 1 $\mathrm{m}$ ); protected from cattle and accessible. See Figure 5.2 for the exact location of the trees within the catchment.

Meteorological conditions during each sampling periods were fairly similar (see Table 5.1), and the most significant difference between them was in water table depth (see Subsection 5.3.1) and soil moisture (see Subsection 5.3.1), as will be discussed in the rest of the chapter.

Table 5.1: Average meteorological conditions during isotopic sampling, from an automatic data acquisition station in the proximity of the sampled trees. $\mathrm{RH}=$ relative humidity, $\mathrm{VPD}=$ vapour pressure deficit, $\mathrm{iSR}=$ incoming solar radiation and Ws $=$ wind speed.

\begin{tabular}{|c|c|c|c|c|c|c|c|c|c|c|}
\hline & \multicolumn{5}{|c|}{$23^{r d}$ Aug. until $7^{\text {th }}$ Sep. in 2009 (DRY) } & \multicolumn{5}{|c|}{$30^{\text {th }}$ May until $5^{\text {th }}$ Jun. in 2010 (WET } \\
\hline & Air Temp & RH & VPD & iSR & Ws & Air Temp & RH & VPD & iSR & Ws \\
\hline Units & ${ }^{\circ} \mathrm{C}$ & $\%$ & Kpa & Watt $/ m^{2}$ & $\mathrm{~m} / \mathrm{s}$ & ${ }^{\circ} \mathrm{C}$ & $\%$ & Kpa & $W / m^{2}$ & $m / s$ \\
\hline Mean & 21 & 70 & 0.61 & 272 & 1.6 & 15 & 55 & 0.52 & 98 & 1.6 \\
\hline Max & 35 & 100 & 2.44 & 910 & 4.2 & 26 & 100 & 1.52 & 945 & 6.5 \\
\hline Min & 7 & 24 & 0.00 & 0 & 0.0 & 7 & 19 & 0.04 & 0 & 0.0 \\
\hline Stdev & 7 & 26 & 0.67 & 330 & 1.1 & 4 & 22 & 0.36 & 170 & 1.1 \\
\hline
\end{tabular}

\subsubsection{Sample collection and isotopic content determination}

The deuterium isotopic composition $\left(\delta^{2} H\right)$ of the sources (i.e. groundwater, soil and xylem) were determined during the dry season in two different years: first from $23^{r d}$ of August until $7^{\text {th }}$ September 2009 and then from $30^{\text {th }}$ May until $5^{\text {th }}$ of June 2010.

The 2009 campaign corresponds to the driest period of the dry season in the Sardón catchment, and is referred to as DRY in the rest of the chapter. The second campaign in 2010 corresponds to the wetter period of the dry season and is referred to as WET in the rest of the chapter. The timing of the campaigns was selected to determine the difference in 
water abstraction dynamics of one Q.i. and one Q.p. tree (see Figure 5.2) under comparable clear-sky climatic conditions (see Table 5.1).

Measurements in both periods were taken using the same sampling schemes and in general they took six to seven days per tree. During the first three days, sap flow sensors were installed on the selected trees for isotope determination, following the sap flow measuring scheme described in Chapter 3. Then, in the next two to three days, piezometers were installed to take groundwater samples and measure water table depth. The piezometers were placed radially around the trees in four main azimuthal directions ( $\mathrm{N}, \mathrm{S}, \mathrm{W}, \mathrm{E})$ at $2 \mathrm{~m}$ distance from the trees. Finally, on the last day (provided that it was not cloudy), the sampling for the isotopic determination was carried on.

Groundwater samples were taken from four piezometers and kept in hermetic conditions to avoid evaporation. Soil samples were taken in the main azimuthal directions with the help of a manual Edelman auger soil sampler (Eijkelkamp Agrisearch Equipment) at $25 \mathrm{~cm}, 50 \mathrm{~cm}, 75 \mathrm{~cm}$ and $100 \mathrm{~cm}$ under sun cover, sealed, and then immediately stored in a cool box to avoid evaporation. The soil samples were further processed to extract water by azeotropic distillation (Walker and Richardson, 1991). Soil samples were taken as reference once only because hourly sampling would have been too expensive. As moisture conditions in the soil change slowly throughout the dry season due to the lack of rain, this would incur in a small error margin. Xylem water extraction was performed directly in the field. Sapwood samples were carved out from tree trunks with a manual wood corer and then a hydraulic pump was used to extract sap water as described by Lambs and Berthelot (2002). Ground, soil and sap water isotopic ratios were analysed using the equilibration technique (Coplen et al., 1991; Brigati et al., 2002) in a specialised laboratory (IsoAnalytical Ltd.).

Tracing $\delta^{2} H$ readings with a temporal resolution high enough to distinguish dynamics throughout the day required a high number of samples. After several tests carried out on pre-sampling campaigns in 2008 (data not shown), it was decided that a 3-hourly sampling intervals were optimal in a 24-hour window, thus a total of 16 samples per investigated tree site, eight from the sapwood and eight from the groundwater (see Table 5.1 for exact dates).

The results of the sampling are expressed as the hydrogen isotope ratio $\left(\delta^{2} H\right)$ in parts per thousand (\%o) relative to the accepted standard (V-SMOW), as follows:

$$
\delta^{2} H=\left[R_{\text {sample }} / R_{\mathrm{v} \text {-smow }}\right]-1.00 \%
$$

where $R$ is the mass ratio of heavy to light isotope, in the case of this study deuterium/hydrogen. 


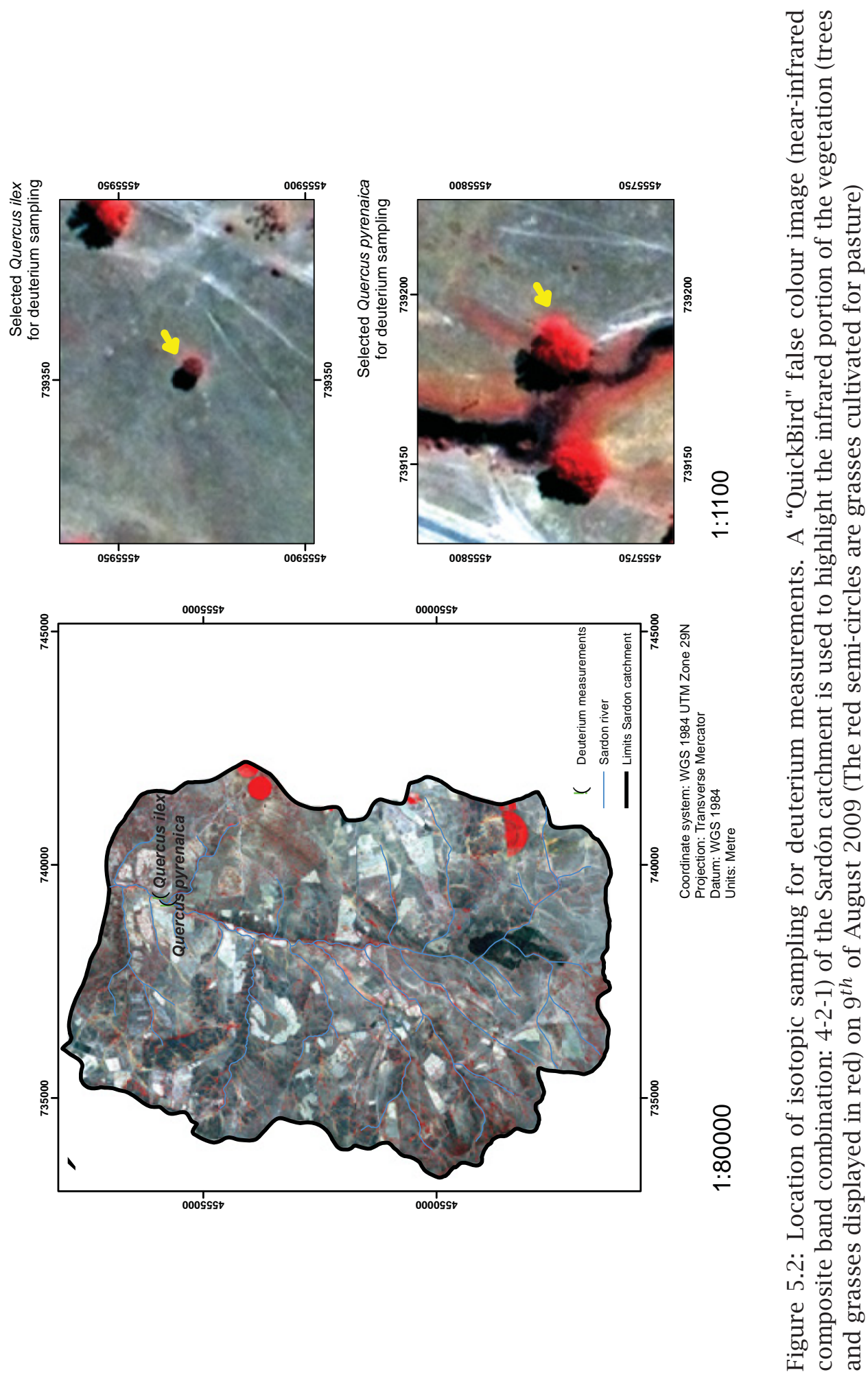




\subsubsection{Mixing models to calculate proportional contributions}

There are diverse approaches to calculating water source contributions based on isotopic compositions (Dawson et al., 2002; Zencich et al., 2002; Lubczynski, 2009). These techniques have in common that they all begin by defining a system with possible sources (e.g. water, air vapour, etc.) and a compartment where the sources mix (e.g sap water inside the tree). In this study 6 compartments were considered for both Q.i. and Q.p., as follows: 4 subsurface sources at different depths $(25 \mathrm{~cm}, 50 \mathrm{~cm}, 75 \mathrm{~cm}$ and $100 \mathrm{~cm}$ ), 1 groundwater source and $1 \mathrm{mix}$ (sap water). This set-up was selected to account for different levels of moisture in the subsurface and thus promote a clearer signal to define the contributing proportions of each source to the mix. An exception to this arrangement was used for Q.p. in the WET period only (early dry-season), when the water table was higher, thus the contributions from groundwater and former unsaturated of layer $100 \mathrm{~cm}$ deep were combined for this species.

Following Lubczynski (2009), the mixing-model to define water sources contributions to transpiration in this study were formulated as follows:

$$
\begin{array}{r}
\delta^{2} H_{t} T_{t}=\delta^{2} H_{g} T_{g} \\
+\delta^{2} H_{25 \mathrm{~cm}} T_{25 \mathrm{~cm}} \\
+\delta^{2} H_{50 \mathrm{~cm}} T_{25 \mathrm{~cm}}+\delta^{2} H_{75 \mathrm{~cm}} T_{75 \mathrm{~cm}} \\
+\delta^{2} H_{100 \mathrm{~cm}} T_{100 \mathrm{~cm}}
\end{array}
$$

where $\delta^{2} H$ is the isotopic ratio (concentration) measured in specific compartments ( $t=$ tree, $g=$ groundwater and $25 \mathrm{~cm}$ to $100 \mathrm{~cm}$ unsaturated zone compartments); $T_{t}$ is whole tree transpiration; $T_{g}$ groundwater uptake by roots and $T_{25 \mathrm{~cm}-100 \mathrm{~cm}}$ is the moisture uptake by roots from $25 \mathrm{~cm}$ to $100 \mathrm{~cm}$ unsaturated zone compartments every $25 \mathrm{~cm}$ (i.e. 4 in total).

This model describes a situation in which there are more than two sources and one isotope $\left(\delta^{2} H\right)$, hence and a unique solution cannot be found numerically only (Zencich et al., 2002). Thus, as the volumetric components and isotopic compositions of the 5 components contribute to the mixed component (sap water) only ratios $(f)$ between the mixed volume and sources are necessary to quantify the volumetric contributions (Zencich et al., 2002). In this study two approaches were used to calculate these contributions, namely: the proportional increment of Phillips and Gregg (2003), and the Bayesian approach of Moore et al. (2008). These techniques were selected due to their simplicity, sound statistical approach and successful application in determining water sources. The aim of both techniques is to use a set of ratios to satisfy the following set of mass balance equations, as described by Dawson et al. (2002):

$$
\begin{array}{r}
\delta_{\mathrm{M}}=f_{\mathrm{A}} \delta_{\mathrm{A}}+f_{\mathrm{B}} \delta_{\mathrm{B}} \cdots \\
1=f_{\mathrm{A}}+f_{\mathrm{B}} \cdots
\end{array}
$$


where $f_{\mathrm{A}}$ and $f_{\mathrm{B}}$ are ratios of the isotopic composition of the sources $\mathrm{A}$ and B to the mixture M respectively $\left(\delta_{\mathrm{M}}\right)$; and $\delta_{\mathrm{A}}$ and $\delta_{\mathrm{B}}$ are the isotopic signatures of sources A and B.

The mass balance equation for this study was derived from Eq. 5.2 and Eq.5.3, in order to apply the approaches proposed by Phillips and Gregg (2003) and Moore et al. (2008) for both Q.i. and Q.p., as follows:

$$
\begin{array}{r}
\delta_{\mathrm{t}}=f_{\mathrm{g}} \delta_{\mathrm{g}}+f_{25 \mathrm{~cm}} \delta_{25 \mathrm{~cm}}+ \\
f_{50 \mathrm{~cm}} \delta_{50 \mathrm{~cm}}+f_{75 \mathrm{~cm}} \delta_{75 \mathrm{~cm}}+ \\
f_{100} \delta_{100} \\
1=f_{\mathrm{g}}+f_{25 \mathrm{~cm}}+ \\
f_{50 \mathrm{~cm}}+f_{75 \mathrm{~cm}}+ \\
f_{100 \mathrm{~cm}}
\end{array}
$$

where $\delta_{\mathrm{t}}$ is the mixed signature in the xylem (sap water) and the five source signatures were: soil water extractions at $25 \mathrm{~cm}\left(\delta_{25} \mathrm{~cm}\right), 50 \mathrm{~cm}$ $\left(\delta_{50 \mathrm{~cm}}\right), 75 \mathrm{~cm}\left(\delta_{75 \mathrm{~cm}}\right)$ and $100 \mathrm{~cm}\left(\delta_{100 \mathrm{~cm}}\right)$ depth, and at groundwater $\left(\delta_{\mathrm{gw}}\right)$. The isotopic ratios of all members $(f)$ are defined as for Eq. 5.3.

In the case of Phillips and Gregg (2003), they formalised a general procedure to be used for any number of sources and isotope systems. The procedure first iterates each possible combination of source proportions by small increments ( $1 \%$ to $2 \%$ ), and then predicts isotope signatures for the mixture as each combination is created, satisfying the mass balance law. The predicted mixture signatures for all combinations (calculated from Eq. 5.4) are then compared with the observed mixture signatures $\left(\delta_{\mathrm{t}}\right)$. If they are equal (within a small tolerance of $1 \%$, referred to as "mass balance tolerance"), they are considered as a feasible solution and stored. The final output is a range of all possible feasible distributions (minimum and maximum). The calculations necessary to apply the technique of Phillips and Gregg (2003) in this study were processed using the IsoSource software programmed in Visual Basic by the authors.

Moore et al. (2008), proposed a stable isotope mixing model using a Bayesian framework to determine the probability distribution for the proportional contribution $\left(f_{i}\right)$ of each source $i$ to a mixture of interest, called MixSIR. They followed the same mass balance approach as in Eq. 5.3 of Phillips and Gregg (2003), although their approach has the advantage that prior information can be included to guide model estimates. The prior information must be expressed as beta-distributed curves (i.e. probabilistic distribution) and evaluated against the probabilities of source contributions. MixSIR was scripted in MATLAB, and thus the isotopic analysis of this study was executed using MATLAB scripts too. The data inputs used in this study were the averages and standard deviations of the source and mixture isotope signatures and of the fractionation values. The fractionation values for $D_{2} O$ were taken from Ellsworth and Williams (2007), and the informative priors were specified by the $\alpha$ and $\beta$ parameters defining the expected source contribution distributions. For 
this study the priors were set in all cases to indicate higher contributions from the groundwater than from the unsaturated zone water, based on studies found in the literature under comparable conditions (Zencich et al., 2002; Phillips and Gregg, 2003; David et al., 2007)

The obtained results from both techniques were compared to determine the one that provided the most constrained range of feasible source contributions to allow a unique solution, as discussed by Dawson et al. (2002). It is important to note that this chapter focuses only on the proportional water-source contributions from each source. In Chapter 6 such proportions are used to calculate water flows originated from these sources (i.e. transpiration from groundwater or the unsaturated zone) using sap flow measurements, and then scaled up to the catchment level.

Isotope tracing provides a clear indication of how water sources are abstracted, however it does not explain the tree dynamics or the mechanisms governing the specific proportions of water contribution of each water source. Therefore, the next section will describe a modelling approach selected to validate and further understand the water contributions obtained from the mixing models.

\subsubsection{Whole-tree water transport model}

The whole-tree water transport model upgraded in this study was originally developed by Steppe et al. (2006) and validated by Steppe et al. (2008) for one root-layer compartment. Steppe et al. (2006) tested two approaches to explain the dynamics of water transport, namely: a hydraulic approach and an electrical approach. Both approaches provided satisfactory results during validations but the latter was simpler and yielded better predictions, as evaluated by Steppe et al. (2006) using an objective model selection criterion. Therefore, the electrical approach was selected for adapting in this study.

The original whole-tree model by Steppe et al. (2006) did not include the saturated zone, so it could not discriminate between the water sources of the unsaturated and saturated zones. Hence, for this study the whole-tree water model was expanded, calibrated and adjusted to simulate the water flow contribution from unsaturated and saturated zones.

The expanded whole-tree water transport model of this study is composed of two modules. The first one calculates the water transport from the roots to the canopy, and the second one calculates the daily changes in stem water storage. The second module is a key component because the use of storage water is an important mechanism to reduce water deficit for tree species under dry conditions (Scholz et al., 2008).

Both modules are interconnected using a set of specific parameters.

The flow and storage modules of Steppe et al. (2006) (Figure 5.3) consists of two types of elements: storage compartments (i.e. pools) and flow path sections (Steppe et al., 2006). Two internal water storage compartments were defined by Steppe et al. (2006): the crown storage pool $\left(W_{\text {crown }}\right)$, which represents mainly first-order branches without leaves 


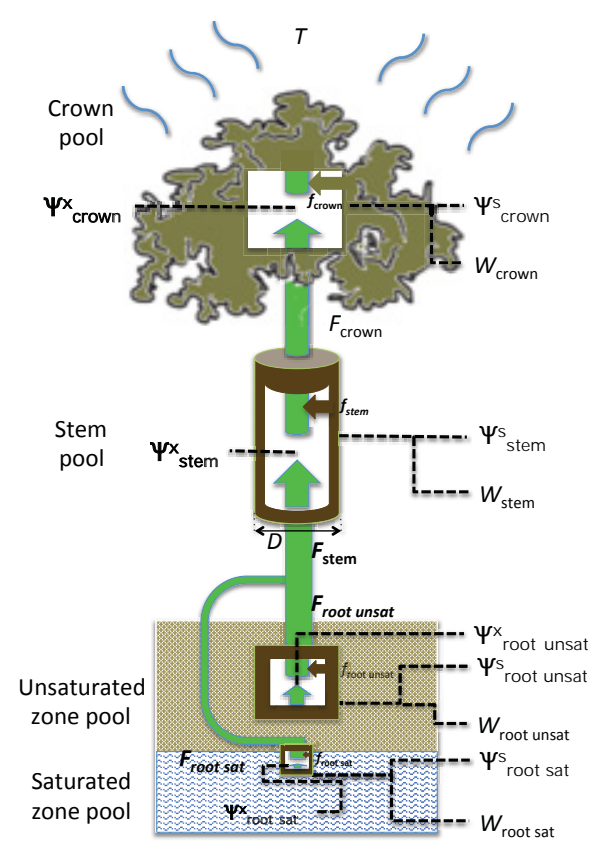

Figure 5.3: Illustration of the whole-tree water transport model including the unsaturated and saturated zones. This model consists of four compartments, interconnected by three flow paths (i.e. rates of water movement) and controlled by eight inner water potentials. The four compartments were defined as $W_{\text {crown }}=$ crown, $W_{\text {stem }}=$ stem, $W_{\text {root unsat }}$ = roots within unsaturated zone and $W_{\text {root sat }}=$ roots within saturated zone. The four flow rates were defined as $F_{\text {crown }}$ (between $W_{\text {crown }}$ and $\left.W_{\text {stem }}\right), F_{\text {stem }}$ (between $W_{\text {stem }}$ and $\left.W_{\text {root unsat }}\right), F_{\text {root unsat }}$ (between $W_{\text {stem }}$ and $W_{\text {root unsat }}$ ) and $F_{\text {root sat }}$ (between $W_{\text {stem }}$ and $W_{\text {root sat }}$ ). The water potentials in the xylem flow compartments were defined as $\Psi_{\text {crown }}^{\mathrm{x}}, \Psi_{\text {stem }}^{\mathrm{x}}$, $\Psi_{\text {root unsat }}^{\mathrm{x}}$ and $\Psi_{\text {root sat }}^{\mathrm{x}}$. The water potentials in the storage compartments were defined as $\Psi_{\text {crown }}^{\mathrm{s}}, \Psi_{\text {stem }}^{\mathrm{s}}, \Psi_{\text {root unsat }}^{\mathrm{s}}$ and $\Psi_{\text {root sat }}^{\mathrm{s}}$. Inner flows into and out of the storage compartment were defined as $f_{\text {crown }}, f_{\text {stem }}, f_{\text {root unsat }}$ and $f_{\text {root sat. }}$. And $D$ is equal to the stem diameter.

located at the top of the modelled tree, and the elastic stem storage pool $\left(W_{\text {stem }}\right)$, which includes the living part of the stem bark.

In this study the "soil" compartment defined by Steppe et al. (2006) was divided to include two storage compartments, namely: the roots in the unsaturated zone $\left(W_{\text {root unsat }}\right)$ and the roots in the saturated zone ( $\left.W_{\text {root sat }}\right)$. This modification enabled root water uptake to be differentiated between the two compartments as follows: (a) the water abstracted 
as soil moisture from the unsaturated zone, equivalent to $T_{u}$ and originating from $W_{\text {root unsat }}$; and (b) groundwater abstracted from the saturated zone and/or its hydraulically connected capillary fringe, equivalent to $T_{g}$ and originating from $W_{\text {root sat }}$. Inclusion of the capillary fringe is important, because if roots pump water from the capillary fringe this volume of water is replaced by groundwater, so the water extracted from the capillary fringe can be considered as groundwater loss. Likewise, the saturated zone is considered as an unlimited shallow water storage compartment due to the shallow water table condition in the study area (see Chapter 3). All the model compartments are interconnected and the flow between them is controlled by water potential gradients, further represented by electrical potential gradients; resistances to water flow are represented by electrical resistances and water storages by electrical capacitances.

The storage compartments are connected by four flow paths, namely (from bottom to top): $F_{\text {root unsat }}$ and $F_{\text {root sat }}$ representing separate flow paths from $W_{\text {root unsat }}$ and $W_{\text {root sat }}$ that mix into a single flow $F_{\text {stem }}$ to reach $W_{\text {stem }}$. Finally, $F_{\text {crown }}$ connects $W_{\text {crown }}$ and $W_{\text {stem }}$.

The model of interconnected pools and flow paths is driven by the transpiration rate in the top of the canopy $T$, which starts a chain of events throughout the tree (Steppe et al., 2006). First, the transpiration decreases the xylem water potential of the crown $\left(\Psi_{\text {crown }}^{\mathrm{x}}\right)$. In response to this a water potential difference develops in the xylem that induces water flow in the upper part of the tree $\left(F_{\text {crown }}\right)$. Second, as water is withdrawn from the crown storage compartment, the water content $\left(W_{\text {crown }}\right)$ decreases and the water potential of the storage $\left(\Psi_{\text {crown }}^{\mathrm{s}}\right)$ is lowered as well. The tension developed in the xylem by transpiration is transmitted to the other pools from the crown to the stem, and finally to the roots. The transmitted tension then decreases the further six inner water potentials, as follows: (i) $\Psi_{\text {stem }}^{\mathrm{x}}$ in the xylem between crown and stem, (ii) $\Psi_{\text {root unsat }}^{\mathrm{x}}$ in the xylem between stem and roots in the unsaturated zone, (iii) $\Psi_{\text {root sat }}^{\mathrm{x}}$ in the xylem between stem and roots in the saturated-zone, (iv) $\Psi_{\text {stem }}^{\mathrm{s}}$ between the stem xylem and the stem storage pool, (v) $\Psi_{\text {root unsat }}^{\mathrm{s}}$ between the unsaturated roots xylem and the storage pool, and finally (vi) $\Psi_{\text {root sat }}^{\mathrm{s}}$ between the saturated roots xylem and the storage pool.

Whereas the water potential difference in the xylem and roots induces a vertical water flow in the lower compartments of the tree $\left(F_{\text {stem }}\right.$, $F_{\text {root unsat }}$ and $\left.F_{\text {root sat }}\right)$, the water potential difference between xylem and bark causes a radial water flow from the stem storage compartment toward the xylem. As a result, the water content of the living part of the stem bark $\left(W_{\text {stem }}\right)$ decreases and, consequently, the stem diameter $(D B H)$ shrinks. The water potential of the stem storage pool $\left(\Psi_{\text {stem }}^{\mathrm{s}}\right)$ is lowered as the stem tissue contracts, and this will continue until the difference between the xylem water potential of the stem $\left(\Psi_{\text {stem }}^{\mathrm{x}}\right)$ and the storage $\left(\Psi_{\text {stem }}^{\text {s }}\right)$ is zero (Steppe et al., 2006).

Climatic data necessary to run and calibrate the model, such as VPD, relative humidity, air temperature and sap flow were collected during the field campaigns described in Chapter 3. 


\subsubsection{Water flow model assumptions}

Steppe et al. (2006) assumed that the movement of water between xylem and storage compartments in the crown and stem pools have the same exchange resistances $\left(R_{\text {crown }}^{\mathrm{s}}=R_{\mathrm{stem}}^{\mathrm{S}}=R^{\mathrm{s}}\right)$. They claim that this approximation is justified because the model aims mainly to simulate the physiological process within the tree. The same was assumed in this study for the unsaturated and saturated zone compartments $\left(R_{\text {unsat }}^{\mathrm{s}}=R_{\text {unsat }}^{\mathrm{s}}=R_{\mathrm{sat}}^{\mathrm{s}}=R^{\mathrm{s}}\right)$.

In relation to xylem resistances in $W_{\text {crown }}$ and $W_{\text {stem }}$, the assumptions of Steppe et al. (2006) were followed by setting $R_{\text {crown }}^{\mathrm{x}}$ and $R_{\mathrm{stem}}^{\mathrm{x}}$ as equal. However, in the case of the xylem resistances in $W_{\text {root unsat }}$ and $W_{\text {root sat }}\left(R_{\mathrm{unsat}}^{\mathrm{x}}\right.$ and $R_{\mathrm{sat}}^{\mathrm{x}}$ respectively), they were calibrated to reflect the proportion of shallow and deep roots in contact with moisture. Such calibration enabled the large differences in soil water potentials influencing the tree to be simulated. Finally, initial water potential of the xylem root compartments was set as equal to soil water potential (Steppe et al., 2006).

All simulations began at the end of the night, when equal values of water potential prevail in the soil-plant continuum because the transpiration is generally zero (Nobel, 1999), or significantly low (Reyes-Acosta and Lubczynski, 2014), allowing for a sufficient warming-up period before the main transpiration processes are triggered.

\subsubsection{Roots-to-canopy water transport}

The mathematical description of the water transport in the xylem flow path sections was derived from an electrical analogue of Ohm's law applied to water transport in trees (Figure 5.4). Ohm's law explains that the electric current (i.e. water flow) through a conductor (i.e. xylem) between two points is directly proportional to the electrical potential gradient (water potential gradient) difference across the two points. This means water potentials in the xylem compartments are exclusively determined by their negative pressure potential component. This is a safe assumption because the osmotic potential of the xylem sap is negligible (Jones, 1992; Nobel, 1999). This mathematical description is covered in detail in the Appendix (Subsection 5.5.1).

\subsubsection{Stem diameter variation submodel (SDVS)}

Water storage is an important mechanism to reduce water deficit for tree species under dry-water conditions, and therefore its inclusion in the model provided a more realistic simulation and understanding of the water source dynamics. Therefore, Steppe et al. (2006) designed a stem diameter variation submodel (SDVS) to simulate stem diameter variation resulting from changes in both water storage and growth. The sub-model was also included in the model for this study, and as in the version of Steppe et al. (2006) it determined the resistance of the stem storage compartment $\left(R_{\mathrm{S} \text {-stem }}\right)$. 


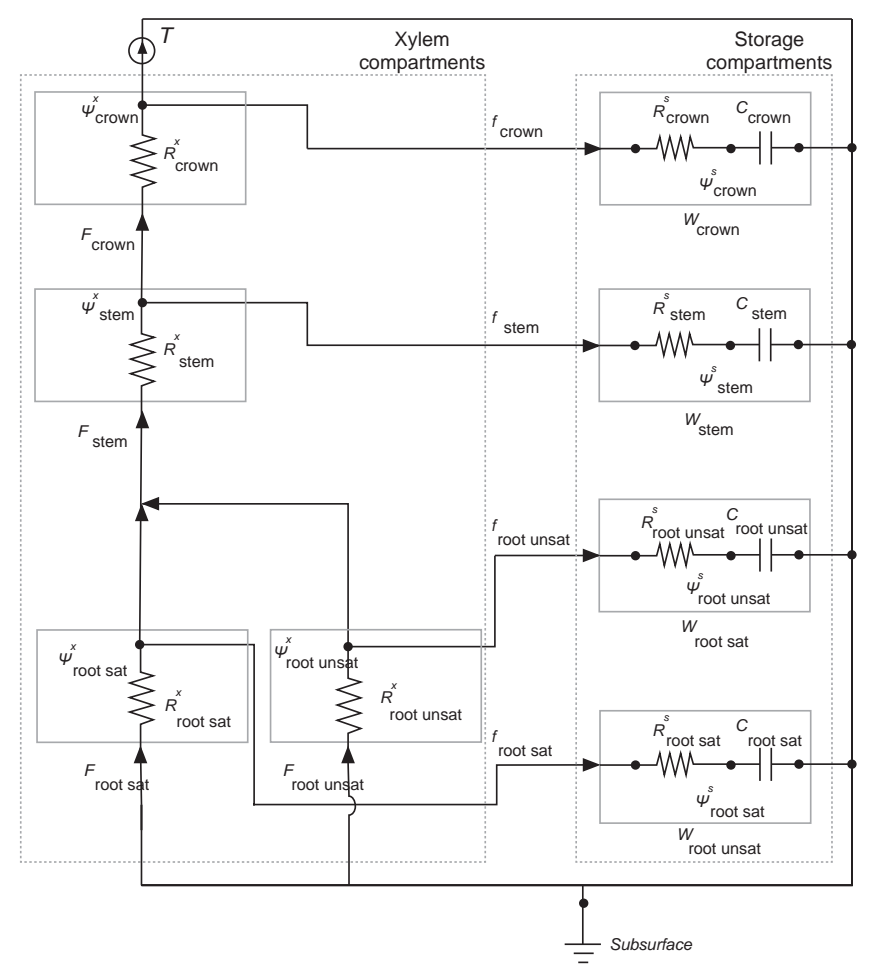

Figure 5.4: Electrical representation of the water transport model. Both the xylem and storage main compartments are grouped by dashed-line boxes. The pools are defined as crown, stem, roots in the unsaturated zone (root unsat), and roots in the saturated zone (root sat). The driving variables of the model are as follows: water content in a storage compartment $\left(W_{\text {pool }}\right)$; water potential in a storage compartment $\left(\Psi_{\text {pool }}^{\mathrm{s}}\right)$; water potential in a xylem compartment $\left(\Psi_{\text {pool }}^{\mathrm{x}}\right)$; water flow exchange between the xylem and the storage compartment $\left(f_{\text {pool }}\right)$; water flow through a xylem compartment $\left(F_{\text {pool }}\right)$; flow resistance in between a xylem and a storage compartment ( $R_{\text {pool }}^{\text {s }}$ ); flow resistance between xylem compartments $\left(R_{\text {pool-pool }}^{\mathrm{x}}\right)$; and capacitance of the storage compartment $\left(C_{\text {pool }}\right)$.

For modelling the stem diameter variation process Steppe et al. (2006) considered the stem as two coaxial cylinders separated by a "virtual" membrane, as proposed by Génard et al. (2001). The external cylinder was considered as the stem storage compartment that swells and shrinks in response to radial water flow from the xylem. The "virtual" membrane represents several cell layers containing membranes and cell walls that participate in apoplastic (movement of water and solutes through the cell walls and the intercellular spaces) and symplastic (movement of water and solutes through the continuous connection of cytoplasm) water 
flow between the overall extensive tissue and the rigid xylem system (Génard et al., 2001). For this study it was assumed that the diameter variation and water flow within the root compartments were negligible. The mathematical descriptions of the SDVS can be also found in the Appendix (Subsection 5.5.2).

\subsubsection{Model implementation}

The water flow submodel and the SDVS consist of a set of algebraic and differential equations that were solved numerically. The implementation of the equations for both modelling components was programmed and implemented using R language/platform. The whole-tree water transport model was run as the main model with a temporal resolution of one hour, and the SDVS was run as an independent module to determine the exchange resistance between the stem xylem and storage compartment for each time-step.

This modelling design was also used for the parameter optimisation sessions.

\subsubsection{Initial conditions and parameter optimisation}

The initial conditions for the model were set following Steppe et al. (2006) and Steppe and Lemeur (2007b), but the initial values for $D_{\text {outer, }}$, $D_{\text {inner }}, d^{\mathrm{s}}, \Psi_{\text {stem-p }}^{\mathrm{s}}$ and $W_{\text {stem }}$ (see Appendix 5.5) were adjusted for the average size of a Q.i. and a Q.p. tree. $D_{\text {outer }}$ was obtained from field measurements $(\approx 25 \mathrm{~cm})$. Initial values of $D_{\text {inner }}(\approx 17 \mathrm{~cm})$ and $d^{\mathrm{s}}(\approx 4 \mathrm{~cm})$ were calculated from Eqs. 5.31 and 5.33 respectively. The initial value for the pressure potential in the stem storage $\left(\Psi_{\mathrm{p}}^{\mathrm{S}}\right)$ was approximated from values observed at midnight (2.9 MPa), following the approach Génard et al. (2001) as shown by Steppe et al. (2006). The stem water potential values for Q.i. were taken from Sala and Tenhunen (1994) and Terradas and Savé (1992) (-2.16 MPa), and for Q.p. from Hernández-Santana et al. (2009) (-0.5 MPa). The initial value for $W_{\text {stem }}$ was calculated from the geometry of the stem using Eq. 5.33 and taking into account that only $40 \%$ of the total stem storage volume $V^{\mathrm{s}}$ consists of water $\left(\approx 0.056 \mathrm{~m}^{3}\right)$. The initial value of $W_{\text {crown }}$ was assumed to be $50 \%$ of the stem storage as found by Steppe et al. (2006).

The initial values for the resistances between compartments and their capacitances, in addition to the storage water content in the roots of the unsaturated and saturated zones, were optimised using the parameter optimisation algorithm "Differential Evolution Adaptive Metropolis" (DREAM) developed by Vrugt et al. (2008, 2009) (see Tables 5.4 and 5.5 for a list of optimised parameters). The values for resistance and capacitances specifically were set following Steppe et al. (2008) as initial guesses (See Table 5.2), and then optimised by increasing or decreasing the value in $1 \%$ steps, to fit the unique optimisation objective set. This was necessary because the climatic characteristics of the trees in which the models were originally developed (Fagus sylvatica L. and 
Quercus robur L. in temperate areas) are different from Q.i. and Q.p. (in Mediterranean areas).

The DREAM algorithm runs multiple different chains simultaneously for global exploration, and automatically tunes the scale and orientation of the proposed distribution using differential evolution to fit a predefined objective (Vrugt et al., 2009). This scheme is an adaptation of the SCEM-UA global optimisation algorithm (Vrugt et al., 2003b) and has the advantage of maintaining detailed balance and the same behavior averaged over time while showing excellent efficiency in complex, highly nonlinear, and multimodal target distributions (Vrugt et al., 2008), such as the ones provided for measurements of several days of sap flow used in the objective function of this study.

Two sets of optimisations were executed with DREAM for Q.i. and Q.p. as follows: (i) calibration of stem flow $\left(F_{\text {stem }}\right)$ to match the sap flow measurements, and (ii) calibration of root flow $\left(F_{\text {root }}\right.$ and $\left.F_{\text {rootTg }}\right)$ to match the results found in the isotope experiments. Each calibration attempt was evaluated using the FITEVAL framework (Ritter et al., 2011), which consists of a goodness-of-fit evaluation protocol. The procedure combines the calculation of various error indexes to evaluate the performance of the model versus real measurements and produces a ranked output under the four categories: "Very Good", "Good", "Acceptable" and "Unsatisfactory".

Table 5.2: Initial guess for parameter optimisation of resistances and capacitances, together with optimised values for DRY and WET. Capacitances $\left(C_{\text {pool }}\right)$ units MPa g ${ }^{-1}$ and Resistance $\left(R_{\text {pool-pool }}^{x}\right)$ units MPa s g ${ }^{-1}$

\begin{tabular}{llll}
\hline & Parameter & WET & DRY \\
\hline Initial guess & $C^{*}$ & 940 & 719 \\
Optimised & $C_{\text {crown }}$ & 2100 & 1470 \\
& $C_{\text {stem }}$ & 11576 & 8010 \\
& $C_{\text {root unsat }}$ & 120 & 84 \\
& $C_{\text {root sat }}$ & 30 & 21 \\
\hline Initial guess & $R^{x *}$ & 1072 & 1087 \\
Optimised & $R_{\text {stem-crown }}^{x}$ & 2448 & 4695 \\
& $R_{\text {root unsat-stem }}^{x}$ & 75 & 144 \\
& $R_{\text {root sat-stem }}^{x}$ & 24 & 46 \\
\hline
\end{tabular}

* from Steppe et al. (2008)

\subsection{Results and discussion}

\subsubsection{Naturally abundant isotopes in the soil-plant compartments}

The results of this section are based on the mixing model described in Eq. 5.4, which assumes that the isotopic composition in the sap is the 
result of the mixing of water from soil water at 25, 50, 75 and $100 \mathrm{~cm}$ deep, and from the groundwater.
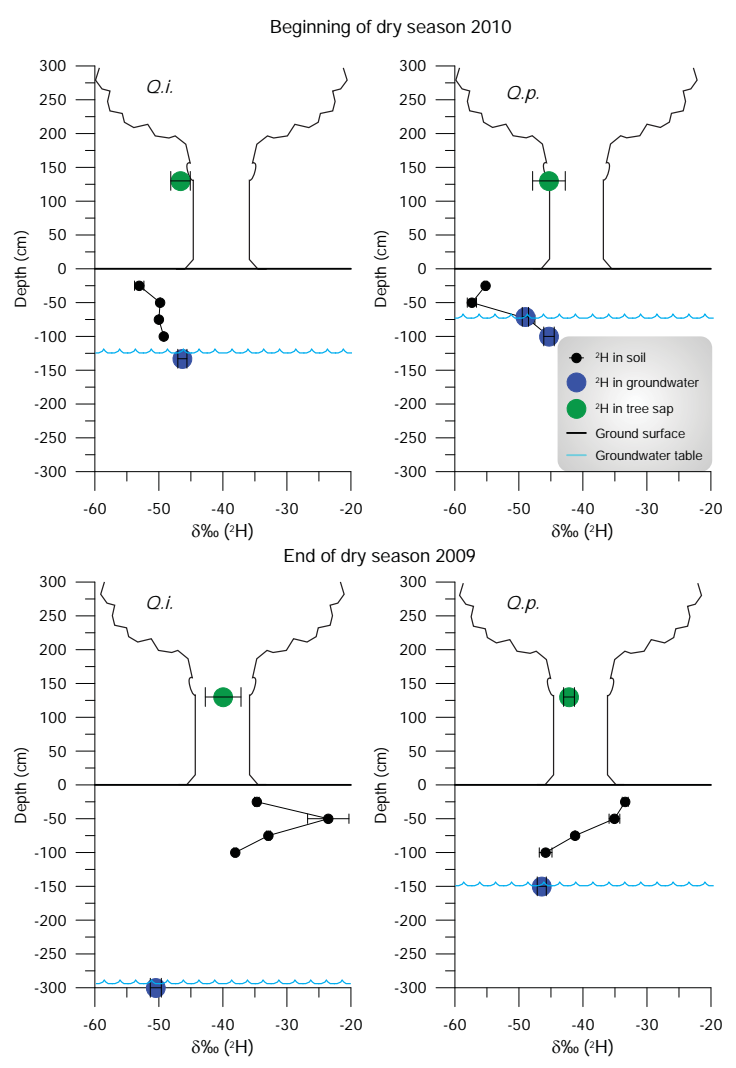

Figure 5.5: Average isotopic readings from two subsurface sources (soil layers and groundwater) and from the xylem of tree compartments representing an isotopic mixture, measured in the early period of the dry-season referred to as WET (May-June 2010) and in the late period of the dry-season referred to as DRY (August-September 2009) on a Q.i. tree (a. and c.) and on a Q.p. tree (b. and d.). The error bars show the standard deviation. $0 \mathrm{~cm}$ in the y axis refers to the ground level.

The results are presented starting with the wetter period of the dry season (referred to as WET), and then progressing to the driest period of the dry season (referred to as DRY). However, this does not reflect a continuous time line, because WET measurements were made in MayJune 2010, whereas DRY measurements were made in August-September 2009.

Discussing the results in this order facilitates the explanations of the observed patterns and does not affect the results because: (1) both years had comparable rainfall regimes, (2) the focus of the analysis was set on the dynamics triggered by the scarcity and/or abundance of water rather 
than on temporal variability, and (3) climatic conditions in dry season for this area are rather homogeneous (Lubczynski and Gurwin, 2005).

Average measurements for a 24-hour $\delta^{2} H$ sampling of the considered water compartments (i.e. soil layers, groundwater and xylem) in 2009 and 2010 are illustrated in Figure 5.5. These unprocessed measurements already give initial suggestions of the origin and contributions of water sources at the beginning and at the end of the dry season. The $2010 \delta^{2} H$ measurements, referred to as the WET period of the dry season, show that groundwater for both species (blue circle) had a $\delta^{2} H$ that was not significantly different from the average $\delta^{2} \mathrm{H}$ isotopic values for the 50 to the $100 \mathrm{~cm}$ deep soil layer for Q.i., and for the 75 to the $100 \mathrm{~cm}$ soil layer for Q.p. Moreover, the $\delta^{2} H$ isotopic value of the water in the sapwood was closer to the $\delta^{2} H$ isotopic values of the deep soil layers and the groundwater than to the shallower layers $(<50 \mathrm{~cm})$. The $\delta^{2} H$ isotopic pattern observed in different compartments for the WET season seems to be related to shallow groundwater for both species, especially for Q.p., which is generally located where the water table is the shallowest (see Chapter 4).

In the case of $\delta^{2} H$ measurements in 2009, referred to as the DRY period of the dry season, the $\delta^{2} H$ of groundwater was significantly lower than the $\delta^{2} H$ for all the investigated soil layers in $Q . i$. In the case of Q.p. the $\delta^{2} H$ groundwater measurements were significantly lower just for the 25 to $75 \mathrm{~cm}$ deep soil layers. This indicated that the unsaturated zone was more isotopically enriched so more exposed to evaporation $(E)$, and that most likely groundwater had a lower effect on the $\delta^{2} H$ isotopic composition of the xylem during the DRY period than during the WET period, when the $\delta^{2} H$ values for the soil layers were very close or identical to those for the groundwater. The $\delta^{2} H$ isotopic composition of the first $75 \mathrm{~cm}$ of soil layers during the DRY period (up to $100 \mathrm{~cm}$ for $Q . i$.), is most likely influenced by the drying forces (i.e. soil water evaporation) that have enriched the $\delta^{2} H$ isotopes during several months of rainfall absence, and thus provides enough differentiation to obtain accurate results from mixing models (Dawson et al., 2002)

The similarity of the $\delta^{2} H$ groundwater values to the sapwood values during the WET period, while being indicative, made difficult to establish the contribution of the sources as compared to the mix. Despite of this constraint, the calculations for the WET season converged in the MixSIR approach for most of the samples.

Interestingly, the $\delta^{2} H$ values of sapwood in both trees showed low variation from the WET to the DRY period, in spite of the sharp changes observed in the $\delta^{2} H$ values of shallow soil layers. Moreover, the $\delta^{2} H$ values of the sapwood were also closer to the $\delta^{2} H$ values of the groundwater than to those of the soil layers during the DRY and WET period. These are initial indications of the importance of groundwater sources for the hydrological dynamics of both species.

The isotopic measurements of David et al. (2007) for Q.i. in midAugust 2002 (surface soil $-19.90 \% \delta^{2} H$, subsurface soil $-42.83 \% 0 \delta^{2} H$, groundwater $-28.81 \% \delta^{2} H$ and twig-water $-32.52 \% \delta^{2} H$ ) were mostly 
comparable to the $\delta^{2} H$ measurements obtained in this study for $Q$.i. in 2009 (see Figure 5.5), except for the groundwater, which was significantly higher in their case, probably due to differences in groundwater depth (4-5 $\mathrm{m}$ in their study) and soil type (Dystric Cambisol). This was rather surprising because agreement was expected between $\delta^{2} H$ groundwater measurements of this study and theirs, as such measurements were taken in the Mediterranean area of Portugal with almost the same climatic conditions and vegetation as in this study. Such difference might be explained by the distance to the ocean which may have influenced isotope signatures. Nevertheless, despite some differences between the measurements of David et al. (2004) and the ones in this study - suggesting high spatial variability of $\delta^{2} H$ isotopic values across the regions - the patterns of both were comparable.

The $\delta^{2} H$ values of Zencich et al. (2002) in Banksia tree species (surface soil -28.4\%o $\delta^{2} H$, subsurface soil - $17.6 \% 0 \delta^{2} H$, groundwater - $15.6 \% \delta^{2} H$ and twig-water $-15.8 \% \delta^{2} H$ ) were, however, mostly higher than the measurements for either Q.i. or Q.p. The differences with the measurements of Zencich et al. (2002) were expected because the $\delta^{2} H$ measurements were taken in another continent with different climatic regimes and lithological conditions (groundwater depth varied from $2.5 \mathrm{~m}$ to $10 \mathrm{~m}$ depending on the area), and although the differences are larger, the pattern is similar and in agreement with the obtained results of this study for the DRY season.

\subsubsection{Mixing-models results}

The daily water contribution estimates for each of the considered compartments at the beginning and at the end of the dry season can be seen in Table 5.3. This was the result of compiling the 24-hour estimates, for Q.i. and Q.p. using both MixSir and Iso-source.

The average water contribution to the sap water in the Q.i. tree during the WET period was $96 \%$ from the groundwater $(s t d .+/-3)$, as compared with the $4 \%(s t d .+/-1)$ contribution from soil water considering all the analysed soil layers together (25 $\mathrm{cm}$ to $100 \mathrm{~cm}$ depth). In the case of Q.p. during the WET period, the water table during the isotope sampling was at $75 \mathrm{~cm}$ depth, thus soil layers at $100 \mathrm{~cm}$ and $75 \mathrm{~cm}$ depth were both completely saturated. Therefore, for the WET period the $75 \mathrm{~cm}$ to the $100 \mathrm{~cm}$ soil layers and the groundwater were considered as the same water compartment. In this way, the average groundwater contribution to the sap water was $98 \%(s t d .+/-1 \%)$ and $2 \%(s t d .+/-1 \%)$ from the rest of the analysed unsaturated soil-layers together (25 to $50 \mathrm{~cm}$ depth).

The average contributions in Table 5.3 are a clear indication that Q.i. and Q.p. are phreatophyte species but more importantly, that they abstract most of their water from the shallow groundwater during the WET period of the year. This is an interesting result because it was expected that in WET period Q.i. and Q.p. trees would use the available water in the shallow soil in higher proportions, (i.e. a much higher contribution from the unsaturated soil), which was not the case. 
Table 5.3: Average source contributions to sap-water calculated from Eq. 5.4 , for the 24 hours sampling at 4 soil-water layers and groundwater derived from $\delta^{2} H$ mixing models analysed with Iso-source, in WET period of dry season 2010 (Mixir) and DRY period of dry season (IsoSource) 2009.

\begin{tabular}{|c|c|c|c|c|c|c|c|c|c|c|}
\hline \multicolumn{11}{|c|}{ *WET beginning of dry season 2010 (May-June) } \\
\hline & \multicolumn{5}{|c|}{ Q.i. } & \multicolumn{5}{|c|}{ Q.p. } \\
\hline & $25 \mathrm{~cm}$ & $50 \mathrm{~cm}$ & $75 \mathrm{~cm}$ & $100 \mathrm{~cm}$ & GW & & $25 \mathrm{~cm}$ & $50 \mathrm{~cm}$ & $\mathrm{GW}^{* *}$ & \\
\hline Mean & 0.01 & 0.01 & 0.01 & 0.01 & 0.96 & & 0.01 & 0.01 & 0.98 & \\
\hline Min. & 0.00 & 0.00 & 0.00 & 0.00 & 0.90 & & 0.00 & 0.00 & 0.97 & \\
\hline Max. & 0.02 & 0.03 & 0.03 & 0.03 & 0.99 & & 0.01 & 0.01 & 0.99 & \\
\hline Med. & 0.01 & 0.01 & 0.01 & 0.01 & 0.97 & & 0.01 & 0.01 & 0.98 & \\
\hline Std. & 0.01 & 0.01 & 0.01 & 0.01 & 0.03 & & 0.00 & 0.00 & 0.01 & \\
\hline \multicolumn{11}{|c|}{ *DRY End of dry season 2009 (August-September) } \\
\hline & \multicolumn{5}{|c|}{ Q.i. } & \multicolumn{5}{|c|}{$Q . p}$. \\
\hline & $25 \mathrm{~cm}$ & $50 \mathrm{~cm}$ & $75 \mathrm{~cm}$ & $100 \mathrm{~cm}$ & GW & $25 \mathrm{~cm}$ & $50 \mathrm{~cm}$ & $75 \mathrm{~cm}$ & $100 \mathrm{~cm}$ & GW \\
\hline Mean & 0.15 & 0.09 & 0.14 & 0.19 & 0.43 & 0.09 & 0.10 & 0.18 & 0.31 & 0.32 \\
\hline Min. & 0.06 & 0.04 & 0.06 & 0.08 & 0.15 & 0.06 & 0.07 & 0.13 & 0.23 & 0.23 \\
\hline Max. & 0.24 & 0.15 & 0.22 & 0.26 & 0.76 & 0.14 & 0.15 & 0.25 & 0.37 & 0.43 \\
\hline Med. & 0.15 & 0.09 & 0.14 & 0.19 & 0.43 & 0.08 & 0.10 & 0.18 & 0.31 & 0.32 \\
\hline Std. & 0.05 & 0.03 & 0.04 & 0.05 & 0.17 & 0.02 & 0.03 & 0.04 & 0.04 & 0.05 \\
\hline
\end{tabular}

${ }^{*}$ The values indicate contributing fractions ${ }^{* *} \mathrm{GW}$ for $Q . p$. during the WET period consists of two samples for the groundwater.

The results suggest that in this particular catchment (see Chapter 3 for more details on the study area), Q.p. is behaving as a riparian species (see Chapter 4), thus abstracting most of its water from the shallowest groundwater, (i.e. close to ephemeral and main rivers).

The average water contribution to the sap water in Q.i. during the DRY period was $43 \%$ from the groundwater (std. $+/-17$ ) and $57 \%$ (std. $+/$ - 17) contribution from soil water considering all soil layers together (25 $\mathrm{cm}$ to $100 \mathrm{~cm}$ depth). In the case of Q.p. during the same period, the average water contribution from the groundwater to the sap water was $32 \%$ (std. $+/-1 \%)$, as compared with the 31\% (std. +/- 1\%) contribution from the $100 \mathrm{~cm}$ depth soil layer, and the $37 \%(s t d .+/-1 \%)$ contribution from soil water considering the rest of soil layers together $(25$ to $75 \mathrm{~cm})$.

It was established that during the DRY period the water table was at $300 \mathrm{~cm}$ depth for Q.i. and at $150 \mathrm{~cm}$ depth for Q.p. Therefore, by following the $\delta^{2} H$ measurements (Figure 5.5c) and the contribution estimates (Figure $5.6 \mathrm{c}$ and d), it was observed that the soil layers at 25 to $100 \mathrm{~cm}$ depth for Q.i. were not saturated with water (confirmed also by soil moisture measurements; see Chapter 3 for further details), and thus the change in $\delta^{2} H$ values was the result of enrichment (increment in the higher $\delta^{2} H$ concentration due to water evaporation) by the drying front established in the soil.

For Q.p., the 25 to $50 \mathrm{~cm}$ depth soil layers had a higher concentration 

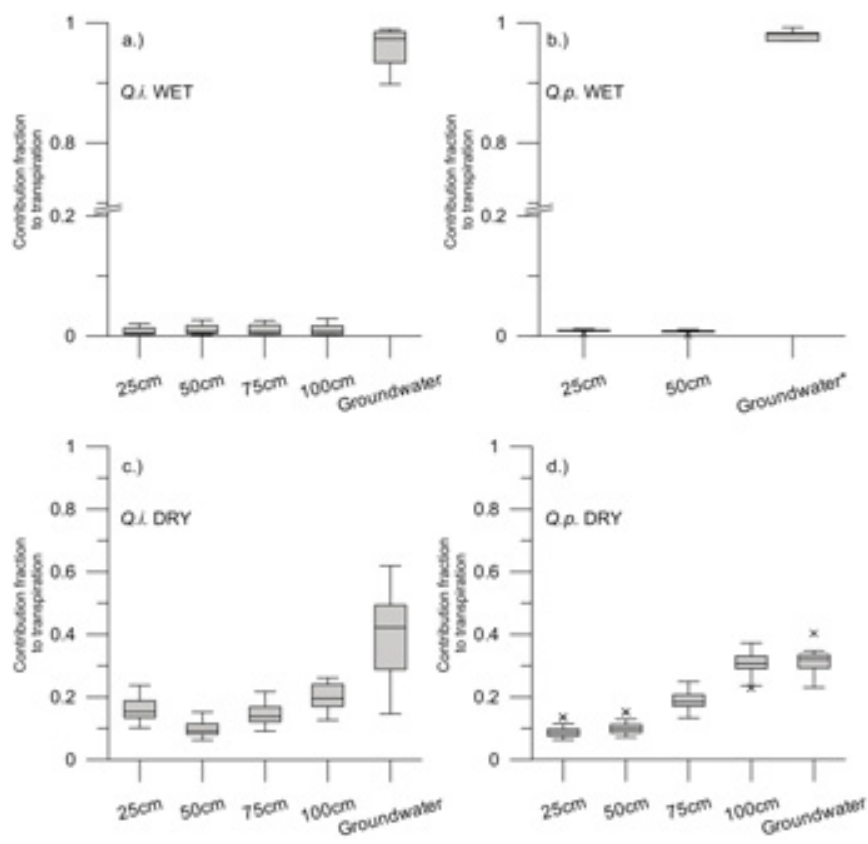

Figure 5.6: Boxplot of the contribution fraction for a.) Q.i. and b.) Q.p. in the WET period (June 2010); and c.) Q.i., and d.) Q.p. in the DRY period (August-September 2009).

of $\delta^{2} H$ than the rest measurements (see $5.5 \mathrm{~d}$ ); even higher was $\delta^{2} H$ of Q.i. at $50 \mathrm{~cm}$ depth, both most likely influenced by the drying front in the soil. In the case of soil layers at 75 to $100 \mathrm{~cm}$ depth in Q.p., the water table was closer to the $100 \mathrm{~cm}$ depth layer. It is then feasible that $\delta^{2} H$ values for these soil layers were likely less influenced by the drying front, and instead more influenced by moisture brought by the capillary rise from the groundwater. This was especially relevant for the $100 \mathrm{~cm}$ depth soil layer, where $\delta^{2} H$ values were not significantly different from the groundwater values (see 5.5d). Therefore, when reinterpreting the isotopic tracing analysis by aggregating the $\delta^{2} H$ contribution at $100 \mathrm{~cm}$ depth to the $\delta^{2} H$ contribution of the groundwater, it is then concluded that $63 \%(s t d+/-1 \%)$ of the water abstracted by Q.p. comes from the saturated zone of the soil.

The identified water source contributions of the unsaturated and saturated zones to transpiration in Q.i. are in agreement with the findings for the end of the dry season (DRY period), published by David et al. (2007), who also used naturally abundant isotopes. Their result indicated that $70 \%$ of the water abstracted by Q.i. was from the groundwater, which is well within the $15 \%-76 \%$ groundwater contribution range found in this study for the same period. This range seems large but, as discussed in Subsection 5.3.3, this is due to the temporal variability of the groundwater contribution. Unfortunately, David et al. (2007) did not measure $\delta^{2} H$ 
concentrations during the wetter part of the dry season (WET period) so we cannot compare findings in this respect. However, as the study areas, groundwater depth, and species are very similar, it is likely that they also would have the highest groundwater contribution at the beginning of the dry season (WET period), provided they would have the same shallow water table conditions.

In the case of Q.p., to the author's knowledge, there are no naturally abundant tracing studies indicating the water source contributions to transpiration. However, the first indication of deep-soil water abstraction by Q.p. was addressed by Hernández-Santana et al. (2008c) in their soil moisture and water storage measurements in a Mediterranean closed forest.

In general, previous studies have concluded that groundwater abstraction is a process triggered by low moisture in the unsaturated soil-layers (Hultine et al., 2004) and, as such, groundwater abstraction for Q.i. and Q.p. was expected to be higher towards the end of the dry season (DRY period) when the soil has reached the lowest moisture of the year. This was not the case for the studied Q.i. and Q.p. trees, as the highest consumption was reached at the beginning of the dry-season (WET period) (see Figure 5.7, for an example of a soil profile during the dry season).

The observations of Hultine et al. (2004) fit well with situations where the groundwater table is not directly influencing the first metre of unsaturated soil layers and continues reducing its depth throughout the season. However, in the Sardón catchment groundwater is so shallow that it is within the reach of the tree roots early in the dry-season (i.e. WET period). Moreover, moisture in the unsaturated zone layers are most likely influenced by the capillary rise of the groundwater.

There are diverse reasons explaining why such a situation is possible in the Sardón catchment: (1) Q.i. and most notoriously Q.p. are behaving like riparian/phretophytic species, meaning that their roots are for the most part of the year in contact with the water table linked to permanent or ephemeral rivers; (2) species of the genus Quercus, such as Q.i. and Q.p. naturally develop deep root systems of more than $3 \mathrm{~m}$ deep (Silva and Rego, 2003; Canadell et al., 1996) and most likely these root systems tolerate anoxic conditions for some periods of the year as other riparian species do (e.g. Eucalyptus camaldulensis (Sena Gomes and Kozlowski, 1980; Blom and Voesenek, 1996)); (3) groundwater in the Sardón catchment is so shallow that it is easily accessible for abstraction in most parts of the catchment; (4) as the water table recedes there is moisture in the unsaturated layers that can be abstracted, whereas groundwater is deeper and thus more difficult to abstract; thus (5) groundwater depth and not soil moisture seems to be the main source determining water abstraction dynamics for Q.i. and Q.p. in Sardón These observations fit well with findings and hydrological explorations concerning other riparian species (Baird and Maddock, 2005).

Another important aspect to consider, in order to fully establish the dynamics governing the water flow in Q.i. and Q.p., is the role of superficial and deep roots. Studies on species of the genus Quercus 


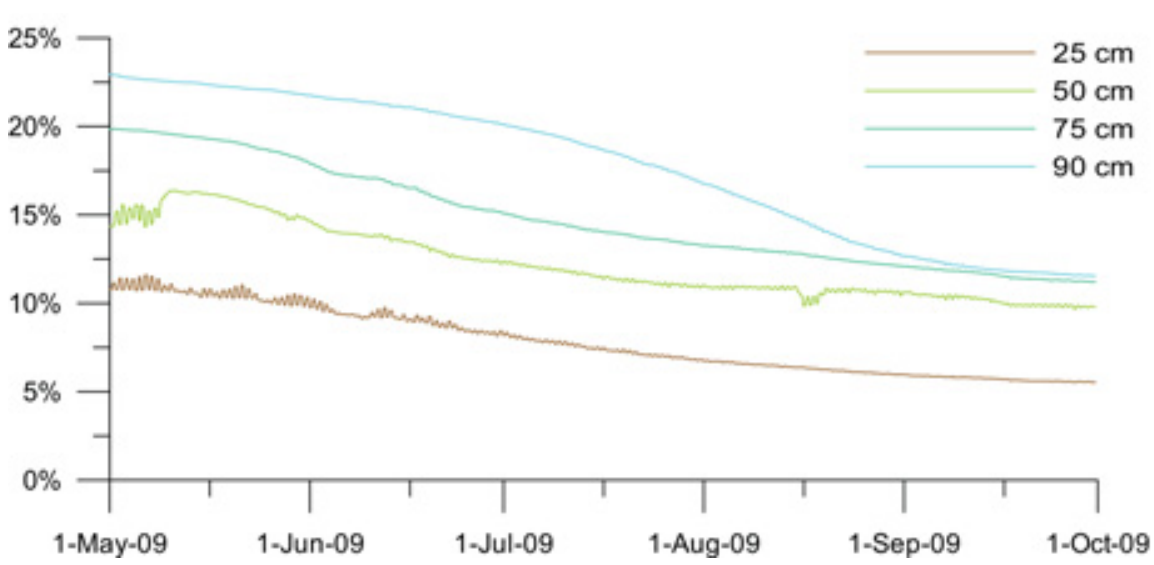

Figure 5.7: Soil moisture profile at four depths for a Q.p. tree monitored during the fieldwork campaign of 2009. Y axis represents \% of water content in the unsaturated layers. This tree was located further from the river and thus its profile differs slightly from that of the Q.p. tree in which the isotope tracing was made. However, it does show how deeper soil layers (75 to $90 \mathrm{~cm}$ deep) are kept with relatively high moist throughout the dry season, and how only at the very end (of the DRY period) the effect of the shallow groundwater diminishes and drier conditions prevail.

have demonstrated the importance of the connection between waterflow dynamics of roots and groundwater. For example, David et al. (2013) using a combination of isotopes tracing, sap-flow measurements and a modelling framework (a similar design to the one of this study) highlighted specific water-use strategies such as hydraulic-lift and ascend for Quercus suber L. Preliminary explorations on root flow measurements on Q.i. and Q.p. carried out by the author (data not shown), did not indicate hydraulic-lift or ascend. However, more studies are required to define the role of roots for Q.i. and Q.p. in the context of shallow groundwater and granitic soils as in Sardón, and thus aid on the definition of a proper modelling representation.

The contribution proportions for Q.i. and Q.p. in the WET and DRY periods demonstrate the following: (1) water contributions to the transpiration are temporally variable; for example, groundwater contribution decreased towards the end of the dry season (from 95\% to $43 \%$ and from $98 \%$ to $63 \%$, for Q.i. and Q.p. respectively), so the contribution of the unsaturated zone significantly increased in the same period; (2) Q.i. and Q.p. trees absorb most of the water from the groundwater and deep soil layers in the capillary fringe; and (3) the change in water table depth throughout the dry season determines the water contributions to transpiration by the unsaturated and saturated zones. 


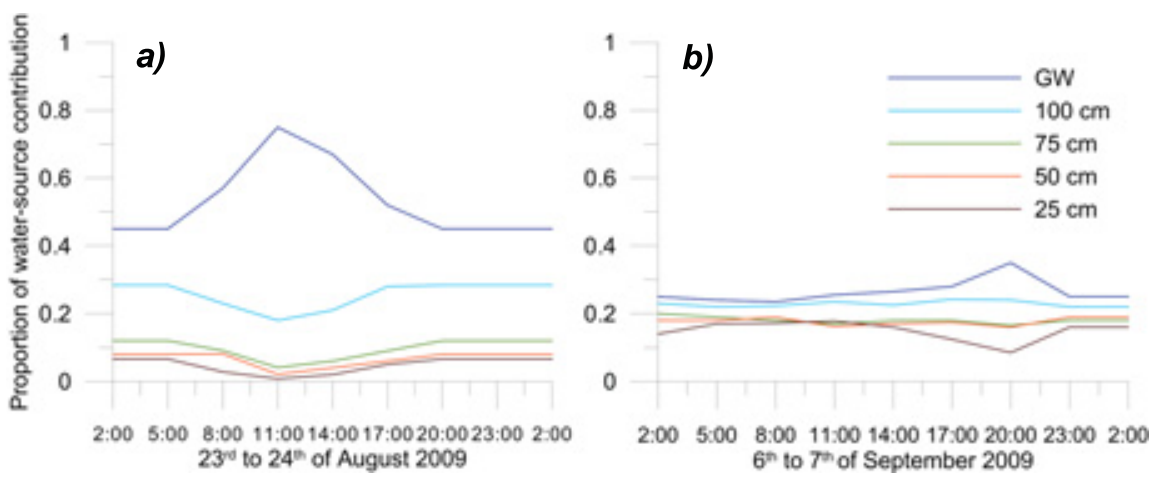

Figure 5.8: Time series of feasible contributions from each source to the sap water extracted from the tree every three hours (three samples per hour), in (a) Q.i. and (b) Q.p.. The time follows GMT+1 Spanish summer time, which is $2.5 \mathrm{~h}$ ahead as compared with geographical (solar) time.

\subsubsection{Sourcing hourly variability}

The source contribution results obtained from the 24-hour sourcing experiments varied hourly in the DRY period only, but most clearly for the groundwater contribution, as seen in Figure 5.8. The range of feasible proportional contributions for Q.i. (Figure 5.8a) increased from 0.42 at 05:00 to 0.75 at 11:00 hours, then decreasing to reach 0.42 by 20:00 hours.

For Q.p. the proportion followed a less pronounced temporal variation (Figure 5.8b). The range of feasible source contributions at 11:00 hours did not indicate a significant predominance of any of the sources. All sources (unsaturated zone layers and groundwater) contributed $\approx$ 20\% of the mix. This tendency was reversed at 20:00 hours, when the groundwater displayed a higher but non-dominant contribution (35\%). This overlap of source contributions for Q.p. indicates that the system was less constrained than for Q.i. (Figure 5.8). This situation is normally observed during very humid conditions (rainy season or winter-time) (Phillips and Gregg, 2003; Zencich et al., 2002), when there is more precipitation and the sub-surface layers are less depleted, and thus isotopic compositions of water sources are very similar. As we performed our experiments at the peak of the dry season, this was not the case. However, Q.p. in the Sardón catchment tends to be located in areas closer to streams and river valleys (see Section 4.3.6 for details on the spatial patterns), where soil is more moist due to the shallow depth of the water table, thus explaining the observed relatively uniform contribution of sources in Figure 5.8b.

The identified temporal variation provides new evidence of the dynamics of deep water abstraction as it was determined that it is not static in time. By comparing the contribution results for groundwater (blue line in Figure 5.8b) and sap flow data (brown line in Figure 3.9 in Chapter 3), 
it was found that the deep-water abstraction of Q.p. reaches a maximum of two hours after the maximum peak of transpiration, and decreases towards sunset. Moreover, the hourly variability results provided additional support for the proposed water-use strategies described in Chapter 3 and discussed by Hernández-Santana et al. (2008a) and Mediavilla and Escudero (2003). The water-use strategy during the dry season proposed for Q.i. (see Chapter 3 and Chapter 4) states that it uses a conservative approach; therefore it copes better with water-scarcity scenarios where the water table is generally deeper. In contrast, Q.p. has a non-conservative water-use strategy, because it has a limited time during the year (May to September approx.) to complete a reproductive cycle before going dormant. Therefore it is more likely to find Q.p. located in areas where it can abstract and transpire as much water as possible, generally where the water table is shallow enough so it can continuously abstract water from all soil layers.

\subsubsection{Explaining water contribution patterns with a tree water flow model}

\subsubsection{Model calibration and validation for daily sap flow}

In the following sections the modelling results for $Q . i$. alone are discussed to avoid repetition. This is because the results for Q.i. and Q.p. were comparable and thus findings and insights obtained from the modelling exercise were almost identical and thus pertaining to both species.

Five days of sap flow measurements of Q.i. were used as the calibration target while optimising selected parameters (i.e. resistances and capacitances) with the DREAM algorithm (see Section 5.2.2.5 for calibration procedures and Tables 5.4 and 5.4 for a list of optimised parameters). DREAM converged after $\approx 7000$ iterations and the estimated parameters produced outputs that resembled the measured sap flow data. The evaluation of the performance using the mean absolute percentage error (MAPE) indicated a non-satisfactory fit for the hourly data (70\%), but a satisfactory fit for the daily averaged sap flow (20\%) during the modelled period (20 days). Despite the low hourly fit, the variation patterns of the modelled sap flow in general resembled the sap flow measurements (see Figure 5.9a).

The reason behind the low fit of the hourly data is that the modelled water flow in the stem had a higher daily variation than the sap flow measurements. This variation was the result of the influence of vapour pressure deficit (VPD) on the simulated sap flow, to which the model was highly sensitive. Other climatic factors influenced the process but not as much as the VPD, as it can be observed in Figure 5.9b. The influence of VPD on the daily variation patterns of the modelled sap flow is evident in the match of daily sap flow (Figure 5.9a) and VPD peaks (Figure 5.9b).

It was attempted to lower the sensitivity to VPD by increasing crown resistance $\left(R_{\mathrm{air}}^{x}\right)$, as if simulating stomata closure. This indeed smoothed 


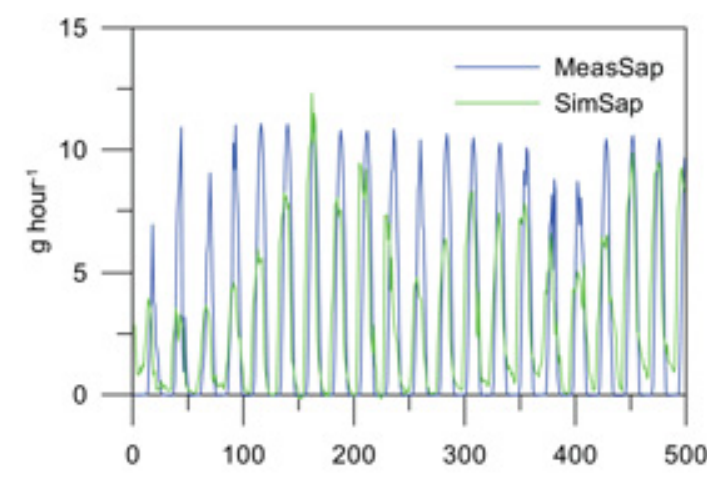

a
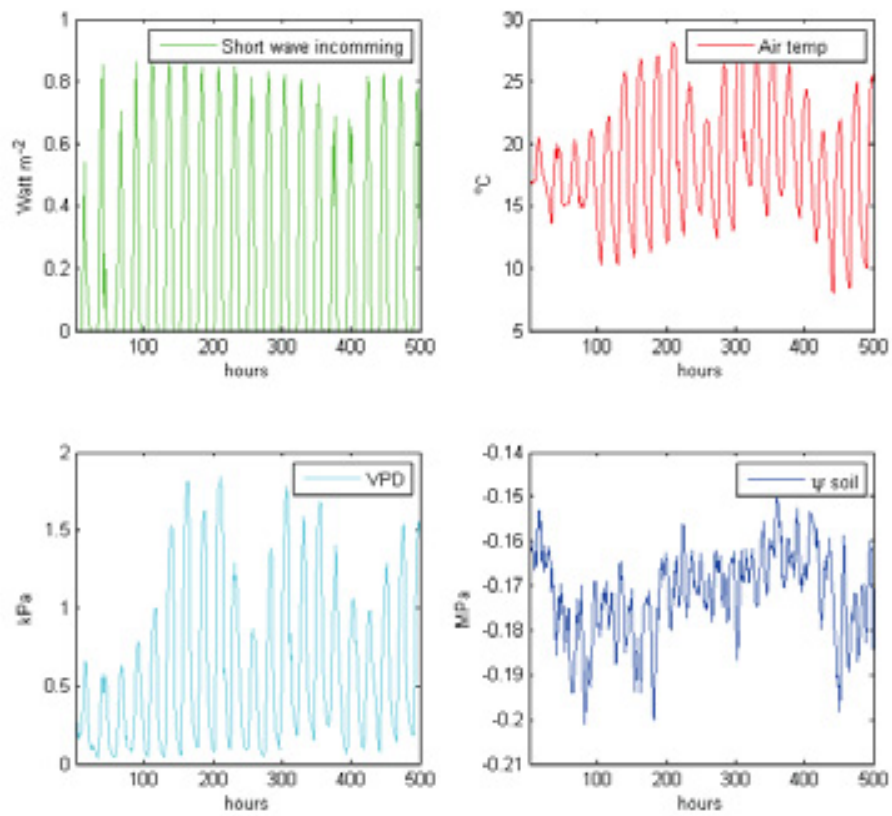

b

Figure 5.9: Figure (a) illustrates the field sap flow data (MeasSap) and the simulation after convergence (SimSap). Figure (b) shows the climatic drivers used for simulation, shortwave incoming radiation (green line), air temperature (red line), vapour pressure deficit (VPD) (cyan line) and soil water potential at $50 \mathrm{~cm}$ (dark blue line). 
out the water flow output but was not sufficient to resemble the uniformity observed in the field measurements (Figure 5.9a).

The low fit of the hourly patterns is not a major drawback when it comes to the model's performance in explaining daily sap flow or sourcing contributions indicated by $\delta^{2} H$ values (discussed in the subsection below). It just revealed that the structure and conceptualisation of the tree water flow model can be improved by representing the stomata closure mechanisms influencing sap flow rates. An extra module linking such mechanisms might be used in future studies if accurate hourly simulations are required.

\subsubsection{Modelling water contribution from the unsaturated and saturated zones of the soil}

In order to calibrate the model to properly represent the unsaturated and saturated abstraction proportions for Q.i., the DREAM algorithm was set to calibrate the resistances in the modelled compartments to match calculations of sap flow measurements broken down into the unsaturated and saturated elements (i.e. sap flow rates times the average source contributions indicated by the isotopes sourcing). This was done twice, one for the DRY period and then for the WET period. Thus resistances parameters were calibrated to fit $\approx 40 \%$ of the water originating from deep sources in the DRY period and $>90 \%$ in the WET period.

The results of the optimisation for the DRY conditions showed that by increasing the resistance of the roots of the unsaturated compartment two times, the contribution from the saturated compartment increased to $40 \%$ (see Figure 5.10a), matching the targeted source contributions. This optimisation indicates that the model can properly reproduce water potential/resistance conditions in the tree and soil compartments during the DRY period, and that the calibrated parameters represent the water flow conditions for tapping water from saturated layers. The success of the optimisation relied on high temporal resolution measurements of sap flow and climatic factors that provided enough information about the data variability to adequately represent hourly and daily dynamics.

In the case of the WET season the optimisation was more complex. First it was attempted to generate a contribution of $90 \%$ from the saturated zone of the soil. The DREAM algorithm then converged to an unrealistically high root resistance value in the unsaturated zone, due to the resistance principles embedded in the structure of the model. This converged value was unrealistic, not just because it was too high, but also because it would have meant that during the WET period the unsaturated zone of the soil was extremely dry. This was obviously not the case in the WET period, when the soil moisture was relatively higher $(13 \%$ as measured in the field, see Chapter 3 for more details) due to rainfall regimes in late spring and the proximity to the shallow groundwater.

Therefore, it was decided to optimise the model to simulate conditions for which the root resistances of the unsaturated and saturated zones would be almost identical, meaning that the roots located in both 


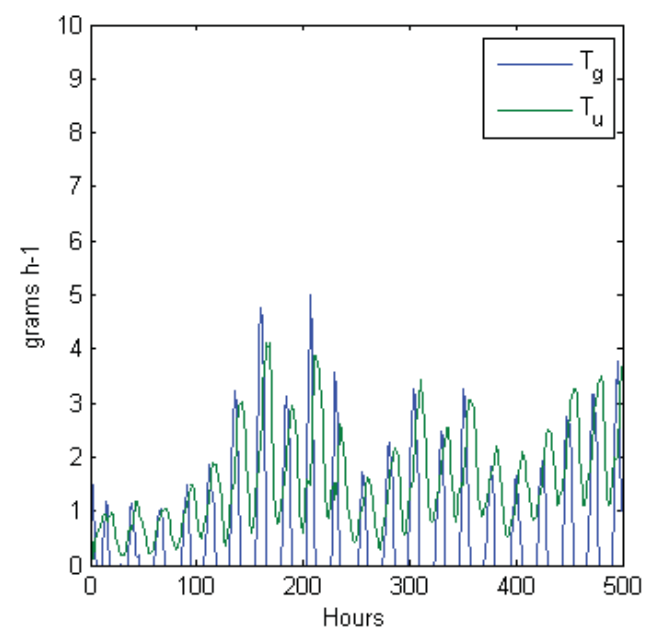

a

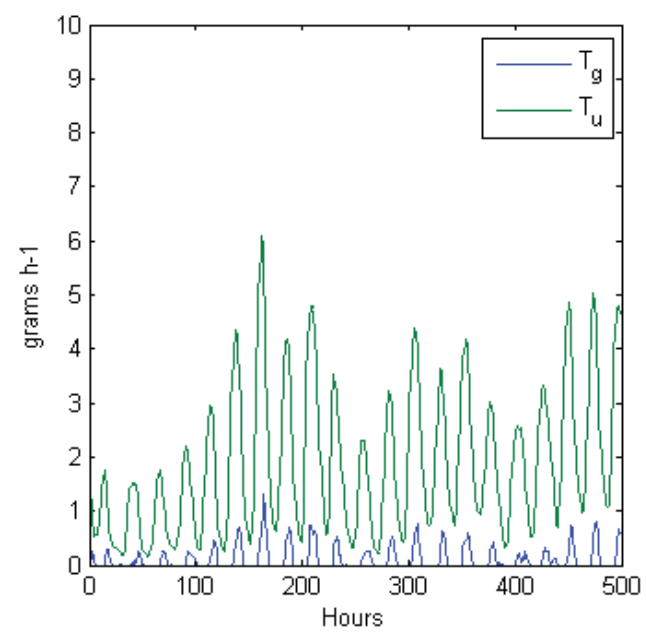

b

Figure 5.10: Saturated zone $\left(T_{g}\right)$ and unsaturated zone $\left(T_{u}\right)$ contribution simulations for a typical Q.i. tree with a $23 \mathrm{~cm}$ diameter at breast height under soil moisture conditions of the (a) DRY and (b) WET periods. 
zones would have almost the same water availability. This scenario better resembled the observed soil water conditions during the WET season, with shallow groundwater conditions. During this period the soil moisture and water potential were high across the studied soil layers. This means that the unsaturated zone was in hydraulic contact with shallow groundwater through the capillary fringe.

Under these conditions the model converged to a scenario where $>90 \%$ of the transpired water was abstracted from the first compartment of the unsaturated zone (see Figure 5.10b). This result seems contradictory to the contributions found for the WET season with the $\delta^{2} H$ sourcing indicating 90\% abstraction from groundwater for Q.i. The reason for this contradiction, by following the model rationale, lies on the conditions of shallow groundwater $(<100 \mathrm{~cm})$ and the influence of the water table and the capillary rise on the unsaturated zone that increases the water availability in that compartment, hence creating small differences between the root resistances in the unsaturated or saturated zones. Under such conditions, the model reacts by using the root compartment closest to the stem, in this case mostly the shallowest root compartment (i.e. the unsaturated compartment). What this indicates is that trees during the WET period "by-bass" the thin unsaturated layers and mostly abstract water from the groundwater.

Such dynamic can be applied to most trees where the groundwater is shallow in the WET period, but there are sectors in the Sardón catchment (e.g. water divide) where this might not be the case. Initially the research aimed to study trees in those areas as well, however the subsurface in the Sardón catchment is of mostly granitic origin which made fieldwork activities rather difficult. As we could not install piezometers in regions where trees had their roots directly on the granite, we selected trees closer to the valley.

In areas closer to water bodies (ephemeral or not) water table is normally shallower than the average for the catchment thus explaining why the model worked best for the DRY period but not for WET when the species are facing riparian conditions (e.g. highly saturated subsurface due to shallow groundwater). Therefore, the modified water-plant model of Steppe et al. (2006) used in this study is unfortunately not adequate to emulate water potential conditions for a $>90 \%$ groundwater contribution to transpiration in shallow groundwater condition $(<100 \mathrm{~cm})$. The reason for this is that the model is controlled by soil moisture/water potential only and does not account for the location and concentration of roots in the subsurface. Moreover, it does not account for the effects of a shallow water table on the roots of trees, such as the lowering of root resistances due to higher moisture. However, it is possible the model would work during WET period on trees located in areas where the water table is > 1.5 metres, as is the case in the upper part of the catchment.

Despite these shortcomings, a further modification of this model to include the effect of a shallow water table, as in the model of Baird and Maddock (2005) for riparian species, would probably generate more realistic simulations and allow to represent variety of water sourcing 
scenarios for the trees. Another step is to undertake careful analysis of the rooting connections and root-flow dynamics as proposed by David et al. (2013) This will allow to better connect the mechanisms of control operating at the soil and in the stem, while defining the movement of water through the root compartments.

\subsubsection{Flow from storage and water potential simulations}

Calibration of the circular variability module (see Appendix in 5.5) was attempted using available dendrometric measurements taken in the first week of September 2011 for both Q.i. and Q.p. to match as closely as possible the conditions of September 2009. However, the DREAM algorithm did not converge to a satisfactory value using these measurements. The cause for this is likely related to the close connection between circular variability and water availability and climatic conditions, which unfortunately did not completely match our initial 2009 measurements. Nevertheless, the observations from circular variability simulations provide interesting insights that are worth sharing even if the diametric magnitudes were not validated.

The results of the simulation for a Q.i. tree, with a DBH of $23 \mathrm{~cm}$ and a $3 \mathrm{~m}$ high stem, indicated that water flowing in and out of the storage compartments was closely synchronised with the diameter variation of the trunk and with the water potentials of the considered plant compartments. This process is clearly observable in Figure 5.11, where a close-up on three selected days is illustrated.

For the DRY-period simulation (Figure 5.11a), the daily-sap flow variation of Q.i. followed a pattern similar to that of VPD (in Figure 5.11c black line is VPD) as discussed in Subsection 5.3.4.1. For the WET-period simulation in Figure 5.11b, the xylem flow, storage flow and diameter variation also followed the VPD pattern, but it was less pronounced and xylem flow slightly lower than during the DRY season.

This VPD influence was also observed in various compartments of the model. Figure 5.11c in the DRY period shows a decrease in magnitude in the flow originating in the shallow (green line) and deep roots (blue line) of the tree, for a period when the VPD was decreasing as well. For the WET period, Figure 5.11d shows a similar pattern but also a decrease in magnitude in the flow originating in the shallow roots (green line) and almost no flow from the deep roots (blue line) of the tree.

Figure 5.11e illustrates the inward (positive) and outward (negative) water flow from the storage compartments, and how it diminishes following the VPD reduction as well. In the case of the WET period however, Figure 5.11f shows a very low inward-outward water flow from the storage compartments.

Figure $5.11 \mathrm{~g}$ in the DRY period shows the daily diametric oscillation of the trunk and how it steadily increases while reducing the oscillation range following the reduction in VPD. For the WET period, Figure $5.11 \mathrm{~h}$ shows a slight diametric oscillation of the trunk, but also how the xylem tissues are steadily growing. 
Finally, Figure 5.11i in the DRY period illustrates the status of the water potential of the considered compartments and how their variation inversely followed the VPD patterns. In the case of the WET period, Figure $5.11 \mathrm{j}$ illustrates the same water potential in response to VPD inverse pattern, as in the DRY period, but much less pronounced.

From comparing the patterns resulting in the DRY-period versus the WET-period simulation it is possible to conclude that the lower hydraulic resistance during WET period, reduced the effect of the VPD on the hydraulic status and the water dynamics of the tree. Therefore, the model helps us to understand how field-observed phenomena, such as the diameter variation of the trunk might be closely related to VPD dynamics, stomata closure, and the water potential operating in the system. Such a conclusion is justified because during drier environmental conditions trees would depend on stored water to cope with water-demand for physiological activities, such as photosynthesis and growth, thus causing more apparent circular variations.

\subsection{Conclusions}

Integrating water sourcing and sap flow measurements within a modelling framework has provided interesting insights that increase understanding of the mechanism controlling the water abstraction by tree transpiration, as follows:

Following deuterium tracing experiments, the contribution of the groundwater source to transpiration of Q.i. and Q.p. was the highest $(\approx$ $90 \%$ ) at the beginning of the summer, when: (a) sunny days are longer, (b) humid conditions prevail in the soil and (c) the water table is $\approx 1 \mathrm{~m}$ deep. Towards the end of the summer, when water table moves down and soil moisture decreases, contributions of groundwater source decrease to $30 \%$ for Q.p. and $40 \%$ for Q.i. on average.

During the DRY period, the soil matric potential (water availability control) and inner tissue resistances (water pressure control) are the main controlling forces dictating from which compartments water is abstracted and transported from the soil to the canopy for Q.i. and Q.p. During this period the model represented average water-sources contributions appropriately. In the WET period, however, the water table was so shallow that it was the main factor driving water abstraction, and therefore the tree water flow model simulations were not able to explain the $90 \%$ abstraction found by the isotopic measurements.

Finally, transpiration was demand driven and not water availabilitydriven, thus only the contribution of sources were changing. In this process storage played a crucial role in maintaining transpiration rates at the peak of the dry season, and a high proportion of this stored water during the summer originated from the groundwater. 

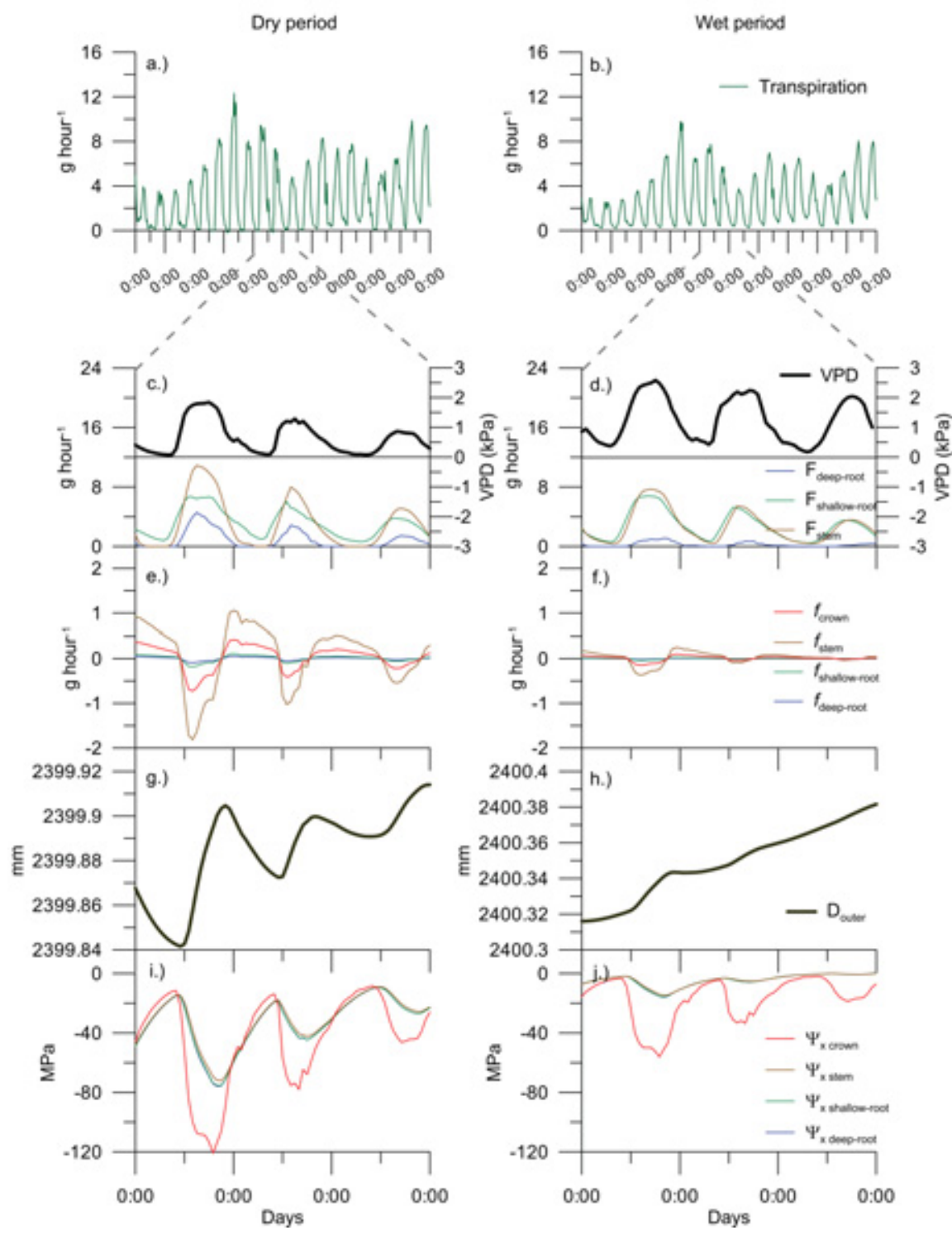

Figure 5.11: This figure shows the simulations run for Q.i. in the DRY (left) and WET (right) period. a) and b) show the stem flow simulations. c) and d) show three-day zoomed-in view of the stem flow (coloured lines) and VPD (black line). e) and f) show the storage water flows in (positive) and out (negative) of the storage compartments. g) and h) show the diameter variation of the trunk. i) and j) show the hourly variation of the water potential $(\Psi)$ defining the forces driving the water flow and diameter variation dynamics in the considered compartments. 


\subsection{Appendix}

\subsubsection{Roots-to-canopy water flow equations}

In this subsection the model is broken down into its constitutive equations focusing on the main functional elements of the model. At the beginning, the main water flows will be covered, followed by the mathematical representation of the capacity of the considered storage compartments. Afterwards, water potentials in the xylem and in the storage compartments are defined, together with the water flow from the storage compartment. The circular variability submodel is described in the next subsection. This arrangement does not represent the order of computations, but it allows the functioning of each section of the model to be more easily understood.

The flow rate in the xylem (i.e. vertical water transport) was defined with specific formulations for each compartment, some simpler than others to reduce complexity but all of them governed by Kirchoff's electrical current law. This law, when referring to tree cases, states that water flowing inside the tree might be distributed in different ways but it is equal to the water flowing out of the tree.

First, the main water flow out of the tree (i.e. transpiration $(T)$ ) was defined following Eq. 5.5

$$
T=\frac{\left(\Psi_{\text {air }}-\Psi_{\text {crown }}^{\mathrm{x}}\right)}{R_{\text {crown-air }}^{x}} \times-1
$$

where $\Psi_{\text {air }}$ is the water potential in the air above the canopy, $\Psi_{\text {crown }}^{\mathrm{x}}$ is the water potential in the xylem compartment of the tree crown and $R_{\mathrm{X} \text { crown-air }}$ is the resistance that needs to be overcome in the air spaces of the open stomata in the leaves.

The vertical xylem flow from the stem to the crown $\left(F_{\text {crown }}\right.$ in Figure 5.4) was defined as the interaction between transpiration $(T)$ and the flow $\left(f_{\text {crown }}\right)$ going in and out of the crown storage compartment, as in Eq. 5.6.

$$
F_{\text {crown }}=T \pm f_{\text {crown }}
$$

The vertical xylem flow from the roots to the stem $\left(F_{\text {stem }}\right.$ in Figure 5.4$)$ was defined as the interaction between the flow of water in the xylem in the crown xylem compartment and the flow going in and out of the stem storage compartment, as in Eq. 5.7.

$$
F_{\text {stem }}=F_{\text {crown }} \pm f_{\text {stem }}
$$


The flow path between the unsaturated zone and xylem of roots in the unsaturated zone ( $F_{\text {root unsat }}$ ) was defined following the electrical analogue (Figure 5.4) as in Eq. 5.8.

$$
F_{\text {root unsat }}=-\frac{\left(\Psi_{\text {root unsat }}^{\mathrm{x}}-\Psi_{\text {soil }}^{\mathrm{x}}\right)}{R_{\text {soil-root unsat }}^{x}} \pm f_{\text {root unsat }}
$$

where $\Psi_{\text {root unsat }}^{\mathrm{x}}$ is the water potential inside the roots at the unsaturated zone and $\Psi_{\text {soil }}^{\mathrm{x}}$ is the water potential of the surrounding soil. $R_{\text {soil-root unsat }}^{x}$ is the resistance in the root tissue between the soil water and the xylem in the roots that needs to be overcome for the water to flow inside. Finally, $f_{\text {root unsat }}$ represents the water flowing in and out from the storage compartment.

Table 5.4: Symbol, unit and description of the model variables. Pools (pool) are defined as crown, stem, roots in the unsaturated zone and roots in the saturated zone.

\begin{tabular}{|c|c|c|c|}
\hline Symbol & Unit & Description & Source \\
\hline$T$ & $\mathrm{~g} \mathrm{~h}^{-1}$ & Transpired water & Modelled \\
\hline$F_{\text {pool }}$ & $\mathrm{g} \mathrm{h}^{-1}$ & $\begin{array}{l}\text { Water flow paths between two dif- } \\
\text { ferent xylem compartments (e.g } \\
\text { flow path from stem to crown) }\end{array}$ & Modelled \\
\hline$f_{\text {pool }}$ & $\mathrm{g} \mathrm{h}^{-1}$ & $\begin{array}{l}\text { Water flow between xylem and stor- } \\
\text { age compartments in a given pool } \\
\text { (soil, stem, roots, etc.) }\end{array}$ & Optimised \\
\hline$W_{\text {pool }}$ & $g$ & $\begin{array}{l}\text { Water content in storage compart- } \\
\text { ment (pool) }\end{array}$ & $\begin{array}{l}\text { Initial value estimated, } \\
\text { then modelled }\end{array}$ \\
\hline$\Psi_{\text {pool }}^{x}$ & $\mathrm{MPa}$ & $\begin{array}{l}\text { Total water potential in a xylem } \\
\text { compartment }\end{array}$ & $\begin{array}{l}\text { Initial values optim- } \\
\text { ised, then modelled }\end{array}$ \\
\hline$\Psi_{\text {pool }}^{S}$ & $\mathrm{MPa}$ & $\begin{array}{l}\text { Total water potential in a storage } \\
\text { compartment }\end{array}$ & $\begin{array}{l}\text { Initial value optim- } \\
\text { ised, then modelled }\end{array}$ \\
\hline$\Psi_{\text {air }}$ & Mpa & $\begin{array}{l}\text { Water potential in the air above the } \\
\text { canopy }\end{array}$ & Modelled \\
\hline$\Psi_{\text {pool }}^{S}$ & Mpa & $\begin{array}{l}\text { Pressure component of the water } \\
\text { potential in storage compartment }\end{array}$ & $\begin{array}{l}\text { Initial value optim- } \\
\text { ised, then modelled }\end{array}$ \\
\hline$R_{\text {pool }}^{\mathrm{S}}$ & Mpa h $\mathrm{g}^{-1}$ & $\begin{array}{l}\text { Flow resistance between xylem and } \\
\text { a storage compartment }\end{array}$ & Optimised \\
\hline$R_{\text {pool }}^{\mathrm{X}}$ & Mpa $\mathrm{h} \mathrm{g}^{-1}$ & $\begin{array}{l}\text { Flow resistance in a xylem compart- } \\
\text { ment }\end{array}$ & Optimised \\
\hline$d^{S}$ & $\mathrm{~m}$ & $\begin{array}{l}\text { Thickness of the storage compart- } \\
\text { ment }\end{array}$ & Measured \\
\hline$D$ & $\mathrm{~m}$ & Outer diameter of stem storage & Measured \\
\hline$D_{i}$ & $\mathrm{~m}$ & Inner diameter of stem storage & Estimated \\
\hline$V^{s}$ & $\mathrm{~m}^{3}$ & Volume of storage compartment & Estimated \\
\hline$A$ & $\mathrm{~m}^{2}$ & $\begin{array}{l}\text { Surface area of the virtual mem- } \\
\text { brane separating the stem storage } \\
\text { compartment from the xylem com- } \\
\text { partment }\end{array}$ & Estimated \\
\hline
\end{tabular}

The flow path between the saturated zone and xylem of the deep roots ( $\left.F_{\text {root sat }}\right)$ was defined as the difference between the total flow to the stem $\left(F_{\text {stem }}\right)$, the flow from the unsaturated zone $\left(F_{\text {root unsat }}\right)$, and the 
Table 5.5: Symbol, unit and description of the model parameters. Pools are defined as crown, stem, roots in the unsaturated zone and roots in the saturated zone.

\begin{tabular}{|c|c|c|c|c|}
\hline Symbol & Unit & Description & Source & \\
\hline$p_{\text {pool }}$ & unitless & $\begin{array}{l}\text { Proportional representation of the } \\
\text { volume of a specified pool, relative } \\
\text { to the crown volume }\end{array}$ & Optimised & \\
\hline$a$ & $\mathrm{~m}$ & Allometric parameter & $\begin{array}{l}\text { Steppe and } \\
(2007 b)\end{array}$ & Lemeur \\
\hline$b$ & $\mathrm{~m}^{-1}$ & Allometric parameter & $\begin{array}{l}\text { Steppe and } \\
(2007 \mathrm{~b})\end{array}$ & Lemeur \\
\hline$C_{\text {pool }}$ & $\mathrm{g} \mathrm{MPa}^{-1}$ & $\begin{array}{l}\text { Capacitance of storage compart- } \\
\text { ment of a pool }\end{array}$ & Optimised & \\
\hline$k_{1}($ pool $)$ & $\mathrm{g}$ & $\begin{array}{l}\text { Amount of stored water at the in- } \\
\text { flection point of the desorption } \\
\text { curve }\end{array}$ & $\begin{array}{l}\text { Steppe and } \\
(2007 \mathrm{~b})\end{array}$ & Lemeur \\
\hline$k_{2}(\mathrm{pool})$ & unitless & $\begin{array}{l}\text { Index for rate of change of } \Psi_{\text {pool }}^{S} \text { at } \\
\text { the inflection point of the desorp- } \\
\text { tion curve }\end{array}$ & $\begin{array}{l}\text { Steppe and } \\
(2007 \mathrm{~b})\end{array}$ & Lemeur \\
\hline$l$ & $\mathrm{~m}$ & Length of stem segment & Measured & \\
\hline$L$ & $\mathrm{~m} \mathrm{MPa}^{-1} \mathrm{~h}^{-1}$ & $\begin{array}{l}\text { Radial hydraulic conductivity of the } \\
\text { virtual membrane separating the } \\
\text { stem storage compartment from } \\
\text { the xylem compartment }\end{array}$ & $\begin{array}{l}\text { Steppe and } \\
(2007 \mathrm{~b})\end{array}$ & Lemeur \\
\hline$\Phi$ & unitless & $\begin{array}{l}\text { Empirical parameter for the initial } \\
\text { condition of the pressure potential } \\
\text { of the storage compartment }\left(\Phi_{p s}\right)\end{array}$ & $\begin{array}{l}\text { Steppe and } \\
(2007 \mathrm{~b})\end{array}$ & Lemeur \\
\hline$\varepsilon$ & $\mathrm{MPa}$ & $\begin{array}{l}\text { Bulk elastic modulus of living tissue } \\
\text { in relation to reversible dimensional } \\
\text { changes (water storage) }\end{array}$ & $\begin{array}{l}\text { Steppe and } \\
(2007 \mathrm{~b})\end{array}$ & Lemeur \\
\hline$\varepsilon_{0}$ & $\mathrm{~m}^{-1}$ & Proportionality constant & $\begin{array}{l}\text { Steppe and } \\
(2007 \mathrm{~b})\end{array}$ & Lemeur \\
\hline$\rho_{\mathrm{W}}$ & $\mathrm{g} \mathrm{m}^{-3}$ & Density of water & $\begin{array}{l}\text { Steppe and } \\
\text { (2007b) }\end{array}$ & Lemeur \\
\hline$\Gamma$ & & $\begin{array}{l}\text { Critical value for the pressure com- } \\
\text { ponent }\left(\Psi_{S-\text { stem-p }}\right) \text { which must be ex- } \\
\text { ceeded to produce (positive) growth } \\
\text { in the storage compartment }\end{array}$ & $\begin{array}{l}\text { Steppe and } \\
(2007 \mathrm{~b})\end{array}$ & Lemeur \\
\hline
\end{tabular}

water flowing in and out of the storage compartment in the saturated zone ( $\left.f_{\text {root sat }}\right)$ as in Eq. 5.9.

$$
F_{\text {root sat }}=F_{\text {stem }}-F_{\text {root unsat }} \pm f_{\text {root sat }}
$$

In addition to vertical water transport $(F)$, stored water can contribute to daily transpiration due to flow $(f)$ resulting from the hydraulic connection between xylem and the storage pools (Simonneau et al., 1993; Zweifel et al., 2000, 2001; Zweifel and Häsler, 2001; Génard et al., 2001; Steppe et al., 2004; Steppe and Lemeur, 2004). Such contribution is dependent on the magnitude of the hydraulic exchange resistance $\left(R^{\mathrm{s}}\right)$ that must be overcome when water flows from the storage compartments toward the xylem to equilibrate the imbalance caused by the water demand during 
the day (Lhomme et al., 2001). The water flow to or from the storage pools $\left(f_{\text {pool }}\right)$ in the same compartment (e.g. within the stem) can then be defined as:

$$
f_{\text {pool }}=\frac{\Psi_{\text {pool }}^{x}-\Psi_{\text {pool }}^{s}}{R^{s}}
$$

where $\Psi_{\text {pool }}^{x}$ is the water potential in the xylem of the compartment, $\Psi_{\text {pool }}^{S}$ is the water potential in the storage section of the compartment, and finally $R^{\mathrm{s}}$ is the resistance inside the storage compartment that needs to be overcome for the water to flow in and out of the storage compartment.

Values of $f_{\text {pool }}$ are negative when water is abstracted from the storage pools and positive when the storage pools are refilled. Eq. 5.10 was used to define water flowing in and out of storage pools.

Regarding the pool compartments, originally the electrical analogue approach (see Figure 5.4) by Steppe et al. (2006) used a function to relate the water content to the total water potential, capacitance and the maximum capacity of the storage compartment. In the case of this study most storage compartments were defined following the original formulation of Steppe et al. (2006), with the exception of the crown pool $\left(W_{\text {crown }}\right) . W_{\text {crown }}$ was empirically defined for simplicity as a proportion of the stem pool $\left(W_{\text {stem }}\right)$ as in Eq. 5.11.

$$
W_{\text {crown }}=p_{\text {crown }} \times W_{\text {stem }}
$$

where $p_{\text {crown }}$ is a parameter representing the proportional relation between $W_{\text {crown }}$ and $W_{\text {stem }}$ (i.e. $\left.\frac{W_{\text {crown }}}{W_{\text {stem }}}\right)$.

The stem pool $\left(W_{\text {stem }}\right)$, following the electrical analogue, was defined as Eq. 5.12.

$$
W_{\text {stem }}=\left(\Psi_{\text {stem }}^{s} \times C_{\text {stem }}\right)+W_{\text {stem max }}
$$

where $\Psi_{\text {stem }}^{x}$ is the water potential in the xylem, $C_{\text {stem }}$ is the capacitance in the compartment, and $W_{\text {stem max }}$ is the maximum capacity of the stem compartment. This equation was derived by solving the differential Eq. 14 in Steppe et al. (2006) for $W_{\text {stem, }}$, where the capacitance is considered as non-variable for the electric analogue.

The root storage compartments for the saturated and unsaturated zones ( $W_{\text {root sat }}$ and $W_{\text {root unsat }}$, respectively) were defined following the electrical analogue and by combining Eqs. 3,6, and 14 in Steppe et al. (2006). By combining these equations the necessary extra terms to calculate the unknown water potentials ( $\Psi_{\text {root sat }}^{s}$ and $\left.\Psi_{\text {root unsat }}^{S}\right)$ were derived as in Eqs. 5.13 and 5.14:

$$
\begin{array}{r}
W_{\text {root sat }}=\left[\left(\Psi_{\text {root sat }}^{\chi}-F_{\text {stem }}\right) \times R_{\text {root sat-stem }}^{x} \times C_{\text {root sat }}\right] \\
+W_{\text {root sat-max }}
\end{array}
$$




$$
\begin{array}{r}
W_{\text {root unsat }}=\left[\left(\Psi_{\text {root unsat }}^{x}-F_{\text {stem }}\right) \times R_{\text {root unsat-stem }}^{x} \times C_{\text {root }}\right] \\
+W_{\text {root usat-max }}
\end{array}
$$

From the relation between the changes in water storage flow and capacitance (Eq. 14 in Steppe et al. (2006)) the water potentials in the storage compartments were derived as shown in Eqs. 5.15 to 5.18:

$$
\begin{aligned}
& \Psi_{\text {crown }}^{s}=\frac{\left(W_{\text {crown }}-W_{\text {crown-max }}\right)}{C_{\text {crown }}} \\
& \Psi_{\text {stem }}^{s}=\frac{\left(W_{\text {stem }}-W_{\text {stem-max }}\right)}{C_{\text {stem }}} \\
& \Psi_{\text {root }}^{s}=\frac{\left(W_{\text {root unsat }}-W_{\text {root unsat-max }}\right)}{C_{\text {root unsat }}} \\
& \Psi_{\text {root sat }}^{s}=\frac{\left(W_{\text {root sat }}-W_{\text {root sat-max }}\right)}{C_{\text {root sat }}}
\end{aligned}
$$

The xylem water potentials across tree compartments are interconnected and follow the principle of mass balance. In the crown compartment, the water potential in the xylem ( $\left.\Psi_{\text {crown }}^{x}\right)$ was defined by solving Eq. 1 in Steppe et al. (2006), following Ohm's law, as follows (Eq. 5.19):

$$
\Psi_{\text {crown }}^{\chi}=\Psi_{\text {stem }}^{\chi}-\left(F_{\text {crown }} \times R_{\text {stem-crown }}^{x}\right)
$$

However, as at this stage $F_{\text {crown }}$ is not known, it was replaced by its equivalents from Eq. 6 in Steppe et al. (2006) as follows (Eq. 5.20):

$$
\Psi_{\text {crown }}^{x}=\Psi_{\text {stem }}^{x}-\left[\left(F_{\text {stem }}-f_{\text {stem }}\right) \times R_{\text {stem-crown }}^{x}\right]
$$

The xylem water potential in the saturated and unsaturated compartments ( $\Psi_{\text {root sat }}^{X}$ and $\Psi_{\text {root unsat }}^{X}$ ), here represented as Eqs.5.21 and 5.22, follows in general the same logic as Eq. 5.19, but instead of being subtracted the effect of the water movement between compartments is added to the water potential of the stem because water potential increases with depth (i.e. when the direction is from low water potential compartment to high water potential compartment).

$$
\Psi_{\text {root sat }}^{\chi}=\Psi_{\text {stem }}^{\chi}+\left(F_{\text {root sat }} \times R_{\text {root sat-stem }}^{x}\right)
$$




$$
\Psi_{\text {root unsat }}^{x}=\Psi_{\text {stem }}^{x}+\left(F_{\text {root unsat }} \times R_{\text {root unsat-stem }}^{x}\right)
$$

The xylem water potential in the stem compartment $\left(\Psi_{\text {stem }}^{x}\right)$ follows the same logic as Eq. 5.19 (high water potential compartment to low water potential compartment). Thus, as the stem connects the xylem of the roots of both the saturated and the unsaturated zone, the equation to calculate $\Psi_{\text {stem }}^{x}$ must account for the interaction of these compartments as shown in Eq. 5.23.

$$
\begin{array}{r}
\Psi_{\text {stem }}^{\chi}=\Psi_{\text {root sat }}^{x}-\left[\left(F_{\text {root sat }}-f_{\text {root sat }}\right) \times R_{\text {stem-crown }}^{x}\right]+ \\
\Psi_{\text {root unsat }}^{x}-\left[\left(F_{\text {root unsat }}-f_{\text {root unsat }}\right) \times R_{\text {stem-crown }}^{x}\right]
\end{array}
$$

However, as $\Psi_{\text {stem }}^{x}$ is needed to calculate both $\Psi_{\text {root unsat }}^{x}$ and $\Psi_{\text {root sat }}^{x}$ beforehand, both xylem water potentials were defined indirectly from another set of equations as follows.

First, from Eq. 5.10 both $\Psi_{\text {root sat }}^{x}$ and $\Psi_{\text {root unsat }}^{x}$ were isolated as shown in Eq. 5.24:

$$
\Psi_{\text {pool }}^{x}=\Psi_{\text {pool }}^{s}+\left(f_{\text {pool }} \times R_{\text {pool }}^{s}\right)
$$

Then, assuming that $f_{\text {root sat }}$ and $f_{\text {root unsat }}$ can be explained as a proportions of $f_{\text {stem }}$ we derive Eq. 5.25 and Eq. 5.26.

$$
\begin{aligned}
& f_{\text {root sat }}=p_{\text {root sat }} \times f_{\text {stem }} \\
& f_{\text {root unsat }}=p_{\text {root unsat }} \times f_{\text {stem }}
\end{aligned}
$$

By combining Eq. 5.23 with Eq. 5.24, Eq. 5.25 and Eq. 5.26, the equation to calculate $\Psi_{\text {stem }}^{x}$ (Eq. 5.27) is finally obtained.

$$
\begin{array}{r}
\Psi_{\text {stem }}^{x}=\frac{R_{\text {stem }}^{s}-\left(R_{\text {sat }}^{s} \times p_{\text {root sat }}\right)}{R_{\text {stem }}^{s}} \times \\
{\left[\Psi_{\text {sat }}^{s}-\left(\frac{\Psi_{\text {stem }}^{s} \times R_{\text {sat }}^{s} \times p_{\text {root sat }}}{R_{\text {stem }}^{s}}\right)-\right.} \\
\left.\left(F_{\text {stem }} \times R_{\text {stem-sat }}^{x}\right)\right]+ \\
\frac{R_{\text {stem }}^{s}-\left(R_{\text {unsat }}^{s} \times p_{\text {root unsat }}\right)}{R_{\text {stem }}^{S}} \times \\
\Psi_{\text {unsat }}^{s}-\left(\frac{\Psi_{\text {stem }}^{s} \times R_{\text {sunsat }}^{s} \times p_{\text {root unsat }}}{R_{\text {stem }}}\right)- \\
\left.\left(F_{\text {stem }} \times R_{\text {root unsat-stem }}^{x}\right)\right]
\end{array}
$$


The last set of variables $f_{\text {crown }}$ and $f_{\text {stem }}$ were derived as follows:

$$
f_{\text {crown }}=p_{\text {crown }} \times f_{\text {stem }}
$$

$f_{\text {crown }}$ was also calculated as a proportion of $f_{\text {stem, }}$, where $p_{\text {crown }}$ corresponds to an optimised parameter representing a proportion of water moving in and out of the storage in the crown relative to the amount of water moving from the storage in the stem. The assumption here is that water movement in the storage compartment at the crown and stem differ only in magnitude and not in direction. The assumption is sensible because these compartments are not independent, and because crown storage water flow is low.

The storage flow in the stem $\left(f_{\text {stem }}\right)$ was estimated as in Eq. 5.29 , by substituting $\Psi_{\text {stem }}^{x}$ with Eq. 5.27.

$$
f_{\text {stem }}=\left(\Psi_{\text {stem }}^{x}-\Psi_{\text {stem }}^{s}\right) / R_{\text {stem }}^{s}
$$

Finally, water potential from the air was calculated as described by Lambers et al. (1998), using the climatic variables air temperature ( $\left.T_{\text {air }}\right)$, relative humidity $(R H)$ and the molar volume of water $\left(V w_{0}\right)$, as follows.

$$
\Psi_{\text {air }}=\left[\frac{R \times\left(T_{\text {air }}+273.15\right)}{V w_{0}}\right] \times \lg \left(\frac{R H}{100}\right)
$$




\subsubsection{Basic equations for the stem diameter variation submodel (SDVS)}

Steppe et al. (2006) used the stem geometry proposed by Génard et al. (2001) to define the equations used in the SDVS.

The thickness of the storage compartment $\left(d^{\mathrm{s}}\right)$ is equal to:

$$
d^{\mathrm{s}}=\frac{D_{\text {outer }}-D_{\text {inner }}}{2}
$$

where $D_{\text {outer }}$ in this study was considered as the outer diameter of the stem segment without including the hardwood (i.e. sapwood outer diameter), and $D_{\text {inner }}$ was considered as the hardwood diameter in the segment.

The volume of the storage compartment $\left(V^{s}\right)$ is defined by the following equation:

$$
V^{\mathrm{s}}=\frac{\pi \times l}{4} \times\left(D_{\text {outer }}^{2}-D_{\text {inner }}^{2}\right)
$$

where $l$ is the stem segment length. As $d^{\mathrm{s}}$ in all measured trees during the field campaign of this thesis was much smaller than $D_{\text {inner }}$ (see Chapter 3), the assumption made by Steppe et al. (2006) was followed to use the rewritten version of Eq. 5.32 as follows:

$$
V^{\mathrm{s}} \approx \pi \times d^{\mathrm{s}} \times D_{\text {inner }} \times l
$$

This equation is a simplified approach to reduce the complexity of the submodel and it incurs less than 10\% error (Steppe et al., 2006; Génard et al., 2001).

The amount of stem diameter variation depends on the bulk elastic modulus $(\varepsilon)$ that expresses the elasticity of cell walls, in other words, a larger elasticity of cell walls is defined by a smaller value of the elastic modulus (Lambers et al., 1998; Nobel, 1999) and was defined as:

$$
\varepsilon=\varepsilon_{0} \times D_{\text {outer }} \times \Psi_{\text {stem-p }}^{\mathrm{s}}
$$

where $\varepsilon$ is proportional to the initial elastic modulus $\left(\varepsilon_{0}\right)$ and to the product of the outer diameter $(D)$ and the pressure potential $\left(\Psi_{\mathrm{p}}^{\mathrm{s}}\right)$.

The variation in the stem $\left(d^{\mathrm{s}}\right)$ results from elastic changes driven by growth of the stem and the water potential in the storage compartment. These processes were defined by a system of differential equations (Eqs. 23 to 29 in Steppe et al. (2006)) from which the following final equations were used for the submodel.

$d^{\mathrm{s}}$ was defined as:

$$
\frac{\mathrm{d} d^{\mathrm{s}}}{d t}=a \times b \times e^{\left(-b \times D_{\text {outer }}\right)} \times \frac{d D_{\text {outer }}}{d t}
$$


where $a$ and $b$ are empirical parameters defining the relation between $D_{\text {inner }}$ and $D_{\text {outer }}$ (Génard et al., 2001), obtained from the observation that the thickness of extensible tissues increases with the diameter of the organ (Huguet, 1985).

$D_{\text {inner }}$ was defined as:

$$
\frac{d D_{\text {inner }}}{d t}=\frac{d D_{\text {outer }}}{d t}-2.0 \times \frac{d S}{d t}
$$

and finally $D_{\text {outer }}$ was defined from both the elastic changes and the growing status of the stem.

The growing component of the model is controlled by a pressure potential critical value $(\Gamma)$ set as a threshold to indicate which of the following equations to use for the calculation at each time-step:

This equation is used when $\Psi_{\text {stem-p }}^{\mathrm{s}}>\Gamma$

$$
\begin{array}{r}
\left(\frac{D_{\text {outer }}}{d t}\right)=2.0 \times d^{\mathrm{s}} \times \\
\left(\frac{\frac{d \Psi_{\text {stem-p }}^{\mathrm{s}}}{d t}}{\left(\varepsilon_{0} \times \Psi_{\text {stem-p }}^{\mathrm{s}} \times D_{\text {outer }}\right)}\right)+ \\
\left(d^{\mathrm{s}} \times \Phi \times \frac{\left(\Psi_{\text {stem-p }}^{\mathrm{s}}-\Gamma\right)}{\left(b \times\left(a-d^{\mathrm{s}}\right)\right)}\right)
\end{array}
$$

This equation is used when $\Psi_{\text {stem-p }}^{\mathrm{s}} \leq \Gamma$

$$
\begin{array}{r}
\left(\frac{D_{\text {outer }}}{d t}\right)=2.0 \times d^{\mathrm{s}} \times \\
\left(\frac{\frac{d \Psi_{\text {stem-p }}^{\mathrm{s}}}{d t}}{\left(\varepsilon_{0} \times \Psi_{\text {stem-p }}^{\mathrm{s}} \times D_{\text {outer }}\right)}\right)
\end{array}
$$

where $\varepsilon_{0}$ was calculated from Eq. 5.34 , and $\Phi$ is a proportionality constant defining the cell wall extensibility. $\Psi_{\text {stem-p }}^{\mathrm{s}}$ is an expression for the water pressure potential in the stem storage compartment to define the effect of growth in the SDVS (Steppe et al., 2006). The change in pressure potential $\Psi_{\text {stem-p }}^{\mathrm{s}}$ results from the flow of water in and out of the stem storage compartment and was defined as:

$$
\frac{d \Psi_{\text {stem-p }}^{\mathrm{S}}}{d t}=\frac{d W_{\text {stem }}}{d t} \times \frac{\varepsilon}{\rho_{\mathrm{w}} \times V_{\text {stem }}}
$$

Finally the exchange resistance $\left(R_{\text {stem }}^{\mathrm{s}}\right)$ is a result of the flow of water between xylem and storage compartment through a "virtual" membrane (A). $R_{\text {stem }}^{\mathrm{S}}$ is defined by Steppe et al. (2006) as inversely proportional to the relation between hydraulic conductivity $(L)$, the virtual membrane $(A)$ and the density of water $\left(\rho_{\mathrm{w}}\right)$, as in the following equation.

$$
R_{\mathrm{S} \text {-stem }}=\frac{1}{\left(A \times \rho_{\mathrm{W}} \times L\right)}
$$


The "virtual" membrane $A$ is defined as a "single" cell model, and represents several layers of tissue that lead to symplastic or apoplastic water flow, or both, and is defined as:

$$
A=\pi \times D_{\text {inner }} \times l
$$

Eq. 5.40 establishes the direct link between the water flow submodel and the SDVS, because $R_{S \text {-stem }}$ is used to calculate $f_{\text {stem }}$ in Eq. 5.29. 


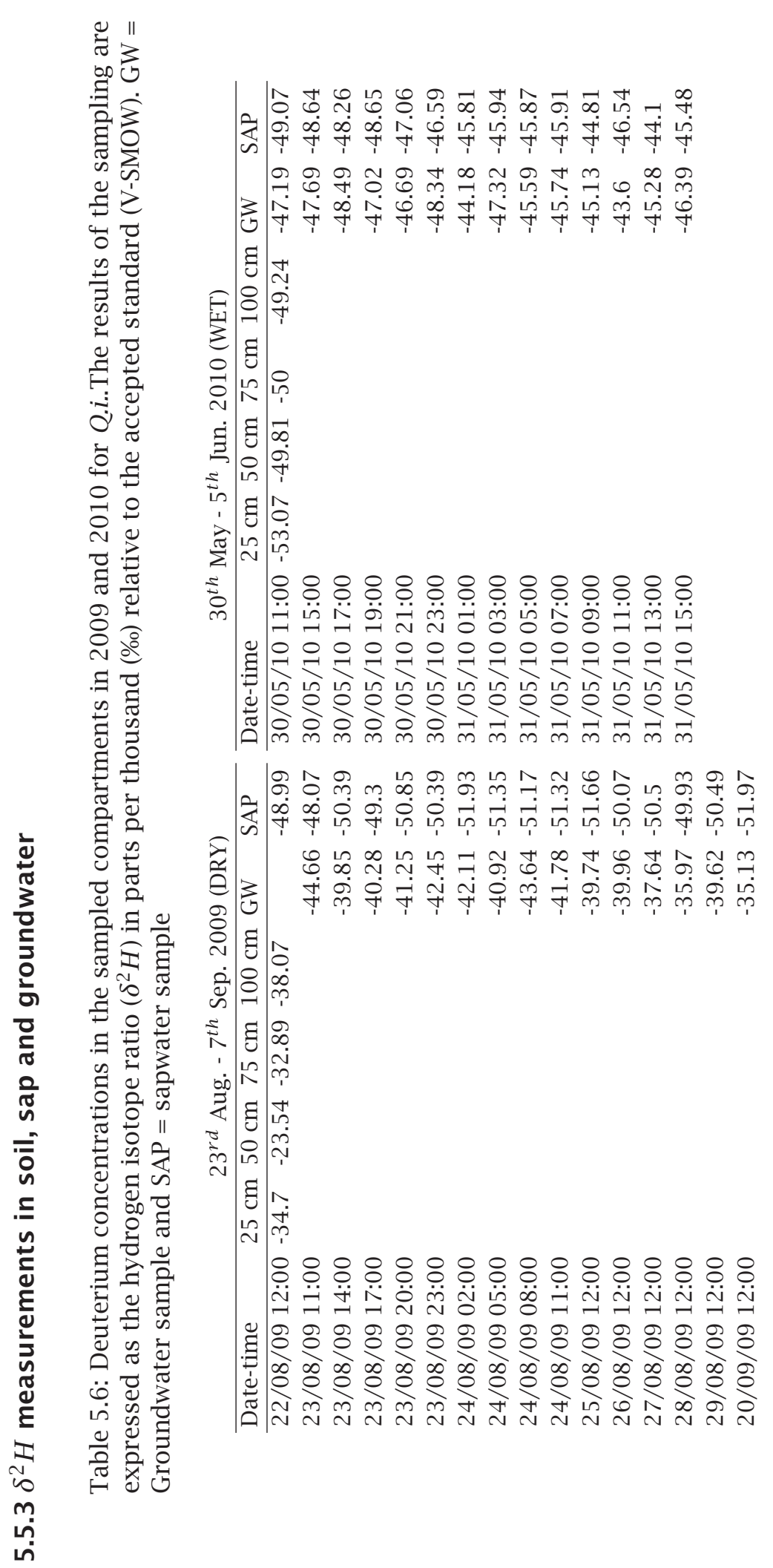




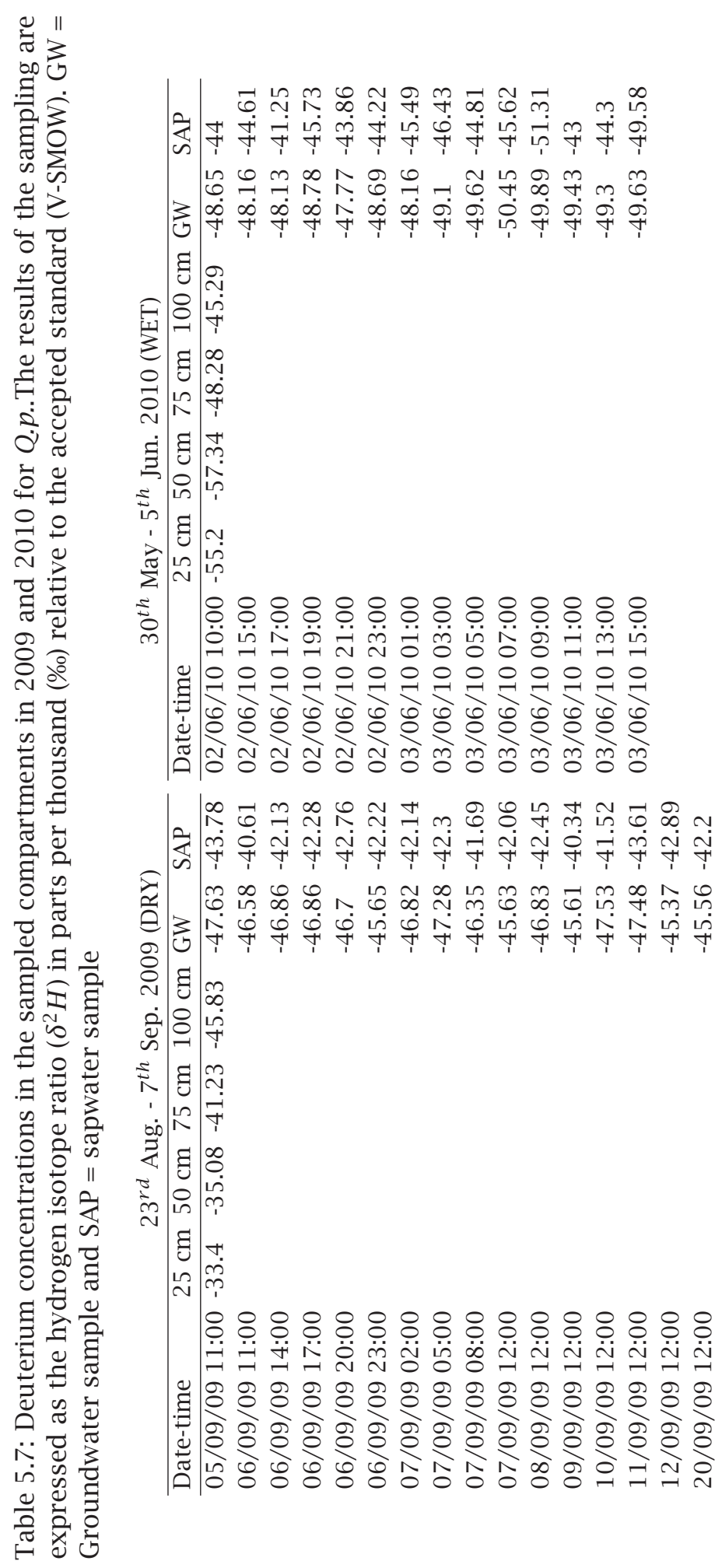




\section{Scaling up water-source contributions to tree-transpiration}

Figure 6.1: Patches of Quercus ilex and Quercus pyrenaica trees in the Sardón catchment during the dry season in September 2009.

As observed, grasses and herbaceous plants have all wilted away, indicating water deficit in the soil, but the foliage of oak trees displays a lush green colour. 


\begin{abstract}
Identifying and quantifying key components of the hydrological cycle such as evaporation $(E)$ and transpiration $(T)$ has been of the utmost priority in many studies, with a view to properly managing water resources. This study aimed to quantify (source) two components of $(T)$ tree transpiration, namely: groundwater transpiration $\left(T_{g}\right)$ and unsaturated zone transpiration $\left(T_{u}\right)$, at the forest stand and catchment scales for two oak species Quercus ilex (Q.i.) and Quercus pyrenaica (Q.p.). To achieve this two techniques were combined: (a) $T$ scaling-up using satellite imagery during the early dry-season (WET) and the late dry-season (DRY); and (b) water-source tracing using naturally-abundant stable isotopes.

It was found that Q.i. and Q.p. have developed different and contrasting strategies to confront the summer drought, and these are reflected in the spatio-temporal patterns of stand transpiration components $T_{\text {sta-g }}$ and $T_{\text {sta-u. }}$ Q.i. for example is located further from river banks and ephemeral rivers, but abstracts as much groundwater as Q.p., which is normally found in places with the shallowest groundwater levels. In addition, it was found that groundwater uptake decreases during the summer drought for both Q.i. and Q.p., but in overall groundwater is still the main water source for these oak species to survive.

Interestingly, tree transpiration at the catchment scale $\left(T_{c}\right)$ only slightly decreases from WET $\left(\approx 0.027 \mathrm{~mm} \mathrm{day}^{-1}\right)$ to DRY $(\approx 0.023$ $\mathrm{mm}$ day $^{-1}$ ). This indicates that both species seem to successfully cope with the very dry conditions dominant at the end of the summer, but also how dependent they are on groundwater sources to survive. If trees were not able to tap groundwater they would simply not exist in this area. These insights offer great potential for deriving appropriate water management strategies for Mediterranean areas with similar conditions.
\end{abstract}

\title{
6.1 Introduction
}

The discrimination of water sources such as, soil water, groundwater, mist, etc. has become an important focus of hydrological studies concerning various regions and hydrological conditions (David et al., 2007; Zencich et al., 2002; Bredemeier et al., 2011). This knowledge has proved valuable in increasing understanding of the mechanisms of vegetation water uptake, and the impact of such uptakes upon hydrological water compartments (see Chapter 5). Generally speaking sourcing such uptakes begins by understanding the characteristics of the hydrological cycle in the studied area and then defining the two main components of water loss in non-liquid form, namely: evaporation $(E)$ and tree transpiration (T). Depending on the scientific approach, and/or interest, then $E$ or $T$ are broken down into their constitutive parts (see Chapter 1 for a detailed explanation of the different components of the water balance).

Diverse studies have attempted to trace the movement of water sources through hydrological compartments (e.g. stem, unsaturated 
zone, roots, etc.) in the soil-plant-air continuum at different spatial scales. For example, Cavanaugh et al. (2011) studied the distribution of pulse-precipitation water components in shrubby vegetation in North American desserts and attempted to trace the different paths of pulsewater through the system; Rothfuss et al. (2010) simulated the conditions for a phreatophyte in a climatic chamber and attempted to partition the evapotranspiration (ET) fluxes; and Schwinning et al. (2002) studied the forms in which rain water in the soils is used by shrub/grass species in the Colorado Plateau using enriched isotopes.

Other studies, have focused more on quantifying the proportions in which water sources are distributed within $E$ or $T$, without considering their origin. They have achieved this by measuring actual $E T$ and then partitioning it using diverse techniques, such as isotopes-tracing (Dawson et al., 2002), hydrological modelling (Baird and Maddock, 2005), and sapflow (T) measuring techniques (Cavanaugh et al., 2011; David et al., 2004; Hernández-Santana et al., 2008c). Evaporation $E$ is estimated as the difference between $E T$ and $T$ (Lubczynski and Gurwin, 2005; Lubczynski, 2009; Yaseef et al., 2010; Rothfuss et al., 2010). ET quantifications are normally obtained from eddy-covariance (Yaseef et al., 2010) or Bowenratio (Brunel et al., 1997) measurements, amongst others.

In the last couple of years ET quantification using eddy covariance measurements from towers, which are usually performed at large foreststands of $\approx 100 \mathrm{ha}^{-1}$ (called simply stands in the rest of the chapter), has been widely used in many studies (Wilson et al., 2001; Williams et al., 2004; Rothfuss et al., 2010; Cavanaugh et al., 2011). Such studies describe interesting observations and dynamics but are inevitably limited to the erratic variability (spatial and temporal) of the foot-print areas of the eddy covariance towers (Finnigan, 2004; Göckede et al., 2004). Therefore, eddy covariance measurements in uneven terrains and irregular surfaces, such as patchy forests or shrubby-savannahs, might be of only limited use for properly discriminating the spatial-variation of hydrological components (Balugani et al., 2011).

This limitation is even more compelling if the aim is to understand the dynamics of finer components of the unsaturated such as $E_{u}$ and $T_{u}$ and and groundwater components such as $E g$ and $T_{g}$. Measuring, these finer components corresponds to an urgent need for better understanding of the hydrological systems to facilitate better water management especially critical for water limited environments (Lubczynski, 2009) particularly in the context of land use and climate-change.

A possibility, however, is to combine (1) ground sourcing measurements to identify the distribution of components at smaller scales (e.g. isotopes tracing at the tree level), with (2) satellite imagery scaling-up techniques to adequately extrapolate the measurements to the large scales.

The methodological approaches to apply this process for $T$ and $E$ are clearly different (Balugani et al., 2011; Lubczynski, 2009), as $T$ is focused on the vegetation and $E$ mostly on the soil. This study focuses only on $T$ scaled-up sap flow measurements for Quercus ilex (Q.i.) and Quercus 
pyrenaica (Q.p.) (see Chapter 3), and their sourced water contributions from the saturated and unsaturated zone of the subsurface (see Chapter $5)$.

Using such measurements, this study aimed at representing the spatial variation of the contributions to tree transpiration $(T)$ of the saturated $\left(T_{g}\right)$ and unsaturated $\left(T_{u}\right)$ zones at the (a) stand $\left(T_{\text {sta }}\right)$ and (b) catchment $\left(T_{\mathcal{C}}\right)$ scale as dependent on the Q.i. and Q.p. vegetation cover.

\subsection{Materials and methods}

\subsubsection{Calculating $T_{g}$ and $T_{u}$ at tree level}

The WET period represents the first part of the dry season, when the water compartments have been replenished due to spring rains and the water table is close to its highest yearly peak. In contrast, the DRY period represents the later stages of the dry season, when the soil has reached its driest point and most of the grasses and shallow plants have wilted due to water scarcity. During DRY, the water table reaches the lowest point in the year and ephemeral rivers have all dried up.

The calculation of $T_{g}$ and $T_{u}$ for the Sardón catchment was done by combining two separate data sets, one contributing the spatial dimension (Chapter 4) and the other the temporal dimension (Chapters 3 and 5). The first one corresponds to the maps with the locations and estimations of $T$ for each Q.i. and Q.p. tree early in the dry-season period, i.e. WET, (May to June 2010) and late in the dry-season period, i.e. DRY (August to September 2010). The second, is the data set of hourly water-source contributions to $T$ as obtained from sourcing discretisation.

The tree $T$ maps during the WET and DRY periods were the product of using satellite-imagery to define tree species and canopy cover characteristics, which were mathematically linked to sap flow measurements using a scaling-up method. The whole procedure can be found in Chapter 4.

Water-source contributions were estimated using techniques to trace naturally abundant isotopes at three-hourly intervals for 24 hours (See Chapter 5 for more details on the isotopes tracing, and the Appendix section 6.5 for a visualization of the isotopes/sap flow measurements). These measurements provided an opportunity to calculate water contributions with a good accuracy within a day, as water uptakes varies hourly and seasonally. Originally, it was planned to model the watercontribution measurements throughout the whole dry season to get continuous data coverage. Unfortunately, the selected water-plant model was not suitable for properly capturing the high water-table conditions during the WET period and thus daily estimates were not available to upscale $T_{g}$ and $T_{u}$. More information about these measurements can be found in Chapter 5 .

So in order to meet the objectives of this study, the following assumptions were necessary: (1) the isotopic contributions measured for 
each species were assumed to be representative of Q.i. and Q.p. trees at the catchment scale in WET and DRY, even-though these measurements were part of a limited sampling schema close to the valley where water table is shallow thus accessible with available drilling equipment. This assumption may incur in estimation errors because of shallower water table than catchment average in the location of sampling, but it is sensible because the selected trees were close to clusters of tree patches and had a size-range typically common of Q.i. and Q.p. trees $(30.5 \mathrm{~cm}$ and $34.8 \mathrm{~cm}$ respectively, see Table 3.2 in Chapter 3 for more information on the population structure of Q.i. and Q.p.). Additionally, as discussed in Chapter 4, the tree distribution across the catchment is linked to the proximity to water bodies where trees tend to cluster, more specifically, Q.p. clusters are the most common and occur as close as possible to river pathways where water table is the highest. Finally both species (particularly Q.i.) have deep root systems ( $>2 \mathrm{~m}$ deep) that would most likely be in direct contact with the water table.

Another assumption (2) is that dry-season variability of $T$ is low enough during the growing season to be statistically insignificant throughout most of the dry season (see the Appendix section 6.5, for sap flow measurements comparison during WET and DRY), for both species (although hourly variability is important for $Q . i$. as discussed bellow). This assumption is sensible, because sap flow measurement for standard clear-sky "Dry-season" conditions throughout the whole dry season (as shown in Section 5.3), had a low daily variability.

These assumptions allow us to scale our findings for any clear-sky day during the WET and DRY periods, which are the majority of days during dry season in the study area.

To derive $T$ maps with the water contributions, additional calculations were executed based on tree $T$ maps for the WET and DRY seasons. The additional calculations took into consideration the water contributions temporal variability, specific to each species. For $Q . i$. in the WET period $T_{g}$ was calculated as $95 \%$ and $T_{u}$ as $5 \%$ of total $T$ (as in Chapter 5). In the DRY period, however, due to the temporal variability found in the water contributions from the saturated zone to Q.i. (see Chapter 5), the daily calculations were done by discretising 24 hours into a number of periods of specific duration and sourcing percentages. This was done with the aim of harmonising as best as possible the peak of measured sap flow for Q.i. trees (see Chapter 3), with the peak of saturated zone uptake (see Chapter 5). This was necessary because peaks did not match, and thus a standard average would have overestimated or underestimated the sourcing quantities for $T_{g}$ and $T_{u}$

To discretise 24 hours of $T$ water contributions, daily periods were established to show the variability throughout the day, as seen in Table 6.1. The daily periods were divided as: (a) "Sunrise" (8 hours), (b) "Morning" (2 hours), (c) "Noon" (6 hours), (d) "Afternoon" (4 hours) and (e) "Sunset" (4 hours). These periods were defined by optimising the lowest variability possible at varying time intervals.

Once periods were defined and the average contributions calculated 
for each period, hourly sap flow values were discretised in the same way and then multiplied by the contributions. Then, the amount of water in all periods was added and expressed in area-units to calculate daily $T_{\mathscr{g}}$ and $T_{u}$.

In the case of Q.p. trees, the calculation was simpler than for Q.i., as the temporal variability of water contributions in the dry season for this species was low (see Subsection 5.3.3 in Chapter 5). $T_{g}$ was calculated for each cell in the catchment as the $63 \%$ of the total transpiration flow (T) for DRY, and as $98 \%$ for WET. $T_{u}$ was calculated as $37 \%$ for DRY and $2 \%$ for WET.

Table 6.1: Water source contributions used to account for temporal variability in the estimations of $T_{\text {sta }}$ and $T_{c}$.

\begin{tabular}{llllll}
\multicolumn{4}{c}{ WET } & \multicolumn{2}{c}{ DRY } \\
\hline & $T_{g}$ & $T_{u}$ & & $T_{g}$ & $T_{u}$ \\
\hline Q.i. & $95 \%$ & $5 \%$ & "Sunrise" & $35 \%$ & $65 \%$ \\
& & & "Morning" & $47 \%$ & $53 \%$ \\
& & & "Noon" & $61 \%$ & $39 \%$ \\
& & & "Afternoon" & $41 \%$ & $59 \%$ \\
& & & "Sunset" & $37 \%$ & $63 \%$ \\
\hline Q.p. & $98 \%$ & $2 \%$ & & $63 \%$ & $37 \%$ \\
\hline
\end{tabular}

\subsubsection{Scaling up $T_{g}$ and $T_{u}$ at the stand and catchment level}

After calculating daily $T_{g}$ and $T_{u}$ for each tree in the catchment during WET and DRY, $T_{g}$ and $T_{u}$ were scaled up to the whole catchment at 1 ha cell resolution, resulting in two partitioned elements, representing the stand level ( $T_{\text {sta-g }}$ and $\left.T_{\text {sta-u }}\right)$, and the whole at catchment level ( $T_{c-g}$ and $\left.T_{c-u}\right)$. This study focuses on catchment-level estimates only, and thus no specific stand was considered.

The scaling-up procedure is explained in detail in Chapter 4, but in general it follows three steps. First, a 1 ha grid is overlaid on the tree level $T_{g}$ and $T_{u}$ calculations. The grid groups and "slices" tree covers to make self-contained units. Second, the pieces of tree covers are added together and re-projected to 1 ha to calculate $T_{\text {sta-g }}$ and $T_{\text {sta-u }}$ for each species in WET and DRY to obtain the stand-scale maps. And finally, the stand-scale maps are averaged to calculate the catchment partitions $T_{c-g}$ and $T_{c-u}$.

All calculations were made using the "spatial calculator" in the ArcMAP software. 


\subsection{Results and discussion}

\subsubsection{Spatio-temporal patterns of $T_{\text {sta-g }}$ and $T_{\text {sta-u }}$ partitions}

To better appreciate the spatio-temporal temporal variations captured in the analysis, the results are organised by WET and DRY periods as in Chapter 5.

In Figure 6.2 the spatial distribution for $T_{\text {sta-g }}$ and $T_{\text {sta-u }}$ in the whole Sardón catchment can be observed for both WET and DRY. Both $T_{\text {sta-g }}$ and $T_{\text {sta-u }}$ follow the vegetation patches and clusters, which in turn follow the distribution of the main river courses across the catchment. The largest tree stand, where the highest $T_{\text {sta-g }}$ and $T_{\text {sta-u }}$ rates for both seasons are concentrated, is established in the north-easternmost part of the catchment (see Figure 6.2 and/or Figure 4.9). This is the lowest area in the catchment, where the catchment outlet is located and therefore water availability is greater than in the southernmost part where fewer trees are observed.

In terms of temporal variation there was slight but not significant decrease in overall catchment transpiration or $T_{c}$ (average of $T_{\text {sta }}$ ) from WET $\left(0.027 \mathrm{~mm} \mathrm{day}^{-1}\right)$ to DRY $\left(0.023 \mathrm{~mm}\right.$ day $\left.^{-1}\right)$. The slight decline can be related to different factors, such as: (a) water availability is lower throughout the region as compared with the beginning of the dry season: (b) days are shorter than at the beginning of the season, thus shortening the daily photosynthesis cycles: and (c) Q.p. trees in general start loosing leaves and the remainder ones are not as efficient as at the beginning of the season (Q.p. does not renew leaves after the first flush in spring).

Although this slight decrease was present, $T_{\mathcal{C}}$ remains fairly stable from WET to DRY. This was expected because sap flow measurements displayed a low daily variation throughout the whole summers season for clear-sky days (see Chapters 3 and 4), despite general lower water availability. David et al. (2007) have made similar observations regarding Q.i. in Portugal.

Nevertheless, significant temporal changes between WET and DRY periods occur in the proportion of water abstracted from the unsaturated $(u)$ and saturated $(g)$ zones of the subsurface. First, $T_{c-u}$ (average of $T_{\text {sta-u }}$ across the catchment) significantly increased in the DRY season $\left(0.02 \mathrm{~mm} \mathrm{day}^{-1}\right)$ as compared with the WET season $\left(<0.010 \mathrm{~mm} \mathrm{day}^{-1}\right)$. In contrast, $T_{c-g}$ (average of $T_{\text {sta-g }}$ across the catchment) in the DRY season $\left(0.025 \mathrm{~mm} \mathrm{day}^{-1}\right)$ decreased nearly twice from the rates measured during the WET season $\left(0.045 \mathrm{~mm} \mathrm{day}^{-1}\right)$. These temporal differences indicate a significant and fast shift in the sourced water contributions of abstracted water throughout the summer. This is a significant change during the season explained on the receding water table that gives way to an increasing abstraction from unsaturated layers. Such dynamic might be characteristic of areas with shallow groundwater similar to the one where trees were sampled in the Sardón catchment.

These results suggest that early in the dry season, $T_{\mathcal{C}}$ mostly originates from the saturated zone, but then gradually towards the end of the 
Table 6.2: Temporal variation of $T_{\text {sta-g }}, T_{\text {sta-u }}$, and $T_{\mathcal{C}}$ in the Sardón catchment

\begin{tabular}{lllll} 
Statistic & WET $T_{\text {sta-g }}$ & WET $T_{\text {sta-u }}$ & DRY $T_{\text {sta-g }}$ & DRY $T_{\text {sta-u }}$ \\
\hline Max. & 0.452 & 0.018 & 0.204 & 0.267 \\
Min. & $<0.010$ & $<0.010$ & $<0.010$ & $<0.010$ \\
Average $\left(T_{c}\right)$ & 0.045 & $<0.010$ & 0.025 & 0.021 \\
\hline * All measurements in mm day day $^{-1}$ & &
\end{tabular}

summer, the water table recedes and the vegetation is forced to abstract water from the unsaturated zone, thus causing a decrease in $T_{\text {sta-g }}$ and a significant increment in $T_{\text {sta-u }}$. These results are somewhat surprising, as findings such as those of Hultine et al. (2004), indicate that groundwater uptake increases when moisture in the unsaturated decreases. However, the sampled water table was so shallow that trees had access to it very early in the dry-season (i.e. during the WET period), as demonstrated by isotopic measurements (Chapter 5), and thus trees behave more like a riparian species.

Such dynamics have been observed in previous studies on riparian species. For example Baird and Maddock (2005) proposed a semiempirical approach to quantify and explain $E T$ flows in plant-groundwater interactions by representing uptakes of water sources as functions of water-table depth. They proposed that vegetation decreases $T$ rates either when the water table is so shallow that the tolerance to anoxic conditions in the soil is reached by the vegetation, or when the water table is so low that roots would not be able to abstract water from it. But all in all, they consider $T$ as a process of water-table availability or excess, which aligns with the findings in the Sardón catchment.

However, there are also differences between the findings of Baird and Maddock (2005) and the ones of this study. In their case $T$ changes as the water table recedes, whereas the findings of this study indicate that $T_{C}$ remains relatively constant throughout the season.

The investigated shallow water table conditions in the Sardón catchment and the unique traits of Mediterranean oak trees, are responsible for this small difference. Basically, Q.i. and Q.p. trees have a continuous source of water. In the beginning of the dry-season most of the water gets abstracted from the shallow groundwater; then gradually more of it gets abstracted from the unsaturated zone as the water table recedes; and finally at the end of the dry-season water uptake is divided more or less equally between the two sources.

These dynamics suggest that Q.i. and Q.p. have a considerable tolerance to anoxic soil conditions (i.e. soil saturated with water), and at the same time their root depths are such that trees can tap into the water even at its lowest point (5 metres on average).

Therefore, if designing a plausible extinction curves for Q.i. and Q.p. following Baird and Maddock (2005), it would be almost like a straight 
line with a slight inclination when reaching maximum water table depth limits (i.e. like an exponential distribution), with a different inclination for each species. Such a curve would work for most modelling initiatives, such as MODFLOW (see Harbaugh et al., 2000), and it would be very different from the the typical riparian species curve described by Baird and Maddock (2005).

Nevertheless, to better represent the dynamics described in this study for Q.i. and Q.p. in hydrological models (e.g. MODFLOW, SWAT, etc.), it is suggested that future applications split the extinction curves into two components. The first component would represent the proportion abstracted from the unsaturated zone $\left(T_{u}\right)$ and how it changes throughout the season, and the second component would represent the saturated zone $\left(T_{g}\right)$. Thus the shallower the water table, the higher the contribution from the saturated zone and vice-versa. The use of such curves enables uptake flows from these compartments to be taken into account and ultimately accuracy can be improved (see Francés et al. (2011) for an example of an application of this kind).

In conclusion, $T_{\mathcal{C}}$ is mostly related to the proportionality of water source contributions and as the water table is shallow in Sardón, it is thus maintained at almost the same rate throughout the whole year. Moreover, the spatio-temporal patterns obtained from upscaling the $T$ sourcing have highlighted the role that the saturated zone plays in explaining the hydrological behaviour of Q.i. and Q.p.- ultimately highlighting its importance for their survival in extreme dry conditions.

\subsubsection{Species-related spatial patterns of $T_{\text {sta-g }}$ and $T_{\text {sta-u }}$}

A step further in the analysis of the $T_{\text {sta-g }}$ and $T_{\text {sta-u }}$ sourcing leads to analysing the role and/or implications of species-specific traits in the transpiration dynamics across the catchment. Q.i. and Q.p. are both trees of the same genus (Quercus) but they are different in their wood characteristics, phenology and strategy to cope with the summer drought. The combinations of these conditions have different implications for the landscape and for the hydrology of the catchment. Figure 6.3 shows the $T_{\text {sta-g }}$ and $T_{\text {sta-u }}$ sourcing by species (in $\mathrm{mm} \mathrm{day}^{-1}$ ), while Table 6.3 lists the volumetric rates of water uptake (litres day ${ }^{-1}$ ) from the groundwater $(g)$ and unsaturated zone $(u)$ by species and periods $\left(\mathrm{WET}_{g}, \mathrm{WET}_{u}, \mathrm{DRY}_{g}\right.$ and $\mathrm{DRY}_{u}$ ) for the whole catchment.

First, it is clear from these values and patterns that the species location in the catchment determines the spatial patterns of $T_{\text {sta-g }}$ and $T_{\text {sta-u }}$. For example, Q.i. can be found in most parts of the catchment but it is especially concentrated on the northeaster side as is evident from higher $T_{\text {sta-g }}$ and $T_{\text {sta-u }}$ in that region for both seasons. In contrast Q.p. is more widely distributed and dispersed, with higher tree concentration close to riverbanks and river lines.

It is interesting to see how average and aggregated water uptake from the unsaturated zone in the WET period for both species is similar (see $\mathrm{WET}_{u}$ Table 6.3), even though Q.p. has almost double the number of 
Table 6.3: Total volume of water (litres day $^{-1}$ ) uptaken from the groundwater $(g)$ and unsaturated zone $(u)$ of al trees per species for the whole Sardón catchment $(\approx 7,546 \mathrm{ha})$, during the WET and DRY periods in the dry-season. Min. is always zero for all cases. Max. is the maximum value recorded in a grid, Sum. is the aggregation of all cells in the grid, Mean is the average value for the grid, and SD. is the standard deviation.

\begin{tabular}{|c|c|c|c|c|c|c|c|c|}
\hline & \multicolumn{2}{|c|}{$\mathrm{WET}_{g}$} & \multicolumn{2}{|c|}{$\mathrm{WET}_{u}$} & \multicolumn{2}{|c|}{$\mathrm{DRY}_{g}$} & \multicolumn{2}{|c|}{$\operatorname{DRY}_{u}$} \\
\hline & Q.i. & Q.p. & Q.i. & Q.p. & Q.i. & Q.p. & Q.i. & Q.p. \\
\hline & 4,464 & 2,233 & 186 & 69 & 1,999 & 1,450 & 2,650 & 852 \\
\hline Sum. & $1,364,424$ & $2,056,611$ & 56,826 & 63,562 & 611,132 & $1,335,729$ & 810,128 & 784,477 \\
\hline Mean & 180.8 & 272.5 & 7.5 & 8.4 & 80.1 & 177.0 & 107.3 & 104.0 \\
\hline SD. & 386.1 & 253.4 & 16.1 & 7.8 & 172.9 & 164.6 & 229.3 & 96.7 \\
\hline
\end{tabular}

trees in the catchment (estimated from satellite imagery; see Chapter 3). The same was observed in the DRY season $\left(\mathrm{DRY}_{u}\right.$ in Table 6.3), when $T_{\text {sta-u }}$ uptake increases to the same extent for both species $(\approx 90 \%)$.

This is the product of two separate factors operating at the stand level. First, Q.i. has larger tree sizes/canopies and fewer but more concentrated tree patches than Q.p., leading to a more intense uptake rate. Second, taking trees of comparable size, Q.i. at the tree level generally abstracts more unsaturated zone water than Q.p. This means that at the stand level Q.i. abstracts water intensively from the unsaturated zone but has fewer trees, whereas Q.p. is less intense but has more trees, thus balancing out the uptake and resulting in a comparable demand.

This finding can be of high interest for forest and catchment management, as fewer but more concentrated patches of Q.i. can absorb more or as much water from the unsaturated zone as larger patches of $Q . p$. trees.

In terms of groundwater uptake, the situation is reversed. For both DRY and WET periods, Q.p. has the highest rates of average and aggregated water uptake from the groundwater (see WET $g$ and $\mathrm{DRY}_{g}$ in Tab. 6.3). This is the case during both periods but it is especially noticeable in the DRY period when aggregated uptake for Q.p. is almost double that of Q.i. (see Table 6.3).

Similar to the spatial patterns of $T_{\text {sta-u }}$, the $T_{\text {sta-g }}$ patterns are mostly related to the unique attributes and strategies displayed by Q.p. (Figure 6.3). For example, Q.p. is sparser but is distributed closer to the river banks where it is easier to tap groundwater and abstracts groundwater water in higher proportions than Q.i., as demonstrated by isotopic readings (see Chapter 5). These observations fit well with the hydrological strategies discussed in previous chapters using other data sources. In Chapter 3 for instance, night flows for Q.p. where discovered, indicating a non-conservative transpiration rate, and thus greater dependence on groundwater. Despite the higher groundwater uptake of Q.p. across the catchment as compared with Q.i., the latter clusters in the northeaster part of the catchment, and thus in that area $T_{\text {sta-g }}$ will be naturally higher for Q.i. than for Q.p. Further research would be needed to understand 
what has caused this clustered pattern, but it is likely that it is a combination of the physical characteristics of the terrain (e.g. based on spatial distribution $Q . i$. trees seem to cluster further from river banks, where water table is deeper) and interspecific competition (i.e. competition for resources between different species) between Q.i. and Q.p.

The higher consumption of groundwater by Q.p. as compared to Q.i. in the dry season is explained by the fact that, as a deciduous tree, it has only spring and summer to accumulate carbohydrates necessary for the production of acorns, and thus be able to reproduce. Therefore, it consumes as much water as it can to ensure that photosynthesis remains active as long as possible.

In contrast, dry season water uptakes for Q.i. are lower in magnitude as it has a more conservative strategy, but consumption might be maintained throughout the year. Future studies should be focused on discretising water sources throughout the whole year in order to define, whether Q.i. has the highest yearly-total groundwater consumption, or instead Q.p..

In terms of hydrological management for Mediterranean areas, the results can help in deriving efficient management practices. A spatially distributed model can be created in which $T$ consumption can be derived as the interaction of the number of trees and the height of the water table. This will facilitate the running of modelling scenarios to investigate situations where the species can be reduced or increased to evaluate changes in $T$ and how contributions from groundwater and unsaturated zones vary.

An example of a management practice could be that both species are planted or thinned out efficiently counter flooding and/or the depletion of groundwater. Although further proof is needed, modelling initiatives such as those proposed by Francés et al. (2011); Tanvir Hassan et al. (2014) and Baird and Maddock (2005) could be a starting point for the inclusion of management scenarios to demonstrate the hydrological management potential of this type of vegetation.

\subsection{Conclusions}

The process of scaling-up $T_{g}$ and $T_{u}$ for Q.i. and Q.p. in the Sardón catchment draws together ecological, evolutionary and hydrological aspects, thus becoming a unique and truly eco-hydrological study.

It was found that $T_{g}$ and $T_{u}$ sourcing varies throughout the dry-season despite stable $T_{c}$. First, during the WET period, the highest sourced water contribution of $T_{c}$ originates from groundwater and it is mostly driven by the uptakes of Q.p.. Later, during the DRY period, groundwater contributions are reduced by half (although still mainly driven by Q.p.) and the contributions from the unsaturated zones are more noticeable with a slightly higher consumption by Q.i.. This observation corresponds well with previously described water-use strategies displayed by Q.i. and Q.p. to survive very-dry water conditions. Further sampling effort 
covering areas with deeper water level is recommended to increase the accuracy of the estimations made in this study.

Moreover, the results indicate how these species, which may have been competing for the same ecological niche, have adapted in such a way that they are surviving an co-habiting in the same area, and how water deficit has been the main driving force behind their adaptation process. As satellite imagery is becoming more available at higher resolutions, there is a great potential for future studies to replicate what was done in the Sardón catchment, specifically in catchments with several tree species. With this knowledge it can now be possible to understand, and at some point even foresee, what the impact on $T$ and ultimately on the vegetation will be if the water depth table is greatly reduced or if the vegetation is removed.

Studying subsurface hydrological processes at various spatio-temporal scales is a complex and challenging task. Nevertheless, this approach provides a rich amount of information that increases understanding of plant-water interactions, ultimately leading to improved water management practices. 

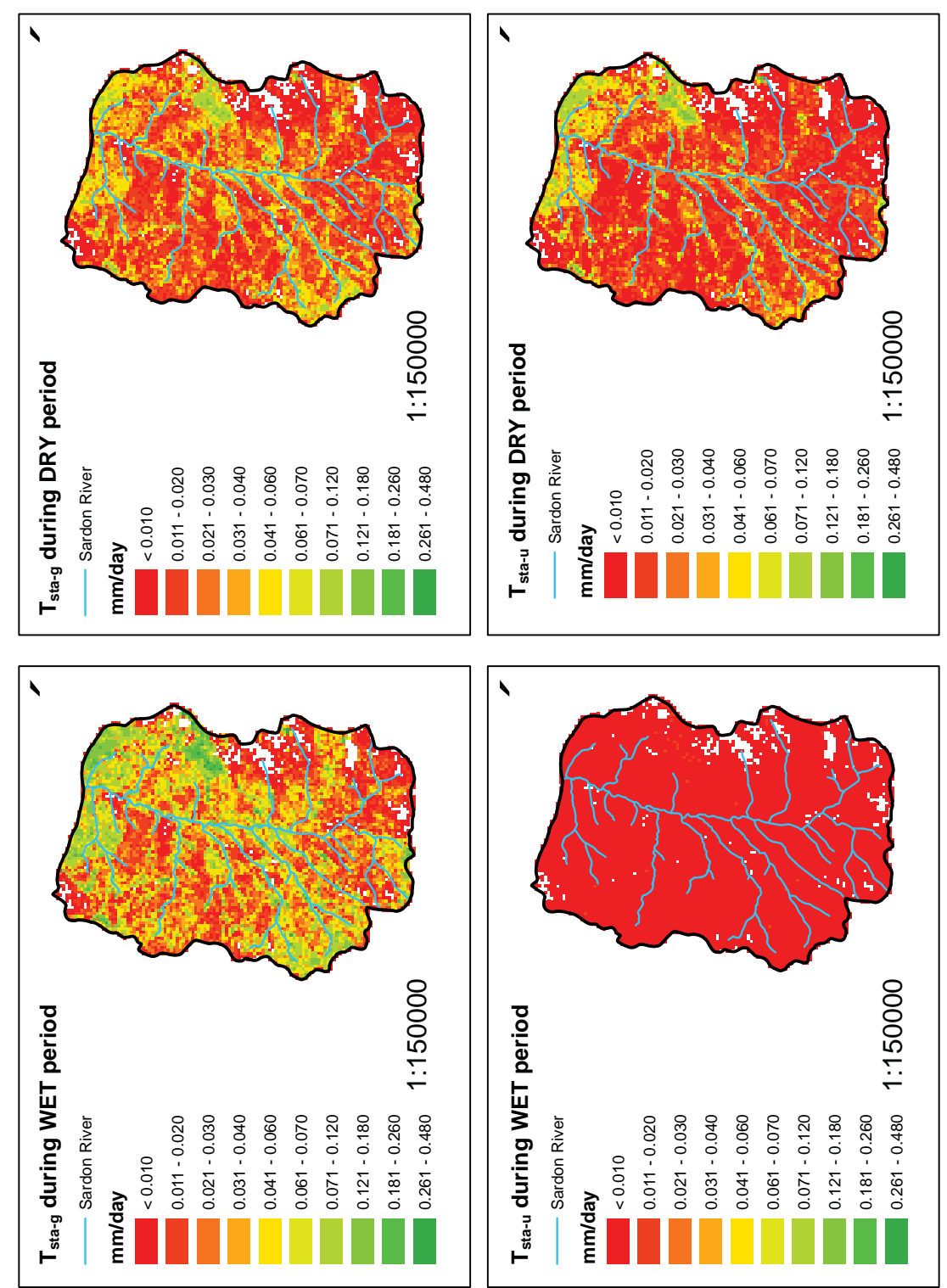

푸

을

ฮิ

동

త্ৰ

릉

采

草

㫯

్ㅡㄹ

画

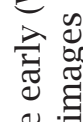

물

罡

苞苞

氠

ช

들 흠

$\stackrel{\Xi}{ \pm}$

$\Xi \approx$

을

롴

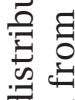

告要

क

串

สิ

站

ij

0

渮

汪 

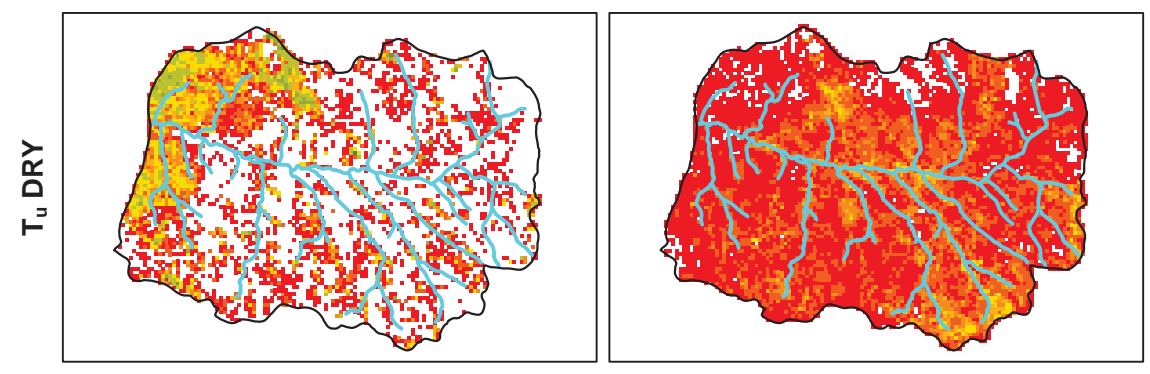

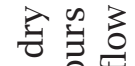

글 苟

范

冚

矛兽

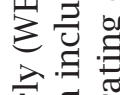

ฮี ซี

월
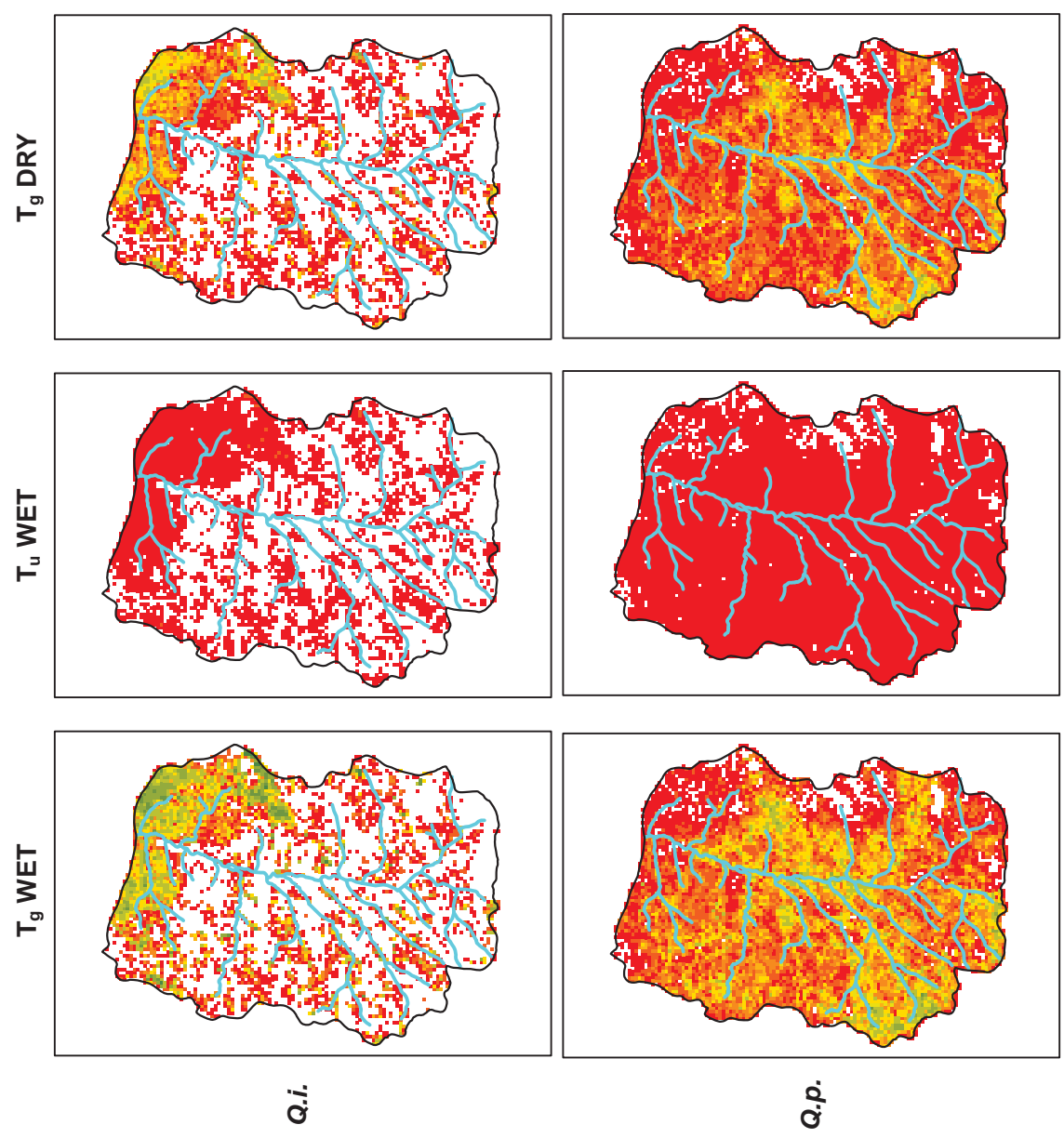

国

害兄

를

घี

氜品

정 3

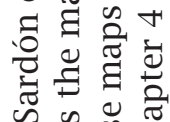

की

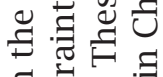

$\exists \because$.

ㄷํㅇ

记

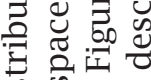

: की

홀

च क के

สิ ีี

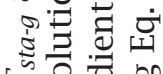

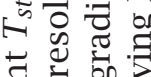

흘

卷

웜

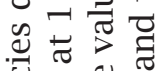

$\ddot{\circ}$

की

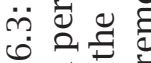

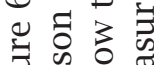

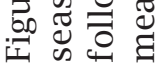




\subsection{Appendix}
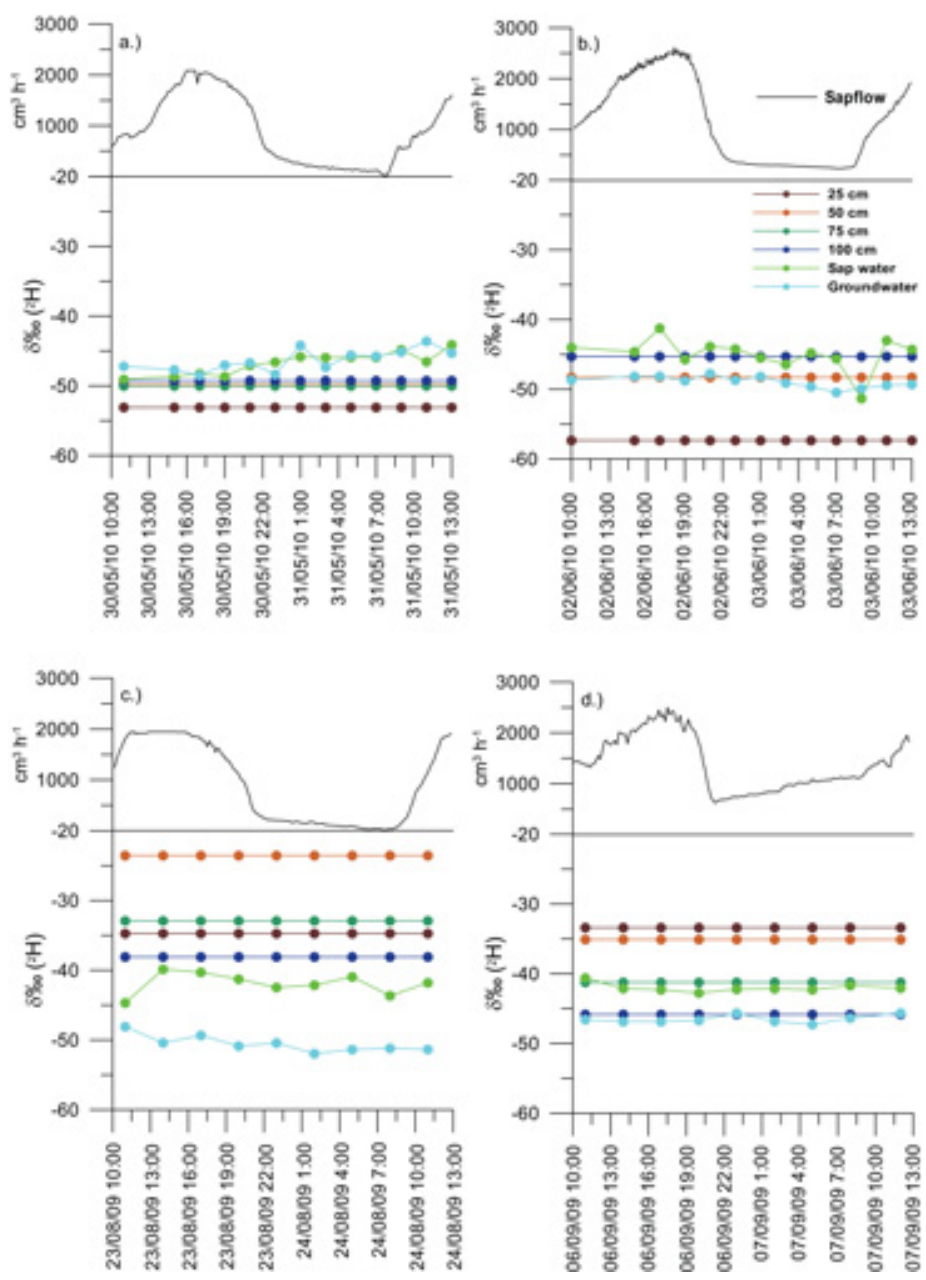

Figure 6.4: Deuterium and sap flow measurements during WET (a. Q.i. and b. Q.p.) and DRY (c. Q.i. and d. Q.p.) periods in trees of the most typical size in Sardón. The reduction in the isotopic concentration of the soil from WET (higher humidity and higher water table) to DRY (lower humidity and lower water table) indicates a shift in soil moisture content and thus of water consumption. Coloured lines represent the different water sources sampled (soil depths, groundwater and sap water), and the black line is sap flow. Time axes follow Spanish summer time, GMT+1, which is approximately 2.5 hours ahead of the geographical ("solar") time. 



\section{Summary}




\subsection{Research setup}

The subject of the present doctoral thesis draws on a growing global concern, namely the increasing scarcity of water resources. Inadequate management of the vegetation could be a significant contributor to this situation. Such a state of affairs calls for a more adequate assessment and quantification of the components of transpiration, the main mechanism through which water is abstracted by vegetation. An appropriate management of the vegetation is of particular importance for environments poor in water - better defined as water limited environments (WLEs) - where water abstraction from groundwater sources is a typical element of tree-water interactions and one that can even influence the evolution of the vegetation in such environments.

The choice of this research topic is further justified by the increasing problem of climate and land cover changes, which reinforces the need for a more thorough understanding of WLEs, especially given the considerable number of people increasingly inhabiting these areas (Seckler and International Water Management, 1998; Brown et al., 2005). Therefore, in order to use water and vegetation efficiently in a climate and land-cover change context, research into the ecohydrological role of vegetation to gain greater understanding of the water dynamics in ecosystems has a critical part to play, not only in the future WLEs but also in the overall impact on the ecosphere.

In order to draw a more comprehensive picture of the links between the various aspects involved in the study of tree water uptake from the subsurface (e.g. groundwater and the unsaturated zone of the subsurface), or more precisely of the ecohydrological processes involved, this study has relied on a holistic methodology designed across a variety of stages. The aim was to provide more information, as well as comprehensive orientations, on both the ecohydrological dynamics of trees and on a series of methodological guidelines for steps to be taken to measure their water-use dynamics in WLEs. This dissertation is thus structured into six chapters mirroring the methodological milestones of this study: the first introduces the research topic; the second consists of a detailed presentation of the laboratory work carried out to appropriately measure water flow (i.e sap flow) inside trees without thermal biases (critical for many sap flow measuring sensors); the third focuses on field sap flow measurements and how it is critical to use more than one technique/sensor to cover the complexity of water movement in trees; the fourth describes the upscaling of sap flow measurements by means of remote sensing, an important step to facilitate the interpretation of these measurements at larger organisational scales; the fifth discusses the use of isotope tracing and plant water models for sourcing tree transpiration and understanding the mechanisms governing water uptake; finally, the sixth presents an attempt of upscaling the sourced transpiration components, providing a novel view of water dynamics at large spatial scales. 


\subsection{Methodological milestones}

Relying on an adequate methodology, and acknowledging the important place and role that vegetation has in WLEs, as well as the impact it is likely to have upon hydrological resources, this thesis aimed at accounting for the dynamics of tree transpiration, as well as the water uptake at a three spatial scales, namely tree, stand and catchment. The area chosen for the presented analysis was the Sardón catchment $\left(\approx 80 \mathrm{~km}^{2}\right)$, located $\approx 30$ $\mathrm{km}$ west of Salamanca, Spain. This choice was justified by the availability of a hydrological monitoring network and the multidisciplinary research carried out in this catchment, the semi-arid climate and related water scarcity, and finally the phreatophytic open woodland type of ecosystem locally called "Dehesas", which is representative of central-western Spain (David et al., 2004; Moreno et al., 2005). The climatic conditions characterising "Dehesas" throughout the dry season can be comparable to various WLE zones throughout the world, and therefore many of the results obtained in this study might be of relevance for other types of dry environments. In particular, this research focused on two phreatophyte tree species in the study area, evergreen Quercus ilex v Desf. Samp. (Q.i.) and deciduous Quercus pyrenaica Willd (Q.p.) oak trees.

Reaching the targets of this study has involved the design of a whole range of technical and methodological aspects concerning the tree water flow measurement (i.e. sap flow). In this way, the study purports to reveal the biasing impact of natural temperature gradients (NTGs) on the effectiveness of the thermal dissipation probes (TDP) method, while checking and validating the cyclic heat dissipation (CHD) method with a view to its correction and validation. Providing more information on the bias posed by NTGs, the study also envisaged testing the CHD method elaborated within the Water Department of the Faculty of Geoinformation Science and Earth Observation of the University of Twente. The obtained results are of great importance for the field of sap flow measurement, because for the first time empirical evidence has shed light on the understanding and correction (by CHD) of the NTG biasing effect, which affects the measurements schemes of many researchers around the globe.

Later the study focused on taking the learning from the laboratory to the field, with the aim of elaborating an efficient and accurate quantification of the sap flow in quasi-arid conditions for Q.i. and Q.p. To achieve this, a methodological approach was designed for the optimisation of numerous TDP sap flux density measurements, in combination with complementary within-stem heat field deformation (HFD) measurements. In this study, several aspects pertaining to inaccuracies while using sap flow measuring techniques were addressed and alternatives proposed. These efforts were equally directed at finding additional information on the dynamics of water uptake by these species at the tree level.

In order to account for transpiration dynamics and the subsequent spatial implications, an adequate set of remote sensing techniques were 
aligned with field sap flow measurements to estimate, as efficiently and as accurately as possible, sap flow and tree transpiration at large scales. To achieve this accurate image of variation across the studied scales, especially since sap flow rates and water requirements may vary, the number of trees was identified and quantified using high-resolution remote sensing for the particular scale, and then a sufficient number of biometric characteristics was defined to allow transpiration measurements at the tree level to be reinterpreted at higher scales. In addition to these steps, it was necessary to describe a modelling approach appropriate for filling in gaps in the data and to compute the uncertainties likely to appear during the measurements.

A further aim of this doctoral study was also to provide a broad explanation of the dynamics regulating the uptake of water from different hydrological sources and the underlying dynamics driving that uptake. In order to achieve this, the study focused on identifying the contributions of the groundwater and the unsaturated zone of the subsurface to the Q.i. and Q.p. transpiration. This was achieved by performing a series of experiments aimed at tracing the stable isotopes, while making use of the Steppe et al. (2006) model as a research basis to explain their contributions. The experiment focused on measurements taking place at the start of the dry season, when the leaves appear on Q.p. Then, these measurements were compared with measurements taken towards August-September, the time when the dry season comes to an end and when soil moisture is shown to have the lowest values.

An additional goal was to determine, by assessing the contribution of the various water sources, the spatial transpiration source dynamics of the identified contributions within the studied species (Q.i. and Q.p.). To this end, based on the application of the developed tree transpiration upscaling techniques, this study has equally tried to reveal the spatial water variation and its contribution by using remote sensing.

In short, the methodological keys to properly comprehending the hydrological differences and water-use strategies of the catchment's tree species consist of steady measurement of tree transpiration, determination of source contributions to that transpiration, and then extrapolation to different spatial scales.

\subsection{Ecohydrological milestones}

The approach of combining techniques from different disciplines to study tree transpiration in Q.i. and Q.p. has unveiled not only a wealth of knowledge that provides an interesting and novel understanding of dry open woodlands, but most of all a starting point for eco hydrological debate.

By applying a sophisticated way of measuring sap flow (i.e. combining CHD with HFD measurements), several insights into the complexity of water movement in trees were unveiled, along with a sound data set of sap flow measurements for Q.i. and Q.p.. The most interesting ones 
related to the different strategies adopted by Q.i. and Q.p. to abstract water and survive very dry conditions. For example, Q.p. maintained a high transpiration rate throughput the season which led to nightly flow of water, presumably to replenish storage-water from the stem. Q.i. on the other hand, had a lower transpiration rate than Q.p. and a much less significant night flow. Radial sap flow differences were also found in Q.i. and Q.p. indicating a more active water transport within the stem, and no difference in uptake rate between different tree sizes, amongst others. All these traits suggest $Q . p$. uptake water more actively than $Q . i$. and thus a higher demand on the hydrology of the catchment.

In the next stages of the research, results from scaling up sap flow with satellite imagery provided a thorough insight into the mechanisms that regulate water use at higher spatial scales but, more importantly, that generate the spatial vegetation patterns observed and assessed in the images. For those observations was found: (1)Q.p. tree patches closely follow main watercourses, and ephemeral rivers whereas $Q . i$. trees tend to cluster further away from them; (2) tree density of Q.p. is the highest in the catchment $(\approx 70 \%$ more trees than $Q . i$.$) and therefore$ Q.p. is the main contributor to dry-season transpiration in the catchment; (3) Q.i. with a smaller density still contributes at least a third of it. The information obtained can be of benefit in the development, validation and calibration of hydrological models and, more broadly, in the design of water management policies.

In the late stages of the study, efforts were made to identify the way in which water source elements of the tree transpiration are used. The findings from tracing naturally abundant isotopes to define water source contributions suggest that water uptake from the groundwater is at its peak at the beginning of the dry season, when the water table is close to its highest yearly peak, whereas abstraction from the unsaturated zones seems to be focused on the late dry-season, for both Q.i. and Q.p., when the water table is deepest. Moreover, Q.p. was defined as the most dependent on groundwater sources, and the modelling framework identified storage as a key dynamic to maintain transpiration under the driest conditions for both Q.i. and Q.p. These results are in agreement with night-flow episodes and stem-variability observations from field measurements.

Finally, upscaling the tree transpiration source components has revealed that groundwater is a key element for the survival of Q.i. and Q.p., but more importantly that their consumption is localised and varies spatially across the Sardón catchment, most of the times following the paths of the ephemeral rivers or the river banks in the valleys. In addition, water uptake values for Q.i. at the end of the dry season suggest that this species might have a higher influence on the humidity conditions of the unsaturated zone, as a small number of these trees have almost the same uptake as all the Q.p. trees in the catchment (one third more trees than Q.i.). 


\subsection{Final conclusions}

This PhD study manages to prove that reliable estimation of transpiration at various spatio-temporal scales can be achieved through adequate use of sap flow measurement and the upscaling of transpiration from remotely sensed imagery. More importantly, however, it shows that detailed measurement of these can provide critical information about the vegetation processes influencing the hydrological systems in very dry conditions.

As regards adequate sap flow measuring techniques, we concluded that the TDP method is susceptible to NTGs, especially at low flows, but that the CHD method successfully corrected the TDP signal and resulted in generally more accurate sap flux density estimates than other cyclic power methods. Exploring optimised field sap flow measurements revealed a novel set of physiological traits and flow dynamics that must be addressed to properly account for water flow in Q.i. and Q.p., and to achieve this, an optimal approach is to combine the strengths of complementary methods such as TDP, CHD and HFD.

The remote sensing upscaling approach proved to be a very efficient way of extrapolating the sap flow measurements, and this exercise allowed us to identify how both species importantly influence the hydrological systems by using very specific strategies. On the one hand, Q.p. takes advantage of its proximity to riverbanks to intensely uptake water, whereas $Q$.i. relies more on its sturdiness and conservative uptake of water sources as it is normally further away from the riverbanks. These strategies both have very specific emerging spatial patterns, easily observed in tree transpiration maps.

The considerable importance of employing isotopic tracing measurements and a modelling framework was clearly shown by the revealed insights. It was determined that in an area facing dry-conditions but with shallow groundwater - as in Sardón- vegetation with a long root system (as in the case of oak trees), will uptake water mostly from the groundwater very early in the season. Although groundwater consumption (e.g. $T_{g}$ ) will decrease towards the end of the dry season, it will still be highly relevant at that time. Such behaviour is more typical of riparian species (i.e. plant habitats and communities along the river margins and banks) than of standard woodlands and, consequently, hydrological modelling should be adjusted to reflect such dynamics.

Finally, exploring the scaling up of water contributions further confirmed the contrasting water uptake strategies of Q.i. and Q.p. to survive the summer drought. Moreover, such strategies were also reflected in the spatio-temporal patterns of stand transpiration components from the groundwater and from the unsaturated zone. Such observations are of great value for future guidelines for the management of water resources in Mediterranean areas by thinning or growing more vegetation where necessary. 
Samenvatting

0 


\subsection{Samenvatting}

Boomtranspiratie is het belangrijkste terugkoppelingsmechanisme voor water van elk levend organisme in de waterkringloop. Bomen transpireren miljoenen liters water per dag en in regio's met waterschaarste is zo'n terugkoppeling van cruciaal belang om de waterhuishouding goed te beheren. Bovendien kan boomtranspiratie ook een belangrijk element zijn in de ondersteuning van watermanagement in gebieden waar hevige overstromingen voorkomen.

Dit proefschrift richt zich op de kwantificering van de boomtranspiratie van twee phreatophyte soorten in een open bosgebied en bestudeert hun strategieën voor watergebruik. Deze phreatophyten zijn de wintergroene Quercus ilex Desf. Samp. (Q.i.) en de loofverliezende Quercus pyrenaica Wild. (Q.p.). Beiden behoren tot het geslacht Quercus (eik) en komen voor in het stroomgebied van de Sardón in de Provincie Salamanca (Spanje). Deze studie aan boomtranspiratie voor deze soorten maakte gebruik van verschillende tijdsgebonden, ruimtelijke schalen, waarvoor het gebruik van satellietbeelden van fundamenteel belang was. Bovendien werden met behulp van isotooptracering de bronnen, van waaruit het getranspireerde water werd opgenomen, geïdentificeerd en opgeschaald naar het niveau van stroomgebieden.

De studie bestond uit een reeks van onderzoeksfasen die oplossingen gaven voor verschillende methodologische uitdagingen. Zodoende konden transpiratie gekwantificeerd worden en tegelijkertijd werden strategieën geïdentificeerd voor watergebruik van Q.i. en Q.p die bruikbaar waren in het verdere onderzoek naar waterbeheer. In een eerste fase werden laboratoriumexperimenten uitgevoerd om te corrigeren voor verloop in thermometrische apparatuur (thermische dissipatie sondes of TDPs) bij de meting van waterstroom in bomen (formeel gedefinieerd als "sapflow") veroorzaakt door natuurlijke thermische gradiënten. In de tweede fase werd een innovatief design voor veldonderzoek toegepast om om te kunnen gaan met de complexiteit van het meten van sapflow in eikenspecies. Zo kon beter inzicht verkregen worden in de verschillen en overeenkomsten tussen $Q . i$. en $Q . p$., en werd een rijke dataset gegenereerd, die bruikbaar was voor verder onderzoek. In de derde fase konden, door het gebruik van satellietbeelden, de veldmetingen worden opgeschaald van boomniveau naar dat van bosbestanden en uiteindelijk naar het niveau van stroomgebieden. De resultaten van deze fase leverden de eerste ruimtelijke weergave van de boomtranspiratie in de hele regio, identificeerde hot spots van transpiratie en gaven meer gedetailleerde informatie over de water-gebruik strategieën van Q.i. en Q.p., in relatie tot de afstand van waterbronnen.

Additioneel veldonderzoek richtte zich op isotoop-tracerings experimenten die geoptimaliseerd waren voor hoog-temporele resolutie. De resultaten van deze experimenten gaven aanwijzingen voor de verhoudingen in de opname van ondergrondse lagen (bijvoorbeeld grondwater en de onverzadigde zone) en leverden daarbij ook een eerste impressie 
over hun tijdsgebonden variabiliteit en hoe dit gerelateerd is aan het grondwaterniveau. Deze resultaten werden verder geanalyseerd met een modelmatige benadering, waaruit werd geconcludeerd dat $Q$.i.- en Q.p.-bomen in deze regio zich niet gedragen als typische eikenbos bomen maar meer als oevervegetatie.

Tenslotte werden methodologische leer- en datasets samengevoegd in een eerste poging (voor zover de auteur bekend) om tijdsgebonden kaarten te construeren van de bijdragen van het grondwater en de verzadigde zone voor Q.i. en Q.p.. Deze kaarten zijn van grote waarde om de transpiratie-dynamiek en -intensiteit van deze soorten te kunnen begrijpen, maar ook door de ruimtelijke weergave die ze geven van de hydrologische configuratie in het Sardón stroomgebied.

De resultaten in dit promotie onderzoek tonen aan dat de betrouwbaarheid van de inschatting van transpiratie op verschillende ruimtelijke en tijdsgebonden schalen bereikt kan worden door adequaat gebruik van sapstroom metingen en door de opschaling van transpiratie van op afstand-gemeten beelden. Nog belangrijker is dat deze gedetailleerde meting informatie oplevert over de vegetatieprocessen die de hydrologische systemen beïnvloeden in zeer droge omstandigheden.

Dergelijke waarnemingen zijn van grote waarde voor toekomstige richtlijnen voor het beheer van de watervoorraden in Mediterrane gebieden, door middel van het kweken of het uitdunnen van vegetatie waar dat nodig is. 



\section{Bibliography}

Abrahams, A. D. and Parsons, A. J. (1994). Geomorphology of deserts environments in Geomorphology of Deserts Environments edited by A.D Abrahams and A.J. Parsons, p. 1-12. Chapman \& Hall, London; New York.

Abramowitz, G. (2005). Towards a benchmark for land surface models. Geophys. Res. Lett, 32(L22702):L22702.

Allen, A. and Chapman, D. (2001). Impacts of afforestation on groundwater resources and quality. Hydrogeology Journal, 9(4):390-400.

Allen, R., Pereira, L., Raes, D., and Smith, M. (1998). Crop evapotranspiration-Guidelines for computing crop water requirementsFAO Irrigation and drainage paper 56. FAO, Rome, 300:6541.

Amenu, G. G. and Kumar, P. (2007). A model for hydraulic redistribution incorporating coupled soil-root moisture transport. Hydrology and Earth System Sciences Discussions, 4(5):3719-3769.

Baatz, M., Benz, U., Dehghani, S., Heynen, M., Holtje, A., Hofmann, P., Lingenfelder, I., Mimler, M., Sohlbach, M., and Weber, M. (2001). ecognition object oriented image analysis. User Guide, Definiens Imaging, München, Germany.

Baird, K. J. and Maddock, T. (2005). Simulating riparian evapotranspiration: a new methodology and application for groundwater models. Journal of Hydrology, 312(1-4):176-190.

Balugani, E., Reyes-Acosta, J. L., van der Tol, C., Francés, A. P., and Lubczynski, M. W. (2011). Partitioning and sourcing of dry season evapotranspiration fluxes at the footprint of the eddy covariance tower in Sardón semi-arid location in Spain. ZNS conference proceedings.

Batelaan, O. and De Smedt, F. (2007). GIS-based recharge estimation by coupling surface-subsurface water balances. Journal of Hydrology, 337(3-4):337-355.

Batelaan, O., De Smedt, F., and Triest, L. (2003). Regional groundwater discharge: phreatophyte mapping, groundwater modelling and impact analysis of land-use change. Journal of Hydrology, 275(1-2):86-108.

Bernier, P., Bréda, N., Granier, A., Raulier, F., and Mathieu, F. (2002). Validation of a canopy gas exchange model and derivation of a soil 
water modifier for transpiration for sugar maple (acer saccharum marsh.) using sap flow density measurements. Forest Ecology and Management, 163(1-3):185-196.

Blom, C. and Voesenek, L. (1996). Flooding: the survival strategies of plants. Trends in Ecology \& Evolution, 11(7):290-295.

Boegh, E., Soegaard, H., Hanan, N., Kabat, P., and Lesch, L. (1999). Remote Sensing of Environment, 69(3):224-240.

Braun, P. and Schmid, J. (1999). Sap flow measurements in grapevines (vitis vinifera 1.) 2. granier measurements. Plant and Soil, 215(1):47-55.

Bredemeier, M., Cohen, S., Godbold, D. L., Lode, E., Pichler, V., and Schleppi, P. (2011). Forest Management and the Water Cycle. Springer.

Brigati, G., Lucarini, M., Mugnaini, V., and Pedulli, G. F. (2002). Determination of the substituent effect on the $\mathrm{OH}$ bond dissociation enthalpies of phenolic antioxidants by the EPR radical equilibration technique. The Journal of Organic Chemistry, 67(14):4828-4832.

Brooks, J. R., Meinzer, F. C., Coulombe, R., and Gregg, J. (2002). Hydraulic redistribution of soil water during summer drought in two contrasting Pacific Northwest coniferous forests. Tree Physiology, 22(15-16):110717.

Brown, A. E., Zhang, L., McMahon, T. A., Western, A. W., and Vertessy, R. A. (2005). A review of paired catchment studies for determining changes in water yield resulting from alterations in vegetation. Journal of Hydrology, 310:28 - 61 .

Brunel, J.-P., Walker, G., Dighton, J., and Monteny, B. (1997). Use of stable isotopes of water to determine the origin of water used by the vegetation and to partition evapotranspiration. a case study from hapex-sahel. Journal of hydrology, 188:466-481.

Brunel, J.-P., Walker, G. R., and Kennett-Smith, A. K. (1995). Field validation of isotopic procedures for determining sources of water used by plants in a semi-arid environment. Journal of Hydrology, 167(1-4):351368.

Burgess, S. S. O., Adams, M. A., and Bleby, T. M. (2000). Measurement of sap flow in roots of woody plants: a commentary. Tree Physiology, 20(13):909-913.

Burgess, S. S. O., Adams, M. A., Turner, N. C., Beverly, C. R., Ong, C. K., Khan, A. A. H., and Bleby, T. M. (2001). An improved heat pulse method to measure low and reverse rates of sap flow in woody plants. Tree Physiology, 21(9):589-598.

Burgess, S. S. O. and Bleby, T. M. (2006). Redistribution of soil water by lateral roots mediated by stem tissues. Journal of Experimental Botany, 57:3283-3291. doi:10.1093/jxb/erl085.

Burgess, S. S. O. and Dawson, T. E. (2004). The contribution of fog to the water relations of Sequoia sempervirens (D. Don): foliar uptake and prevention of dehydration. Plant, Cell \& Environment, 27(8):1023-1034. 
Bush, S. E., Hultine, K. R., Sperry, J. S., and Ehleringer, J. R. (2010). Calibration of thermal dissipation sap flow probes for ring- and diffuseporous trees. Tree Physiology, 30(12):1545-1554.

Calder, I. R. (1978). Transpiration observations from a spruce forest and comparisons with predictions from an evaporation model. Journal of Hydrology, 38(1-2):33-47.

Canadell, J., Jackson, R., Ehleringer, J., Mooney, H., Sala, O., and Schulze, E.-D. (1996). Maximum rooting depth of vegetation types at the global scale. Oecologia, 108(4):583-595.

Carreiras, J. M. B., Pereira, J. M. C., and Pereira, J. S. (2006). Estimation of tree canopy cover in evergreen oak woodlands using remote sensing. Forest Ecology and Management, 223(1-3):45-53.

Caspari, H. W., Green, S. R., and Edwards, W. R. N. (1993). Transpiration of well-watered and water-stressed asian pear trees as determined by lysimetry, heat-pulse, and estimated by a penman-monteith model. Agricultural and Forest Meteorology, 67(1-2):13-27.

Cavanaugh, M. L., Kurc, S. A., and Scott, R. L. (2011). Evapotranspiration partitioning in semiarid shrubland ecosystems: a two-site evaluation of soil moisture control on transpiration. Ecohydrology, 4(5):671-681.

Caylor, K. K. and Dragoni, D. (2009). Decoupling structural and environmental determinants of sap velocity: Part i. methodological development. Agricultural and Forest Meteorology, 149(3-4):559-569.

Čermák, J., Deml, M., and Penka, M. (1973). A new method of sap flow rate determination in trees. Biologia Plantarum, 15(3):171-178.

Čermák, J., Hruska, J., Martinková, M., and Prax, A. (2000). Urban tree root systems and their survival near houses analyzed using ground penetrating radar and sap flow techniques. Plant and Soil, 219(1):103116.

Čermák, J., Kučera, J., Bauerle, W. L., Phillips, N., and Hinckley, T. M. (2007). Tree water storage and its diurnal dynamics related to sap flow and changes in stem volume in old-growth douglas-fir trees. Tree Physiol, 27(2):181-198.

Čermák, J. and Kučera, J. (1981). The compensation of natural temperature gradient at the measuring point during the sap flow rate determination in trees. Biologia Plantarum, 23(6):469-471.

Čermák, J. and Kučera, J. (1990). Scaling up transpiration data between trees, stands and watersheds. Silva Carelica, 15:101-120.

Čermák, J., Kučera, J., and Nadezhdina, N. (2004). Sap flow measurements with some thermodynamic methods, flow integration within trees and scaling up from sample trees to entire forest stands. Trees - Structure and Function, 18(5):529-546.

Čermák, J. and Nadezhdina, N. (1998). Sapwood as the scaling parameter defining according to xylem water content or radial pattern of sap flow. 55(5):509-521. 
Čermák, J., Nadezhdina, N., Meiresonne, L., and Ceulemans, R. (2008). Scots pine root distribution derived from radial sap flow patterns in stems of large leaning trees. Plant and Soil, 305(1-2):61-75.

Čermák, J., Riguzzi, F., and Ceulemans, R. (1998). Scaling up from the individual tree to the stand level in Scots pine. I. Needle distribution, overall crown and root geometry. 55(1-2):63-88.

Čermák, J., Ulehla, J., Kučera, J., and Penka, M. (1982). Sap flow rate and transpiration dynamics in the full-grown oak (quercus robur l.) in floodplain forest exposed to seasonal floods as related to potential evapotranspiration and tree dimensions. Biol. Plant, 24:446-460.

Chavarro-Rincón, D. (2009). Tree Transpiration Mapping from Upscaled Sapflow in the Botswana Kalahari. PhD thesis.

Chu, C. R., Hsieh, C.-I., Wu, S.-Y., and Phillips, N. G. (2009). Transient response of sap flow to wind speed. Journal of Experimental Botany, 60(1):249-255.

Cienciala, E., Kucera, J., and Malmer, A. (2000). Tree sap flow and stand transpiration of two acacia mangium plantations in sabah, borneo. Journal of Hydrology, 236(1-2):109-120.

Clearwater, M. J., Meinzer, F. C., Andrade, J. L., Goldstein, G., and Holbrook, N. M. (1999). Potential errors in measurement of nonuniform sap flow using heat dissipation probes. Tree Physiology, 19(10):681687.

Cochran, W. (1977). Sampling techniques 3 Ed. John Wiley \& Sons, New York.

Cohen, J. (1960). A coefficient of agreement for nominal scales. Educational and Psychological Measurement, 20(1):37-46.

Congalton, R. (1996). Accuracy assessment: a critical component of land cover mapping. In Scott, J. M., Tear, T. H., and Davis, F. W., editors, Gap analysis: a landscape approach to biodiversity planning., pages 119-131. American Society for Photogrammetry and Remote Sensing.

Congalton, R. G. (1991). A review of assessing the accuracy of classifications of remotely sensed data. Remote Sensing of Environment, 37(1):35-46.

Congalton, R. G. and Green, K. (1999). Assessing the accuracy of remotely sensed data: Principles and practices. PBD: 1999. Lewis Publishers, Boca Raton, FL (United States), United States.

Cook, P. and O'Grady, A. (2006). Determining soil and ground water use of vegetation from heat pulse, water potential and stable isotope data. Oecologia, 148(1):97-107. 10.1007/s00442-005-0353-4.

Coplen, T. B., Wildman, J. D., and Chen, J. (1991). Improvements in the gaseous hydrogen-water equilibration technique for hydrogen isotoperatio analysis. Analytical Chemistry, 63(9):910-912.

Corcuera, L., Camarero, J., and Gil-Pelegrín, E. (2004). Effects of a severe drought on quercus ilex radial growth and xylem anatomy. Trees Structure and Function, 18(1):83-92. 
Cristóbal, J., Poyatos, R., Ninyerola, M., Llorens, P., and Pons, X. (2011). Combining remote sensing and GIS climate modelling to estimate daily forest evapotranspiration in a Mediterranean mountain area. Hydrology and Earth System Sciences, 15(5):1563.

Cummins, B. (2007). Biological Science. (3 ed) edition.

Dale, V. H. (2003). Ecological Modeling for Resource Management. Springer-Verlag, New York.

David, T. S., Ferreira, M. I., Cohen, S., Pereira, J. S., and David, J. S. (2004). Constraints on transpiration from an evergreen oak tree in southern Portugal. Agricultural and Forest Meteorology, 122(3-4):193-205.

David, T. S., Henriques, M. O., Kurz-Besson, C., Nunes, J., Valente, F., Vaz, M., Pereira, J. S., Siegwolf, R., Chaves, M. M., Gazarini, L. C., and David, J. S. (2007). Water-use strategies in two co-occurring Mediterranean evergreen oaks: surviving the summer drought. Tree Physiology, 27:793-803.

David, T. S., Pinto, C. A., Nadezhdina, N., Kurz-Besson, C., Henriques, M. O., Quilhó, T., Cermak, J., Chaves, M. M., Pereira, J. S., and David, J. S. (2013). Root functioning, tree water use and hydraulic redistribution in quercus suber trees: A modeling approach based on root sap flow. Forest Ecology and Management, 307(0):136 - 146.

Dawson, T. E., Mambelli, S., Plamboeck, A. H., Templer, P. H., and Tu, K. P. (2002). Stable isotopes in plant ecology. Annual Review of Ecology and Systematics, 33(1):507-559.

De Pauw, D. J. W., Steppe, K., and De Baets, B. (2008). Identifiability analysis and improvement of a tree water flow and storage model. Mathematical Biosciences, 211(2):314-332.

Delzon, S., Sartore, M., Granier, A., and Loustau, D. (2004). Radial profiles of sap flow with increasing tree size in maritime pine. Tree Physiology, 24(11):1285-1293.

Devitt, D., Berkowitz, M., Schulte, P., and Morris, R. (1993). Estimating transpiration for three woody ornamental tree species using stem-flow gauges and lysimetry. HortScience, 28:320-320.

Do, F. and Rocheteau, A. (2002a). Influence of natural temperature gradients on measurements of xylem sap flow with thermal dissipation probes. 1. Field observations and possible remedies. Tree Physiology, 22(9):641-648.

Do, F. and Rocheteau, A. (2002b). Influence of natural temperature gradients on measurements of xylem sap flow with thermal dissipation probes. 2. Advantages and calibration of a noncontinuous heating system. Tree Physiology, 22(9):649-654.

Dorren, L. K. A., Maier, B., and Seijmonsbergen, A. C. (2003). Improved landsat-based forest mapping in steep mountainous terrain using object-based classification. Forest Ecology and Management, 183(13):31-46. 
Dragoni, D., Caylor, K. K., and Schmid, H. P. (2009). Decoupling structural and environmental determinants of sap velocity: Part ii. observational application. Agricultural and Forest Meteorology, 149(3-4):570-581.

Edwards, W. R. N., Becker, P., and Čermák, J. (1997). A unified nomenclature for sap flow measurements. Tree Physiology, 17(1):65-67.

Edwards, W. R. N., Jarvis, P. G., Landsberg, J. J., and Talbot, H. (1986). A dynamic model for studying flow of water in single trees. 1:309-324.

Ehleringer, J. R., Roden, J., and Dawson, T. E. (2000). Assessing Ecosystem - level Water Relations through Stable Isotope Ratio Analyses. Academic, San Diego, CA.

Ehlers, M., Gahler, M., and Janowsky, R. (2003). Automated analysis of ultra high resolution remote sensing data for biotope type mapping: new possibilities and challenges. ISPRS Journal of Photogrammetry and Remote Sensing, 57(5-6):315-326.

Ellsworth, P. and Williams, D. (2007). Hydrogen isotope fractionation during water uptake by woody xerophytes. Plant and Soil, 291(1):93107.

Ewers, B., Mackay, D., and Samanta, S. (2007). Interannual consistency in canopy stomatal conductance control of leaf water potential across seven tree species. Tree Physiology, 27(1):11.

Finnigan, J. (2004). The footprint concept in complex terrain. Agricultural and Forest Meteorology, 127(3):117 - 129. Flux and Concentration Footprints.

Ford, C. R., Hubbard, R. M., Kloeppel, B. D., and Vose, J. M. (2007). A comparison of sap flux-based evapotranspiration estimates with catchment-scale water balance. Agricultural and Forest Meteorology, 145(3-4):176-185.

Ford, C. R., McGuire, M. A., Mitchell, R. J., and Teskey, R. O. (2004) Assessing variation in the radial profile of sap flux density in pinus species and its effect on daily water use. 24:241-249.

Francés, A. P., Reyes-Acosta, J. L., Balugani, E., van der Tol, C., and Lubczynski, M. W. (2011). Towards an improved assessment of the water balance at the catchment scale: a coupled model approach. ZNS conference proceedings, 10:321-326.

Freeze, R. A. and Cherry, J. A. (1979). Groundwater: Englewood Cliffs, New Jersey. EEUU. Prentice Hall.

Gea-Izquierdo, G., Martín-Benito, D., Cherubini, P., and Cañellas, I. (2009). Climate-growth variability in quercus ilex l. west iberian open woodlands of different stand density. Annals of Forest Science, 66(8):802802.

Génard, M., Fishman, S., Vercambre, G., Huguet, J., Bussi, C., Besset, J., and Habib, R. (2001). A biophysical analysis of stem and root diameter variations in woody plants. Plant Physiology, 126(1):188-202. 
Ghimire, C., Bruijnzeel, L., Lubczynski, M., and Bonell, M. (2012). Rainfall interception by natural and planted forests in the Middle Mountains of Central Nepal. Journal of Hydrology (in press).

Giles M, F. (2002). Status of land cover classification accuracy assessment. Remote Sensing of Environment, 80(1):185-201.

Göckede, M., Rebmann, C., and Foken, T. (2004). A combination of quality assessment tools for eddy covariance measurements with footprint modelling for the characterisation of complex sites. Agricultural and Forest Meteorology, 127(3):175 - 188. Flux and Concentration Footprints.

Goulden, M. and Field, C. (1994). Three methods for monitoring the gas exchange of individual tree canopies: ventilated-chamber, sap-flow and penman-monteith measurements on evergreen oaks. Functional Ecology, 8(1):125-135.

Granier, A. (1985). Une nouvelle mèthode pour la mesure du flux de séve brute dans le tronc des arbres. Annuales des Sciences Forestières, 42(2):193-200.

Granier, A. (1987). Evaluation of transpiration in a Douglas-fir stand by means of sap flow measurements. Tree Physiology, 3(4):309-320.

Granier, A. (1996). Comparisons of xylem sap flow and water vapour flux at the stand level and derivation of canopy conductance for Scots pine. Theoretical and Applied Climatology, 53(1):115-122.

Granier, A., Anfodillo, T., Sabatti, M., Cochard, H., Dreyer, E., Tomasi, M., Valentini, R., and Breda, N. (1994). Axial and radial water flow in the trunks of oak trees: a quantitative and qualitative analysis. Tree Physiol, 14(12):1383-1396.

Grime, V. L. and Sinclair, F. L. (1999). Sources of error in stem heat balance sap flow measurements. Agricultural and Forest Meteorology, 94(2):103-121.

Guswa, A. J., Celia, M. A., and Rodriguez-Iturbe, I. (2004). Effect of vertical resolution on predictions of transpiration in water-limited ecosystems. Advances in Water Resources, 27(5):467-480.

Hanasaki, N., Fujimori, S., Yamamoto, T., Yoshikawa, S., Masaki, Y., Hijioka, Y., Kainuma, M., Kanamori, Y., Masui, T., Takahashi, K., et al. (2013). A global water scarcity assessment under shared socioeconomic pathways-part 2: Water availability and scarcity. Hydrology and Earth System Sciences, 17:2393-2413.

Harbaugh, A. W., Banta, E. R., Hill, M. C., and McDonald, M. G. (2000). MODFLOW-2000, the US Geological Survey Modular Ground-water Model: User Guide to Modularization Concepts and the Ground-water Flow Process. US Geological Survey Denver, CO, Reston, VA.

Hatton, T. J. and Wu, H.-I. (1995). Scaling theory to extrapolate individual tree water use to stand water use. Hydrological Processes, 9(5-6):527540. 10.1002/hyp.3360090505. 
Hay, A. (1979). Sampling designs to test land-use map accuracy. Photogrammetric Engineering and remote sensing, 45.

Hernández-Santana, V., David, T. S., and Martínez-Fernández, J. (2008a). Environmental and plant-based controls of water use in a Mediterranean oak stand. Forest Ecology and Management, 255(11):3707-3715.

Hernández-Santana, V., Martínez-Fernández, J., and Moran, C. (2008b). Estimation of tree water stress from stem and soil water monitoring with time-domain reflectometry in two small forested basins in spain. Hydrological Processes, 22(14):2493-2501.

Hernández-Santana, V., Martínez-Fernández, J., Morán, C., and Cano, A. (2008c). Response of Quercus pyrenaica (Melojo oak) to soil water deficit: a case study in Spain. European Journal of Forest Research, 127(5):369-378.

Hernández-Santana, V., Martínez-Vilalta, J., Martínez-Fernández, J., and Williams, M. (2009). Evaluating the effect of drier and warmer conditions on water use by quercus pyrenaica. Forest Ecology and Management, 258(7):1719-1730.

Huguet, J. G. (1985). Appréciation de l'état hydrique d'une plant á partir des variations micrométriques de la dimension des fruits ou des tiges au cours de la journée. Agronomie, 5:753-741.

Hultine, K. R., Scott, R. L., Cable, W. L., Goodrich, D. C., and Williams, D. G. (2004). Hydraulic redistribution by a dominant, warm-desert phreatophyte: seasonal patterns and response to precipitation pulses. Functional Ecology, 18(4):530-538.

Isarangkool Na Ayutthaya, S., Do, F., Pannengpetch, K., Junjittakarn, J., Maeght, J.-L., Rocheteau, A., and Cochard, H. (2010). Transient thermal dissipation method of xylem sap flow measurement: multi-species calibration and field evaluation. Tree Physiology, 30(1):139-148.

Janssen, L., Huurneman, G., Bakker, W., Reeves, C., Gorte, B., Pohl, C., Weir, M., Horn, J., Prakash, A., and Woldai, T. (2001). Principles of remote sensing : an introductory textbook, volume 2 of ITC Educational Textbook Series. ITC, Enschede, second edition edition.

Jones, H. (1992). Plants and Microclimate: A Quantitative Approach to Environmental Plant Physiology. Cambridge Univ Pr.

Kimani, J., Hussin, Y., Lubczynski, M. W., Chavarro, D., and Obakeng, O. (2007). Mapping savannah trees in kalahari using high resolution remotely sensed images and object-oriented classification. International Journal of Geoinformatics, 3(2).

Köstner, B., Granier, A., and Cermak, J. (1998). Sapflow measurements in forest stands: methods and uncertainties. Annales des sciences forestières, 55(1-2):13-27.

Kučera, J., Čermák, J., and Penka, M. (1977). Improved thermal method of continual recording the transpiration flow rate dynamics. Biologia plantarum, 19(6):413-420. 
Kumagai, T., Nagasawa, H., Mabuchi, T., Ohsaki, S., Kubota, K., Kogi, K., Utsumi, Y., Koga, S., and Otsuki, K. (2005). Sources of error in estimating stand transpiration using allometric relationships between stem diameter and sapwood area for cryptomeria japonica and chamaecyparis obtusa. Forest Ecology and Management, 206(1-3):191-195.

Laloy, E., Fasbender, D., and Bielders, C. (2010). Parameter optimization and uncertainty analysis for plot-scale continuous modeling of runoff using a formal bayesian approach. Journal of Hydrology, 380(1-2):8293.

Lambers, H., Chapin, F., Pons, T., and Pospisilova, J. (1998). Plant Physiological Ecology, volume 42 of Biologia Plantarum. Springer-Verlag.

Lambs, L. and Berthelot, M. (2002). Monitoring of water from the underground to the tree: first results with a new sap extractor on a riparian woodland. Plant and Soil, 241(2):197-207. 10.1023/A:1016131102232.

Lhomme, J. P., Rocheteau, A., Ourcival, J. M., and Rambal, S. (2001). Nonsteady-state modelling of water transfer in a Mediterranean evergreen canopy. Agricultural and Forest Meteorology, 108(1):67-83.

Liu, X., Kang, S., and Li, F. (2009). Simulation of artificial neural network model for trunk sap flow of pyrus pyrifolia and its comparison with multiple-linear regression. Agricultural Water Management, 96(6):939945.

Lu, P., Urban, L., and Zhao, P. (2004). Granier's thermal dissipation probe (tdp) method for measuring sap flow in trees: Theory and practice. Acta Botanica Sinica, 46(6):631-646.

Lu, P., Woo, K.-C., and Liu, Z.-T. (2002). Estimation of whole-plant transpiration of bananas using sap flow measurements. Journal of Experimental Botany, 53(375):1771-1779.

Lubczynski, M. W. (2000). Groundwater Evapotranspiration, Underestimated Component of the Groundwater Balance in a Semi - arid Environment, Serowe case, Botswana. Rotterdam.

Lubczynski, M. W. (2009). The hydrogeological role of trees in waterlimited environments. Hydrogeology Journal, 17(1):247-259.

Lubczynski, M. W., Chavarro Rincon, D., and Roy, J. (2012). Novel, cyclic heat dissipation (CHD) method for the correction of natural temperature gradients in sap flow measurements. Part 1. Theory and application. Tree physiology, 32(7):894-912.

Lubczynski, M. W. and Gurwin, J. (2005). Integration of various data sources for transient groundwater modeling with spatio-temporally variable fluxes-Sardon study case, Spain. Journal of Hydrology, 306(14):71-96.

Lundblad, M., Lagergren, F., and Lindroth, A. (2001). Evaluation of heat balance and heat dissipation methods for sapflow measurements in pine and spruce. Ann. For. Sci, 58(6). 
Lundblad, M. and Lindroth, A. (2002). Stand transpiration and sapflow density in relation to weather, soil moisture and stand characteristics. Basic and Applied Ecology, 3(3):229-243.

Manes, F., Vitale, M., Donato, E., Giannini, M., and Puppi, G. (2006). Different ability of three mediterranean oak species to tolerate progressive water stress. Photosynthetica, 44(3):387-393.

Marshall, D. (1958). Measurement of sap flow in conifers by heat transport. Plant Physiology, 33(6):385-396.

Martin, J., Leonard, W., and Stamp, D. (1976). Principles of Field Crop Production. Macmillan Publishing Co., Inc, New York, (3rd edition) edition.

Martínez-Vilalta, J., nol, J. P., and Beven, K. (2002). A hydraulic model to predict drought-induced mortality in woody plants: an application to climate change in the mediterranean. Ecological Modelling, 155:127 147.

McCarthy, E. L. (1934). Mariotte's bottle. Science, 80(2065):100.

McCuen, R., Knight, Z., and Cutter, A. (2006). Evaluation of the nashsutcliffe efficiency index. Journal of Hydrologic Engineering, 11:597.

Mediavilla, S. and Escudero, A. (2003). Stomatal responses to drought at a Mediterranean site: a comparative study of co-occurring woody species differing in leaf longevity. Tree Physiology, 23(14):987-996.

Miller, G., Chen, X., Rubin, Y., Ma, S., and Baldocchi, D. (2010). Groundwater uptake by woody vegetation in a semiarid oak savanna. Water Resources Research, 46(10):W10503.

Moore, D. J. P., Hu, J., Sacks, W. J., Schimel, D. S., and Monson, R. K. (2008). Estimating transpiration and the sensitivity of carbon uptake to water availability in a subalpine forest using a simple ecosystem process model informed by measured net $\mathrm{CO} 2$ and $\mathrm{H} 2 \mathrm{O}$ fluxes. Agricultural and Forest Meteorology, 148(10):1467-1477.

Moreno, G., Gallardo, J. F., and Vicente, M. Á. (2011). How mediterranean deciduous trees cope with long summer drought? the case of quercus pyrenaica forests in western spain. In Bredemeier, M., Cohen, S., Godbold, D. L., Lode, E., Pichler, V., Schleppi, P., Heldmaier, G., Jackson, R. B., Lange, O. L., Mooney, H. A., Schulze, E. D., and Sommer, U., editors, Forest Management and the Water Cycle, volume 212, pages 187-201. Springer Netherlands.

Moreno, G., Obrador, J. J., Cubera, E., and Dupraz, C. (2005). Fine Root Distribution in Dehesas of Central-Western Spain. Plant and Soil, 277(1):153-162.

Murray, R. S., Nagler, P. L., Morino, K., and Glenn, E. P. (2009). An Empirical Algorithm for Estimating Agricultural and Riparian Evapotranspiration Using MODIS Enhanced Vegetation Index and Ground Measurements of ET. II. Application to the Lower Colorado River, U.S. Remote Sensing, 1(4):1125-1138. 
Nadelhoffer, K. J. and Fry, B. (1994). Nitrogen Isotope Studies in Forest Ecosystems. Blackwell Sci, Oxford.

Nadezhdina, N. (1999). Sap flow index as an indicator of plant water status. Tree Physiology, 19(13):885-891.

Nadezhdina, N., Čermák, J., and Ceulemans, R. (2002). Radial patterns of sap flow in woody stems of dominant and understory species: scaling errors associated with positioning of sensors. Tree Physiology, 22:907918.

Nadezhdina, N., Cermak, J., Gasparek, J., Nadezhdin, V., and Prax, A. (2006a). Vertical and horizontal water redistribution in norway spruce (picea abies) roots in the moravian upland. Tree Physiol, 26(10):12771288.

Nadezhdina, N., Čermák, J., Neruda, J., Prax, A., Ulrich, R., Nadezhdin, V., Gašpárek, J., and Pokornỳ, E. (2006b). Roots under the load of heavy machinery in spruce trees. European Journal of Forest Research, 125(2):111-128.

Nadezhdina, N., David, T. S., David, J. S., Ferreira, M. I., Dohnal, M., Tesař, M., Gartner, K., Leitgeb, E., Nadezhdin, V., Cermak, J., et al. (2010). Trees never rest: the multiple facets of hydraulic redistribution. Ecohydrology, 3(4):431-444.

Nadezhdina, N., Ferreira, M., Silva, R., and Pacheco, C. (2008). Seasonal variation of water uptake of a quercus suber tree in central portugal. Plant and Soil, 305(1):105-119.

Nadezhdina, N., Nadezhdin, V., Ferreira, M., and Pitacco, A. (2007). Variability with xylem depth in sap flow in trunks and branches of mature olive trees. Tree Physiol, 27:105-113.

Nadezhdina, N., Steppe, K., De Pauw, D. J. W., Bequet, R., Cermak, J., and Ceulemans, R. (2009). Stem-mediated hydraulic redistribution in large roots on opposing sides of a douglas-fir tree following localized irrigation. New Phytologist, 184(4):932-943.

Nadezhdina, N., Tributsch, H., and Cermák, J. (2004). Infra-red images of heat field around a linear heater and sap flow in stems of lime trees under natural and experimental conditions. Ann. For. Sci., 61(3):203213.

Nadezhdina, N., Vandegehuchte, M., and Steppe, K. (2012). Sap flux density measurements based on the heat field deformation method. Trees structure and function, 26(5):1439-1448.

Nadezhdina, N. J., Čermák, and Nadezhdin., V. (1998). The heat field deformation method for sap flow measurement. In Proc 4th Int Workshop, Zidlochovice, C. R. I. P. B., editor, 4th International Workshop on Measuring Sap flow in Intact Plants. Eds. J. Cérmak and N. Nadezhdina., pages $72-92$.

Nagler, P., Jetton, A., Fleming, J., Didan, K., Glenn, E., Erker, J., Morino, K., Milliken, J., and Gloss, S. (2007). Evapotranspiration in a cottonwood 
(populus fremontii) restoration plantation estimated by sap flow and remote sensing methods. Agricultural and Forest Meteorology, 144:95110.

Newman, B. D., Wilcox, B. P., Archer, S., Breshears, D. D., Dahm, C. N., Duffy, C. J., McDowell, N. G., Phillips, F. M., Scanlon, B. R., and Vivoni, E. R. (2006). Ecohydrology of water-limited environments: a scientific vision. Water Resources Research, 42(6).

Nobel, P. (1999). Physicochemical and Environmental Plant Physiology. Number 8. Academic press.

Nourtier, M., Chanzy, A., Granier, A., and Huc, R. (2011). Sap flow measurements by thermal dissipation method using cyclic heating: a processing method accounting for the non-stationary regime. Annals of Forest Science, 68(7):1255-1264.

Noy-Meir, I. (1973). Desert ecosystems: environment and producers. Annual Review of Ecology and Systematics, 4:25-51. 0066-4162 Article type: Full Length Article / Full publication date: 1973 (1973). / Copyright 1973 Annual Reviews.

Obakeng, O. T., de Vries, J. J. p., and Lubczynski, M. W. p. (2007). Soil Moisture Dynamics and Evapotranspiration at the Fringe of the Botswana Kalahari, with Emphasis on Deep Rooting Vegetation. PhD thesis.

O'Brien, J. J., Oberbauer, S. F., and Clark, D. B. (2004). Whole tree xylem sap flow responses to multiple environmental variables in a wet tropical forest. Plant, Cell \& Environment, 27(5):551-567.

Oltchev, A., Čermák, J., Gurtz, J., Tishenko, A., Kiely, G., Nadezhdina, N., Zappa, M., Lebedeva, N., Vitvar, T., Albertson, J. D., Tatarinov, F., Tishenko, D., Nadezhdin, V., Kozlov, B., Ibrom, A., Vygodskaya, N., and Gravenhorst, G. (2002a). The response of the water fluxes of the boreal forest region at the Volga's source area to climatic and land-use changes. Physics and Chemistry of the Earth, Parts $A / B / C$, 27(9-10):675-690.

Oltchev, A., Cermak, J., Nadezhdina, N., Tatarinov, F., Tishenko, A., Ibrom, A., and Gravenhorst, G. (2002b). Transpiration of a mixed forest stand: field measurements and simulation using svat models. Boreal environment research, 7(4):389-397.

Paço, T. A., David, T. S., Henriques, M. O., Pereira, J. S., Valente, F., Banza, J., Pereira, F. L., Pinto, C., and David, J. S. (2009). Evapotranspiration from a mediterranean evergreen oak savannah: The role of trees and pasture. Journal of Hydrology, 369(1-2):98-106.

Phillips, D. L. and Gregg, J. W. (2003). Source partitioning using stable isotopes: coping with too many sources. Oecologia, 136(2):261-269.

Porporato, A., Laio, F., Ridolfi, L., and Rodriguez-Iturbe, I. (2001). Plants in water-controlled ecosystems: active role in hydrologic processes and response to water stress: III. Vegetation water stress. Advances in Water Resources, 24(7):725-744. 
Poyatos, R., Cermak, J., and Llorens, P. (2007). Variation in the radial patterns of sap flux density in pubescent oak (quercus pubescens) and its implications for tree and stand transpiration measurements. Tree Physiol, 27(4):537-548.

Quinn, G. P. and Keough, M. J. (2002). Experimental Design and Data Analysis for Biologists. Cambridge University Press.

Regalado, C., Ritter, A., and Aschan, G. (2009). Tdp measurements to determine nighttime sap-flow and the impact of fog in tree transpiration of a laurisilva forest. Acta Hort. (ISHS), 846:367-374.

Regalado, C. M. and Ritter, A. (2007). An alternative method to estimate zero flow temperature differences for granier's thermal dissipation technique. Tree Physiol, 27(8):1093-1102.

Reilly, J. M. and Fuglie, K. O. (1998). Future yield growth in field crops: what evidence exists? Soil and Tillage Research, 47(3):275-290.

Reis, F. D., Campostrini, E., de Sousa, E. F., and Silva, M. G. E. (2006). Sap flow in papaya plants: Laboratory calibrations and relationships with gas exchanges under field conditions. Scientia Horticulturae, 110(3):254-259.

Reyes-Acosta, J. L. and Lubczynski, M. W. (2014). Optimization of dryseason sap flow measurements in an oak semi-arid open woodland in Spain. Ecohydrology, 7(2):258-277.

Reyes-Acosta, J. L., Vandegehuchte, M. W., Steppe, K., and Lubczynski, M. W. (2012). Novel, cyclic heat dissipation (chd) method for the correction of natural temperature gradients in sap flow measurements. part 2. laboratory assessment. Tree Physiology.

Rice, J. (2006). Mathematical statistics and data analysis. Thomson Learning.

Ritter, A., Muñoz Carpena, R., and Regalado, C. (2011). Capacidad de predicción de modelos aplicados a La Zona Saturada del Suelo: herramienta informática para la adecuada evaluación de la bondad-de-ajuste con significación estadística. In Fernández, J. and Martin, N.S., ., editors, Estudios en la zona no saturada del suelo : ZNS11, volume X, pages 259-264, Salamanca : Universidad de Salamanca.

Rodriguez-Iturbe, I., Porporato, A., Laio, F., and Ridolfi, L. (2001). Plants in water-controlled ecosystems: active role in hydrologic processes and response to water stress: I. Scope and general outline. Advances in Water Resources, 24(7):695-705.

Rothfuss, Y., Biron, P., Braud, I., Canale, L., Durand, J.-L., Gaudet, J.-P., Richard, P., Vauclin, M., and Bariac, T. (2010). Partitioning evapotranspiration fluxes into soil evaporation and plant transpiration using water stable isotopes under controlled conditions. Hydrological Processes, 24(22):3177-3194.

Sadava, D., Hillis, D., Heller, H., and Berenbaum, M. (2009). Life: The Science of Biology, volume 3. WH Freeman, (8th edition) edition. 
Sala, A. and Tenhunen, J. D. (1994). Site-specific water relations and stomatal response of Quercus ilex in a Mediterranean watershed. Tree Physiology, 14(6):601-617.

Saveyn, A., Steppe, K., and Lemeur, R. (2008). Spatial variability of xylem sap flow in mature beech (fagus sylvatica) and its diurnal dynamics in relation to microclimate. Botany-Botanique, 86(12):1440-1448.

Scanlon, B., Healy, R., and Cook, P. (2002). Choosing appropriate techniques for quantifying groundwater recharge. Hydrogeology Journal, 10(1):18-39. 10.1007/s10040-001-0176-2.

Schewe, J., Heinke, J., Gerten, D., Haddeland, I., Arnell, N. W., Clark, D. B., Dankers, R., Eisner, S., Fekete, B. M., Colón-González, F. J., et al. (2014). Multimodel assessment of water scarcity under climate change. Proceedings of the National Academy of Sciences, 111(9):3245-3250.

Scholz, F. C., Bucci, S. J., Goldstein, G., Meinzer, F. C., Franco, A. C., and Miralles-Wilhelm, F. (2008). Temporal dynamics of stem expansion and contraction in savanna trees: withdrawal and recharge of stored water. Tree Physiology, 28(3):469-480.

Schwinning, S., Davis, K., Richardson, L., and Ehleringer, J. R. (2002). Deuterium enriched irrigation indicates different forms of rain use in shrub/grass species of the Colorado Plateau. Oecologia, 130(3):345355.

Seckler, D. W. and International Water Management, I. (1998). World Water Demand and Supply, 1990 to 2025: Scenarios and Issues. International Water Management Institute, Colombo, Sri Lanka.

Sena Gomes, A. and Kozlowski, T. (1980). Effects of flooding on eucalyptus camaldulensis and eucalyptus globulus seedlings. Oecologia, 46(2):139-142.

Silva, J. S. and Rego, F. C. (2003). Root distribution of Mediterranean woody plants . Introducing a new empirical model. Plant biosystems, 137(June 2002):1-10.

Simonneau, T., Habib, R., Goutouly, J., and Huguet, J. (1993). Diurnal changes in stem diameter depend upon variations in water content: direct evidence in peach trees. Journal of Experimental Botany, 44(3):615621.

Smit, C., den Ouden, J., and Díaz, M. (2008). Facilitation of Quercus ilex recruitment by shrubs in Mediterranean open woodlands. Journal of Vegetation Science, 19(2):193-200.

Stehman, S. V. and Czaplewski, R. L. (1998). Design and analysis for thematic map accuracy assessment: Fundamental principles. Remote Sensing of Environment, 64(3):331-344.

Steppe, K., Cnudde, V., Girard, C., Lemeur, R., Cnudde, J. P., and Jacobs, P. (2004). Use of x-ray computed microtomography for non-invasive determination of wood anatomical characteristics. Journal of Structural Biology, 148(1):11-21. 
Steppe, K., De Pauw, D. J., and Lemeur, R. (2008). Validation of a dynamic stem diameter variation model and the resulting seasonal changes in calibrated parameter values. Ecological Modelling, 218(3):247-259.

Steppe, K., De Pauw, D. J. W., Doody, T. M., and Teskey, R. O. (2010). A comparison of sap flux density using thermal dissipation, heat pulse velocity and heat field deformation methods. Agricultural and Forest Meteorology, 150(7-8):1046-1056.

Steppe, K., De Pauw, D. J. W., Lemeur, R., and Vanrolleghem, P. A. (2006). A mathematical model linking tree sap flow dynamics to daily stem diameter fluctuations and radial stem growth. Tree Physiology, 26(3):257273.

Steppe, K. and Lemeur, R. (2004). An experimental system for analysis of the dynamic sap-flow characteristics in young trees: results of a beech tree. Functional Plant Biology, 31(1):83-92.

Steppe, K. and Lemeur, R. (2007a). Effects of ring-porous and diffuseporous stem wood anatomy on the hydraulic parameters used in a water flow and storage model. Tree Physiology, 27(1):43-52.

Steppe, K. and Lemeur, R. (2007b). Effects of ring-porous and diffuseporous stem wood anatomy on the hydraulic parameters used in a water flow and storage model. Tree Physiology, 27(1):43-52.

Steppe, K., Lemeur, R., and Dierick, D. (2005). Unravelling the relationship between stem temperature and air temperature to correct for errors in sap-flow calculations using stem heat balance sensors. Functional Plant Biology, 32(7):599-609.

Stöhr, A. and Lösch, R. (2004). Xylem sap flow and drought stress of fraxinus excelsior saplings. Tree Physiology, 24(2):169-180.

Story, M. and Congalton, R. (1986). Accuracy assessment: a user's perspective. Photogrammetric Engineering and remote sensing, 52(3):397399.

Swanson, R. H. (1994). Significant historical developments in thermal methods for measuring sap flow in trees. Agricultural and Forest Meteorology, 72(1-2):113-132.

Tang, J., Bolstad, P. V., Ewers, B. E., Desai, A. R., Davis, K. J., and Carey, E. V. (2006). Sap flux-upscaled canopy transpiration, stomatal conductance, and water use efficiency in an old growth forest in the great lakes region of the united states. J. Geophys. Res., 111(G2):G02009.

Tanvir Hassan, S., Lubczynski, M. W., Niswonger, R. G., and Su, Z. (2014). Surface-groundwater interactions in hard rocks in sardon catchment of western spain: an integrated modeling approach. Journal of Hydrology.

Tatarinov, F. A., Kucera, J., and Cienciala, E. (2005). The analysis of physical background of tree sap flow measurement based on thermal methods. Measurement Science and Technology, 16:1157-1169.

Terradas, J. and Savé, R. (1992). The influence of summer and winter stress and water relationships on the distribution of quercus ilex 1. Plant Ecology, 99-100:137-145. 10.1007/BF00118219. 
Thorburn, P. J., Hatton, T. J., and Walker, G. R. (1993). Combining measurements of transpiration and stable isotopes of water to determine groundwater discharge from forests. Journal of Hydrology, 150(24):563-587.

Tributsch, H., Nadezhdina, N., and Čermák, J. (2006). Infrared images of heat fields around a linear heater in tree trunks: what can be learned about sap flow measurement? Ann. For. Sci., 63(7):653-660.

Turner, J. V., Farrington, P., and Gailitis, V. (2001). Extraction and Analysis of Plant Water for Deuterium Isotope Measurement and Application to Field Experiments. Kluwer Academic, Dordrecht:.

Tyree, M. T. (1988). A dynamic model for water flow in a single tree: evidence that models must account for hydraulic architecture. Tree Physiology, 4(3):195-217.

Tyree, M. T., Snyderman, D. A., Wilmot, T. R., and Machado, J.-L. (1991). Water Relations and Hydraulic Architecture of a Tropical Tree (Schefflera morototoni) : Data, Models, and a Comparison with Two Temperate Species (Acer saccharum and Thuja occidentalis). Plant Physiology, 96(4):1105-1113.

Tyree, M. T. and Zimmerman, M. H. (2002). Xylem Structure and the Ascent of Sap. Springer series in wood science;. Springer, Berlin etc., (2nd edition) edition.

USGS (2012). The Water Cycle (Water Science for Schools.

Vandegehuchte, M. W. and Steppe, K. (2012). Sapflow+: a four-needle heat-pulse sap flow sensor enabling nonempirical sap flux density and water content measurements. New Phytologist, 196(1):306-317.

Verbeeck, H., Steppe, K., Nadezhdina, N., Op de Beeck, M., Deckmyn, G., Meiresonne, L., Lemeur, R., Cermak, J., Ceulemans, R., and Janssens, I. A. (2007). Stored water use and transpiration in scots pine: a modeling analysis with anafore. Tree Physiology, 27(12):1671-1685.

Vertessy, R. A., Hatton, T. J., Reece, P., O’Sullivan, S. K., and Benyon, R. G. (1997). Estimating stand water use of large mountain ash trees and validation of the sap flow measurement technique. 17:747-756.

Vrugt, J., Gupta, H., Bastidas, L., Bouten, W., and Sorooshian, S. (2003a). Effective and efficient algorithm for multiobjective optimization of hydrologic models. Water Resour. Res, 39(8):1214.

Vrugt, J. and Robinson, B. (2007). Improved evolutionary optimization from genetically adaptive multimethod search. Proceedings of the National Academy of Sciences, 104(3):708.

Vrugt, J., Ter Braak, C., Diks, C., Robinson, B., Hyman, J., and Higdon, D. (2009). Accelerating markov chain monte carlo simulation by differential evolution with self-adaptive randomized subspace sampling. International Journal of Nonlinear Sciences and Numerical Simulation, 10(3):273-290. 
Vrugt, J. A., Gupta, H. V., Bouten, W., and Sorooshian, S. (2003b). A shuffled complex evolution metropolis algorithm for optimization and uncertainty assessment of hydrologic model parameters. Water Resources Research., 39(8):1201.

Vrugt, J. A., Hyman, J. M., Robinson, B. A., Higdon, D., Ter Braak, C. J. F., and Diks, C. G. H. (2008). Accelerating Markov Chain Monte Carlo simulation by differential evolution with self-adaptive randomized subspace sampling. Journal Name: International Journal of Nonlinear Sciences and Numerical Simulation, page Medium: ED. [Los Alamos National Laboratory] [Netherlands] [Univ. of Amsaterdam].

Walker, C. D. and Richardson, S. B. (1991). The use of stable isotopes of water in characterising the source of water in vegetation. Chemical Geology : Isotope Geoscience Section, 94(2):145-158.

Wershaw, R. L., Friedman, I., Heller, S. J., and Frank, P. A. (1966). Hydrogen Isotope Fractionation of Water Passing through Trees. Pergamon, New York.

Wheeler, T. D. and Stroock, A. D. (2008). The transpiration of water at negative pressures in a synthetic tree. Nature, 455(7210):208-212.

White, J. (1989). Stable hydrogen isotope ratios in plants: a review of current theory and some potential applications, volume 68. SpringerVerlag, Heidelberg.

Whitley, R., Medlyn, B., Zeppel, M., Macinnis-Ng, C., and Eamus, D. (2009). Comparing the penman-monteith equation and a modified jarvisstewart model with an artificial neural network to estimate stand-scale transpiration and canopy conductance. Journal of Hydrology, 373(12):256-266.

Williams, D. G., Cable, W., Hultine, K., Hoedjes, J. C. B., Yepez, E. A., Simonneaux, V., Er-Raki, S., Boulet, G., de Bruin, H. A. R., Chehbouni, A., Hartogensis, O. K., and Timouk, F. (2004). Evapotranspiration components determined by stable isotope, sap flow and eddy covariance techniques. Agricultural and Forest Meteorology, 125(3-4):241-258.

Williams, M., Bond, B., and Ryan, M. (2001). Evaluating different soil and plant hydraulic constraints on tree function using a model and sap flow data from ponderosa pine. Plant, Cell \& Environment, 24(7):679-690.

Williams, M., Rastetter, E., Fernandes, D., Goulden, M., Wofsy, S., Shaver, G., Melillo, J., Munger, J., Fan, S., and Nadelhoffer, K. (1996). Modelling the soil-plant-atmosphere continuum in a Quercus-Acer stand at Harvard Forest: the regulation of stomatal conductance by light, nitrogen and soil/plant hydraulic properties. Plant, Cell and Environment, 19(8):911-927.

Wilson, K. B., Hanson, P. J., Mulholland, P. J., Baldocchi, D. D., and Wullschleger, S. D. (2001). A comparison of methods for determining forest evapotranspiration and its components: sap-flow, soil water budget, eddy covariance and catchment water balance. Agricultural and Forest Meteorology, 106(2):153-168. doi: DOI: 10.1016/S01681923(00)00199-4. 
Wullschleger, S. D., Hanson, P. J., and Todd, D. E. (2001). Transpiration from a multi-species deciduous forest as estimated by xylem sap flow techniques. Forest Ecology and Management, 143(1-3):205-213. doi: DOI: 10.1016/S0378-1127(00)00518-1.

Wullschleger, S. D., Meinzer, F. C., and Vertessy, R. A. (1998). A review of whole-plant water use studies in trees. 18:499-512.

Yaseef, N. R., Yakir, D., Rotenberg, E., Schiller, G., and Cohen, S. (2010). Ecohydrology of a semi-arid forest: partitioning among water balance components and its implications for predicted precipitation changes. Ecohydrology, 3(2):143-154.

Zencich, S., Froend, R., Turner, J., and Gailitis, V. (2002). Influence of groundwater depth on the seasonal sources of water accessed by Banksia tree species on a shallow, sandy coastal aquifer. Oecologia, 131(1):8-19.

Zeppel, M. J. B., Murray, B. R., Barton, C., and Eamus, D. (2004). Seasonal responses of xylem sap velocity to vpd and solar radiation during drought in a stand of native trees in temperate australia. Functional Plant Biology, 31(5):461-470.

Zweifel, R. and Häsler, R. (2001). Dynamics of water storage in mature subalpine picea abies: temporal and spatial patterns of change in stem radius. Tree Physiology, 21(9):561-569.

Zweifel, R., Item, H., and Häsler, R. (2000). Stem radius changes and their relation to stored water in stems of young Norway spruce trees. Trees-Structure and Function, 15(1):50-57.

Zweifel, R., Item, H., and Häsler, R. (2001). Link between diurnal stem radius changes and tree water relations. Tree Physiology, 21(12-13):869877. 


\section{ITC dissertations}

A complete list of ITC dissertations is online on the ITC website: www.itc.nl/research/phd/phd_graduates.aspx.

This dissertation has number 272 . 